

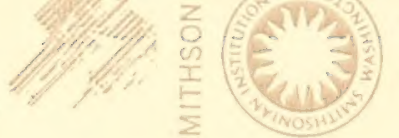

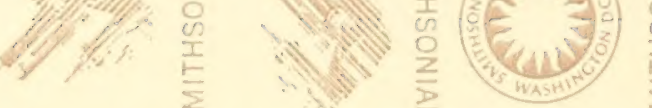

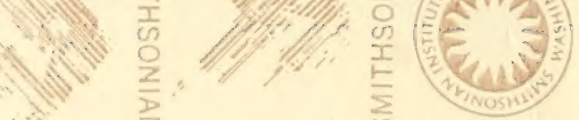

S. Nat)

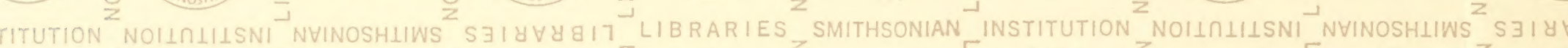

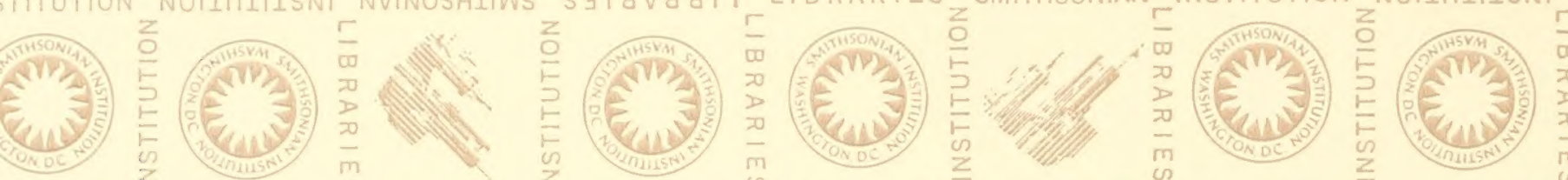

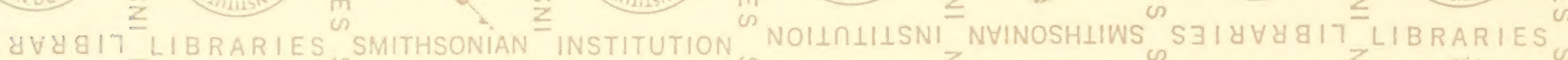

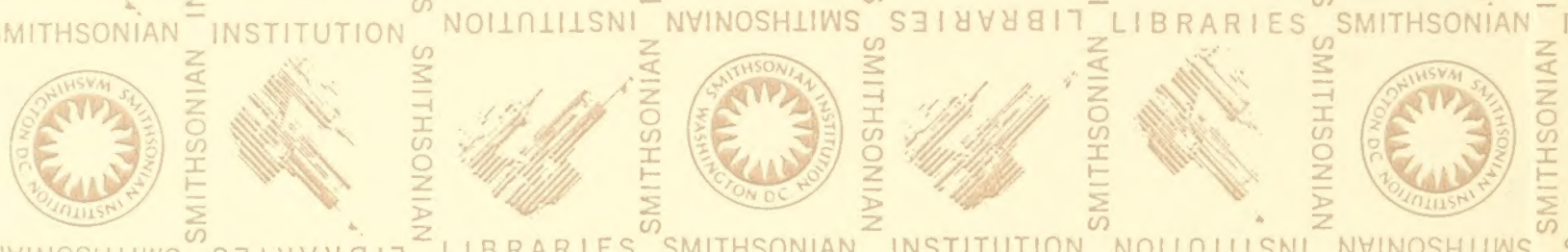

(3) 10.10

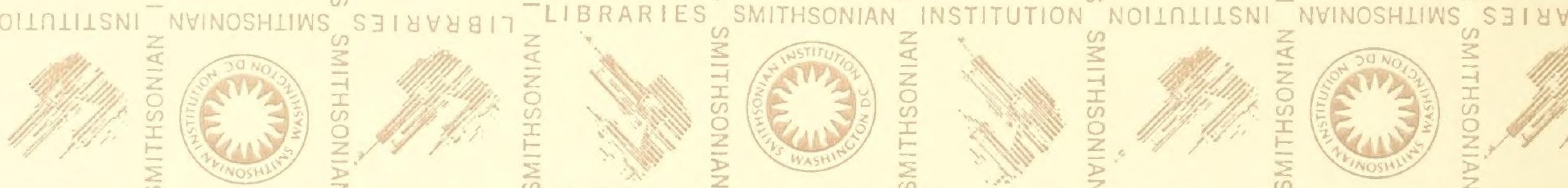
Navan (2)

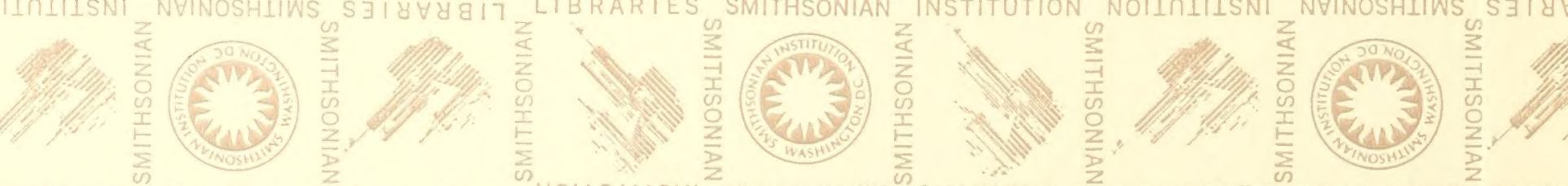




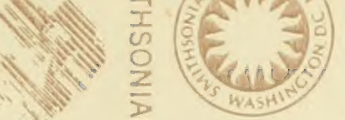

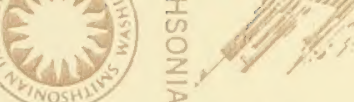

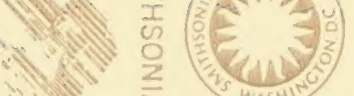

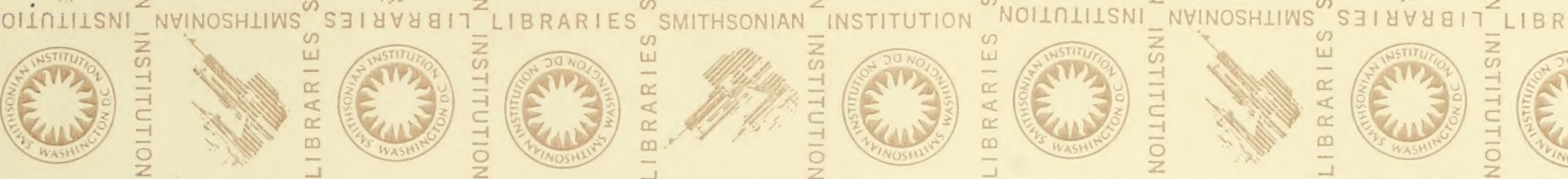

IBRARIES SMITHSONIAN INSTITUTION NOILNLIISNI NVINOSHLIWS S I Y VY GI7 LIBRARIES SMITHSONIAN INSTITUTIO

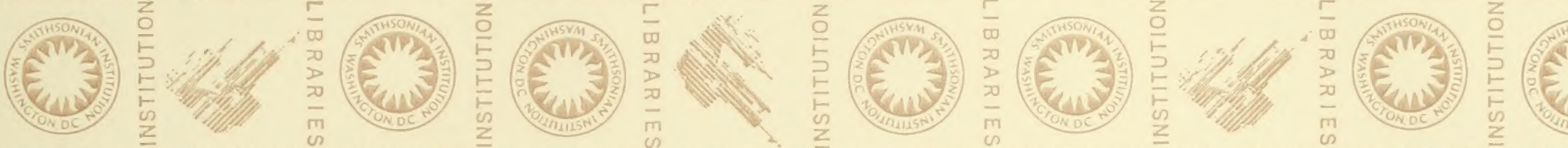

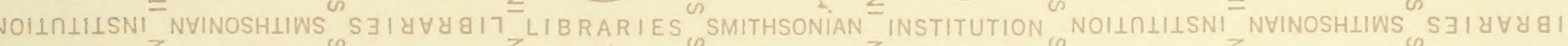

1).

IBRARIES SMITHSONIAN INSTITUTION NOIINLIISNI NVINOSHIIW

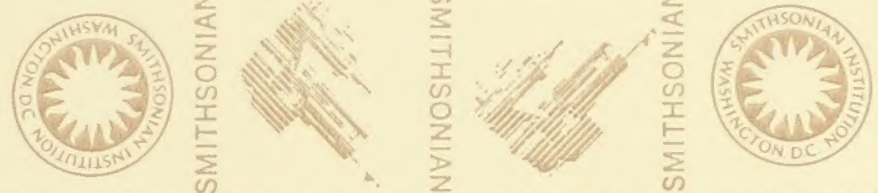

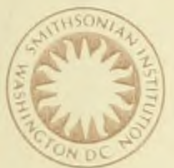

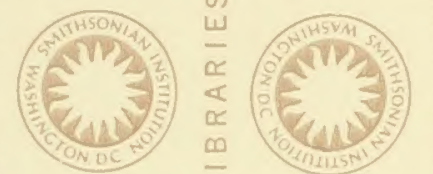

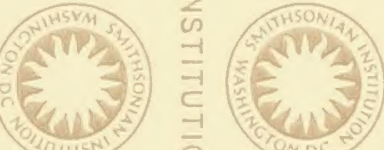

$\left[\frac{212}{2}\right.$

$1011 M 1 I \perp$ SNI NHINOSHLIWS $^{2}$

(N)

Nivis

(2) (2)

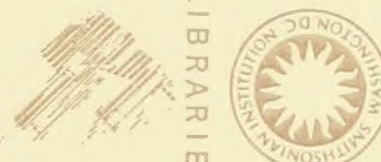

(3)

(

IBRARIES SMITHSONIAN

MSTITUTION NOIINLIISNI NVINOSHIIWS SJI YVYG

$+3$

(2)

(1)

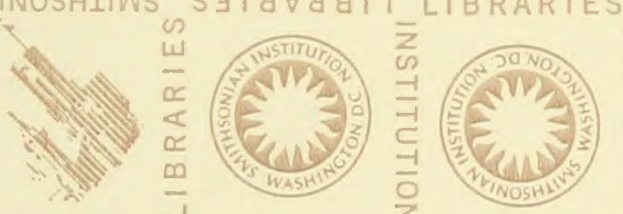

s)

(2)

(3)

(n)

(2)

Ist

(

留

(

(3)

(1)

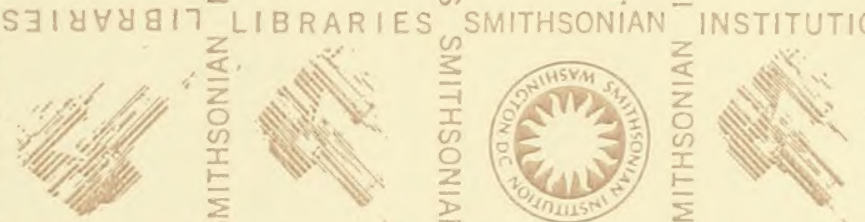

24

(3)

\section{B}

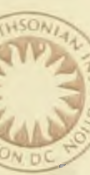

(a)

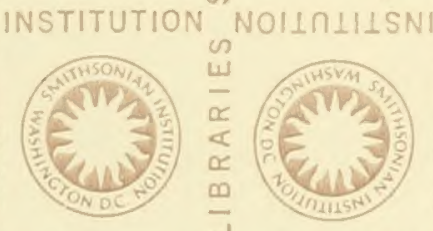

in

(1)

(i)

(3)

行

(3)

N

( )

结要

(

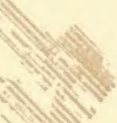

$\sum^{2}(210)$
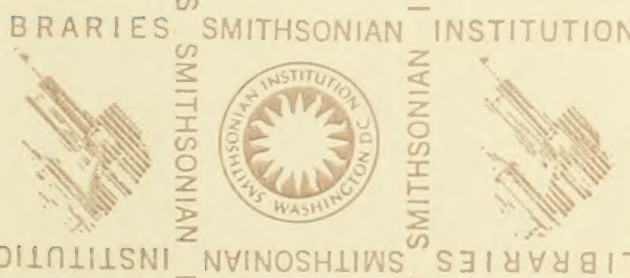

Ha
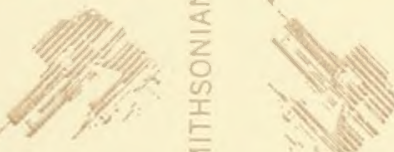

(n) 


Livr. $X$.

\section{Siboga-Expeditie}

ZOOLOGIQUUES, BOTANIQUUS, OCÉANOGRAPHIQUES ET GÉOLOGIQUES

ENTREPRISES AUX

INDES NÉERLANDAYSES ORIGNTALES En 1899-1900,

à bord du SIBOGA

\section{SOUS LE COMMANDEMENT DE}

G. F. TYDEMAN

PUBLTÉS PAR

M A X W H H H R

Chẹ de l'expédition.

* I. Intraduction et description de l'expédition, Max Weber *II. Le batea et son équipement scientilique, G. F. T y de man III. Résultats hydrographiques, G. F. Ty de man.

IV. Foraminifera.

V. Radiolaria, M. Hartmann.

* VI. Porifera, G. C. J. Vosmaer et J. H. Vernhoul ').

VII. Hydropolypi, Ch. Julin.

VIII. Hydrocorallinae, S. J. Hick on.

IX. Siphonophora, Mlles Leas et van Riemsdijk.

X. Hydromedusae, O. Mas.

* XI. Scyphomedusae, O Ma as.

XII. Ctenophora, Mlle F. Moser.

* XIII. Gorgonidae, Aleyonidae, J. Verslu y s ${ }^{1}$ ).

XIV. Pennatulidae, S. J. H i ckson.

XV. Actiniaria, P. Me Murrich.

*XVI. Madreporaxia, A. A le oc k ${ }^{2}$ ) et L. Döderlein.

XVII. Antipatharia, P. N. va a Ka m pen.

XVIIL. Turbellaria, L, von Graff et R. R. von Stummer.

XIX. Cestodes, J. W. Speugel.

XX. Nematodes, A. A. W. Hubrech t

XXI. Chretognatha, G. H. Fow ler.

XXII. Nemertini, A. A. W. Hubrecht.

XXUI. Myzostomidae, R. R, von Stummer.

XXIVa. Polychaeta errantia, R. Horst.

XXIVi. Polychaeta sedentaria, M. Caullery et F. Mesnil.

* XXV. Gephyrea, C. Ph. Sluiter.

XXVI. Enteropueusta, J. W. Spengel.

XXVII. Brachiopodn, J. F, $\vee$ a u Bemmelen.

XXVIII. Bryozoa, S. F. Harmer.

XXIX. Copepoda, A. Scott

XXX. Ostracoda, G. W. M üller.

XXXI. Cirrhipedia, P. P. C. Hoek.

XXXIIa. Isopoda, H. J. Ha n se n.

XXXIIb. Epicaridae, J. Bonn ier.

XXX111. Amphipoda, J. Bonnier.

XXXIV. Caprellidae, P. Ma yer.

XXXV. Stomatopoda, H. J. Hansen.

XXXVI. Leptostraca, H. J. Hansen.

XXXVII. Schizopoda, H. J. Hansen.

XXXVIII. Sergestidae, H. J. H an sen.

XXXIX. Decapoda, J. G. d e Mau.

XL. Pantopoda; J. C. C. Loman.

XLI. Halobatidae, J. Th. Ou demans.

XLII. Crinoidea, I. Döderle in p p.

XLIII. Echinoidea, J. C. H. de Meyere.

* XLIV. Holothurioidea, C. Ph. Sl uiter.

XLV. Ophiuroidea, R. Köhler.

XIVI. Asteroidea, L. Döderlein.

* XLVII. Solenogastres, H. F. N ierst $r$ asz.

XLVIII. Chitonidae, H. F. Nierstrasz.

XLIX. Prosobranchia, M. M. Schepman.

L. Opisthobranchia, $R$. Bergh.

LI. Heteropoda, J. J. Te sch.

LII. Pteropoda, J, J, Tesch.

LIII. Lamellibranchiata. P. Pel seneer et Ph, Dautzenberg.

LIV. Scaphopods.

LV. Cephalopoda, L, Joubin.

LVI. Tunicata, C. Ph. Sluiter.

LVII. Pisces, Max Weber.

LVIII. Cetacea, Max Weber

LIX. Liste des algues, Mme A. We ber.

* LX. Halimeda, Mlle E. S. Bartan. (Mme E. S. Gepp).

LXI. Melobesiere, Mmo A. Weber et M. Foslie.

LXII. Dinoflagellati. Coccosphaeridae, J. P. T,otsy.

LXIII. Diatomaceae, J. P. Lotsy.

LXIV. Deposita mariną, O. B. Bögg ild

LXV. Résultats géologiques, A. Wi chmann.

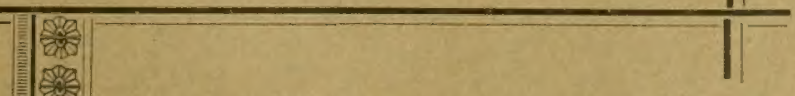

DIE

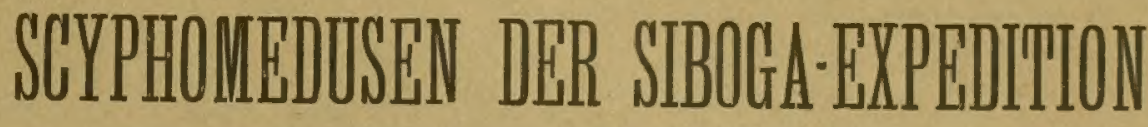

VON

\section{OTTO MAAS}

Professor der Zoologie an der Universität München

Mit XII Tafeln

Monographie XI aus:

\section{UITKOMSTEN OP ZOOLOGISCH, BOTANISCH, OCEANOGRAPHISCH EN GEOLOGISCH GEBIED}

verzameld in Nederlandsch Oost-Indië I899-1900

aan boord H. M. Siboga onder commando van

Luitenant ter zee Ie kl. G. F. TYDEMAN

UITGEGEVEN DOOR

Dr. MAX WEBER

Prof. in Amsterdam, Leider der Expedıtie

(met medewerking van de Maatschappij ter bevordering van het. Natuurkundig onderzoek der Nederlandsche Koloniën)

BOEKHANDEL EN DRUKKERIJ

E. J. BRILL LEIDEN 


\section{Voor de uitgave van de resultaten der Siboga-Expeditie hebben}

\section{bijdragen beschikbaar gesteld:}

De Maatschappij ter bevordering van het Natuurkundig Onderzoek der Nederlandsche Koloniën.

Het Ministerie van Koloniën.

Het Ministerie van Binnenlandsche Zaken.

Het Koninklijk Zoologisch Genootschap "Natura Artis Magistra" te Amsterdam.

De „Oostersche Handel en Reederij" te Amsterdam.

De Heer B. H. DE WAAL Oud-Consul-Generaal der Nederlanden te Kaapstad. 
SIBOGA-EXPEDITIE. 


\section{Siboga-Expeditie}

\section{UITKOMSTEN}

OP

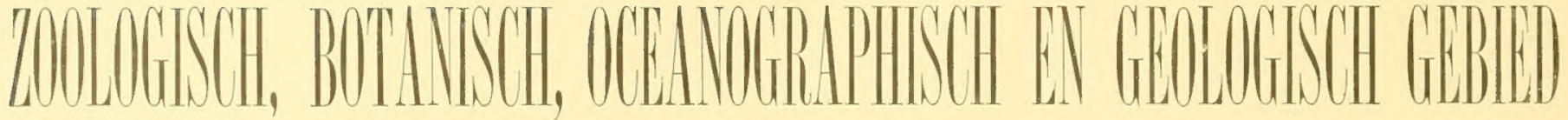

VERZAMELD IN

NEDERLANDSCH OOST-INDIË I 899-i900

A AN BOOR.D H. M. SIBOGA ONDER COMMANDO VAN Luitenant ter zee $1^{c}$ kl. G. F. TYDEMAN

UITGEGEVEN DOOR

Dr. MAX WEBER

Prof. in Amsterdam, Leider der Expeditie

(met medewerking van de Maatschappij ter bevordering van het Natuurkundig onderzoek der Nederlandsche Koloniën)

BOEKHANDEL EN DRUKKERIJ

E. J. BRILL 


\title{
DIE SCYPHOMEDUSEN DER SIBOGA-EXPEDITION
}

\section{DR. O'TTO MAAS \\ Professor der Zoologie an der Universität Müuchen.}

\author{
Mit XII Tafeln
}





\title{
DIE SCYPHOMEDUSEN DER SIBOGA-EXPEDITION
}

VON

\author{
Dr. OTTO MAAS
}

Docent der Zoologie an der Universität München.

Mit NII Tafeln

\section{Vorwort.}

Trotzdem die Arbeiten der Siboga-Expedition in erster Linie dem tiefen Meeresboden galten, wurde doch auch das Sammeln pelagischen Materials mit ausgezeichnetem Erfolg betrieben. Die Medusen, craspedote sowohl wie acraspede, bilden eine nach Exemplaren wie nach Artenzahl sehr stattliche Sammlung, welche Vertreter fast aller Gruppen und Untergruppen enthält. Die Acraspeden oder Scyphomedusen der Expedition zählen I 9 Gattungen und 2 I Arten; darunter sind 2 neue Gattungen, 4 neue Arten und mehrere neue Varietäten, ferner mehrere Arten, die bisher verschollen oder selten waren, und endlich solche; die erst von der gerade vorhergehenden deutschen Tiefsee-Expedition zum ersten Mal bekannt geworden sind. Nach dem System geordnet, sind die Arten folgende:

I. Charybdeïla.

I. Tamoja bursaria Hckl.

II. Coronata.

2. Pericolpa campana Hckl.

3. Periphylla dodecabostrycha Brdt.

4. Atorella subglobosa Vanh.

5. Atolla Valdiviae Vanh.

6. Paraphyllina intermadia nov. gen. n. sp.

7. Nausithoe picta A. Ag. u. Mayer.

S. Linerges draco Hck1. 
III. Discophora.

A. Semaeostomea.

9. Aurclia aurita L. var. colpota Brdt.

Io. Cyanca ferrugina Eschsch.

I I. Pelagia panopyra Pér. \& Les.

B. Rhizostomea.

I2. Netrostoma cocmulescens n. sp.

13. Cassiopeja andromeda Eschsch. nov. var. malayensis.

I4. Cassiopicia ornata Hckl. nov. var. digitata.

15. Crambione mastigophora nov. gen. n. sp.

16. Crossostoma anadyomene n. sp.

I7. Mastigias papua L. Ag. nov. var. Sibogac.

I8. Mastigias occellata Modeer.

I9. Rhopilcma hispidum Vanh.

20. Thy'sanostoma thy'sanura Hckl.

2i. Himantostoma flagellatum Hckl.

Mehrere dieser Arten sind von allgemeinerer Bedeutung, und fiur die Morphologie der ganzen Medusengruppe verwertbar. Es sind das in erster Linie Formen aus grösserer Tiefe, so Pericolpa campana, die erste Pericolpide, oder wirklich 4 zählige, coronate Meduse, die seit HAECKEL'S Beschreibung wieder aufgefunden worden ist, ferner die interessante 6 zählige Atorclla subglobosa, die bisher nur in einem einzigen Exemplar von der deutschen Tiefsee-Expedition durch VAxiöffen bekannt war. Ausserdem sind die Genera Periphylla und Atolla selbst vertreten, letzteres in einer von der Valdivia neuen Art in zahlreichen Exemplaren verschiedenen Alters. Vor allem ist jedoch die neue Gattung Paraphyllina zu nennen, die durch die 4-Zahl der Rhopalien und r2-Zahl der Tentakel Poriphylla gleicht, jedoch nach einem ganz andern Typus gebaut ist und dadurch für die Systematik sehr wichtig wird. Nausithoe picta ist wegen der Gonadenstellung von Interesse, und in Lincrges draco konnte seit HaEcrel zum ersten Mal ein Vertreter dieser abweichenden Familie, von der noch manche Organisationsverhältnisse unbekannt sind, beschrieben werden, und zwar nach gut conserviertem Material. Unter den Semaeostomen ist Cyanea formginca zu nennen, die seit Eschischoltz nur noch von Goette aufgeführt wurde; die Arten von Aurclia und Pclagia kommen fur die Fragen der geographischen $V$ erbreitung in Betracht. Besonders reich aber ist, wie sich in diesem wärmeren Meer erwarten liess, das Material an Rhizostomeen; es sind hier sowohl neue wie verschollene Gattungen grefunden worden, von anderen Arten konnten abweichende Varietäten constatiert worden; in mehreren Fallen wurden sehr zahlreiche Exemplare und ganze Entwicklungsserien fast von der Ephyra an bis zum erwachsenen Tier gefischt. Es sind Vertreter aller Unterabteilungen dieser grossen Acalephengruppe darunter, und es war dacturch möglich, eine eingehende Revision des so vielfach erörterten Systems der Rhizostomeen vorzunehmen.

Die Erhaltung war bei den meisten Exemplaren soweit sie nicht beim Aufziehen aus 
grosser Tiefe beschädigt wurden, eine sehr befriedigende, besonders durch $2-4 \%$ Formol; auch andere Flüssigkeiten, Chromgemische etc. haben gutes Dienste geleistet, wenn die Tiere nachher in Alkohol übergeführt, und nicht noch nachträglich in Formol gebracht wurden. Leider fehlen - was bei der grossen Arbeit an Bord nicht zu verargen ist -, Farbenskizzen der lebenden Tiere. Ich bin daher für Anwendung von Farben auf dasjenige angewiesen, was am Formolmaterial noch sichtbar ist, sowie auf besondere Notizen von anderer Seite. Um so mehr habe ich mich bemüht, den Habitus in Armform und Canalsystem wiederzugeben, und hierzu auch die Photographie herangezogen. Für die wohlgelungenen photographischen Aufnahmen habe ich Herrn Conservator Dr. Doflein meinen besonderen Dank abzustatten; ferner danke ich Herrn Prof. Chun und dem Verlag von Fischer, die es mir ermöglichten, in die Druckbogen der VANHöffes'schen Valdivia-Medusen Einsicht zu nehmen, wodurch mir und den Fachgenossen manche doppelte Beschreibung erspart blieb.

Nachfolgend gehe ich, ohne auf die grossen Züge des Systems zurückzukommen, direkt zur Beschreibung der gefundenen Gattungen und Arten uiber; die allgemeineren systematischen Beziehungen werden an einzelner Stelle angeschlossen und erörtert. Die Frage der verticalen Verbreitung ist bei den Tiefseecoronaten besprochen, (p. I 8), die geographische resp. horizontale Verbreitung soll zugleich mit der der craspedoten Medusen, die hierfür ein grösseres Material bieten, Erledigung finden.

M ï nchen. Zoolog. Institut.

August 1902. 


\title{
Beschreibung der gefangenen Arten.
}

\author{
I. Charybdeida.
}

\section{Tamoya.}

I. Tamoy'a bursaria Haeckel.

Tanoya bursaria Haeckel IS79.

Bursarius cythereae Lesson I 829 .

Charybdea grandis? A. Agass. \& Nayer 1902.

Stat. I70. I Exemplar von etwa $15 \mathrm{~cm}$. Glockenhöhe.

Diese auffallend grosse Charybdeide wurde in einem Verticalnetzfang aus 924 Meter Tiefe aufgezogen; ihr Erhaltungszustand erlaubt jedoch keine Beschreibung, sondern gerade noch eine Bestimmung. Habitus, Tentakel, deren Gallertsockel und das eigentümliche Velarium bezeichnen die Form sicher als Charybdeide. Was sich sonst noch erkennen lässt, insbesondere die Aufhängeart des Magens am Glockengrund und die Stellung der ansehnlichen FilamentGruppen sprechen für Tamoy'a im Gegensatz zur Gattung Charybdca selbst. Von dieser durch Fritz Mülter aufgestellten Gattung fiihrt Haeckel 2 pazifische Vertreter auf; beide nur nach der sehr unsicheren Darstellung Lessox's. Die eine "gargantuc" wohl kaum wiedererkenntlich, die andere bursaria hat friher eine besondere Gattung Bursarius gebildet, die aber von HaEckEL eingezogen wird, weil in ihrer Diagnose fälschlich nur 2 anstatt 4 Tentakel angegeben waren. Zur vorliegenden Gattung und Art scheint mir auch die A. Agassiz und Mayer'sche Charabdoa grandis zu zählen. Deren Abbildung (1902, pl. VII) spricht für mesenteriale Aufhängung des Magens und ebenso fur verticale Stellung der Filament-Gruppen. Auch die bemerkenswerte Grösse und sonstige Details unterstützen diese Vermutung. Im Text fehlt wie bei Mayer stets, jede Hervorhebung der Merkmale, durch die seine "neue" Art sich von den bisherigen unterscheiden würde. Die Gallerte der vorliegenden Form scheint sehr dick und zäh; das Exemplar war aber offenbar schon beim Aufziehen mechanisch geschädigt und zerbrochen. Unter den beschriebenen Charybdea-Arten, die übrigens (vgl. meine Bemerkungen is97 p. 85) kaum alle zu Recht bestehen, sondern zu vereinfachen sind, ist keine, die sich hierher beziehen liesse, da sie alle (auch rastoni Haeckel, plitippina Semper und arborifora Maas) wagrechte Filamentbüschel und eine freie Magenbasis ohne Mesenterien besitzen. 
II. Coronata.

Periphyllidae Haeckel is 79.

Trotz einiger alter Beschreibungen und Abbildungen ist die eigentümliche Gruppe der Periphylliden doch erst durch die neueren Expeditionen bekannt geworden. Sie wurde von HAECKEL nach Material des Challenger genauer umschrieben; dabei wurde ihr aber in Zusammenhang mit seiner schematisierenden Einteilung der Acraspeden eine völlige Sonderstellung zugesprochen, und eine eigene Ordnung der Peromedusen, gleichwertig mit Stauro-, Cubo-, und Discomedusen geschaffen (1879). Es ist das Verdienst Vanhöffen's (1892), die Systematik hier natürlicher gestaltet zu haben, indem er die nähere Verwandtschaft von Periphylla zu Nausithoe und somit auch zu Atolla erkannte. Durch die Vereinigung aller dieser Formen zur neuen Gruppe der "Coronata" wurden die Discomedusen von den heterogenen Cannostomen entlastet und bilden, nunmehr nur aus Semaeostomen und Rhizostomen bestehend, eine einheitliche und natürliche Gruppe. Diese Gruppe soll, wie VANHöffen weiterhin vorschlägt, als Acathammata, den Coronata plus sämmtlichen übrigen Acraspeden, die als Incoronata zusammengrefasst werden, also den Cathammata gegenüber stehen. In letztere Hinsicht konnte ich VANHöfFEN nicht folgen, sondern suchte die Incoronata, schon wegen der ganz eigenen Stellung der Charybdeiden, in ihre verschiedenartigen Bestandteile aufzulösen (I897), dagegen habe ich die Gruppe der Coronata durchaus acceptirt.

Die Vanhöffev'schen Darstellung der Periphylla und ihrer Verwandten war nach der grundlegenden HAECKEL'schen ein Fortschritt, ging aber von einem kleinen und teilweise zu schonenden Material aus. Ich konnte sie daher an den Medusen des Albatross in einer Reihe von Punkten modificieren und ergänzen, kam jedoch ebenfalls durch die Untersuchung der Organisation vieler Einzelheiten, besonders der Sinneskolben und Gonaden dazu, mich gegen den HAECKEL'schen Vergleich mit Charybdea und gegen die Sonderstellung, aber für die VANHöFfEx'sche Annäherung an Nausithoe anzusprechen (1897, p. 6r und ff.). Neuerdings hat VANiöffex auf Grund des schönen Materials der Valdivia-Expedition meine eingehende Darstellung in fast allen Teilen bestätigt, ausgenommen im Bau der Sinneskolben, wo er die von mir beschriebenen Ocellen durchaus in Abrede stellt (1902, p. 25). Sonst ist auch bei den Sinneskolben seine Beschreibung in Bezug auf Rhopalarcanal, dessen direkte Fortsetzung ohne Abschnürung bis zu den Concrementzellen, auf Sinnespolster, Deckschuppe mit der meinigen übereinstimmend.

Was nun die "Ocellen" betrifft, so habe ich s. Zt. ausdrücklich hervorgehoben, dass dieselben nichts mit den ectodermalen Augen von Charybdca zu thun haben, sondern nur das entodermale Pigment des Rhopalarcanals zeigen, sowie dass in ihrem Ectoderm keine linsenartige Differenzierung zu erkennen ist. $\mathrm{Zu}$ meiner Deutung als Ocellen bestimmte mich die besondere Höhe und Ausprägung der Entodermzellen, der Vergleich mit dem Becherauge von Aurclic, wo laut Schewikioff, ebenfalls die Pigmentschicht vom Entoderm geliefert wird (i $S 89$, p. 50), und endlich die Abfaltung vom ventralen Bulbus, die sie als besondere Gebilde erscheinen liess. VANHÖFFEN stellt nur einen einheitlichen Bulbus dar, und ebenso zeigt mir hier vorliegendes Material, dass diese Abfaltung nur durch den Erhaltungszustand bedingt war. Dennoch erscheint 
mir meine frihere Auslegung auch heute noch haltbar. Bei der vorliegenden Pcriphylla-Art, $P$. dodccabostrycha, ist, wie auch VAxiöFfEx angiebt, das charakteristische Pigment des Tieres, "dic 'Tiefscefärbung", durchaus auf den Centralmagen und das Mundrohr beschränkt; die peripheren Entodermteile, Lappentaschen, etc. sind gänzlich farblos, sodass die Gonaden durch die glashelle Gallerte durchschimmern. Um so auffälliger ist das plötzlich im Bulbus des Rhopaliums angehäufte rotbraune Pigment. Der Ventralbulbus greift, wie auch VAxröffen beschreibt, seitlich heriber, sodass nur die dorsale Nittellinie des Rhopaliums davon frei bleibt. Gerade diese seitlich hervortretenden Teile zeigen das Pigment besonders ausgesprochen, und in ihnen ist auch das Ectoderm stark erhöht und etwas eingesenkt (Taf. II, Fig. I 5 ect!). Natürlich ist eine solche Bildung weder mit den Augen der Charybda noch mit dem ventralen Becherauge der Nausithoc, das ectodermales P'igment und linsenartige Zellen besitzt, zu vergleichen. Dies fehlt hier vollkommen, trotz der sonstigen Aehnlichkeit des Kolbens mit dem von Nausithoe, findet sich aber wieder bei einer andern, ganz neuen, zwischen Periphylla und Nausithoc stehenden Gattung (s. u. p. 7 u. 9). Aber doch kann man bei den hier-vorliegenden Gebilden auch im Anschluss an Screwiaroff's Ausführungen und Abbildungen, an primitive Organe der Lichtperception denken. Die gestreckte Gesammtform des Kolbens, die ich schon früher als tentakelähnlich beschrieben, im Gegensatz zu dem gedrungenen Rhopalium der Nausithoe, tritt auch hier hervor, ebenso die Kürze und Kleinheit der Deckschuppe (Fig. I 5 sq).

Bezüglich der ïbrigen Organisation der Periphylliden kann ich wie VAxHöFfEN auf meine frihere Darstellung verweisen. Auch habe ich daselbst die Systematik der Gruppe etwas zu vereinfachen gesucht. Ton den HAEcrel'schen 4 Genera der Peromedusen habe ich ausser Periphylla selbst nur noch eine aufrecht erhalten, in der Gattung Pcriphylla selbst $P$. mirabitis eingezogen und die Unterschiede zwischen $P$. dodecabostrycha und $P$. regina präcisirt. VANHöfFEx hat zu diesen Merkmalen auch noch die Abgrenzung der $P$. hyacinthina gefügt und die I'Ewres'sche I'. humilis gestrichen. Somit werden jetzt nur diese 3 Arten unterschieden, die nebeneinander ohne locale Abgrenzung rorkommen. Von der Siboga wurde nur eine Art erbeutet.

\section{Periphylla Steenstrup.}

1. Periphylla dodecabostrycha Brandt i838. (Taf. II, Fig. I5. Taf. XII, Fig. Io7).

Stat. 230. Verticalnetz aus 2000 M. I Excmplar von 22 mm. Höhe, davon ro mm. Centralscheibe, I 2 mm. Lappenkranz, $16 \mathrm{~mm}$. Durchmesser. 2 Exemplare von etwa $12 \mathrm{~mm}$. Durchmesser.

P'ARAPHYLLinida: nov. fam.

Paraphyllina nov. gen.

Coronate Meduse mit 2 Tentakeln und 4 Rhopalien $z$ wischen 16 Randlappen. Die Rhopalien stehen perradial entsprechend den Magenecken, die $4 \times 3$ Tentakel inter-resp. adradial, entsprechend den Magenflächen uncl Filamenten. Mit $S$ eiförmigen Gonaden, dic parweise inter- 
radial liegen. Die Rhopalien zeigen ausser dem Otolithensack und dem ventralen Bulbus an letzterem ein ectodermales Auge mit Krystallinse und ectodermalem Pigment.

Der allgemeine Habitus der Meduse erinnert durch den flachgewölbten Schirm, das kurze Magenrohr, die Farblosigkeit der Umbrella etc. mehr an Nausithoe; dennoch würde man die Form wegen der charakteristischen Einteilung des Schirmrandes durch ${ }_{2} 2$ Tentakel und 4 Rhopalien zu Peraplylla rechnen, wenn nicht genauere Untersuchung ergäbe, dass diese Randgebilde zu den Hauptradien des Körpers gerade umgekehrt stehen, wie bei Periphylla. Da es sich hierbei nicht blos um die Lage der Gonaden handelt, die bei Coronaten (s. u. p. 2 I) bald perradial bald interradial genähert sein können, sondern wirklich um zwei verschiedene, "Baupläne", die allermindestens hier zur Aufstellung eines neuen Genus berechtigen, so ist zuvor eine genauere Prufung auch der friheren Formen in dieser Hinsicht vorzunehmen. Es ist zwar kaum anzu. nehmen, dass sich die früheren Beobachter, HAECKEL, VANHöffex, MAAs so übereinstimmend geirrt und nicht nur ein, sondern mehrere Organsysteme in falschen Radien liegend gezeichnet hätten; da aber doch bei fruheren Untersuchungen öfters nur sehr schlecht erhaltene Exemplare, besonders mit zerfetztem Magen vorlagen, da ferner die Stellung der Gonadenschenkel zu den Rhopalien nicht immer überstimmend angegeben wird, so war es mir lieb an nenem gut erhaltenem Material der Siboga (s.o.p. 6) und des Fürsten von Monaco die Radienverhältnisse nachzuuntersuchen. Es ergab sich an verschiedenen Species von Pcriphylla eine völlige Uebereinstimmung sowohl untereinander als mit den friheren Angaben: die Rhopalien und je 2 Gonaden liegen interradial entsprechend den Magenflächen und die Tentakel perradial in Radius der Magenkanten und der noch in der Subumbrella deutlichen, stuitzenden Gallertpfeiler. Bei der hier vorliegenden, anders orientierten Form ist eine Wachstumsverschiebung oder eine Zerrung ausgeschlossen, da es sich um $z$ wei, völlig regelmässige Exemplare handelt, die zudem zu den best-conservierten der ganzen Sammlung gehören. Es sind also thatsächlich zwei verschiedene Möglichkeiten der Radienverteilung vorhanden, die sich aber beide von Nausithoc, die 8 Tentakel und 8 Rhopalien besitzt, ableiten lassen; bei Periphylla sind an Stelle der 4 perradialen Rhopalien Tentakel getreten, die in ter radialen Rhopalien geblieben, bei Paraphyllina ist es umgekehrt. Parapliyllina zeigt auch noch in der Structur des Sinneskörpers, bes. durch Vorhandensein eines ectodermalen Pigmentauges mit Linse, sowie in den obenerwähnten Verhältnissen des Schirms, Magens eine grössere Aehnlichkeit mit Nausithoe und Verschiedenheit von Periphylla. Die Aufstellung einer besonderen Familie „Paraphyllinidae”, gleichwertig mit den übrigen von VANHöFFEN unter den Coronaten abgegrenzten Familien (I902, P. 5 I ) clürfte gerechtfertigt sein. Weiter wird man aber in der Absonderung nicht gehen diirfen, trotz der mit den Charybdeiden übereinstimmenden Radienverteilung; dagegen spräche die gemeinsame Ableitung von Nausithoiden, und die typische Coronatenstructur des Schirms. Fur die allgemeinen Beziehungen der Acraspeden untereinander, insbesondere auch für die Frage der Achtoder Vierteiligkeit des Schirms, die nach CLAus für die Beziehungen der Discomedusen zu den Ephyropsiden wichtig ist und die neuerdings auch von KASsiavow erörtert wurden (I9OI, p. 66), erscheint die vorliegende Form von grosser Bedeutung und soll noch an anderer Stelle Besprechung finden. 
1. Paraphyllina intermedia nov. gen. nov. spec. (Taf. II, Fig. Io-i4. Taf. XI, Fig. I06). Stat. 220. Verticalnetz aus 200 M. Ticfe. 2 Exemplare von $8 \mathrm{~mm}$. Schirmhöhe und I5 mm. Durchmesser.

Der Schirm ist flach und gleichmässig gewölbt ohne Scheitelaufsatz; die Kranzfurche ist sehr scharf ausgesprochen und setzt den mittleren, durchaus glatten Teil vom peripheren Pedalien- und Lappenkranz ebenso deutlich ab wie bei Periphylla. Die Pedalien sind von rechteckiger, aber gewölbter Form und durch tiefe Einschnitte getrennt, die nach den Lappen zu auseinanderweichen; die I2 Tentakularpedalien sind gleich breit, die 4 Rhopalarpedalien bedeutend schmäler und zeigen noch einen in den Lappeneinschnitt frei hereinragenden Fortsatz, ehe der eigentliche Sinneskolben beginnt (s. Fig. I I). Die I6 Randlappen sind von spitz-ovaler Form und untereinander fast gleich; die 8 den Rhopalien zugekehrten sind nur wenig schmäler und länger wie die $S$ übrigen. Eine mediale Lappenspange, in der Verlängerung der trennenden Pedalfurche liegend, ist in jedem Lappen, jedoch lange nicht bis zum Ende reichend, sichtbar. Am umgebogenen Schirmrand erscheint daher jeder Lappen durch eine seichte Einkerbung, wie bei den meisten hierher gehörigen Formen, zweigeteilt.

Der Centralmagen ist durch die glashelle Gallerte von der Exumbrella her mit den 4 interradialen Filamentreihen und 4 perradialen Luicken durch die rotbraune Farbe des Entoderms deutlich sichtbar (Fig. I I fil.) Das Magenrohr ist im Vergleich zu Periphylla kurz und hängt in die Subumbrellarhöhle nur bis zum Beginn der Lappenzone herab. Es is von ausgesprochen vierkantiger Form; die 4 perradialen Kanten sind durch Gallertspangen verstärkt, die sich als Träger auch noch auf der Subumbrella deutlich fortsetzen (Fig. Io per). Die rot-braune Färbung ist nur auf die mittlere Partie der interradialen Mundrohrflächen beschränkt; die Seitenteile sind zusammen mit dem Kantenpfeiler rein weiss, ebenso die perradialen Mundecken, sodass das Rohr als ganzes braun und weiss längsgestreift erscheint (Fig. IO u. I I). Das periphere Entoderm ist gänzlich farblos: nur durch die Conservierung treten die Teile der durchsichtigen Gallerte etwas dunkler hervor, aber ohne eigenes Pigment.

Das Canalsystem selbst stimmt durchaus mit dem von den Ephyropsiden im Allgemeinen und von Nausithoe im besonderen beschriebenen Verhältnissen uiberein. Vier interradiale, den Ansatzstellen der Filamente entsprechende Verwachsungen sind deutlich zu erkennen, ebenso I6 Magen-Taschen. In die Lappen treten an jeder Seite getrennte Canäle ein, die sich distal vereinigen.

Die Gonaden zeigen eine Mittelform zwischen denen von Periphylla und Nausithoe resp. Palephyra. Jedes der 8 Säckchen ist am proximalen Ende etwas umgekrümmt und gewulstet; es bleibt also nicht bei der einfachen Ei- oder Schildform von Nausithoe (s. u.) aber es ist auch kein lang ausgezogenes Doppelhufeisen zu erkennen, wie bei Pcriplyylla, sondern eine etwa die Nitte haltende Bohnenform. Je zwei solcher Gonadenbohnen liegen mit den flach-convexen Bogen einander genähert im Interradius; hier ist die Subumbrella tiefer centralwärts, unter die Magenwand zu verfolgen, so dass die Andeutung einer 'Trichterhöhle resp. Subgenitalhöhle entsteht (Fig. Io int.). Die Subumbrella und ihre Muskulatur zeigen ebenfalls ein an Nausithoe, wie Pcriplÿlla anzuschliessendes Verhalten. Ein ziemlich starker Ringmuskel liegt im äusseren 
Bezirk; nach innen sind, besonders zwischen je zwei Gonaden, radiäre Fasern zu erkennen, die nach aussen auseinander liegen; ferner auswärts vom Ringmuskel in jedem Tentakelradius starke Radiärzüge, die nach der Tentakelbasis convergieren und sich auf der Tentakelunterseite ansetzen. Der Ringmuskel erscheint entsprechend den Rhopalien und Tentakeln (nicht den Lappen) in I6 radiäre Felder geteilt. Diese Teilung, sowie das wulstige Hervortreten in die Subumbrella, wird aber nicht durch die Muskulatur hervorgebracht, sondern durch den über derselben befindlichen starken Belag von Nessel- und interstitiellen Zellen. Die Muskelfasern ziehen auch über die Grenzen der Felder hinweg (Fig. Io u. I 2); der Nesselbelag hört aber mit scharfer Grenzlinie auf, so dass 16 gesonderte "Subumbrellarplatten" entstehen. Auch innerhalb jeder Platte zeigen sich einzelne radiärverlaufende hellere Unterbrechungslinien. Distal hat in den 12 Tentakelradien jede Platte noch eine Fortsetzung, entsprechend der erwähnten, zur 'Tentakelbasis laufenden Muskulatur; in den 4 Rhopalarradien, wo sich keine distalen radiären Muskelzüge finden, fehlt auch diese Fortsetzung des Belags (s. Fig. Io).

Die Tentakel sind von typischem Bau. Sie besitzen eine besondere, in der Schirmgallerte aufsteigende Wurzel; an ihrer Basis sind sie etwas aufgetrieben; hier zeigt das Entoderm einen Hohlraum, im Tentakel selbst ordnen sich dessen Zellen zu einer soliden Axe. Der Querschnitt eines Tentakels ist nicht drehrund, sondern ziemlich in axial-abaxialer Richtung zusammengedrückt. Die Länge übertrifft den Schirmradius.

Die Rhopalien sind von denen der typischen Tiefseeformen verschieden, insofern als sie ein sehr grosses, ventral gelegenes Auge mit ectodermalem Pigment und differenzierter Linse zeigen, das sogar noch besser entwickelt ist, als bei Nausithoë und ähnlichen Oberfächenformen. Sonst ist der Kolben, wie das Aufsichtsbild zeigt (Fig. 13) in der gedrungenen Form und der Zusammensetzung dem von Nausithoc sehr ähnlich. Der Otolithensack ist klein (ot) im Verhältniss zur Deckschuppe (sq), die ihn kappenartig umhüllt, der ventrale Bulbus (bu) dagegen sehr gross; er zeigt zwei symmetrische Hälften, die nur wenig nach oben übergreifen, nach unten (innen) aber eine tiefe Bucht lassen, in der ventral nach vorn sich das grosse Auge absetzt. Schon am Oberfächenbild sieht man, dass sich das Ectoderm becherartig in die Tiefe einsenkt, das Pigment (pi) eine halbkugelige Anordnung einnimmt, während eine stark lichtbrechende Linsenmasse (li) den Hohlraum des Bechers ausfüllt und auch nach aussen vorgewölbt erscheint (Fig. I 3 ). Auch das Verhältnis der Schichten, Ectoderm, Entoderm und Gallertlamelle in den iibrigen Teilen kann schon am Durchsichtsbild des gefärbten mikroskopischen Präparats ersehen worden (Fig. I4). Das Epithel auf der Dorsalseite des Otolithensacks (ect!), das in das flache Epithel der Deckschuppe umbiegt, ebenso ein hohes Epithel auf der Ventralseite, auch vor und hinter der Augendifferenzierung, treten wie letztere selbst deutlich hervor. Ob der Endteil des Otolithensacks, der die Concremente trägt, wirklich vom hohlen Teil abgeschnürt ist, scheint nach dem Aufsichtsbild fraglich. Die Abschnürung, die an Radialschnitten erscheint (und auch von Hertwig und Claus bei Nausithoc abgebildet wird), kommt auch hier zum Ausdruck (Taf. XI, Fig. I06), wird aber vielleicht nur durch die Schnittrichtung und die Enge des Verbindungsstückes vorgetäuscht. Eine vollständige Trennung der entodermalen Zellen durch Gallerte kommt jedenfalls nicht zu stande; denn die grosskernigen Zelleì, die die Concremente ausscheiden, stehen durch andere Zellen in Zusammenhang mit den Entodermzellen des proximalen 
Sïckchenhohlraums (Fig. I06). Am wirklichen Schnitt treten die verschiedenen Nervenepithelien (ect!) mit dem basalen Fasergewirr ( $\mathrm{nz}$ ) deutlich hervor, (die dorsalen Fasern des Otolithensacks finden ihre Fortsetzung durch die Gallerte hindurch in einem mächtig entwickelten Aussenepithel (ect!!), das der iusseren Sinnesgrube von Aurclia, Rhisostoma etc. zu vergleichen ist. Die ventralen Fasernetze am Auge und Bulbus mit zahlreichen Kernen hängen ebenfalls zusammen und setzen sich weit proximal fort. Die Zellen, die den Augenbecher auskleiden sind grösser und darum an Zahl viel spärlicher und regelmässiger, als die übrigen Zellen des Sinnesepithels. Die Linse zeigt eine polygonale Structur, die aber wahrscheinlich durch Conservierung hervorgebracht ist. Im Cranzen ist also der Sinneskolben dem von Nausithoe sehr ähnlich, nur noch etwas höher ausgebildet.

Atorellidae Vanh. ig02.

\section{Atorella Vanhöffen Igo2.}

Coronate Meduse mit 6 Rhopalien und 6 Tentakeln, alternierend zwischen je 2 Lappen des Schirmkranzes, mit schwachem Ringmuskel und undeutlichen Tentakeltaschen.

Wegen der cigentümlichen Gliederung des Schirmkranzes ist diese neue Gattung von VAxiöfFEx für ,eine von allen bekannten Arten weit abweichende, höchst interessante Meduse" (1902, 1). 33) gegrindet worden. Der Art, die leider nur in einem „mässig erhaltenen" Exemplar vorhanden war, wurde der Name subglobosa gegeben, wegen der kugelig verdickten, den Schirmkranz allseitig überragenden Centralscheibe. Auch unter dem Sibogamaterial findet sich eine solche eigentiumliche Form nit 6 zähligem Schirmrand wieder. Dieselbe erlaubt, die VANiöfFev'sche Darstellung noch in einigen Punkten zu ergänzen, ist aber, aus grösserer Tiefe kommend, ebenfalls nur mässig erhalten. Es ist daher auch nicht völlig sicher zu entscheiclen, ob es sich um eine neue Species dieser aberranten Gattung handelt. Gerade die Gestalt der Centralscheibe ist hier etwas verschieden, ebenso die Tentakel und einige andere Einzelheiten, die aus der folgenden Beschreibung zu erkennen sind. In diesem Fall witrde ich den SpeciesNamen depressa vorschlagen.

I. Atorella subglobosa Vanh. (Taf. III, Fig. I6, I7, 18).

Stat. I48. I855 1I. Tiefe. Verticalnetz aus I000 MI. I Exemplar von etwa 1,5 cm. Durchmesser. 8 mm. Schirmhölc.

Die Centralscheibe ist hier nur cin halbkugeliger kleiner Aufsatz, der den Schirmkranz nicht überragt; letzterer springt vielmehr noch um so viel Radius vor, als der ganze Durchmesser der Centralscheibe beträgt (Fig. 16). Ringfurche und Pedalfurchen sind nicht sehr tief; letztere setzen sich distal fort und crscheinen am deutlichsten an dem etwas umgebogenen Schirmrand als Kerben in jedem Randlappen. Die Lappen selbst sind oval abgerundet. 
Das Magenrohr ist leider zum grössten Teil abgerissen; doch ist seine Ansatzstelle und die periphere Grenze des Centralmagens durch die Gallerte der Exumbrella hindurch wegen der braunen Färbung deutlich sichtbar (Fig. I6); ebenso deutlich erscheinen die 4 interradialen Filamentbündel, die den dreieckigen Verwachsungsstellen ansitzen (Fig. I 6 fil). Entsprechend den 6 Rhopalien und 6 Tentakeln gehen 12 Taschen vom Centralmagen aus. Was sonst noch vom peripheren Canalsystem trotz des Erhaltungszustandes sichtbar ist, entspricht den Verhältnissen von V'ausithoe etc; die peripheren Enden der benachbarten Taschen vereinigen sich am Lappenrand.

Die Gonaden sind hier, der centralen Einteilung, nicht der des Schirmrandes entsprechend, in der Vierzahl vorhanden und liegen als grosse, bräunliche, gewundene Säcke ganz genau in den Interradien, distal von den Filamentbüscheln, und zwar einzeln in der Subumbrella. VANiöffex hat an seinem Exemplar von $A$. subglobosa ebenfalls nur 4 Gonaden (allerdings Gonadenpaare) gesehen. Er schliesst aber aus deren unregelmässiger Stellung, dass entweder das Exemplar nicht regelmässig entwickelt war, oder 2 Gonadenpaare schon vor der Conservierung zerstört gewesen seien, ü̊d ergänzt deshalb diese zwei in seiner Figur. Die erstere Möglichheit, die der unregelmässigen Entwicklung, führt weiter; denn dann körnte man ja ebenso die Sechszähligkeit des Schirmrands als unregelmässige Ueberentwicklung auffassen (s. u. p. I4). Hier liegen die Gonaden so genau interradial, dass ich deren Vierzahl für das Normale halte. Auch bei Atolla entspricht ja die Einteilung des Schirmrands, der vielzählig ist und schon auf sehr jungen Stadien zahlreiche Antimeren zeigt, wie VANHörfen nachgewiesen hat (1902, p. I6), nicht der centralen Radienverteilung. Ich halte daher seine Ergänzung der Gonadenzahl bei Atorella nicht für nötig. Hier sind dieselben auch insofern auffällig, als es grosse Einzelgonaden, keine Paare sind, die in den Interradien liegen. Abgesehen von der unregelmässig gewulsteten Form des Ganzen lässt sich in jedem einzelnen Wulst eine hellere Mittelzone erkennen, die der Windung entsprechend verläuft. Ob diese der Trennungslinie zweier Gonaden eines Paares oder der von Geschlechtsproducten freien Stelle der Entodermfalte entspricht, vermag ich nicht zu entscheiden, da auch ich dieses einzige Exemplar nicht noch weiter durch Schneiden schädigen wollte.

Die Subumbrella zeigt, soweit ihre Auskleidung erhalten ist, ähnliche Verhältnisse wie Nausithoe. Der Ringmuskel ist, wo vorhanden, sehr schwach entwickelt, was gegenuiber Atolla sehr auffällt; ein besonderer Belag von Subumbrellarplatten konnte nicht erkannt worden.

Die Tentakel sind kürzer als der Schirmradius; an der Basis kaum verdickt, an der Spitze plötzlich abgerundet. Wie VAshörfen angiebt, (1902, p. 33): „tritt die Entodermasse mit kurzem Zapfen in die Schirmgallerte ein". Dieser blind endende Zapfen umgreift noch die Tentakelbasis selbst (Fig. I 7). Der entodermale Hohlraum setzt sich auch noch in den Anfangsteil des Tentakels fort; die Kerne zeigen hier eine regelmässige Reihenordnung; weiter peripher aber bilden die Zellen eine solide Axe, die im Querschnitt aus mehreren Zellen mit unregelmässig liegenden Kernen besteht.

Die Rhopalien (Fig. I8) zeigen ähnliche Form wie bei Atolla und Vausithoc. Die Schuppe ist nicht, wie bei Periphylla, schmal und der ganze Kolben gestreckt, sondern letzterer gedrungen, und die Schuppe umgreift fast den ganzen Kolben, auch ventral. Ein ventraler 
Bulbus (bu) mit differenziertem Ectoderm und dunklerem Entoderm ist deutlich wahrnehmbar: ehenso innerhalb der Schuppenwölbung der kleine Endsack (ot) mit den Otolithen.

\section{PEricolpidae Haeckel is79.}

Pericolpa Haeckel is 79.

Sens. em. Vanhöffen 1902.

Coronate Meduse mit 4 Rhopalien und 4 Tentakeln, alternierend $z$ wischen 8 Randlappen.

In Bezug auf Antimerenzahl und Einteilung des Schirmrands ist das Genus Pcricolpa die einfachste Coronatenform. Nausithoc, Palephyra und Zoncphyra zählen i6 Randlappen und 16 Randanhänge; ebenso auch Periphylla und Paraphyllina; nur sind bei den ersten 3 Genera von den Randanhängen 8 Tentakel, \& Sinneskolben, bei den letzteren I 2 'Tentakel und 4 Sinneskolben. Bei Atolla wird diese Gesammtzahl noch wesentlich ïberschritten, und nur bei Atorclla bleibt sie mit 6 Tentakeln und 6 Sinneskolben zwischen I 2 Randlappen dahinter zuriick. Das Zahlenverhältnis: 4 Rhopalien und 4 Tentakel zwischen 8 Randlappen kommt sonst in der ganzen Gruppe nicht vor, und selbst die Ephyralarve, die einfachste Form, in der alle Discomedusen zuerst erscheinen, hat bereits I6 Randlappen wie Nausithoc. Es ist daher die Gattung Pericolpa für die verwandtschaftlichen Beziehungen der Scyphomedusen iiberhaupt von grosser Wichtigkeit, und sie wird von HaEckel, Claus, Kassianow verwertet zur Anknüpfung an die primitiveren und teilweise festsitzenden Formen. In wirklichen Exemplaren ist sie aber ausser von HAECKEL bisher nicht bekannt geworden. Es konnte daher der Verdacht ausgeprochen werden, dass es sich vielleicht nur um ein Jugendstadium handle, doch bemerkt VANHöFFEN hierbei mit Recht (1902, p. 50), „dass eine Vermehrung der Metameren des Schirmkranzes bei Coronaten nicht vorkommt"; die von der ValdiviaExpedition gefundenen teilweise recht jungen Exemplare von Atolla und Periphylla wiesen bereits die volle Antimerenzahl auf. Dasselbe kann ich von kleinen Atollen der Siboga- und anderer Sammlungen bestätigen; ausserdem besitzt die hier vorliegende ansehnliche $P$ cricolpa reife pralle Gonaden. In obigem Sinn ist die Gattung Pcricolpa gleichbedeutend mit der Hatckel'schen Familie der Pericolpiden, die ausser Pcricolpa noch Pcricrypta einbegreift. V.NHöfres hat mit Recht beide Genera zusammengezogen, da sich die Merkmale ungezwungen als Altersunterschiede deuten lassen (I902, p. 50). Bei der kleineren Form, die auch weniger Gastralfilamente hat, reichen die interradialen Trichterhöhlen nicht so hoch hinauf und die Taeniolen sind infolge dessen solide, nicht hohl. Die 3 Arten scheinen mir auf 2 zuriickfiihrbar, indem galea mit quadrigata zusammenfält. Die vorliegende Form rechne ich der Schirmform wegen einstweilen zu campana; in den Breiteverhältnissen von Tentakelpedalien und Rhopalarpedalien, sowie der Tentakellïnge stimmt sie allerdings mehr mit galca ïberein. Die Speciestrennung ist nur eine vorläufige. Das vorhandene einzige Exemplar, das sehr stark contrahiert und etwas unregelmässig entwickelt ist (s. u. p. I4), erlaubt keine Entscheidung. erfordert aber als einziges seit den HaEcken'schen besondere Beschreibung. 
I. Pericolpa campana mihi. (Taf. III, Fig. I9, 20, 21, 22:

I'ericrypta campana Haeckel i 879 .

Stat. I48. I $8_{55}$ M. Tiefe. Verticalnetz aus I000 M. I Exemplar von I,5 cm. Höhc, davon etwa $\$ \mathrm{~mm}$. Lappenkranz, $57 \mathrm{~mm}$. Centralscheibenradius und $1,5 \mathrm{~cm}$. Schirmdurchmesscr.

Der Schirm ist ungefähr ebenso hoch als breit und die ganze Meduse erscheint durch die dicke gewölbte Gallerte der Centralkuppel einerseits, sowie durch die eingezogenen Randlappen andrerseits beinahe kugelig (Fig. I9 und 20). Die Ringfurche (fos) ist tief eingeschnitten; ebenso die davon radiär ausgehenden Furchen zwischen den Pedalien. Zwischen den 4 Rhopalarund den Tentacularpedalien ist kein wesentlicher Breitenunterschied; was mit galea, nicht aber mit campana übereinstimmen würde. Doch sind auch die für letztere und für die Pericolpiden iiberhaupt von HAECKEL angegebenen Stellungsverhältnisse der Lappen hier nicht zu erkennen. Die Pedalien sind nicht gegeneinander verschoben, wodurch ein Alternieren wie bei Atolla vorbereitet würde, sondern stehen gleichmässig und auf gleicher Höhe neben einander am Schirmrand. Auch zeigt sich kein besonderer Fortsatz für das Rhopalium, sodass hier wenigstens die von Kassianow (1901) an die Gruppe angeknuppten Homologien nicht zutreffen. Auch die Lappen erscheinen untereinander durchaus gleichwertig und gleich in Gestalt und Grösse. In jedem Pedalium ist eine mittlere, nicht bis zur Ringfurche reichende Trennungslinie zu erkennen, so dass es, wie auch auf der HAECKEL schen Figur (1879, Taf. XXIII) ersichtlich, in 2 Hälften zerfällt. In der Verlängerung der eigentlichen Pedalfurchen sind auf den Lappen die Lappenspangen zu erkennen.

Der Magen sitzt mit breiter Basis der Subumbrella an, ohne einen. Stiel-Fortsatz oder auch nur helmförmigen Aufsatz in die Schirmkuppel zu entsenden. Vier starke Filamentbiischel, jedes mit mindestens 30 Filamenten, sind durch die Gallerte hindurch durch die dunkelbraune Farbe sichtbar, ebenso wie der Centralmagen. Das Nundrohr war am vorliegenden Exemplar nicht erhalten, dagegen die 4 perradialen Ostien zwischen den Filamenten deutlich sichtbar. Vom peripheren Canalsystem ist, soweit es erhalten ist, noch nachzuweisen, dass den 8 Hauptradien entsprechend 8 grosse Taschen vorhanden sind, die sich am Lappeneinschnitt gabeln. Die Gabelenden zweier benachbarten Taschen bilden dann, nur durch eine kleine Venwachsungsstelle getrennt, distal vereinigt den entodermalen Hohlraum des Lappens.

Die Gonaden (gon) liegen als dunkle, unregelmässig gewulstete Schilder im mittleren Teil der Subumbrella. Am vorliegenden Exemplar sind 7 Schilder vorhanden, ein achtes ist nachweislich abgerissen. Ihre Verteilung ist ungleichmässig, schon durch die Unregelmässigkeit am Schirmrand; ob sie zu je zweien genähert sind, und in welchen Radien konnte deshalb und wegen der starken Contraction nicht entschieden werden. Aus gleichen Grinden kann auch nicht mit Sicherheit gesagt wurden, ob wirklich, wie in der Zeichnung angegeben, die Tentakel und nicht die Rhopalien in den Radien der Filamentbischel liegen.

Der Subumbrellarmuskel (m cir) ist in seinem ringformigen Teil gut erhalten und zeigt eine starke Ausbildung. An der Tentakelbasis sind Radiärzuige nachweisbar.

Die Tentakel selbst sind ziemlich kurz, kiirzer als bei allen von HAECkEL angefiihten Arten; zeigen eine deutliche Wurzel in der Schirm-Gallerte und eine solide Axe.

Die Rhopalien haben den typischen Bau. Im Aufsichtsbild resp. optischen Schnitt 
(Fig. 21) zeigt sich ein entodermaler Axencanal mit einer ventralen Aussackung, dem Bulbus, der auch noch rechts und links auf die Dorsalseite heribergreift und durch dunkleres Entoderm, sowic höheres Ectoderm ausgezeichnet ist; ferner der kolbige Endteil, dorsal von der Deckschuppe (sq) bedeckt und nur durchschimmernd (Fig. 2I), ventralwärts mit den Otolithen deutlich erkennbar (Fig. 22 ot). Ein ventraler Ocellus wie bei Nausithoc, Paraphyllina fehlt.

Das vorliegende Exemplar zeigt insofern eine Unregelmässigkeit, als am Schirmrand in einem Quadranten zwei Tentakel und zwei Lappen zwischen den Rhopalien stehen (Fig. 20 t!). Dass es sich hierbei um keine Wachstumsvermehrung der Lappenzahl handelt, sondern um eine Anomalie, geht ausser den für junge Periphyllen und Atollen erörterten Thatsachen (s. u.), auch hier aus der Unregelmässigkeit in Grösse und Form, sowie daraus hervor, dass es nur in einem Quadranten der Fall ist. Auch an eine Annäherung an die 6-zählige Atorella, die von gleicher Fundstelle ist, und sonst im Bau vielfache Aehnlichkeit aufweist, kann wohl kaum gedacht werden, schon der Zahl und Lage der Gonaden wegen nicht, von andern Unterschieden ganz abgesehen. Und die letztere als iberentwickelte Pericolpa aufufassen, ist ebensowenig möglich, schon im Hinblick auf den doppelten Fiund von der Valdivia und der Siboga. Andere vier-zählige Formen wirden jetzt, nachdem die Organisation der 8-zähligen wie Pcriphylla, Nansithoc, Paraphyllina etc. besser bekannt ist, von besonderem Wert sein, auch für weiter ausgreifende Vergleiche.

Collaspidae Haeckel is79.

Atolla Haeckel 1879 .

Sens. ampl. Fewkes I 886 .

Die Gattung Atolla, bisher in ihren Angehörigen eine Seltenheit und nur von den grösseren Expeditionen bekannt, wurde zuerst von HAECKEL nach Challengermaterial aufgestellt (1879) und in ihrer Organisation beschrieben, durch FEwkEs in ihrer Systematik bereichert (I 886), auch unter dem Acalephen-Material der Plankton-Expedition von VAxiöffex (IS92) aufgeführt und sodann am Albatrossmaterial von mir in Bezug auf einzelne Organisationsverhältnisse, bes. Canalsystem und Gonaden studiert (1897); auch unter den Tiefseezügen des Fürsten von Monaco habe ich sie erwähnt (I899). Neuerdings konnte VANröffex an dem schönen Naterial der Valdivia, das 52 Exemplare aus dem Atlantischen, Antarktischen und Indischen Ocean enthält, "die frihheren Beobachtungen ergänzen und die Beziehungen der unterschiedenen Arten zu einander prififen" (1902, p. 5). Auch von der Siboga wurden 9 Atollaexemplare ron 6 Fundstätten aufgezogen, die mir ebenfalls erlauben, auf einige Punkte der Organisation. und Systematik einzugehen.

VANHöFfex hat den morphologisch wichtigen Nachweis erbracht, dass im Lauf der Entwicklung hier keine Vermehrung der Teilstiicke rorkommt, sondern kleine Exemplare bereits die Antimerenzahl von 20 und mehr aufwiesen. Auch am hier vorliegenden Material kann ich das bestitigen; die kleinsten Exemplare von $12 \mathrm{~mm}$. zeigten dieselbe Antimerenzahl wie die von 
ebensoviel cm. (s. Tabelle). Die Zahl selbst ist laut den Vanuöffex'schen instructiven Tabellen ziemlich schwankend, auch bei den Individuen derselben Art: er und Fewres haben bis zu 29 Antimeren, HaEckel und ich 32 und mehr gefunden. Hier ist die Maximalzahl ebenfalls 29, zufällig gerade am kleinsten Exemplar; die Ninimalzahl 20.

Der M a gen ragt an meinen grössten Exemplaren noch kuppelartig in die Centralscheibe hinein, an den andern liegt er der Subumbrella flach an und die Centralscheibe selbst ist niedrig. Die Wölbung der Exumbrella ist laut VANHöFfEx das normale Verhalten, das Gegrenteil durch Conservirung bedingt.

Weiter ist es VANIÖFFEN möglich gewesen, das wegen der schlechten Erhaltung bisher strittige periphere Canalsystem nachzuweisen. Ich habe die radiären breiten Tentacular- und schmalen Rhopalarcanäle noch mit ihrer Gabelung nur bis in die Lappen verfolgen können und hervorgehoben (1897, p. 72), "dass es von Interesse wäre zu entscheiden, ob die Gabelenden blind aufhören, wie es HAECKEL abbildet.... oder ob sie zu einem Festoncanal vereinigt sind". Eine solche Verbindung hat VANHöFFEN durch Einblasen von Luft nachweisen können (1902, p. 17). Ebenso habe ich diesen die Gabelenden der Rhopalar- und Tentacularcanäle verbindenden Festoncanal durch das wohlerhaltene braune Pigment an einem besonders schönen Exemplar aus den Sammlungen den Fürsten von MIonaco zeichnen können (bisher noch nicht veröffentlicht), und finde auch hier die gleichen Verhältnisse (Fig. 23). Man sieht, wie auch VANHÖFFEN angiebt, daraus ferner, dass sich der schmale Rhopalarcanal noch in das Sinnesorgan selbst fortsetzt, also dreigabelt, während an der Tentakelivurzel kein solcher medialer Canal, sondern nur ein entodermaler, in die Axe verlaufende Zellstrang zu erkennen ist. Weiterhin ist, was VAxHöFFEx nicht erwähnt, zu bemerken, dass die Tentakulartasche nicht in ihrer ganzen Breite wegsam bleibt, sondern an ihrem Abgang vom Ringsinus ein mediales, am Abgang der Seitencanäle zwei seitliche Verlötungsfelder zeigt; dadurch wird im Aufsichtsbild der Modus der Verzweigung sehr beeinflusst (Fig. 23), so dass man fast 3 parallele schmale zum Doppel-Lappen laufende Radialtaschen und eine breite nach dem Tentakel sich verlierende Schleife unterscheiden kann. Noch mehr tritt das an alten Exemplaren hervor.

An den Rhopalien, die noch von HAEciel als rückgebildet bezeichnet wurden, habe ich s. Zt. bereits Otolithensack, Deckschuppe, Sinnespolster und Bulbus nachweisen (I 897, p. 75) aber den feineren Bau der Erhaltung wegen nicht studieren können. Nunmehr wird durch V.1xHöfFex die erwünschte mikroskopische Darstellung gegeben (I902, Taf. VII, Fig. 54-68). „Ein ectodermaler Ocellus, wie er Vausithoe etc. zukommt, fehlt hier ebenso wie bei Pcriphyllar; zum Unterschied von letzterer ist der Otolithensack kleiner im Verhältnis zur Deckschuppe.... und das Sinnespolster der Unterseite greift nicht so weit herauf" (1900, p. 279). Ferner wurden durch VAxHöfFEx grosse "Hauptganglien” rechts und links an jedem Rhopalium gefunden und als Centralorgane des Nervensystems gedeutet. Mein eigenes Material erlaubt mir leider nicht das Studium an Schnittserien.

Meine Darstellung des Gonadenbaus hat VANrökFex durch Beschreibung an jungen Exemplaren ergänzt; an solchen findet er, wie ich es bereits fir P'eriphylla nachgewiesen, dass die Geschlechtsproducte noch in der Gallertschicht liegen. Ich habe daraus s. Zt. den Schluss gezogen, dass die Genitalproducte auch hier, wie im Tierreich mehrach nachgewiesen, keinem 
bestimmten Keimblatt angehören, sondern nur in den Verband des Entoderms rücken, so wie bei Craspedoten ins Ectoderm, bei Spongien ins Füllgewebe. VAxhöfrex hält die Annahme eines solchen "besonderen indifferenten Gewebes..... nicht für nötig" (p. 20), sondern glaubt, dass bei allen Coelenteraten die Keimzellen ectodermalen Ursprungs sind „und nur behufs besserer Enährung ins Entoderm einwandern". Ich halte gerade die VANıÖFfEv'sche Annahme für weiter hergeholt und muss an meiner früheren Ansicht festhalten, die auch der mittlerweile allgemeiner gewordenen Anschaung von der Stellung der Geschlechtsorgane zu den Keimblättern entspricht (s. Kórschelt und Heider $2^{\text {te }}$ Aufl. igo2, Délage und Hérouard, Coelenterés).

Ein bisher vollständig unbeachtetes "eigentünliches Organ" hat. VANHöfFEs in der Subumbrella gefunden, nämlich an jeder der 4 perradialen Magenecken zwei stark vortretende Farbflecke, deren Pigmentierung sowohl dem hohen Ectoderm wie dem Entoderm zukommt. Er vermutet darin eine Art Excretionsorgan. Ich habe diese Flecke ebenfalls, zwar nicht bei allen, aber gerade bei gut erhaltenen jungen Exemplaren gefunden (Fig. 4 und Io8). In Bezug auf ihre Deutung vermag ich nichts Sicheres zu sagen; vielleicht sind sie ähnliche Gebilde wie die Subumbrellarbläschen der Linergiden. Hoden, was HAECKEL bei letzteren vermutet, sind es hier keinesfalls.

W'as die Systematik innerhalb der Gattung Atolla betrifft, so hatte ich s. Zt. nur nach der Litteratur ohne eigenes Material die Merkmale der beschriebenen Arten zusammenstellen müssen und zwei neue Spezies hinzugefügt. VANHÖFFEN hat nunmehr diese Merkmale, nicht aber die Arten, verworfen und neue Unterscheidungen angegeben. Da dieselben aus dem grossen Material von 52 selbst conservierten Exemplaren inductiv gewonnen sind, zu 5 der bekannten Species führen und zudem leicht erkennbares verwerten, so wird man seiner Einteilung gerne folgen. Eine Zwischenzone zwischen Centralscheibe und Pedalienansatz fehlt laut VANHöFfex bei keiner Atolla-Species; er teilt daher die radiären Kerben bei meiner A. gigantca dieser Zwischenzone und nicht der Centralscheibe, wie bei $A$. Vorrilli zu; dagegen ist ihm meine A. Alcxandri als Art zweifelhaft. Ich kann VAxhöffex zugeben, dass die Grösse der Centralscheibe i. V. zum Schirmkranz je nach der Erhaltung wechseln kann; aber ein so extremes Verhältnis, wie bei A. Alexandri, wo die Pedalien und Lappen nur verschwindende Anhänge i. $V^{\top}$. zur breiten flachen Scheibe darstellen, wird durch keine Schrumpfung oder Conservierung cintreten können. Von A. Agassiz und MAYER ist diese Form - allerdings ohne Abbildung auch seither wieder erwähnt worden (1902).

Die hier vorliegenden frormen gehören zu keiner von beiden mir bisher in natura bekannten Arten, sondern sämmtlich zu der ron der deutschen Expedition im Indischen Ocean neu gefundenen A. Valdiria. Sie besitzen eine glatte nicht gekerbte Centralscheibe, deren Durchmesser zum gesammten Schirmclurchmesser sich etwa wie i : a verhält. Diese Merkmale sind ihnen noch mit $A$. Bairdi gemeinsam; doch unterscheiden sie sich von letzterer, die ich an anderem Material vergleichen konnte, sowie ron allen Atollen, durch das von VANHöffex angegebene Merkmal der "grossen, den ganzen Raum zwischen den Ausschnitten des Magenkreuzes erfillenden Septalknoten". Eine instructive Ansicht dieser grossen, bis zum Centrum vorspringenden Septalknoten giebt ein Exemplar, bei dem das Magenrohr selbst abgerissen und (ler Boden darum um so besser zu sehen ist (Taf. I, Fig. 3). 
I. Atolla Valdiviae Vanhöffen igoz.

(Taf. I, Fig. 3, 4. Taf. III, Fig. 23. Taf. XII, Fig. IOS).

Folgende Tabelle zeigt wie bei VANHöfFEx, die erbeuteten Exemplare nach der Grösse geordnet, nebst Antimerenzahl und Angaben über Fundort und Tiefe.

\begin{tabular}{|c|c|c|c|c|c|c|c|c|c|}
\hline$x^{0}$ & $\underset{m}{S}$ schir & $\begin{array}{l}\text { mendurcli- } \\
\text { esser. }\end{array}$ & Centrai & ischeibe. & $\begin{array}{l}\text { Antimeren- } \\
\text { zahl }\end{array}$ & Station. & Geogr. Preite und I.ảngec. & Gelocte 'liefe. & Tiefe des Fanges. \\
\hline I. & I 2 & $\mathrm{~mm}$. & & $\mathrm{mm}$. & 29 & 230 & $3^{\circ} 5 S^{\prime} \quad$ S., $128^{\circ} 20^{\prime} \quad 0$. & - & aus $2000 \mathrm{M}$. \\
\hline 2. & & $\pi$ & 7 & , & 20 & 203 & $3^{\circ} 32^{\prime} .5$ S., $124^{\circ}$ I $5^{\prime} .5 \mathrm{O}$. & $\overline{4892}$ M. & $\begin{array}{l}\text { aus } 1500 \mathrm{MI} . \\
\text { Vertikalnetz. }\end{array}$ \\
\hline 3. & I $S$ & " & 8 & $n$ & 20 & 203 & $3^{\circ} 32^{\prime} .5$ S., $124^{\circ} 15^{\prime} .5 \mathrm{O}$. & & \\
\hline 4. & 20 & » & 8 & $\pi$ & 23 & $46^{2}$ & $8^{\circ} \quad 0^{\prime} .5$ S., $118^{\circ} 34^{\prime} .7$ O. & 1600 & $\begin{array}{l}\text { Trawl, gleiche } \\
\text { Tiefe wie Lot. }\end{array}$ \\
\hline 5. & 32 & r & I6 & $n$ & 22 & 122 & $1^{\circ} 58^{\prime} .5$ N., $125^{\circ} 0^{\prime} .5 \mathrm{O}$ & $1264-$ I $165 n$ & $\begin{array}{c}\text { Trawl, gleiche } \\
\text { Tiefe. }\end{array}$ \\
\hline 6. & 35 & . & 20 & " & 24 & 173 & $3^{\circ} 27^{\prime} \quad$ S., I $3 \mathrm{I}^{\circ} \quad \mathrm{O}^{\prime} .5 \mathrm{O}$. & $567 \pi$ & $"$ \\
\hline 7. & 42 & n & 22 & » & 20 & 173 & $3^{\circ} 27^{\prime} \quad$ S., $13 \mathrm{I}^{\circ} \quad 0^{\prime} .5 \mathrm{O}$. & $567 \pi$ & " \\
\hline S. & 65 & $n$ & 35 & , & 24 & 262 & $5^{\circ} 53^{\prime} .8$ S., $132^{\circ} 48^{\prime} .80$. & 560 & , \\
\hline 9. & $\mathrm{~S}_{2}$ & , & 42 & , & 24 & 262 & $5^{\circ} 53^{\prime} .8$ S., $132^{\circ} 4 S^{\prime} .8 \mathrm{O}$ & $560 \%$ & $r$ \\
\hline
\end{tabular}

Alle Atolla-Exemplare stammen also aus grösserer Tiefe. Aehnliches gilt noch von einer weiteren Anzahl Acraspeden aus dem Siboga-Material, die ich hier zusammenstelle.

\begin{tabular}{|c|c|c|c|c|}
\hline & Station. & Geogr. Breite und Lange. & Meerestiefe. & Tiefe des Fanges. \\
\hline Periphylla dodecabostricha & 230 & $3^{\circ} 58^{\prime} \quad$ S., $128^{\circ} 20^{\prime} \quad$ O. & 2000 & aus $2000 \mathrm{M}$. \\
\hline Pericolpa campana & 148 & $0^{\circ} 17^{\prime} .6$ S., $129^{\circ} 14^{\prime} .5 \mathrm{O}$. & 1855 & aus 1000 \\
\hline Atorella subglobosa & 148 & $0^{\circ} 17^{\prime} .6$ S., $129^{\circ} 14^{\prime} .50$. & 1855 & aus 1000 \\
\hline Paraphyllina intermedia & 220 & $6^{\circ} 2^{\prime} \quad$ S., $123^{\circ} 57^{\prime} .7 \mathrm{O}$ & 278 & aus 200 \\
\hline
\end{tabular}

Die Frage, ob es Tiefseemedusen überhaupt giebt, war längere Zeit strittig und wurde gegen HaEckel von Fewres (1888) direkt verneint. Noch bei den Medusen des Albatross habe ich (1897, p. 65) für Periphyllen bemerkt, dass, sie in sämtlichen I 3 Fällen a u s ${ }^{1}$ ) grösseren Tiefen $(700-1670)$ kamen, und dass im Gegensatz dazu an flachen Stellen keine Periphylliden gefischt wurden. Aber in sehr vielen andren Zügen aus grossen Tiefen kamen keine vor und ebensowenig im geschlossenen Tiefennetz. Wir haben also einstweile ${ }^{1}{ }^{1}$ ) kein Recht, diese Tiere als Tiefseemedusen anzusprechen". So wie VAxiöfFen diesen meinen Einwand anfuhrt (r 902, p. 22) und dabei vor dem Schlusssatz meine Constatirung einschiebt, dass auch Atolla nur in Zügen aus mindestens 555 Faden vorkam, erscheint meine negative resp, abwartende Folgerung in der That „überraschend". Mir schien es nur damals, wie das Wort „einstweilen” zeigt, bei dem Fehlen der Medusen in den Schliessnetzen und bei der Unsicherheit der Vergleichsmethode der offenen Verticalnetzfänge, geraten, eine grössere Menge von Befunden noch abzuwarten, ohne

I) Der gesperte Druck ist erst diesmal angewandt.

SINOGA-EXIEDITIE XI. 
dass ich ein Gegner der Ansicht von wirklichen Tiefseemedusen gewesen wäre. Ich selbst habe, sobald mir eine Reihe weiterer solcher Fänge aus dem Material des Fürsten von Monaco vorlag, in einer Mitteilung (I899), die VANHöFFEN wegen der etwas versteckten Publication wohl entgangen ist, mich ausdrücklich zu dieser Ansicht bekannt. Ich habe daselbst hervorgehoben, dass in den Oberflächenzügen nur gewöhnliche Formen vorkommen, dass aber Pcriphylla, Atolla nur in den Fängen aus grösserer Tiefe enthalten sind. Ferner erwähnte ich das Vorkommen einer Cunina aus $78 \mathrm{r}$ M.; die sich vor ihren transparenten Verwandten der Oberfläche durch die purpurviolette Färbung auszeichnet, so dass auch bei den Medusen der verschiedensten Gruppen dies als Tiefseefärbung angesehen werden müsse. Diese Befunde wurden auch vom Fuirsten von Monaco selbst in einer Mitteilung vor dem VII. internat. Geographencongress (I 900) hervorgehoben. Dadurch, sowie durch die noch viel grössere Zahl der Valdiviafänge (IS99) ist die Existenz von wirklich in der Tiefe lebenden Medusen sichergestellt, und auch die hier vorliegenden Fänge, denen sich noch bei den Craspedoten weiteres Material zugesellen wird, liefern eine schöne Ergänzung dazu. Man kann ebenfalls sagen: „Toutes les formes non banales viennent d'une profondeur assez grande". (Pr. de Monaco I goo).

Eine weitere Frage ist die, ob diese Tiefseemedusen oberhalb des Grundes oder in der intermediären Wasserschicht, weit vom Grund und der Oberfläche entfernt, leben. Für Atolla ist nach den Tabellen der Valdivia (1902, p.6) wohl letzteres anzunehmen; auch hier zeigt ein Fang (Stat. 203) eine gelotete Tiefe von ïber 4890, und das Netz mit Atolla kam nur aus 2000 M. herauf, also fast 3000 M. vom Grunde entfernt, wie es auch VANHöfFen bemerkt (I902, p. †). Für Pcriphylla, die vielleicht, wie ihr Stielcanal andeutet, ein festsitzendes Scyphostomastadium hat, scheint ein solches intermediäres Vorkommen fraglicher; vielleicht lebt sie direkt oberhalb des tieferen Grundes.

In der Ausprägung der purpurvioletten Färbung bestehen graduelle Verschiedenheiten, die vielleicht mit solchen der Aufenthaltstiefe correspondieren. Atolla soll sogar auf der Exumbrella ein pigmentirtes Epithel besitzen, der Magen ist tief dunkel. Periphylla hyacinthina hat Magen, sowie Canäle braunviolett gefärbt; $P$. dodecabostricha nur den Magen; die Gonaden schimmern durch die glasshelle Gallerte durch. Bei Parapliyllina intermedia, die nur aus 200 M. bei $278 \mathrm{M}$. Grundtiefe aufgezogen wurde, ist nicht einmal der ganze Magen rotviolett gefärbt, sondern nur 4 interradiale Seitenstreifen. Auch besitzt diese Form noch grosse und gut entwickelte Augen mit Linse.

Nausitioidate Haeckel i 879.

Sens. emend. Vanh. Ig0z.

Nausithoe Köllik. 1853 .

VAxHöfFEN, der zuerst die bei HAECKEL so weit aus einanderstehenden „Peromedusen” mit einem Teil der Discomedusen vereinigt hat, hat sich dabei besonders an den Vergleich von Pcriphylla und Nausithoe gehalten; die Mittelstellung, die Nauphanta einnimmt, wurde auch von mir erörtert (IS92, p. S2) und dieselbe „als diejenige Ephyropside bezeichnet, die noch am 
nächsten mit den typischen flacheren Formen, Nausithoidae, verwandt ist, und von diesen zu Atolla hinüberleitet". Die wichtige Umordnung VAnröfrex's im System der Discomedusen, nämlich die Aufstellung der Gruppe der Coronaten, wird wohl allseitig anerkannt, wenn auch - Modificationen im einzelnen vorgenommen werden. Neuerdings hat VANHöFfEx selbst die vermittelnde Gattung Nauphanta direkt mit Nausithoc vereinigt, von der sie bisher durch dic Sculptur der Exumbrella und die Vermehrung der Lappentaschen verschieden galt. Craus hat nämlich schon für Nausithoe nachgewiesen, dass in jeden Randlappen nicht eine, sondern zwei Taschen eintreten; Ringfurche und Pedalien kommen auch bei Nausithoe vor, wie VANHöffen bereits in den Acalephen der Plankton-Expedition betont, und die Gattung Nauphanta muss also aufgegeben werden (VANHöFFEN 1902). Auch bei den hier vorliegenden stattlichen Nausithoc-Exemplaren, die die bisherigen Arten an Grösse beträchtlich übertreffen, kann ich bestätigen, dass die Ringfurche und Pedalien, die bei den kleinen flachen $N$. punctata leicht ubbersehen werden, hier fast so deutlich wie bei Atolla-Exemplaren sind, und ferner, dass sich in jedem Lappen auf Schnittserien eine Cathammenstelle nachweisen lässt, so dass man, wenn man will von 2 Canälen reden kann, die in ihn eintreten. Die grösste Ausdehung des Lappens allerdings, das ganze Distalende des Entodermes ist wieder einheitlich. MAyer (1902, p. I 55) spricht allerdings darum nur von i6 Lappentaschen, zählt also auch am Magen selbst nur Radialtaschen in den Radien der Sinnesorgane, ohne die Tentakeltaschen zu bemerken und hat seine Darstellung offenbar nur nach dem Oberflächenbild an Leben gemacht. So viel ich aber auf Schnitten bemerken kann, stimmt die MAYen'sche Nausithoe, zu der mein Material gehört, hierin völlig mit der mediterranen überein; liefert also keinen Grund für die Sonderstellung von Nausithoc gegen Nauphanta.

Ob das Genus Nauphanta nicht trotzdem auf Grund anderer Unterschiede aufrecht zu erhalten wäre, kann erst bei weiterem Material von bisherigen Nauphanta-Species erörtert werden. Es ist auch für die vorliegende Form ohne Belang, weil sich dieselbe von den Arten, auch nach VANHörFes'scher Zusammenfassung unterscheidet. Es fallen von Nauphanta-Arten ausser $N$. polaris auch VANHöffen's eigene $N$. Vettoris Pisani unter Nausithoe punctata, die ausserordentlich weit verbreitet ist (VANHÖFFEN 1902, p. 29); $N$. challongeri Haeckel und $N$. albatrossi Maas bleiben auch im andern Genus als wohl charakterisirte Species bestehen. Dazu kommt noch eine neue, ebenfalls sehr prägnante Tiefseeform VAniörfex's, $N$. mbra und die gleichzeitig von Agassiz und Mayer beschriebene grosse Oberflächenform $N$. picta (1902). Wie sich dazu die VAnHöfFEx'sche frühere Art $N$. Clausi stellt, von der ihm nur ein Exemplar mit sehr kleinen Gonaden vorlag, ist mir nicht ganz klar.

1. Nausithoe picta A. Ag. und A. G. Mayer I902. Sens, emend. Maas.

(Taf. I, Fig. 5, 6, 7,8 ).

Mit glatter Centralscheibe und breiten, distal zugespitzten Rand. lappen, mit kurzen an der Basis sehr verbreiterten Tentakeln. Mit sehr grossen, schildförmigen, proximal verbreiterten Gonaden. Farbe: Gonaden und Ocelli rotbran bis carminrot, Gastralfilamente bläulich. 
Stat. IO3. 2 Exemplare.

Stat. I06. I

Stat. Iog. 7

Stat. I 44. I

Sitat. 252 . i

Stat. 282.3

Sulu.

Die Identificirung geschicht versuchsweise nach der Abbildung, die 1. c. nach dem Leben gegeben wird; aus der Mayer'schen Beschreibung lässt sie sich kaum folgern; denn wir erfahren da fast nichts, was nicht für jede Nausithoe oder sogar jede Ephyropside gelten wiirde, nämlich dass 8 Tentakel und Sinnesorgane alternirend mit i 6 Lappen vorhanden sind und „dass der Mund im Centrum der Subumbrella gelegen ist"; und was über des Canalsystem bemerkt wird, ist sogar unrichtig.

Der Schirm erscheint ziemlich gewölbt, nicht flach wie MAYEer schreibt; dadurch und durch die scharfe Kranzfurche hebt sich die Schirmkuppel sehr deutlich von der Pedal- und Lappenzone ab. Bei der oralen Ansicht kommt diese Scheidung wegen des Muskelrings und der Subumbrellarplatten, und bei der aboralen wegen der Durchsichtigkeit der Meduse nicht so zum Ausdruck; im Profil aber ist sie sehr deutlich ausgesprochen. Irgend welche Structur oder Punktirung ist auf der Exumbrella nicht wahrzunehmen.

Dic Randlappen zeichnen sich durch ihre breitbasige, etwas ausgeschweifte und distal zugespitzte Form aus. Man sieht auch deutlich, dass sie zu je zweien im Radius eines Rhopaliums zusammen gehören; der Einschnitt für den Tentakel ist viel weiter als der für das Rhopalium. Die Tentakelbasis ist sehr breit und der Tentakel verjüngt sich nur sehr allmählich, so dass er im Ganzen durch Kürze und Dicke gegenüber den Gebilden der $N$. prnatata auffält.

Besondere Beachtung verdienen die „Subumbrellarplatten". So nenne ich die r6 viereckigen Felder, die als dicker ectodermaler Belag von Nessel- und interstitiellen Zellen auf dem Kranzmuskel in der Subumbrella liegen. Die Felderung wird nicht durch die Muskelverteilung hervorgebracht, denn der Kranzmuskel ist continuirlich und zeigt seine circulären Fasern gerade in den Unterbrechungen der Subumbrellarplatten um so deutlicher (s. Fig. 8). Cuaus hat diesen "dichten von Cnidoblasten erfiillten Zellbelag" bei $N$. punctata erwähnt (I883, p. 27); doch ist er hier entsprechend der grösseren Form viel mächtiger entwickelt und viel schärfer, auch proximal und distal, von der iibrigen Subumbrella abgesetzt, so dass die i 6 Felder scharf begrenzt (Fig. 5 su pl) sind und sich auch an Schnitten besonders herausheben. Nach innen zeigen sich schwächere circuläre und radiäre Muskelfasern, nach aussen in den Tentakelradien stärkere Züge.

Beziiglich des Canalsystems kann ich die CLAUs'sche und Vantöffen'sche Darstellung gegen die MAYER'schen Angaben nur bestätigen. Der Lappencanal ist distal einheitlich; proximal aber erscheint eine trennende Spange.

Die Rhopalien sind von dem für Lausithoe bekannten Bau und von auffallender Grösse. Das Pigment des Ocellus ist ein Braun wie in den Gonaden, nur etwas intensiver.

Besondere Besprechung verdienen die Gonaden, die schon durch ihre kräftige Farbe an dem milchig durchsichtigen Schirm auffallen. An einigen Formolexemplaren ist die Farbe bis zu ziegelrot abgeblasst, an anderen aber noch braunrot oder carminrot erhalten, ähnlich der 
Agassiz'schen Skizze. Die Form der einzelnen Gonade ist nicht rund, sondern ausgesprochen viereckig oder schildförmig, indem die 4 Seiten unregelmässig ausgebuchtet sind, und die proximale Kante grösser ist wie die distale. Die Lage der Gonaden zu einander ist nicht in allen Exemplaren gleich. Manchmal liegen sie alle gleichweit auseinander. (Fig 6) wie es für Nausithoe von HAECKEL als charakteristisch angegeben wird, manchmal sind sie zu je zweien einander genähert, wie es bei Nausicaa sein soll. Diese Năherung kommt hier dadurch zu stande, dass die proximalen Enden je zweier Gonaden nach innen umbiegen und weiterwachsen, mitunter sich geradezu berïhren. Im extremsten Fall kommt dadurch in 4 Radien eine nieren-oder bohnenförmige, nach aussen offene Gonadenbildung zu stande, deren jede wieder durch einen proximalen Abschnitt zweigeteilt ist (Fig. 8). Dies Verhalten ist aber durchaus nicht constant, sondern bei verschiedenen Tieren, mitunter sogar in den verschiedenen Radien am selben Tier verschieden (Fig. 7); mitunter zeigt sich gar keine Annäherung (Fig. 6) [auch MLYER, der übrigens nur 2 Exemplare gehabt hat, erwähnt und zeichnet nichts davon], und zwischen den beiden Extremen finden sich alle Uebergänge. Die Annäherung wird durch das Wachstum beguinstigt, aber nicht bedingt; mitunter finden sich sehr grosse reife Gonaden vollständig gleich weit entfernt; mitunter zeigen schon kleine eine deutliche Annäherung. Also besteht auch hierin eine grosse Variabilität. Mit Sicherheit geht daraus hervor, dass die HaEckel'sche Gattung Nansicaa, für die die Annäherung je zweier Gonaden als charakteristisch angegeben wird, einzuziehen ist. Die betr. Art wird wohl unter $N$. punctata fallen.

Nun hat aber VAneörfen dasselbe Merkmal zur Trennung von N'ausithoe. nicht nur gegen Nausicar sondern gegen die ganzen Ephyropsiden (excl. Atolla-Formen) gebraucht (Palephyriden Haeckels), also gegen Ephyra, Pálephyra, Zoncphyra, die er mit Nansicar zusammen als eine Gattung, Paleplyya in erweitertem Sinn zusammen fasst. Seiner Ansicht nach haben die Palephyriden, nicht wie HAECKEL annahm, 4 interradiale Gonaden, sondern ebenfalls 8 Gonaden, die in den Perradien genähert sind, und er beschreibt dies Verhalten bei einer neuen Palephyra indica. Bei HaEckel's Nausicaa stossen jedoch die genäherten Gonaden im Interradius über den Gastralfilamenten zusammen und auch bei Palephyra und Zonchliyra werden die einheitlichen Gonaden bei HaEckel ausdrücklich als im Interradius liegend bezeichnet. VANHöfFEx hält es nun für nicht möglich, „dass innerhalb der Familie Ephyriden zwei ganz verschiedene Baupläne neben einander bestehen; so bleibe nur die Annahme übrig, dass HAeckeL, hier wie so oft, durch seine Phantasie irregeführt wurde". (I902, p. 32). Es wird von VANłörfex zu Gunsten seiner Ansicht das Verhalten von Atolla angefuihrt, wo nach HAEcker selbst die Gonaden perradial genähert sind, und der Vorschlag gemacht, die HAEckel'schen Figuren (IS79, Taf. XXVII) durch eine entsprechende Drehung um $45^{\circ}$ zu berichtigen.

Bei der hier vorliegenden Form kann ich jedoch auf das bestimmteste versichern, dass die Gonadenlage nicht wie bei Vaxhörfex's Palephyra sondern wie bei Haecker,'s Nazsicaa etc. beschaffen war. Wo eine Annäherung zweier Gonaden vorhanden war, lag äieselbe im In terradius; waren es 4 richtige (Hufeisen) Bohnen geworden, so lagen dieselben interradial, wie sich durch die perradialen Magenecken und die interradialen Filamentbüschel bei der durchsichtigen Meduse leicht feststellen liess. Es liegt mir natülich fern, den VANiöffex'schen Befund anzuzweifeln, umsoweniger als ich ja bei Atolla selbst die Annäherung in clen Perradien 
trotz interradialer Zusammengehörigkeit mehrfach erwähnt und abgebildet habe und auch bei Lincrges cine perradiale Annäherung beschreiben kann (s. u. p. 26). Es sind eben bei der VAniöfFen P'alctiy'a einerseits, bei den HAEckel'schen Formen und hier andrerseits, verschiedene Ausprïigungen vorhanden, ohne dass man deshalb von "ganz verschiedenen Bauplänen" zu reden brauchte. Es geht dies auch daraus hervor, dass laut VANï̈FFEn "das freie distale ${ }^{1}$ ) Ende sich..... nach innen einrollt, sodass sich schliesslich die benachbarten Gonaden im Perradius berihren". Hier aber, ebenso wie bei HAEckEL (s. Fig. auf seiner Taf. XXVII) ist es das proximale Ende, in dem die Gonaden zusammenkommen. Primär liegen ja bei allen Ephyropsiden und Discomedusen die Gonaden interradial, das Auseinanderrücken in die $S$ Adradien ist ein späiteres Verhalten, und durch weiteres Wachstum kann noch später in den Perradien eine Annäherung urspringlich nicht zusammengehöriger Gonadenpaare erfolgen. Das primäre noch genähert sein (interradial) bei den von HAECKEL und hier aufgefuihrten Formen ist zu unterscheiden von der secundären (perradialen) Annäherung bei VAnhöffex's Palcply'ra und bei Atolla. Gerade bei Atolla erfährt dies eine weitere Illustration durch ein grosses, nicht regelmässiges Exemplar aus clen Sammlungen des Fürsten von Monaco, über das ich an anderer Stelle berichte. Dasselbe zeigte nämlich anstatt $\delta$ nur $\uparrow$ Gonaden; eine aber von doppelter Grösse. Diese doppelte, oder besser gesagt einheitlich gebliebene Gonade lag aber interradial, trotz der grösseren Distanz in den Interradien, während die 6 anderen das typische Verhalten zeigten mit perradialer Näherung: Vian wird also nicht einfach Paleplayra als Ephyropside bezw. Nausithoide „mit länglichen Gonaden der Nousithoc mit rundlichen Gonaden" (VANHöfFex 1902,p. 51 ) gegenüberstellen können, sondern wird mehrere Gattungen, je nach der Lage und Gestalt der Gonaden zu unterscheiden haben. [Auch wenn man die TAviöffex'sche $P$. indica zu V'ausithoe stellen wïde, oder die vorliegende zu Palcphyra wären beide durch Form und Farbe der Gonaden, des Magens etc. noch unterschieden, ganz abgesehen von der Lag e der Gonaden]. Dass unter den HAECKEL'schen Ephyropsiden eine Reduction eintreten muss, kann CLAUS und VAvrö̈FEN gewiss zugegeben werden. Doch geht des letzteren Zusammenfassung aller in die eine Gattung P'alcphyra entschieden zu weit. Es ist, wie die erörterten Gonadenverhältnisse zeigen, jedenfalls eine grössere Formen-Mannigfaltigkeit in dieser interessanten Gruppe vorhanden. Es beweist dies neuerdings auch eine von A. Agassiz und MAYER beschriebene neue Zoncphyra corona (1902, P. I 57, Fig. 19), die sich ebenfalls nicht dem VANHöffex'schen Gattungsbegriff von Palcphyra einordnen wïrde. Dieselbe besitzt nur 4 Gonaden von Hufeisenform in den Interradien und zeigt ausserdem eine Vermehrung der Lappen von 16 auf 32, nicht aber der Tentakel und Rhopalien, sodass sich immer zwei Lappen zwischen Tentakel und Sinnesorgan befinden. Ferner existirt eine Bathylua solaris nov. gen., nov. sp. derselben Autoren, die bei ebenfalls 4 Gonaden, 8 Rhopalien, aber if Tentakel und entsprechende Randlappen aufweist (I902, Pl. I). Auch wenn man diese Verdoppelungen nicht als ein Genusmerkmal rechnet, und trotz der Vereinigung von Nausithoe mit Nauphanta, der Streichung von Nausicaa, blieben ausserdem unter den eigentlichen Ephyropsiden immer noch mindestens zwei, wenn nicht drei Genera für die bisherigen Formen. In das eine könnten die Arten mit 4 interradialen (Doppel)gonaden aufgenommen 
werden, in das andere die mit 8 perradial genäherten Gonaden, es könnte und um nicht zu viel Namensänderung zu veranlassen, für das erstere die Bezeichnung Zoncphyrra, für das zweite die Bezeichnung Palephyra beibehalten werden. Beide würden dann Nausithoc gegenüberstehen. Doch bedarf es zur endgiltigen Entscheidung noch weiteren Materials.

\section{Linergidae Haeckel i 879 .}

Die Linergidae wurden nicht nur als ganze Familie von HAECKEL creirt, sondern auch ihre einzelnen Vertreter von ihm zuerst beschrieben. Nach VAshöfres's Ansicht (1902, p. 50) „stehen sie den Nausithoïden weit näher als nach HaEckel's Darstellung zu vermuten ist", und werden wie diese als Unterfamilie zur Familie der Ephyropsidae bei den Coronaten eingeordnet. VAxröFFEN betont die Aehnlichkeit der Rhopalien, der Schirmbildung und der Pedalien mit den Nausithö̈den, und hält auch des Vorhandensein eines Ringcanals, trotzdem es HAEckel anders zeichnet, für wahrscheinlich, wenn er es auch nicht an eigenen Exemplaren belegen konnte. Um so angenehmer ist es mir, im Sibogamaterial einige allerdings jugendliche, aber sehr gut conservirte Linergiden zu besitzen, die die Entscheidung über die von VANHöffen angeregten Punkte ermöglichen. Es lässt sich in der That eine Communication zweier benachbarter Taschenzipfel constatieren, und ferner ein deutliches perradiales Zusammenliegen von je zwei Gonaden. VANıörfex ist zur Streichung zweier der HAEckEL'schen Gattungen geneigt, weil er wie bei den Nausithoïden, nur eine perradiale Gonadenannähernng für möglich und die HAECKEL'schen Angaben und Abbildungen von interradialen Gonaden für falsch hält (s. O. P. 2I). Er will deshalb nur "Linantha (incl. Linerges und Liniscus), bei der die \& Gonaden mehr oder weniger länglich und paarweise gruppiert erscheinen, und Limuche, die 8 kugelige Gonaden mit ziemlich gleichen Abständen besitzt", unterscheiden ähnlich wie Palcphyra und Nausithoe sich laut seiner Darstellung unter den Nausithö̈den verhalten. Dort aber habe ich gezeigt, dass es auch interradial genäherte oder event. sogar einfache interradiale Gonaden geben könne, (s. O. P. 2I); ich glaube daher, dass wir mit der Streichung auch hier nicht zu weit gehen und ebenfalls mindestens drei Möglichkeiten im Auge halten müssen. Hier habe ich allerdings selbst, anders wie bei Nausithoiden, einen Fall perradialer Gonadennäherung gefunden; ich halte aber clie interradiale Lage dennoch nach Analogie für möglich. Um nun den Sinn der HAEckel'schen Gattungen, im Falle solche Medusen wie sie HAECKEL auf Taf. XXIX zeichnet, wieder gefunderi werden, nicht zweimal ändern zu müssen, würde ich für letztere den Namen Linantha beibehalten, für Formen mit 8 gleichmässig verteilten Gonaden Linuche, und für solche, wie hier, mit länglichen, sich perradial nähernden Gonaden, Lincrges, um so mehr als ich in der vorliegenden eine der HAEckel'schen Linerges-Species zu erkennen glaube. Auch die von AG.1ssiz und MAYER gefundene Linerges-Art wird, wenn auch nicht spezifisch, so doch generisch hierhergehören; sie besitzt vier halbmondförmige Gonaden, die Convexität proximal gelagert, aber jeder der Halbmunde ist durch „ein medianes Septum" zweigeteilt, und es entstehen dadurch, wie die Figur 33 ( 1899, Pl. 10) zeigt, \& paarig liegende Gonaden, wie hier. Auch die HAEckel'schen 
Iiguren von Linerges zeigen, im Gegensatz zu seiner Linantha-Figur, die Trennungslinie der (allerdings interradialen) Gonaden noch recht deutlich.

Die Arten, wie sie bei HAECKEL aufgeführt sind, werden jedenfalls eine grössere Reduction erleiden müssen; insbesondere glaube ich, dass die Liniscus-Arten mit Linerges-Arten als nur zeitlich verschiedene Stadien zusammenfallen. Die definitive Entscheidung kann erst an weiterem Material erfolgen. Die Zahl der Subumbrellar-Säckchen ist für die Artunterscheidung kaum zu verwenden, da sie im Lauf der Entwicklung steigt, so das zuerst nur eine circuläre Reihe, dann 2, 3 etc. vorhanden sind. Form und Grösse der Lappen und Tentakeln, die Verzweigung der Canäle, scheinen hierfür bessere Hinweise zu liefern, vielleicht auch die Farbe, die allerdings erst bei den $2 \mathrm{nach}$ HAEckEL noch einmal beschriebenen Formen bekannt ist, bei der von Agassiz und Mayer beschriebenen $L$. aquila und bei der hier vorliegenden $L$. draco. Die ersteren Autoren erwähnen ausdriicklich, dass sie in Ermangelung einer Figur und genauen Beschreibung nicht sicher sind, auch die HAECKEL'sche Form aquila vor sich gehabt zu haben; dasselbe gilt für draco hier; aber es wird dadurch sehr wahrscheinlich, dass sich meine und die Agsssiz'sche Form gerade durch Merkmale unterscheiden, die L. draco von L. aquila trennen. Bei draco sind die Tentakel auffallend kurz, die Lappen fast nierenförmig, halb so lang als breit; ferner die Lappencanäle zahlreich, verbogen und ausgebuchtet (s. Fig. I u. 2); bei aquila sind dieselben breit, handförmig (s. I \$99, Taf. X). Die Säckchen-Zahl (bei AGAssiz und MAYER 52 in 5 Reihen) ist hier nur 16 in einer Reihe; doch handelt es sich um jugendliche Exemplare.

\section{Linerges Haeckel i 879 .}

Sens. emend. Maas.

Linergide mit 8, in den Perradien paarweise genäherten Gonaden.

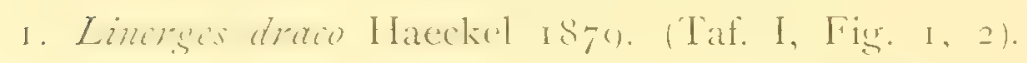

Stat. 289. 3 Exemplare von $\mathrm{I}-\mathrm{I}, 2 \mathrm{~cm}$. Durchmesser und $\mathrm{O}, \mathrm{S}-\mathrm{I} \mathrm{cm}$. Höhe.

Der Schirm ist auffallend hoch gewölbt, die Seitenwände fallen, wie auch HAEckel und MAYrer bei ihren Linergiden hervorheben, fast senkrecht ab, der Scheitel ist flach. Die Gallerte ist iiberall gleichmässig und nur sehr duinn, blattartig entwickelt. Dadurch, wie durch die hohe Wölbung und geringe Randkerbung erhält der Habitus etwas trachynemidenartiges, wie bei Rhopalonema. Der grössere distale Teil der Exumbrella ist durch I 6 scharf eingegrabene Furchen in 16 Felder geteilt, die den $S$ Rhopalar- und 8 Tentakularpedalien entsprechen. Eine centrale Schirmkuppel ist an den vorliegenden Stïcken nicht wahrzunehmen; man müsste denn das furchenfreie Mittelstiuck so nennen. Auch eine trennende Ringfurche ist nicht vorhanden, sondern Pedalien und Furchen verlaufen allmählich nach der Schirmmitte zu. Die ganze Exumbrella ist mit ovalen Nesselwarzen bis zum Rande dicht gespicht (s. Fig. 2 nw).

Das Magenrohr hängt in die Glockenhöhlung ungefähr zu deren Hälfte herunter und ist von der typischen Bildung der Ephyropsiden. Es zeigt 4 perradiale Kanten, 4 interradiale Flächen, die durch je 2 adradiale Gallertstäbe etwas verstärkt sind. Diese 4 interradialen Flächen 
springen auch an der Mundöfnung als gekerbte Lappen vor, so dass die eigentliche Mundöffnung sich auf ein perradiales Kreuz verengt. Auch die perradialen Ecken des Mundes zeigen eine Verlängerung (Fig. I). An der Basis stehen die 4 perradialen Ecken des Magenrolirs durch besondere, stuitzende Gallertspangen mit der Subumbrellargallerte in Verbindung, ähnlich wie ich es für Atolla beschrieben (1897, p. 68). Diese Spangen sind es, die durch Gabelung die erwähnten Verstärkungen der interradialen Magenwände liefern, ebenfalls wie bei Atolla ( 1 sig-, Taf. XiI, Fig. 3).

Der Centralmagen setzt sich noch eine Strecke weit einheitlich (abgesehen von den 4 Verwachsungen) auf der Decke der Subumbrella fort, um sich dann in I 6 Taschen zu spalten, die in den Radien der Rhopalien und Tentakel liegen und durch Cathammen getrennt werden, deren Lage genau den 6 Furchen der Exumbrella entspricht. Von jeder Tasche springt gegen die Subumbrella eine Aussackung vor, von ovaler bläschenförmiger Gestalt. Hier kann man 8 kleinere rundliche Säckchen im Radius der Rhopalien, und 8 grössere eiförmige im Radius der Tentakeln unterscheiden, die alle zusammen in einer, mehr proximalen Circulärreihe liegen; weitere circuläre Reihen, mit doppelt oder halb oder viertel soviel Bläschen, sind hier noch nicht gebildet. Was die Bedeutung dieser Säckchen ist, darüber kann ich nichts bestimmtes aussagen; nur möchte ich mich gegen HaEckEL's Annahme aussprechen, dass es Hoden seien, und die Linergiden darnach Hermaphroditen. Das Entoderm der Säckchen zeigt absolut keine Aenderung gegenüber dem übrigen Entoderm der Taschen, zur Zeit, wo die Gonaden doch schon deutlich differenziert sind. Die histologische Verschiedenheit der Säckchen von der übrigen Subumbrella rührt, wie bei den Subumbrellarplatten der Nausithoiden, von einer Verstärkung des Ectoderms.

Der weitere Verlauf des Canalsystems, ehe es die Lappen erreicht, gestaltet sich durch Cathammenstreifen etwas unregelmässig; die verlöteten Stellen bleiben indessen immer ziemlich schmale Streifen, gegenüber dem wegsamen Canalsystem (Fig. 2). Ein Anastomosennetz mit kleinen Canälen und grossen Verwachsungsfeldern, wie es HAEckel für Linerges mercurius zeichnet (I879 Taf. XXIX, fig. 6), ist hier nicht, oder noch nicht gebildet. An den Lappen zeigt das Canalsystem die von VAnHöffen vermutete Bildung. Die 16 adradialen Septen oder Cathammen, setzen sich (im Gegensatz zur HAECKEL'schen nicht zutreffenden Abbildung 1. c. Fig. 3, 5, 6) nicht bis zum Ende der Randlappen fort, sondern hören ungefähr in der Höhe der Lappeneinschnitte mit einer knopfartigen Verbreitung auf. Dadurch kommt eine bogenförmige Communication zweier Nachbartaschen am Lappenrand zu stande, ngewissermassen ein Ringcanal" (Vanh. I902), den VANHÖFFEN mit Recht dem Ringcanal der Ephyropsiden vergleicht. Allerdings ist er hier proximal durch Cathammen verengert und distal durch Verästelung verändert; aber es ist doch morphologisch dieselbe Bildung. Diese Verästelungen sind hier von ganz charakteristischer Gestalt, verbogen und wieder secundär verästelt, so dass man schon an diesem jugendlichen Stadium von $10 \mathrm{~mm}$. Durchmesser von fast 30 blinden Terminalästen reden kann, wie es HaEckel für $L$. draco angiebt. Im Gegensatz dazu zeichnet Marer bei $L$. aquila einen Lappencanal wie eine Hand, mit nur $6-S$ unverzweigten Aesten, wie Finger, auf einem Grössenstadium von I $6 \mathrm{~mm}$. Breite, I $3 \mathrm{~mm}$. Höhe. Durch die lichte schilf-grüne Farbe, die allen peripheren Entodermteilen, auch den Subumbrellarsäckchen hier eigen ist (Fig. I u. 2 ent u. ri), heben sich die Canäle sehr gut von der Lappengallerte ab. 
Die Randlappen selbst sind hier, ebenfalls im Gegensatz zu L. aquila, breiter als lang; an der Basis sind sie eingebuchtet und dann wieder verbreitert, so dass man sie mit Hafcres. "fast nierenförmig" nennen kann. Der Nesselwarzenbesatz ist auf ihrer Exumbrellarseite sehr reich entwickelt, schneidet aber an ihrem Subumbrellarrand ab. Die Randlinie selbst erscheint dadurch an jedem Lappen noch einmal in lauter kleine Bogenläppchen ausgeschnitten (Fig. 2), wie es ähnlich auch HAECKEL für L. morcurius zeichnet.

Die II uskulatur der Subumbrella zeigt einen Ringmuskel, der sich aber in Stärke nicht mit dem von Atolla vergleichen lässt und auch hinter dem von Nausithoe beträchtlich zuruickbleibt. Er zerfällt nicht in I 6 Tafeln, sondern ist einheitlich; man sieht die Fasern ohne Unterbrechung oder Verdiunnung auch ỉber die i 6 radialen Lappenspangen ziehen, im Gegensatz zu HAECTEL's Angaben. Nach einwärts zeigen sich in diesen i 6 Radien starke radiäre Muskelzüge; ebenso strahlen einige schwächere Radiärzüge nach auswärts aus. Auf dem Circulärmuskel liegt auch hier ein stärkerer ectodermaler Nesselbelag. Derselbe zeigt nicht nur in den 16 Radien, sondern auch dazwischen, radiäre Unterbrechungslinien, wodurch eine Cathammenbildung im Entoderm vorgetäuscht werden könnte.

Die Tentakel sind auffallend kurz, kürzer wie die Lappen selbst, und zugespitzt. Thre Basis lässt sich bis in die Schirmgallerte hinein verfolgen. Man sieht eine Zapfenbildung wie bei Nousithoe. Das axiale Entoderm ist an der Basis hohl und ordnet sich dann zu einem soliden Zellstrang an.

Die Rhopalien sind von breiter gedrungerer Form und gleichen in ihrem Bau durchaus den von Nausithoe bekannten Kolben.

Die Gonaden sind hier noch nicht ganz entwickelt, zeigen aber die erwähnte charakteristische Näherung im Perradius und gleichen dadurch 4 unterbrochenen Halbmonden. Ihre weitere Ausbildung, die zu einer besonderen Lappung führen soll, konnte hier nicht beobachtet werden.

Bei der Aehnlichkeit, die die Linergiden mit den Cyaneiden zeigen (auch bei diesen kommen verästelte Lappencanäle und in die Subumbrella ausgestiilpte Entodermteile vor) ist eine nähere systematische, vielleicht sogar ontogenetische Zusammengehörigkeit nicht ausgeschlossen. MIan könnte sich eine Dissogonie vorstellen, bei der aus einer geschlechtsreifen Linergide nach Ablaichung durch Metamorphose eine Cyaneide wieder olne Gonaden wuirde.

\section{III. l)iscoplorat. \\ A. Semaeostomata.}

\section{Aurelia.}

I. Aurclia aurita Linn. var. colpota. (Taf. I, Fig. 9). 
$\begin{array}{lrrr}\text { Stat. I40. } & 3 \text { Exemplare von etwa } 12 \mathrm{~cm} \text {. Durchmesser. } \\ \text { Stat. I69. } & 2 \text { Exemplare von } & 10 \mathrm{~cm} \text {. } \\ \text { Stat. 209. } & 8 \text { Exemplare von } & 6-64 \mathrm{~cm} . \\ \text { Buton-Strasse. } 6 & \text { Exemplare von } & 8-12 \mathrm{~cm} .\end{array}$

Die Untersuchung dieser zahlreichen Exemplare von 4 Localitäten aus dem Indischen Ocean bestätigt die von VAnHöffen hervorgehobene grosse Aehnlichkeit der dortigen Form mit der bekannten Aurclia aurita und die Möglichkeit der spezifischen Vereinigung; doch scheint es mir nicht ganz sicher, ob man damit so weit gehen kann wie VANHöFfEx, welcher zu aurita 8 der beschriebenen Species zieht, sodass ausser dieser nur noch eine arktische Art verbleibt. Mir erscheinen nach den vorliegenden Beschreibungen vorderhand 3 Arten abgrenzbar und innerhalb der Species am ita selbst einige Varietäten deutlich erkennbar. Gerade die Verzweigung des Canalsystems, die noch am besten etwelche Unterschiede begründet und Uebergänge vermitteln soll, ist an allen vorliegenden Exemplaren von ganz überraschender Uebereinstimmung. Abweichungen kommen nur in Verbindung von kleinen Unregelmässigkeiten, wirklichen Abnormitäten, Vermehrung und Verminderung der Antimeren vor, wie sie bei Aurelia aurita nicht selten sind. Als typisch für das Canalsystem kann hier gelten: I) die $4+4$ Rhopalarcanäle laufen durchaus unverzweigt zum Schirmrand, ebenso die 8 Radialcanäle; 2) die zwischenliegenden Canäle verzweigen sich dichotomisch in symmetrischer Weise derart, dass immer ein Ast nach Abgabe des andern die ursprüngliche Richtung beibehält (Fig. 9). 3) Anastomosen kommen nicht vor, sondern die Canäle gehen annähernd parallel zum Schirmrand. Wichtig ist die Art und Weise, wie sich die verzweigten Canäle an ihrem Ursprung zu den 8 adradialen und $\&$ rhopalaren verhalten. Die 8 adradialen sind stets durchaus gesondert, dagegen weisen die 8 rhopalaren Canäle Beziehungen zu den verzweigten Zwischencanälen auf. Man sieht an den interradialen häufig, dass die Zwischencanäle von deren Basis und nicht selbstständig aus der Magenperipherie entspringen; an den perradialen ist das weniger markant; doch zeigt sich auch hier öfters noch ein Zusammenhang (Fig. 9). Es ist dies Verhalten ein Zwischenstadium zwischen dem typischen Verhalten von aurita (s. CLAus i 878 , Taf. V) wo nach HAECKEL'scher Zählweise 3 Canalwurzeln in jeden Genitalsinus vorhanden sind, und dem typischen Verhalten von colpota, wo 5 solcher Canalwurzeln gezählt werden. Dies kommt dadurch zu stande, dass die eradialen verzweigten Canäle selbstständig wurden, und scheint hier dás endgiltige Verhalten. Durch fortschreitende Selbstständigkeit der einzelnen Gabeläste kömnte man sich dann das Vorkommen von 7 Canalwurzeln erklären, wie es für A. flavidula charakteristisch sein soll. Die schöne Figur von L. Agassiz (1860, Pl. VII) zeigt solche Uebergänge in der That an. Doch möchte ich flavidula im Sinne von Agassiz noch einstweilen als Varietät, wie auch colpota gelten lassen. Die MAYER'sche vitiana stimmt aber vollkommen mit colpota überein und ist auch nicht als Varietät abzutrennen, umsoweniger als MAYER, wie stcts, nicht einmal eine Differentialdiagnose trotz schon vorhandener II Arten giebt. Ein Unterschied besteht nur insofern, als bei MAYER die Tentakeln gelb, die Gonaden violett, bei der BRANDT'schen Abbildung von colpota, beide rötlich sind. Die Farbe ist aber bei Aurclia aurita sehr variabel und spielt (s. HAECKeL p. 554) vom Violetten im Fleischrote. Die hier vorliegenden Stïcke haben rosa (fleischfarbene) Gonaden und heliere ins Gelbliche spielende Tentakeln. 
Einzelne Unregelmässigkeiten am Schirmrand sind nicht selten; alsdann können sich auch die parallelen Canäle einandern nähern und Anastomosen eingehen, was im regelmässigen Fall niemals eintrifft. Eine wirkliche Teilung der Lappen zwischen 2 Rhopalien findet nicht statt; eine leichte adradiale Einkerbung findet sich wie bei allen Aurelien, so auch hier. Die Mundarme sind in Länge etwas variabel, doch selten erheblich kiurzer oder länger als der Schirmradius. Der Zustand der Gonaden erwies die meisten Tiere als nahezu geschlechtsreif.

\section{Cyanea.}

I. Cyanea fermiginca Eschsch. I 829 .

Cyanca formginea Goette I886.

Stat. 31S. I Exemplar von fast $50 \mathrm{~cm}$. Schirmdurchmesser.

Die Systematik der Cyanea-Arten sowie der verwandten Gattung Desmoncma würde eine gründliche Revision erfordern. Letzteres von L. Agassiz aufgestelltes Genus wurde von diesem Autor als Pelagide aufgefasst, von HAECKEL jedoch zu den Cyaneiden gestellt und soll sich von der Gattung Cyanea nur dadurch unterscheiden, dass die Tentakel in einer Reihe im Bündel stehen, während sich bei Cyanea jedes Bündel aus mehreren Reihen zusammensetzt. Haeckel selbst beschrieb eine Desmonema Amasethe von der West-Küste Suid-Africa's, die abgesehen von dem obigen Unterschied durchaus Cy'anca gleicht. Die eigentiumliche Einteilung des Schirmrandes, die Muskelfelder, die Armgardinen etc. zeigen das typische Verhalten, und auch die „in einer Reihe nebeneinander" stehenden Tentakel sind eigentlich nicht in einer Reihe nebeneinander geordnet, sondern stehen in Hufeisenform, wie bei Cyanca, nur dass dieses Hufeisen in der Breite immer nur einen Tentakel begreift (Taf. XXX), bei Cyanea 3 oder 4. Dies ist gewiss ein sehr relatives Merkmal; bei seiner Cyanca Annaskala bemerkt Lendexfeld I882, p. 470 „während bei den nördlichen Cyaneen die Tentakelansätze auch am centrifugalen Ende mehrreihig angeordnet sind, erscheinen dieselben bei C. Amaskala der Peripherie zu in einer einzigen Reihe angeordnet. Diese Eigentümlichkeit stempelt unserer Meduse zu einem Uebergangsgliede zwischen den Gattungen Cyanca und Desmonema". Bei C. arctica, die ich aus eigener Anschauung in Norwegen in sehr verschieden grossen Exemplaren kennen gelernt habe, wird das Desmonemastadium ontogenetisch durchgemacht; also besitzt auch Cyanea zuerst nur ein einfaches Hufeisen. Bei der geringere Grösse der HAEckeL'schen Dcsmoncma einerseits und der bekannten riesigen Ausprägung der Cyaneen andrerseits, die zu den grössten Medusen zählen, halte ich D. Annasethe nur für eine Jugendform von Cyanea, ebenso wie die von A. Agassiz und Mayer (isg\&, pl. 1) abgebildete D. rosea n. sp. Die andern beiden, dem A Gussiz'schen Begriff der Gattung entsprechenden Desmonemaarten sind kaum wieder erkennbar, und das Genus ist daher meiner Ansicht nach aufzulösen.

Das Verhältnis der Cyaneaarten untereinander bedarf ebenfalls noch der Klärung. Die C. amaskala Lendexpeld's ist von HaAcre als C. Miullorianthe wieder beschrieben worden. HAACKE selbst erwähnt die benachbarten Fundorte und den „engsten" Anschluss an LENDENFELD's Art. Der einzige Unterschied ist der, dass bei letzterer die Bursalsepten ganz gerade, 
bei seiner Art eigentümlich geschwungen sind, und eine geringe Farbenverstärkung im Ton der Mundarme (!). MAYER erwähnt bei seiner, ebenfalls australischen D. rosea die Aehnlichkeit mit beiden genannten Arten, und es kann nach Vergleich der betreffenden Beschreibungen und Abbildungen wohl kaum ein Zweifel sein, dass sie einer einzigen Art angehören und beide nicht ganz erwachsene Stadien darstellen, ebenso wie D. Amascthe. Weniger sicher ist festzustellen, wie sie sich zu andern Arten verhalten, spez. auch zu C. formginea. Als solche hat Goette Medusen von Singapore bestimmt, und mit dieser Form stimmt nach der alten Abbildung und Beschreibung (I 829 , p. 70, Taf. V) auch das vorliegende Exemplar am meisten überein; die Eschscholtz'sche Art stammt allerdings aus dem nördlichen Pacific, und da sich auch eine sehr grosse Aehnlichkeit meines Exemplars mit der australischen Form ergiebt, so ist die Entscheidung nicht ganz sicher. Möglicherweise fällt die von LENDENFELd etc. beschriebene Form als Jugendstadium unter die Eschscholtz'sche. Bei dem Mangel an Material - es steht mir nur dieses eine erwachsene und zu schonende Exemplar zu Gebote - kann ich auf alle diese Fragen nur hinweisen und muss die Entscheidung späteren Untersuchern überlassen.

\section{Pelagia.}

I. Pelagia panopyra Pér. \& Les. I809.

(Pelagia pospliora? Haeckel) I879.

\begin{tabular}{|c|c|c|c|c|c|c|c|}
\hline $\begin{array}{l}\text { Stat. } 53 . \\
\text { Stat. I06. }\end{array}$ & $\begin{array}{l}\text { I } \\
2 \text { junge }\end{array}$ & $\begin{array}{l}\text { Exemplar } \\
\text { Exemplare }\end{array}$ & & $\begin{array}{l}\text { von } \\
\text { von }\end{array}$ & nicht & $\begin{array}{l}4 \mathrm{~cm} . \\
\mathrm{rcm} .\end{array}$ & $\begin{array}{l}\text { Durchmesser. } \\
\text { Durchmesser. }\end{array}$ \\
\hline Stat. Iog. & 2 & $\eta$ & & & $n$ & $n \quad$, & $"$ \\
\hline Stat. 14I. & I & n & & & & & \\
\hline tat. I 48. & I & , & & & & & \\
\hline tat. $214 \cdot(23 / x)$ sehr & zahlr. & n & (Schwarm) & " & $1 / 2-4$ & $-4 \mathrm{~cm}$. & " \\
\hline itat. $21+4 \cdot\left({ }^{21} \mathrm{~s}\right)$, & . & " & " & & - & $"$ &. \\
\hline Stat. 256 & 2 & $"$ & & , & & I, & , \\
\hline at. 284 . & I & , & & $\pi$ & & $\eta \quad \pi$ & $n$ \\
\hline
\end{tabular}

Von den zahlreichen bisher beschriebenen Pelagia-Arten (bei HAEckel werden Io angeführt, VANHöFfEN hat dieselben um 3 vermehrt (I888), dazu kommt noch eine weitere von A. G. MAYER (1902)) werden sich gewiss mehrere vereinigen lassen, wie schon eine Vergleichung der Beschreibungen in der Litteratur ergiebt. Goerte will $P$. flaveola und denticulata zusammenfassen (1886, p. 834), VANHöFfEN, der friher selbst eine Aufstellung von unterscheidenden Merkmalen versucht und dabei besonderen Wert auf die Nesselwarzen gelegt hat, vereinigt neuerdings phosphora und panopyra (1902, p. 37) und giebt an, dass zwischen diesen beiden und $P$. noctiluca Uebergänge in der Faltung der Nesselwarzen bestehen. Die hier vorliegenden Stiicke zeigen sämmtliche, für $P$. panopyra als charakteristisch angefuhrten Merkmale, insbesondere das lange Magenrohr, die grossen Mundfahnen, die kleinen rundovalen Nesselwarzen, die quadratischen Randlappen etc. Grosse Aehnlichkeit zeigen sie allerdings auch mit einer Art, die mir in mehreren Exemplaren aus dem subtropischen atlantischen Ocean zum Vergleich dient, die jedoch ein im Verhältnis weit kürzeres Mundrohr und etwas andere rundlichere Nesselwarzen zeigt, und die ich vorläufig als $P$. perla bestimmt habe. Ich stimme VANröffex darin bei, dass 
., eine Revision der Pelagia-Arten einstweilen aufgeschoben werden muss, bis genügend umfangreiches Material aus allen Meeren vorliegt" (I902, p. 37); für die vorliegenden Stiucke behalte ich den Namen der pacifischen $P$. panopyra bei; sollte sich, wie VANHöFfex annimmt, eine Identität mit der atlantischen $P$. phosphora herausstellen, so hat letzterer Name zu fallen, der erst I 879 von HAECKEL gegeben wurde, während $P$. panopyra mit guter Abbildung und deutlicher Diagnose schon i 829 von Eschscholtz aufgestellt worden ist.

Die Pelagien sind, wie TANmöffen hervorhebt, „die einzige Acraspeden Gattung die unabhängig vom Land.... in allen ihren Entwicklungsstadien der Hochsee angehört" ${ }^{1}$ ) (p. 34). Die Schwarmbildung wirc nach seiner plausiblen Ansicht durch das Zusammentreffen zweier Strömungen hervorgerufen, wie er an einigen Beispielen darlegt. Im malayischen Archipel herrschen, wie WEBER in seiner vorläufigen Mitteilung betont, besondere Verhältnisse. Durch die Inselverteilung einerseits, die Strömungen andrerseits, kommen Hochsee- und Küstenplankton zusammen vor, und das Plankton kann local zu echten Tierstrassen angehäuft sein, während es anderwärts nur sparsam auftritt (1900, p. 6).

Die meisten der Exemplare, besonders aus dem Schwarm Stat. 2 I 4 etc, sind Jugendstadien, von solchen mit nur 4 Tentakeln, die sich an die Ephyra anschliessen bis zu solchen von $\mathrm{I}^{1} / 2 \mathrm{~cm}$. Durchmesser, die bereits die Anlage der Gonaden zeigen; aber auch die grössten hier vorliegenden Stücke scheinen mir noch nicht völlig ausgewachsen zu sein. Der Schirmrand behält an allen seine furr die Gattung charakteristische Einteilung. Interessant ist, dass sich an einem grossen Exemplar ein Cirriped (wohl Lepadide) im Metacyprisstadium angesetzt hatte, meines Wissens der erste dieserart bekannte Fall. Sonst diirfte die Grösse und Schwimmfähigkeit einer Meduse einem Cirripeden zum Ansitzen nicht genügen. Die Meduse war noch durchaus normal (etwa $3 \mathrm{~cm}$. im Durchmesser, der Cirriped etwa $0,6 \mathrm{~mm}$.). Wie sich das Verhältnis beim Weiterwachsen gestaltet hätte ist schwer vorzustellen.

\section{B. RuIZOSTONEA.}

a) Rh. dichotoma.

Die VAnnöffex'sche Gruppe der Rh. dichotoma entspricht nahezu vollkommen der CLAUS'schen Familie der Cepheidae. Bei dem HAECrEL'schen Haupteinteilungsprinzip (getrennter oder einheitlicher Subgenitahraum) wurde die CLAUs'sche Familie gänzlich aufgelöst; die Gattungen Cephac und Polyrhiza als Unterfamilie Polyrhizidae zur HaEckel'schen Familie der Toreumidae, die Gattungen Cotylorhisa und Stylorhiza als Unterfamilie Stylorhizidae zu den Versuriden gestellt, Phyllorthisa bei den Pilemiden untergebracht. VANnörfex hat unter Geltendmachen der Claus'schen Bedenken, dass die Structur des Subgenitalraums sich im Lauf der Entwicklung von der getrennten zur einheitlichen Form verändere, und dass diese Aenderung, wie HAECKEL selbst

1) Es ist dies durch die Entwickelungsgeschichte verständlich. Bei Pelagia ist das festsitzende Scyphistomastadium unterdrückt und die l'lanula seht direkt in die Ephyra iiber. 
angiebt, sogar unter Umständen ausbleiben könne, und mit Hinzufügung neuer Untersuchungen, die HAEckEl'sche Trennung wieder aufgehoben und die CLaUs'schen Gattungen, nach Ausschluss von Phyllorhiza, die er mit Recht als triptere einordnet, unter seiner Gruppe dichotoma vereinigt. L. S. Schultze ( 1898 , p. 46I) hat demgegenüber wieder den HaEckes'schen Standpunkt zu rechtfertigen versucht. Er betont, dass in keinem einzelnen Fall ein Autor im Zweifel geblieben wäre, welche Form der Subgenitalbildung vorliegt, und dass auch CLAus und VAXIIÖFFEX selbst das Merkmal mitunter für Art- oder Gattungsdiagnose verwenden. Diese Verteidigung ist gewiss nicht stichhaltig; denn wenn auf Grund der Subgenitalstructur Gattungen die nach allen ïbrigen Merkmalen offenbar nahe zusammen gehören, wie hier, oder wie in andern Fällen (Cramborhiza und Lychnorhiza), die VANHöFFEN beleuchtet (1889, p. 29) auseinander gerissen wïrden, so darf dieser einzelne Unterschied eben einfach nicht zur Kennzeichnung ganzer grosser Gruppen verwendet werden, auch wenn er im speziellen Fall einmal mit zur Gattungsdiagnose dienen kann.

Auch noch andere Thatsache helfen dies hier bestätigen. Die HAEckel'sche Unterfamilie Polyrhizidae hat gabelteilige Mundarme, ebenso wie die von ihm so entfernt untergebrachte Unterfamilie der Stylorhizidae, und unterscheidet sich von dieser, abgesehen von dem problematischen Subgenitalraum, durch nichts als wie dadurch, dass bei der ersteren żwischen den Saugmündchen lange Peitschenfilamente, bei der letzteren grosse gestielte Saugnäpfe zur Ausbildung kommen. Nun hat aber L. S. Schultze selbst zwei neue „Polyrhiziden"-Ga.tungen, (Netrostoma und Halipetasus) auf Grund des Semonschen-Materials gemacht, die jedoch keine Filamente besitzen, sondern Anhänge ganz ähnlich wie Cotylorfiza und die "Stylorhiziden" überhaupt. Nur weil sie aber getrennte Subgenitalräume zeigen, werden sie bei den Polyrhiziden untergebracht und „erfordern eine Erweiterung der Diagnose der Subfamilie" (I $\$ 9 \delta$, p. 456), während sie sich in die Claus'sche und VANHÖFFEx'sche Einteilung zwanglos gefuigt und vielleicht nicht einmal die Aufstellung neuer Gattungen nötig gemacht hätten. Auch mir liegen hier Formen mit gabelteiligen Mundarmen vor, ebenfalls ohne Peitschenfilamente, aber mit eigentümlichen spindelförmigen Anhängen iuberall an den Armen, und auch in andern Details den Schultze'schen neuen Gattungen sehr ähnlich. Jedoch ist bei den einzelnen Individuen der Subgenitalraum sehr verschieden gestaltet und zeigt verschiedene Abstufungen von 4 völlig getrennten Höhlen bis zu einem einheitlichen, nur durch Septen geteilten Stadium (s. u. p. 35). Es bedarf also wohl keiner weiteren Rechtfertigung alle diese Formen in einer Gruppe zu vereinigen. Da bei den Schultze'schen Gattungen noch weitere unterscheidenden Merkmale dazukommen, da ferner auch von A. Agassiz und MAYER eine neue hiergehörige Form, unter dem Namen Cephea dumokuroa beschrieben wurde (IS99, PI. I I und I 2), jedoch bei allen eine Differential-diagnose fehlt, so empfiehlt es sich, die bisherigen Gattungen zunächst mit den wichtigsten Merkmalen zusammenzustellen.

Cephaca (Pér. u. Les.). Mundarme einfach gabelteilig; zwischen den Saugkrausen zahlreiche lange Peitschenfilamente; im Canalsystem 8 gesonderte Rhopalarcanäle, dazwischen 32 und mehr schwächere Radiärcanäle. Exumbrella mit Höckern; Schirmkranz und Kuppel gesondert. 
Polyrhiza (L. Ag.). Mundarme wiederholt gabelteilig; zwischen den Saughrausen zahlreiche Peitschenfilamente, 8 gesonderte Rhopalar-, zahlreiche, (32 u. mehr), andere Radiärcanäle. Exumbrella ohne Höcker, jedoch mit Radialfurchen.

Cotylorliza (L. Ag.). Mundarme einfach gabelteilig, Gabeläste gefiedert, zwischen den Saug. krausen keine Peitschenfilamente, dagegen gestielte Saugnäpfe. Von jedem der 4 Magenlappen etwa 8 Radiärcanäle mit gleichmässiger, reicher Verästelung ausgehend. Exumbrella glatt.

Stylorhiza (Haeckel). Mundarme wiederholt gabelteilig; zwischen den Saugkrausen langgestielte Saugnäpfe, besonders starke Rhopalarcanäle; dazwischen ein engmaschiges Gefïssnetz. Exumbrella ohne Auszeichnung.

Nctrostoma (L. S. Schultze). Mundarme wiederholt gabelteilig; lateral stark comprimirt; keine Filamente zwischen den Saugkrausen; in der Mitte der Armscheibe einzelne spindelförmige Anhänge. Im Canalsystem 8 gesonderte Rhopalarcanäle, dazwischen 24 andere Canäle mit zahlreichen seitlichen Blindästen. Exumbrella mit Höckern. Schirmkranz und Kuppel gesondert.

Halipctasus (L. S. Schultze). Mundarme wiederholt gabelteilig; dorso-ventral comprimirt. Saugkrausen ohne Filamente, aber mit zahlreichen kleinen Anhängen. 32 fast gleichmässig verästelte Radiärcanäle. Exumbrella ohne Höcker, nur mit zerstreuten, kleinen Körnern. Schirmkranz und Kuppel nicht gesondert.

ANm. Während des Druckes erschien: K. Kishinovye. Some new Scyphomedusae from Japan. Journ. Coll. Sc. Tokyo. vol. XVII. Auch dieser Autor sieht sich genötigt, die HAECKEL'sche Familiendiagnose zu erweitern, und fügt 2 neue Gattungen, Microstylus und Pcrirhiza hinzu. Deren erste fällt vielleicht mit Netrostoma in modifizierter Diagnose zusammen.

Die hier vorliegenden Stücke von drei Localitäten passen in keine der aufgeführten Gattungen vollkommen, wenn sie sich auch, vom Genitalporticus abgesehen, am nächsten an die beiden letzten anschliessen. Nach L. S. Scinulzz's Vorgang wäre also einfach wieder eine neue Gattung zu gründen. Doch dürfte es zweckmässiger sein, sie bei den bekannten Genera einzurangieren und deren Definition anders zu begrenzen. Sie vereinigen einzelne Merkmale von Nctrostoma mit andern von Halipetasus und lassen ferner weitere von L. S. Schultze als zwischen beiden unterscheidend aufgeführte Kennzeichen als individuell oder mit dem Alter wechselnd erscheinen. Es sind daher die Schultze'schen Gattungen höchstens spezifisch verschieden [wenn nicht vielleicht uberhaupt identisch s. u.], es ist als Gattungsname der zuerst genannte Nctrostoma zu wählen.

Bei beiden sind die Mundarme wiederholt gabelteilig, bei Netrostoma sollen sie lateral stark comprimirt sein, bei Halipetaszs dorso-ventral; doch ist, wie mir aus der guten eigenen Beschreibung L. S. Sciut.tzE's hervorzugehen scheint, diese Abplattung nur dadurch hervorgerufen, "dass sich die Saugkrausen auf die Seiten des Armstammes und seiner Gabeläste beträchtlich ausdehnen" (1898, p. 459). Auch hier sind die Saugkrausen sehr ausgedehnt im Verhältniss zum gallertigen Armteil (Fig. 37-40), und es kommt dadurch eine Anordnung wie bei Halipctasus beschrieben "in compacten, blumenkohlartigen Büscheln" zu stande; die Arme selbst sind aber 
seitlich comprimirt, wie bei Netrostoma mit deutlicher abaxialer (dorsaler) Kante, die schon auf dem walzigen Oberarm beginnt. Von besonderen Anhängen fehlen beiden Gattungen die für Cephea angegebenen "Peitschenfilamente" vollkommen; dagegen hat $N$. in der Armscheibe an jeder Gabelstelle einen grossen spindelförmigen Anhang; zwischen den Armkrausen selbst finden sich keinerlei Anhänge. Umgekehrt fehlen bei $N$. in der Armscheibe solche spindelförmigen Anhänge; dagegen kommen daselbst und auf den Armen kleine, krausenlose Anhangsgebilde vor, und ein Arm trug auch einen grossen spindelförmigen Anhang. (Schultze hat nur e in H.-Exemplar von $6 \mathrm{~cm}$. und zwei $N$.-Exemplare von I I, und $4,5 \mathrm{~cm}$. Durchmesser zur Verfügung gehabt).

Was hier von Anhangsgebilden zu sehen ist, lässt die SchulrzE'schen Unterscheidungen sehr relativ erscheinen. Die Mitte der Armscheibe trägt sehr verschieden lange gallertige Anhangsgebilde mit Centralcanal (s. Fig. 45). Wo solche sehr reichlich und teilweise von besonderer Grösse (bis über $2 \mathrm{~cm}$. lang) ausgebildet waren, da handelte es sich um Weibchen, bei denen sich Embryonen in grosser Zahl hier angeheftet fanden, eine Brutphlege, die ja bei Rhizostomen sehr verbreitet ist; wo sie sehr spärlich vorhanden waren, waren es Männchen. Ausserdem finden sich hier auf den Armen, zwischen den Krausen selbst, zahlreiche kleinere gestielte Anhänge von kegel- und spindelförmiger Gestalt (Fig. 44), von den gestielten Saugnäpfen der Cotylorhiza sehr verschieden, die dem ganzen Arm ein charakteristisches Gepräge verleihen. Es sind dies wohl die von Schultze bei $H$. erwähnten Anhangsgebilde, die bei $\Lambda$. fehlen sollen, hier aber in sehr wechselnder Menge vorhanden sind.

Was das Canalsystem betrifft, so sollen bei $N$. scharf gesonderte Rhopalar- und 24 mit zahlreichen seitlichen Blindästen besetzte Nebenradiärcanäle vorhanden sein, während bei $H$. alle 32 Radiürcanäle gleichmässig verästelt sind. Nach L. S. SchultzE's eigenen Abbildungen aber (Taf. XXXIII, Fig. 7 und Taf. XXXIV, Fig. I 2) ist dieser Unterschied ein sehr geringfiugiger, und in der genaueren Textbeschreibung heisst es auch von $H$.; dass die rhopalaren Canäle sich auf ihrem Weg zum Schirmrand erst später verästeln als die interrhopalaren. Auch bei meinem Material sind die rhopalaren Canäle von der Nagenperipherie ab immer noch eine Strecke weiter zum Schirmrand zu verfolgen, wie die andern, die in dem allgemeinen Gefässnetz aufgehen; wie weit aber, das ist bei den einzelnen variabel und besonders bei Altersstadien verschieden, und hängt von der ebenfalls sich ändernden Blindsack- und Anastomosenbildung ab. Charakteristisch ist in allen Fällen, dass die Netzbildung nur ganz allmählich gegen die Peripherie zu enger wird, und nicht wie bei andern Gruppen ein weit- und ein engmaschiger Bezirk deutlich geschieden sind, ferner dass kein Ringcanal vorhanden ist.

Ein ferneres Unterscheidungsmerkmal soll in der Structur der Exumbrella liegen. Bei $H$. ist dieselbe nur mit kleinen zerstreuten Körnern besetzt, und Schirmkranz und Kuppel sinc̉ nicht gesondert; bei $N$. trägt die Exumbrella grosse abgeplattete WVarzen, und Schirmkranz und Kuppel sind deutlich gesondert. Hierin kann ich nach den mir vorliegenden, verschieden grossen Exemplaren nur einen Altersunterschied erblicken. Das grösste Exemplar von $20 \mathrm{~cm}$. zeigt sehr grosse Warzen auf einem nur schwach abgesetzten Mittelfeld, und schwächere Randwarzen, das zweite mit ungefähr I $8 \mathrm{~cm}$. Schirmdurchmesser besitzt eine sehr ausgesprochene Schirmkuppel, mit lappigen Warzen, die peripher kleiner werden und sich verlieren (Iig. 4I); das kleinste Tier mit etwa $7 \mathrm{~cm}$. Durchmesser zeigt nur eine Anzahl rauher Körner auf der SIBOGA-EXPEDITIE XI. 
Exumbrella, die nach der Mitte zu etwas grösser werden. Dazwischen finden sich Uebergänge, und je nachdem die centralen grossen Warzen sich ausbilden, destomehr hebt sich auch eine besondere Schirmkuppel ab, eben durch die Warzen gebildet. Auch was L. S. Schultze vom Canalsystem beschreibt, das bei $H$. "viel einfacher" ist, bestärkt die Annahme von Altersunterschieden, ebenso seine Darstellung der Subgenitalostien. Diese sind bei $H$. kreisrund, bei $\lambda$. schwach trichterförmig. Ich konnte bei meinen Exemplaren beobachten, dass sich die anfangs nur kreisrunde Oeffnung eines Subgenitalraums mit zunehmendem Wachstum der Armscheibengallerte immer mehr zu einem Trichter auszieht, der bei meinem grössten Exemplar fast $\mathrm{I}_{1}^{1} / 2 \mathrm{~cm}$. lang wird. Auch dass bei $2 V$. der Schirmrand ausgeschweift ist, und die 8 Velarlappen zwischen je zwei Rhopalarlappen weniger scharf eingeschnitten und durch einen Randsaum verbunden und eventuell mit den Rhopalarlappen verwachsen sind, spricht dafür, dass $N$. gegenüber $H$. die ausgewachsenere Form ist, und in der übrigen Beschreibung Schultze's auch in den angegebenen Massen, spricht nichts dagegen. Immerhin könnte, wenn auch die generischen Merkmale sich z. T. als Wachstumsunterschiede erwiesen haben, doch wegen der Armgestalt und anderer kleinerer Differenzen, die spezifische Trennung einstweilen beibehalten werden, um so mehr als die Siboga-Form hierin ebenfalls Unterschiede aufweist, ebenso wie die von A. Agassiz und MIAYer abgebildete Cophea dumokuroc. Alle + wären dann Spezies einer Gattung.

Dass dumokuroa in der That den hier geschilderten Formen sehr nahe steht, ergiebt sich mehr noch aus den Abbildungen MAYER's (I899, Taf. I I), als aus der sehr summarischen Beschreibung. Die Mundarme sind zwar nur einmal gabelig geteilt wie bei Cephea, geben aber zahlreiche Seitenästchen ab und bei der gedrungenen Gesammtform der Arme nähern sie sich der Ausprägung der Siboga-Art. Auch ist die wiederholte Gabelteilung kein solch prinzipielles Merkmal; denn es kommt selbst bei Cephea laut HaEckel und Agassiz vor, dass die Gabellappen distal wieder in zwei kleine Läppchen gespalten sind, so dass z.B. die Species C. conifor $\alpha$ "schon einen Uebergang zur folgenden Gattung (Polyrhiza) herstellt" ( I 879, p. 576). Die langen Peitschenfilamente, die für Ccphca als charakteristisch angegeben werden, fehlen hier bei dumokuroa vollkommen. Kleinere, spindelförmige Anhänge könnten übersehen worden sein, zumal wenn sie, wie bei der Gattung Netrostoma, mitunter nur in spärlicher Zahl vorhanden sind. Die Schirmkuppel ist sehr ausgeprägt und zeigt ,dicke konische Hervorragungen"; von den 32 Radiärgefässen sind nicht 8 , sondern nur 4 durch Caliber und geringere Verästelung besonders ausgezeichnet. Will man also nicht Cephea in einem ganz andern und viel weiteren Sinn als bisher fassen, die alle Gattungen vereinigen würde, oder will man nicht wieder eine neue Gattung machen, so muss man dumokuroa wie oben erwähnt unterbringen. Durch VANHöffeN wird neuerdings eine Cephca cocmulca von der ost-afrikanischen Küste beschrieben und die Agassiz und MAYer'sche dumokmroc als Cephea gerechnet (1902, p. 46). Man könnte sich darum auch die Frage vorlegen, ob man die hier vorliegende Form nicht bei Cephea einordnen solle; doch hiesse das den wichtigsten Merkmalen, Armverzweigung und Peitschenfilamenten, Zwang an zu thun. Bei der Armverzweigung wäre der Abstand aus oben angefuihrtem Grund (Spaltung bei C.conifcra) noch nicht einmal so gross; aber von Peitschenfilamenten ist hier, so wenig wie bei den Schultze'schen Formen eine Spur vorhanden. Die spindelförmigen, starren Anhänge, die hier auf den Armen und in besonderer Grösse im Centrum der Armscheibe sitzen, können 
nicht als "Filamente" gedeutet werden. Auch wenn diese bei Cephca im Centrum manchmal sehr stark werden (wie eine "Taubenfeder'), so werden doch bei allen Arten, auch bei der VAxHöfFEx'schen, richtige Filamente auf den Armen beschrieben, die auch eine sehr beträchtliche Länge erreichen. Auch existieren weitere Unterschiede von den Cephea-Arten incl. der. VANHöFfEx'schen. Alle haben Kranzfurche der Exumbrella, zahlreiche (nicht nur 3 ) Radiärcanäle zwischen den 8 Rhopalarcanälen und viel weniger ausgesprochene Randlappen. Da sich diesen Merkmalen noch der Mangel der Peitschenfrlamente sowie die ausgebildetere Arm verzweigung dazugesellt, so möchte ich die vorliegende Form nicht unter Cephea rechnen ebensowenig wie dumokuroa, sondern beide dem Scruultze'schen neuen Genus, in etwas modifiziertem Sinn einordnen. Was die übrigen Gattungen der Gruppe Rh. dichotoma betriff, so sind in Polyrhiza sämmtliche 3 bei HAECKel aufgenannten Arten nur nach sehr alten Beschreibungen und Abbildungen aufgefuilırt; $P$. vesiculosa sogar nur nach einer blossen Diagnose Ehrenberg's, $P$. homopneusis nach einer von Lessox für eine Nacktschnecke gegebenen Figur, und $P$. orithya nach einer "sehr unvollständigen..... und unrichtigen" Abbildung von Quoy und Gammard, und keine von den dreien ist jemals wieder gefunden worden. Mir scheint die ganze Gattung mit ihrer Exumbrellastructur sehr problematisch. Aehnliches gilt von der Gattung Stylortiza mit ihren beiden Arten; hier wird zudem noch ein sehr breiter Spalt als Eingang in den. Subgenitalhohlraum beschreiben, während sonst für alle dichotoma eine enge kreisrunde Oeffnung das typische und verwertbare Merkmal ist. Es könnte angeregt werden, den einen oder andern Gattungsnamen, um einen neuen zu sparen, nur in etwas verändertem Sinn, für Formen mit wiederholter Teilung der Mundarme beizubehalten; doch wird dies zu einer Nomenclaturfrage, die namentlich mit Rücksicht auf die frühere Verwirrung (vgl. HAEckeL's Bemerkungen zu Ccphea und Styloriziza (p. 573 und p. 6I3) schwer zu entscheiden ist. Als sichere Gattungen der dichotoma bleiben: Cephea mit einfacher Gabelteilung der Mundarme, höckeriger Exumbrella und sehr langen Peitschenfilamenten, Cotylorfiza mit einfacher Gabelteilung, glatter Exumbrella und gestielten Saugnäpfen zwischen den Armkrausen und endlich Netrostoma. Deren Diagnose wäre dann folgendermassen zu fassen.

Netrostoma L. S. Schultze isgs.

Sensu amplif. 1902.

Microstylus? Kishinouye r902.

Mundarme mit einer Hauptgabelung und secundären kleineren dichotomischen Aestchen ohne Peitschenfilamente. Zwischen den Armkrausen und in der Mitte der Armscheibe längere und kürzere spindelförmige Anhänge. Exumbrella mit Warzen resp. Höckern.

[Die übrigen Merkmale: Canalsystem, Subgenitalostien etc. sind der ganze Gruppe gemeinsam und werden noch unten Besprechung finden].

I. Netrostoma cocrulescens spec. nov. (Taf. V, Fig. 37-46. Taf. XI, Fig. 97 und 103. Taf. XII, Fig. I09).

Stat. 209. 9 Exemplare von 7 , von 10-12 und von 14 cni. Schirmdurchmesser:

Sanguisiapo. I Exemplar von I8 cm. Schirmdurchmesser.

Ambon. I Exemplar von $20 \mathrm{~cm}$. 
Die Hauptunterschiede gegenüber den andern Arten liegen abgesehen von der wechselnden Gestalt des Subgenitalraums besonders in der Configuration der Arme. Da deren Gestalt hier auch für die Ableitung der verschiedenen Armtypen der Rhizostomeen i. Allg. wichtig ist, so ist eine ausfuhrlichere Beschreibung angezeigt. Die Arme zeichnen sich aus durch ihre gedrungene massige Form, sowie durch eine starke Krummung, besonders im distalen Teil (Taf. V, Fig. $37 \mathrm{z}$ ). Dadurch ist letzterer mit seiner Krausenunterseite dem äussern Teil des Oberarms wieder ganz nahe gerückt. Der dicke Oberarm misst bei dem grössten Exemplar $21 / \mathrm{cm}$. im Durchmesser, wo er von der 8 kantigen Armscheibe abgeht. Die Kante setzt sich auch noch auf der Aussenseite des cylindrischen, etwas seitlich comprimirten Oberarms und von da auf die Unterarmfligel fort. Die Hauptgabelung ist in der natürlichen Stellung kaum zu erkennen wegen des dichten ventralen Krausenbesatzes, der auch ganz um die Seiten der Arme herübergreift; man muss, um sie deutlich zu machen, die beiden Flügel von der abaxialen Seite gewaltsam auseinander spreizen, wie es Fig. 38 zeigt. Der Krausenbesatz des Oberarms zeigt ron der Subumbrella aus gesehen (Fig. 46) eine deutliche Fiederung; auf der Armscheibe sitzen diese Fiederästchen direkt auf, weiter distalwärts sind sie jedoch gestielt (Fig. 37 und Fig. 46) ähnlich wie für dumokuroa abgebildet (1899, pl. 12), und erlangen dadurch eine grössere Selbständigkeit, ohne jedoch der Zweigabelung des gesammten Oberarms gegenüber ins Gewicht zu fallen. Auch Schultze sagt für Netrostoma (1898, p. 458) "einzelne Krausengruppen des Oberarms können eine Selbständigkeit erreichen, die bei abgebrochenenem Gabelende des Arms eine fiedrige Verzweigung vortäuschen kann". Auffällig ist, wie leicht dieses Gabelende (ungefähr an der mit X bezeichneten Stelle Fig. 37 und Fig. 39) abbricht, was jedenfalls mit der plötzlichen Aenderung der Gallertstärke an dieser Stelle, sowie mit der Sprödigkeit infolge der starken Krimmung in Zusammenhang steht. Die stark gekrümmte Form der Arme im Verein mit der Zweiflügligkeit ist deshalb wichtig, weil sie einen Uebergang zu wirklich tripteren ArmFormen zu construiren erlaubt; man braucht sich nur die gleichmässige Krümmung an einer Stelle (Fig. $37 \mathrm{y}$ ) scharf geknickt zu denken und es ist eine Armform ähnlich wie bei Lychnorhiza, Versura etc. erreicht. Andrerseits ist die cylindrische Form des Oberarms und seiner Seitenästchen von Bedeutung, weil sie einen Uebergang zu Verzweigung wie bei Cassiopcja vermitteln.

Schwerer noch als die Hauptgabelung sind die Endgabelästchen zur Anschauung zu bringen; von der Unterseite erscheint nur ein dichter einheitlicher, blumenkohlartiger Krausenbesatz (Fig. 40, Fig. IO9); man muss das (am besten abgeschnittene) Gabelende auf einer Unterlage ausbreiten und von der abaxialen Seite aus betrachten; dann erscheinen (Fig. 39) die klcinen Endästchen mit ihrer Zweiteilung (Z! u.Z!!) recht deutlich. An Grösse sind sie jedoch so verschwindend (noch kleiner wie die Fiederäste des Oberarms) gegen die Hauptgabeläste, dass es mir unrichtig erscheint, einfach von wiederholter Zweigabelung zu sprechen, sondern dass man die Diagnose wie oben angegeben (e ine Hauptgabelung mit secundären, i. Allg. Dichotomie zeigenden, Nebenästchen) zu fassen hat.

Die viel umstrittenen Subgenit alräume erweisen sich, wie mir thatsächlich ausgeführte Halb-Schnitte, nicht blosse Sonden-Untersuchung zeigt, bei verschieden alten Tieren verschieden. Bei jungen Tieren sind es vier ganz getrennte Höhlen, in die man im Interradius durch je ein 
kleines, sich schnell erweiterndes Eingangsloch (Fig. 37 ost. gen.) hineingelangt, im Perradius stehen starke trennende Gallertpfeiler. Bei älteren Exemplaren reichen diese Pfeiler nicht mehr durch den ganzen Perradius hindurch, sondern bilden Coulissen oder Strebepfeiler eines gemeinsamen Hohlraumes, indem die interradialen Räume sich ausgedehnt haben. Wie weit, das ist eventuell sogar in den 4 verschiedenen Radien eines und desselben Exemplars verschieden. Auch beim ältesten von mir untersuchten Tier mit Embryonen waren noch perradiale Gallertstiucke als Stützen des "Subgenitalsaals" erhalten; zur Bildung eines völlig freien Hohlraums wie bei Crambessa scheint es hier nie zu kommen.

Das Canalsystem und seine Gefässnetzbildung ist bereits oben erörtert worden. Zur Erläuterung seien noch von einem mittelgrossen Exemplar die genau wiedergegebene Verzweigung der Haupt- und Nebencanäle (Taf. V, Fig. 43a) und von einem älteren Tier der Abgang der Gefässe von der (interradial ausgebuchteten) Magenperipherie (Fig. 43) abgebildet. V gl. auch Taf. XII, Fïg. IOO.

Die Muskulatur zeigt die von den Autoren beschriebenen Radialzüge in einzelnen Radien besonders ausgebildet; an den grösseren Exemplaren stärker und in förmlichen radiären Bündeln wie bei Cotylorhiza. Die Circulärmuskulatur ist auffallend schwach; nur in einiger Entfernung vom Schirmrand etwas deutlicher.

Die Anhangsgebilde zwischen den Saugkrausen der Mundarme zeichnen sich durch den plötzlich sehr erweiterten Centralkanal aus (Fig. 44). Die an der Armscheibe sind von zweierlei Art (Fig. 45), kleinere dünne Röhrchen von grosser Beweglichkeit, mit schwach entwickelter Zwischensubstanz zwischen dem nesselbewehrten Ectoderm (nw) und dem Centralcanál und grössere, starrere Gebilde mit starker Gallerte(pf). Ausserdem zeigt die Abbildung noch die gewöhnlichen Saugmündchen (kr) mit ihrer Verästelung und dazwischen zahlreiche Embryonen, die sich gelegentlich auch auf den peripheren Anhangsgebilden finden.

Die Randlappen sind weniger ausgeprägt, wie bei anderen Rhizostomeen-Gruppen, aber immerhin mehr wie bei Cephca selbst, wo meistens zwischen den Rhopalien nur ein ausgeschweifter Saum mit Andeutungen von Kerben vorhanden ist. Hier sind $6-8$ Lappen pro Octant deutlich zu zählen, die auch bei den grössten Exemplaren nicht verwischt sind.

Die Rhopalien zeichnen sich, wie bei allen dichotomen Rhizostomeen durch Einfachheit aus. Der Kolben ist gross, gestreckt (Taf. XI, Fig. 97), zeigt eine kleine Aussackung als Bulbus nach der Einengung, aber keine Abschnürung des Concrement-tragenden Endes. Dorsales und ventrales Nervenepithel (ect!), ersteres mehr distal, letzteres ungefähr am Bulbus sind deutlich zu erkennen, sonst aber keine Differenzierung. Es fehlt jede Pigmentandeutung, und es ist auch keine äussere Sinnesgrube entwickelt (s. Fig. IO3). Hierin stimmen also die Kolben mit der Beschreibung Hesse's von der verwandten Cotylorhica, und zeigen sich die dichotoma als die einfachsten Rhizostomeen.

Die Färbung scheint auf bestimmte Teile beschränkt zu sein, die sich aus der bläulichmilchigen Gallerte herausheben. Nach letzterer ist der Name gegeben. Ein brennrotes, fein zickzackförmiges Band verläuft auf der Aussenseite der Armscheibe, je nach deren starker Wölbung etwas auf und niedersteigend im Per- und Interradius (Fig. 37). Ansammlungen von kleinen ovalen braunen Flecken stehen zur Seite jedes Genitalostiums. Die entodermalen 
Teile schimmern (ihnlich wie bei Aurclia) violett durch die Gallerte, manchmal sehr dunkel und deutlich, manchmal in Folge des Formols stark verblasst.

Die Fortpflanzung geschieht in Verbindung mit der bei Rhizostomen so vielfach bekannten Butpllege. Einige $q$ Exemplare sind von Embryonen in der Armscheibe und von vorgeschritteneren auch in der Subumbrella (Fig. 109) dicht erfullt.

Die Unterschiede von den bekannten Arten sind in der obigen Beschreibung erörtert. Wir hätten sonach $N$. typhlodendrizm L. S. Schultze, $N$. scaber L. S. Schultze von Amboina, $\Lambda$. dumokuroa A. Ag. und Mayer von den Fiji-Inseln und $N$. cocrulescens Maas von Sanguisiapo und Stat. 209; event. auch nur 4 Localvarietäten einer Art (dann typhlodendrium) zu unterscheiden, zu denen vielleicht auch die japanische Microstylus gehört.

b) Rhizostomata pinnata.

Cassiopeja Péron \& Lesueur isog.

Sensu emendato mihi.

(Taf. IV, Fig. 24-36. Taf. XI, Fig. 98 und 102. Taf. XII, Fig. I 12).

Mundarme mehrfach und unregelmässig verzweigt, mit ventralen, verhältnissmässig schmalen Saugkrausen und grösseren oder kleineren Kolbenblasen $z$ wischen denselben. Sinneskolben an Zahl sehr variabel, um 16 herum. Radiärcanäle etwa 32. Muskulatur der Subumbrella in Fiederarcaden angeordnet. Subgenitalostien enge, kreisrunde Löcher; 4 Subgenitalhöhlen durch breite Gallertpfeiler vollkommen getrennt.

Im Gegensatz zu anderen Rhizostomiden, wo oft die gleiche Gattung von verschiedenen Autoren verschieden umschrieben wird, sind Angehörige des Genus Cassiopeja stets als solche zu erkennen. Der charakteristische Habitus der baumförmig verästelten Mundarme (Fig. 26 und 27), die erhöhte, zwar variable, aber sich um I6 herum bewegende Zahl der Rhopalien und vor allem die merkwürdige Fiedermuskulatur in der Subumbrella (Fig. 30), die ich uibrigens auch bei einer fossilen Form aus Solenhofen, in genau gleicher Ausprägung wiederfand (1902), lassen die Zugehörigkeit zur Gruppe unzweifelhaft. Zu den obigen Modificationen der bisherigen Diagnose ist folgendes zu bemerken. Es werden bisher die Mundarme der Cassiopeja einfach als gefiedert bezeichnet und den dichotomisch gegabelten gegenüber gestellt. Jedoch kommt hier zwischen der Fiederung auch Gabelteilung vor. Der Unterarm zeigt zwar nie die charakteristischen zwei Haupt-Flügel wie bei Cotylorhisa und Cephea (s. o. p. 36), sondern verlängert sich selbst noch weiter gegen den Endast; am Ende selbst aber und in den seitlichen Fiederästen ist wiederholt Gabelteilung zu beobachten. Auch L. S. Scrultze erwähnt bei seiner C. cyclobalia ( 1898 , p. 460) "ein eigenartiges Gemisch von Fiederspaltigkeit und Dichotomie", das auch auf der Abbildung der Brandschen C. Mcrtensi (I 838 , Taf. XXI) zu sehen, wenn auch nicht so ausgesprochen sei. [Umgekehrt zeigen Formen mit typischer Zweigabelung der Arme wie Cotylorhiza an den Gabelästen öfters ausgesprochene Fiederung. Von der gegenseitigen Ableitung der Armform und der Krausenverhältnisse der Rhizostomen i. Allg. wird 
noch in einem besonderen Abschnitte zu reden sein]; der Unterschicd zwischen Zweigabelung (Rhizostomata dichotoma) und Fiederung (Rh. pinnata) kann zwar i. G. und für die erste Teilung der Unterarme aufrecht erhalten werden, doch wird die Fiederung hier undeutlich und darum (s. Fig. 27 und 28 etc.) in der Diagnose besser als unregelmässig und baumförmig bezeichnet.

Eine zweite Eigenschaft verleiht den Mundarmen der Cassiopeja ebenfalls einen charakteristischen Habitus: die geringe Ausdehnung der Krausen im Verhältniss zu den Armen resp. den Aesten. Damit meine ich nicht, dass die Krausen nur auf die ventrale Seite beschränkt bleiben; das ist ja auch bei Cephea, Halipetasus und andern dichotoma der Fall, wo sie trotzdem eine grosse büschelige Ausdehnung erlangen. Hier aber liegen sie fast nur in einer ventralen Mittelrinne in jedem Ast und Aestchen, ohne auf die Seiten des meist drehrunden Astes ubberzugreifen. Von der Subumbrellarseite aus kann nun, namentlich wenn die Gesammtform abgeplattet ist und bei reicher Verästelung, der Krausenbesatz scheinbar ausgedehnt und zusammenhängend sein (Fig. 29); von oben und seitlich gesehen erscheinen fast nur Armverästelungen (vgl. Fig. 27 und 28 mit 29).

Wie sehr die Zahl der Randkörper und der Marginalteile überhaupt variirt, die für die Gattung charakteristisch sein sollte, ist von MAYER und besonders von BiGELow ( I 900), und schon von L. S. Schultze (I898) genauer erörtert worden. Letzterer hat sich darum auch für Einbeziehung des Genus.Polyclonia L. Ag., die nur 12 Randkörper haben soll, ausgesprochen. Ich möchte das auf Grund vielfacher Zählungen bestätigen und auch eventuell die Gattung Torenma, die 8 Randkörper haben soll, bisher aber nur bei HAECKEL existiert, mit 3 Arten als Jugendstadien von Cassiopeja-Arten einrangieren. Die Trennung in vier einzelne Subgenitalräume möchte ich zwar nicht als ein für die ganze Systematik der Rhizostomiden entscheidendes Merkmal, wohl aber als für die engere Gruppe charakterisch hervorheben, noch mehr aber die enge, kreisrunde Oeffnung der Subgenitalostien und besonders die Fiedermuskulatur der Subumbrella.

Es sind im Ganzen von Cassiopeja selbst ro Arten beschrieben, ungerechnet die eine Polyclonia und die 3 Torenma. Sämmtliche weisen eine Randzeichnung der Exumbrella auf mit Ausnahme der $C$. depressa Hckl. Da es sich bei letzterer Form um eine Beschreibung nach von andern conserviertem Material handelt, die Zeichnung auch verwaschen sein kann, so ist es mehr als wahrscheinlich, dass die eigentiimlichen Exumbrellarflecke ebenfalls als Charakteristikum der gesammten Gattung dienen können.

Die Ausprägung der Zeichnung selbst soll bei den einzelnen Arten verschieden sein. Danach, sowie nach Zahl der Randlappen, nach Verhältniss von Armlänge und Schirmradius, nach Vorhandensein oder Fehlen grösserer Kolbenblasen zwischen den kleinen, werder die verschiedenen Arten abgegrenzt, alles, wie man sieht oder sehen wird, etwas problematische Merkmale. Die beschriebenen Species sind:

C. Andromeda Eschsch. 3 Lappen zwischen je 2 Rhopalien. Arme fast gleich Radius. Einzelne grosse Kolben.

C. ornata Hckl. 3 Lappen zwischen je 2 Rhopalien. Arme grösser als Radius. Nur kleine Kolbenbläschen. 
C. Mortensi Brdt. 6 Lappen zwischen je 2 Rhopalien. Arme etwa $i^{1} / 2$ Radius. Zahlreiche grosse Kolben.

C. depressa Hckl. 7 Lappen zwischen je 2 Rhopalien. Arme kürzer als Radius. Nur kleine Bläschen.

C. polypoides Keller. [Saugnapf in der Exumbrella]. 3 Lappen zwischen je 2 Rhopalien. Arme grösser als Radius. Grosse und kleine Kolben.

C. picta Vanhöffen. 5 Lappen zwischen je 2 Rhopalien. Arme kiurzer als Radius. Nur kleine Kolben.

C. cyclobalia L. S. Schultze, 3 Lappen zwischen je 2 Rhopalien. Arme ${ }^{3 / 5}$ Radius. Eine grosse Kolbenblase pro Arm.

C. ndrosia A. Ag. und Mayer. 2 Lappen zwischen je 2 Rhopalien. Arme $\mathrm{I}^{1} / 2$ Radius. Zahlreiche grosse Kolbenblasen.

C. andromcda var. zansibarica Chun. 4-6 Lappen zwischen je 2 Rhopalien. Arme ungefähr gleich Radius. Grosse und kleine Kolbenblasen.

Alle diese Formen sind indopacifisch, resp. aus dem roten Meer, dazu kommen.

C. Xamachana Bigelow. [Mit concaver Exumbrella]. 3 Lappen zwischen je 2 Rhopalien. Arme mehr als I $1 \%$ Radius. Einzelne grosse Kolbenblasen.

Polyclonia (Cassiopia) frondosa L. Ag. 7-9 Lappen zwischen 2 Rhopalien. Arme I1/2 Radius. Nur kleine Kolbenblasen.

Im Material der Siboga-Expedition finden sich an 9 Fundstellen zahlreichen Cassiopeien von sehr verschiedenem Alter, die kleinsten von weniger als I $\mathrm{cm}$. Durchmesser mit noch offenem Mund die grössten von ïber $20 \mathrm{~cm}$. Durchmesser mit teilweise entleerten Gonaden. Sie gehören jedenfalls zwei verschiedenen Formenkreisen an, lassen sich aber in keine der bestehenden Arten nach den bisherigen Merkmalen einreihen. Anstatt jedoch daraus 2 weitere neue Arten zu machen, erscheint es mir ratsamer, erst die vorhandenen Merkmale und Arten auf ihre Berechtigung zu prifen, die zwei neuen einstweilen nur als Varietäten zu beschreiben und dann eventuell eine Aenderung und Vereinfachung der Artgruppirung vorzuschlagen. Die beiden Siboga-Arten haben entsprechend obiger Tabelle folgende Merkmale:

C. Andromeda var. malayensis. I, 3, 5 oder 7 und mehr Lappen zwischen je 2 Rhopalien. Arme kleiner als Radius. Einzelne grosse Kolben (nicht auf jedem Arm).

C. omata var. digitata. [Radialstreifen auf der Subumbrella]. 3 oder unregelmässig viel Lappen zwischen je 2 Rhopalien. Arme etwa $1^{1 / 2}$ grösser als Radius. Nur ganz kleine Kolbenblasen.

Das vorangestellte Merkmal, die Zahl der Randlappen, ist nach Untersuchungen, die ich an zahlreichen Exemplaren angestellt habe, nicht nur individuell, sondern besonders in verschiedenen Altersstadien sehr variabel. Ganz abgesehen von der wechselnden Zahl der 
Rhopalien und Antimeren, die die Gesammtzählung der Lappen illusorisch macht, sodass Angaben wie 80, oder II 2 Randlappen ungenügend sind, ist auch die Zahl der Lappen im Antimer, also zwischen je zwei Rhopalien, die neuere Autoren dazu angeben, sehr wechselnd. Schon Chun bemerkt, dass sich der Schirmrand bei älteren Exemplaren derart ausflacht, dass es kaum möglich ist, die Zahl der Velarlappen zu bestimmen (IS96, p. I \&). Auch Bigelow erwähnt die wechselnde Zahl und die Interpolation der Randlappen (1900, p. I97); doch scheint er dies nur als anormal wie die Rhopalienzahl anzusehen und nicht als einen fortschreitenden Altersunterschied, sondern giebt in der Artdiagnose eine bestimmte Zahl an. L. S. Scrultrze vollends sieht in der Gliederung des Schirmrands und in der Zeichnung das wichtigste Artmerkmal ( 1897, p. I63). Es ist daher wohl nicht überflüsig, die von mir constatierten Altersunterschiede abzubilden (Fig. $3 \mathrm{I}-34$ von C. andromeda und Fig. 35 u. 36 von ornata).

An ganz jugendlichen Exemplaren von andromeda var. malaycnsis is nur e in Randlappen zwischen je zwei Sinneskörpern zu erkennen (Fig. 3I). Am deutlichsten markiert sich der Einschnitt eine Strecke vom Schirmrand entfernt durch tiefe Kerben (10), während ganz peripher ein Randsaum die Lappen verbindet, der nur seichte und unvollkommene, den Lappen nicht immer ganz entsprechende Einschnitte zeigt. Dagegen stimmt die Zahl der peripheren Taschen des Gefässsystems mit den Randlappen überein; nur zeigen sich hier die ersten Spuren der Weiterbildung und des Einschubs, nach ehe neue Lappen selbst gebildet sind. Schon am ersten abgebildeten Stadium sieht man, dass die eine Tasche einen, die andere zwei Blindsäcke aufweist (Fig. 3I) und dadurch die Bildung neuer Lappen vorbereitet wird. Im späteren und länger dauernden Stadium sind drei vollständige Taschen zwischen je zwei Rhopalartaschenhälften zu erkennen (Fig. 32); auf der einen Seite der Figur entsprechen den 3 Taschen auch 3 Lappeneinschnitte, auf der andern ist der dritte Einschnitt entsprechend der Tasche noch nicht ganz ausgeprägt. Weiterhin (Fig. 33) erkennt man 3 sehr deutlich eingeschnittene Lappen, die zugehörigen 3 Taschen beginnen sich aber auf mannigfaltige Art zu teilen. An einem etwas reguläreren späten Stadium endlich (Fig. 34) sieht man 5 gleich grosse Taschen zwischen den Rhopalartaschenhälften; die innere Einkerbung des Schirmrands erinnert noch an die frihere Dreiteilung, weist aber bereits zwei weitere Einkerbungen auf. Aehnlich gestaltet sich auch die Bildung bei der zweiten von mir untersuchten Form (omata var. digitata); nur ist hier die Taschenbildung viel unregelmässiger, man findet oft zwischen zwei Rhopalien ein nahezu gleichmässig ausgebogenes peripheres Canalsystem mit kleinen unregelmässigen Blindsäckchen und einem continuirlichen Randsaum (Fig. 35). Weiter centralwärts zeigen sich noch die Kerben der Dreiteilung. In andern Fällen sieht man auch noch 3 einzelne Lappentaschen mit kleinen Ausbuchtungen (Fig. 36). Dann folgen auch hier weitere Einkerbungen des inneren Schirmrandes nach. Es bedarf also wohl keiner weiteren Erörterung, dass die Einteilung cles Schirmrands nicht als Artmerkmal dienen kann.

Ebensowenig kann die bis jetzt dazu benutzte Exumbrellarzeichung dazu herhalten. Die Zahl der Radialflecken, die den Rand der Exumbrella verzieren, entspricht der der Randlappen und ist mithin wechselnd. Ebenso die Gestalt der Flecke, die manchmal melur lanzettförmig, manchmal mehr eiförmig, manchmal mehr bandförmig sein soll. Schon daraus, dass öfters benachbarte Radialflecke zu einem einzigen verschmelzen, geht hervor, dass ihre Form nicht 
fest bestimmt ist; noch mehr daraus, dass sie öfters (und nach dem Centrum zu stets) verwaschen sind, und die Schärfe der Zeichnung zugleich mit der Gesammtfärbung der Tentakelblasen etc. wechselt. KeLleR hat bei seiner polypoides 5 solcher Varietäten unterschieden ( $188_{3}$, p. 634) und auch Chux, Bigelow und $A$. heben die Farbenvariabilität hervor. Die von mir untersuchte Form malaycnsis zeigt an den Formolexemplaren ausser an den Kolbenblasen, die noch eine violette Sprenkelung bewahrt haben, überhaupt keine Färbung mehr; doch kann dies wie bei depressa oben erwähnt, durch den Erhaltungszustand bedingt sein. Ich habe an den Riffen der Küste von Cypern Cassiopcia andromeda selbst gesammelt und die verschieden gestalteten, meist rhombischen Radialflecke notiert. Die conservierten Exemplare zeigen aber davon, wie die hier vorliegenden, nur Schatten. Eine andere Frage wäre, ob das Vorkommen einer Zeichnung auf der Subumbrella, das nur für einzelne Species (ornata, picta und polypoides) erwähnt wird, und das auch bei der zweiten Siboga-form var. digitaía zu finden ist, als solches einen unterscheidenden Charakter hat. Jedenfalls ist es nur im Verein mit andern Merkmalen zu beniitzen.

Von solchen bleiben noch das Vorhandensein oder Fehlen von grossen Kolbenblasen. Dies scheint thatsächlich einen Unterschied zu begründen. Die grossen Kolben sind so auffällige Bildungen, mitunter fast die Länge eines Arms erreichend, dass sie auch den alten Beschreibern zur Kennzeichnung gedient haben, und sie sind, wenn auch an Zahl und Regelmässigkeit verschieden, doch immer in Mehrzahl vorhanden, so dass man sich ihr ausdriicklich hervorgehobenes Fehlen nicht durch Abgerissen sein erklären kann. Nicht zu verwechseln ist damit die Rosette von kleinen Blasen, die sich beim Weibchen im Centrum der Armscheibe befindet, da wo beim Männchen die Mundnahtlinien und ihr Krausenbesatz in der bekannten Kreuzfigur zusammenstossen. Haecker war der Meinung, dass diese kleinen Kolben in jugendlichem Individuen stets vorhanden seien, bei alten Tieren aber nabgerieben" würden (1879, p. 571) und giebt auch entsprechende Abbildungen. Dass es sich hierbei um einen Sexualunterschied handelt, haben spätere Autoren gefunden. Die kleinen Kolben dienen, wie namentlich BIGELow beschreibt, zur Anheftung der Embryonen, so dass die Mutter eine Art Brutpflege ausibt.

Ein durchgreifender Unterschied ist ferner in den Verhältniss von Armlänge zum Schirmradius gegeben. Es erscheint dies zunächst als etwas sehr relatives; ein Vergleich der Diagnosen zeigt jedoch, dass durchweg entweder angegeben ist, dass der Arm merklich ( $\mathrm{r}^{1} / 2$ mal und mehr) länger als der Schirmradius ist, oder deutlich kïrzer und aus der Subumbrella nicht herausragt. Und damit gehen andere Unterschiede in der Armverzweigung und Ausbreitung parallel. Bei längerem Arm ist die Fiederung stets deutlich ausgesprochen; die Aeste sind im spitzen Winkei zur Subumbrella ausgebreitet, oft in verschiedenen Richtungen gewunden, im Querschnitt cylindrisch oder etwas seitlich comprimirt. Bei kürzerem Arm ist die Fiederung nicht rein, sondern mit Dichotomie, namentlich an den Endästchen, verbunden; die Aeste sind fast in einer Ebene ausgebreitet ${ }^{1}$ ) und von flacherer, dorso-ventral-comprimirter Form. Besser als lange Beschreibung mögen Fig. 26 den ersten, und Fig. 27 den andern Fall erläutern, oder die Figuren von andromeda und ornata der Autoren. 
Das Vorhandensein eines "Saugnapfes" oder wenigstens einer Delle in der Exumbrella kann bei zwei Arten immerhin noch als ein weiteres Merkmal gelten, da bei allen andern Species ausdrücklich eine mehr oder minder gewölbte Schirmform, manchmal zwar recht flach (s. Fig. 24) aber doch ohne besondere Grube, beschrieben und abgebildet wird. Die Concavität ist, wenn vorhanden, als eine besondere Ausbildung und Folge der allen Cassiopeien eigentümlichen Lebensweise aufzufassen. Dieselben sind keine planktonischen Tiere, sondern gehören dem Benthos an. Sie ruhen in der flachen Strandzone auf dem Boden, mit der Armscheibe nach oben gekehrt; haben aber, wie sich beim Aufstören zeigt, ihre Schwimmfähigkeit nicht eingebüsst. Da es sich also nicht um pelagische Medusen, sondern um richtige Ufertiere handelt, so ist es eher anzunehmen, dass sich verschiedene Localformen in verschiedenen geographischen Gebieten finden können. Es dürfte jedoch nicht allen beschriebenen der Wert von Arten zu kommen, sondern sich, namentlich, wo nur Färbung und Zeichnung in Betracht kommt (die Randlappenzahl fällt ja weg) nur um Varietäten handeln und sich die bisher beschriebenen nebst den neuen folgendermassen gruppieren:
A. Mit langen, rein fiedrigen, cylindrischen Armen.
B. Nit kurzen, unregelmässig verzweigten, flachen Armen.

1. Mit grossen Kolbenblasen.

\%. ohne Saugnapf der Exumbrella.

C. mertensi Brandt.

var. ndrosia A. Ag. und Mayer.

ק. mit Saugnapf, resp. Concavität.

C. polypoides Keller.

C. xamachana Bigelow.

1. Mit grossen Kolbenblasen.

C. andromeda Eschscholtz.

var. zanzibarica Chun.

var. cyclobalia L. S. Schultze.

var. malayensis NIaas.

2. Ohne grosse Kolbenblasen.

C. depressa Haeckel.

var. picta Vanhöffen.

2. Ohne grosse Kolbenblasen.

C. ornata Haeckel.

var. digitata Maas.

Hierher ev. Polyclonia (C.) frondosa.

Damit reducieren sich die besprochenen I 3 Arten auf 6 resp. 7 . Ob nicht noch weitere Reductionen zu machen sind, und ob nicht die ganze Gattung Torcuma mit ihren 3 HAEckeLschen Arten zu fallen hat, möge späterem Material vorbehalten bleiben, und hier noch einiges Detail ron den durch die Siboga gefundenen Cassiopcia-formen aufgefithrt werden.

\section{Cassiopeja andromeda nov. var. malayensis.}

(Taf. IV, Fig, 24-34 excl. 26 und 30. Taf. XI, Fig. 98 und 102. Taf. XII, Fig. I I 2 )

Schirmform i. G. sehr flach mit leichter ringförmiger Einsenkung in der Gegend der Magenperipherie (Fig. 24). Zwischen 2 Rhopalien I, 3, 5 und mehr Randlappen. Subumbrella ohne Zeichnung. Arme etwas kürzer als der Schirmradius, dorso-ventral comprimirt. Grosse Kolbenblasen vorhanden, meist aber nur spärlich, nicht auf jedem Arm; manchmal nur 2 oder 
3 am ganzen Tier. Saugkrausen von wechselnder Farbe (bräunlich-graugriin); grosse und kleine Kolbenblasen mit violetter Zeichnung (s. Fig. $29 \mathrm{ko}$ ).

Stat. 2. $0^{7}$ Exemplare von ïber $20 \mathrm{~cm}$. und $6 \mathrm{~cm}$. Durchmesser.

Stat. 64. 3 Exemplare von $\mathrm{I}, 2,3 \frac{1}{2} \mathrm{~cm}$. Durchmesser. Das kleinste mit noch offenem AIund.

Stat. 144. I Exemplar von $6 \mathrm{~cm}$. Durchmesser.

Stat. 205. I Exemplar von $21 / 2 \mathrm{~cm}$. Durchmesser. Schon mit Gonaden.

Stat. 258.9 Exemplare von $21 / 2-8 \mathrm{~cm}$. Durchmesser.

Stat. 26I. I Exemplar von etwa $10 \mathrm{~cm}$. Durchmesser.

Nusa laut. I Exemplar von $I, 8 \mathrm{~cm}$. Durchmesser. Mund noch nicht ganz vernäht.

Saleyer. 2 Exemplare von etwa 7 und $12 \mathrm{~cm}$. Durchmesser.

Die Varietität steht der L. S. Schultze'schen cyclobalia am nächsten, die Unterschiede von dieser und der typischen andromeda gehen aus obiger Diagnose hervor. Sie liegen besonders in der Form der Arme, die bei cyclobalia seitlich, hier dorso-ventral comprimirt sind, und in der Zeichnung. Eine besondere Schirmgrube ist hier nicht vorhanden und wird nur dadurch scheinbar hervorgerufen, dass der Schirm etwa in der Mitte des Radius der Exumbrella, wo ihn keine Armscheibe stiitzt, leicht einsinkt; manche Exemplare zeigen eine völlig wagrechte, wie mit Linie abgeschnittene Exumbrella. Es rührt dies sicher von der Lage im flachen Sandstrand, wie ich bei Exemplaren von Cypern (C. andromeda) selbst im Leben beobachten konnte.

Ueber den inneren Bau und besonders das Verhältnis der Gonaden zum Canalsystem geben Fig. 24 und 25, Schnitte durch Perradius und Interradius Aufschluss. In letzterem ist die enge Mündung des Subgenitalraums getroffen, der in jedem Interradius einen besonderen, wenig geräumigen Hohlraum darstellt. Vom benachbarten ist er, wie der Schnitt durch den Perradius zeigt, vollständig abgetrennt; hier erscheint die Gallerte continuirlich und sogar zwischen Mitte und Rand durch besondere Träger (gal per) verstärkt. Dieses Verhältnis ändert sich auch nicht bei alten und geschlechtsreifen Tieren. Die Decke eines Subgenitalraums wird in der bekannten Weise von der Gastrogenitalmembran gebildet; die Gonadenfalten selbst springen in den centralen Magenraum vor. Von oben gesehen stellen die Gonaden 4 getrennte Hufeisen dar pro Interradius, mit der Convexität nach innen, die sich aber bei reifen Tieren sehr ausdehnen und fast berihhren, so dass ihr Schnitt auf dem Perradius von dem im Interradius wenig verschieden ist.

Sehr auffällig sind durch Grösse und Stellung die Kolbenblasen; sie liegen hier nicht an der Gabelung der Arme, sondern ganz peripher so dass sie iiber den Schirmrand hinausragen. Nanchmal sind es mehrere und bei alten Exemplare viele, manchmal auch nur eine besonders grosse; auch sind die Arme sehr ungleich damit bedacht; manchen Armen fehlen sie ganz, und schon bei jungen Tieren sind diese Unregelmässigkeiten sehr ausgesprochen (Fig. I I 2). Dass der Subumbrellarmuskel auch einen proximalen Teil aus parallelen Ringziigen besitzt, hat L. S. Schultze mit Recht hervorgehoben. Auch bei einer fossilen Form habe ich diesen proximalen Teil, der den Magen umschnirt, beschrieben und abgebildet (I go2, Taf. XXIII). Der periphere Kranzmuskel, in den die Fiederarkaden auslaufen (s. Fig. $30 \mathrm{~m}$ cir) ist ebenfalls sehr deutlich.

Die Rhopalien sind innerhalb der Cassiopeiagruppe durchaus gleich gebildet, wie Vergleiche mit den Darstellungen von KELLER (I883) und BigELow (I900) zeigen, aber gegen- 
ïber den andern Rhizostomeen nicht ohne systematische Bedeutung. Bei den von mir untersuchten Exemplaren sind die Kolben im Verhältnis zu den Randeinschnitten auffallend klein (Taf. XI, Fig. 98) (vielleicht sind sie wie KELLER meint in Folge der sesshafteren Lebensweise reduciert). Sie sind gleichmässig dick; eine kleine Anschwellung ist nicht auf einen ventralen Bulbus, sondern nur auf Ectodermverstärkung zu rückzufihren (Fig. I02). Das Entoderm geht continuirlich bis zum Endteil mit den Otolithen. Fast ganz distal ist ein schwacher Pigmentfleck zu erkennen, der aber nur eine oberflächliche Färbung, keine Einsenkung in die Tiefe bedeutet. Eine äussere Sinnesgrube ist hier, wenn auch nur schwach entwickelt vorhanden: sie ist im Aufsichtsbild etwa sichelförmig, zeigt keine Radiärfalten; die sie zusammensetzenden Zellen sind nur nervöser und stiitzender, nicht drüsiger Natur, und nicht von besonderer Höhe.

Die Entwicklung geht wie zahlreiche anhängende Embryonen zeigen, am mütterlichen Körper bis zum Stadium einer stark differenzierten Planula.

\section{Cassiopeja ormata var. nov. digitata. (Taf. IV, Fig. 26 und 30).}

Schirm sehr flach, ohne Furche oder Kuppel. Randlappenzahl von 3 ab pro Antimer ganz unregelmässig. Exumbrella mit verwaschenen Radialflecken. Subumbrella mit i 6 ausgedehnten violetten Radialstreifen; Saugkrausen braun-violett; i. G. zu den Armen spärlich entwickelt. Armverzweigung fingerförmig; grosse Kolbenblasen gänzlich fehlend.

$\begin{array}{lll}\text { Stat. 50. } & \text { I Ex. Etwas über } 4 \mathrm{~cm} \text {. Durchmesser. } \\ \text { Stat. 26I. } & \text { I Ex. Etwas über Io } \mathrm{cm} \text {. Durchmesser. } \\ \text { Stat. Saleyer. I Ex. Etwas uber } 9 \mathrm{~cm} \text {. Durchmesser. }\end{array}$

Da Exemplare an manchen Stationen mit der andern Art zusammen vorkommen, so könnte die Vermutung auftauchen, ob es sich nicht nur Alters- oder Conservierungs oder Sexualunterschiede handelt. Alle drei Möglichkeiten sind auszuschliessen; die erste, weil von andromeda var. malayensis alle Altersstadien unter dem Material sich finden, ohne dass eine der ornata var. digitata gliche; die zweite, weil die beiden verschiedenen Arten auf gleiche Weise fixiert und aufbewahrt waren, und die dritte, weil eine Prüfung der Geschlechtsverhältnisse ergab, dass z.B. die bei den verschiedenen von Stat. Ellat Rif beides Männchen waren und andrerseits, dass bei malayensis sich Männchen und Weibchen finden, die aber keine andern, als die friher erwahnten Unterschiede im centralen Kolbenbesatz fü die Brutpflege aufweisen. Zudem spricht alles, was oben iiber die Species erörtert wurden, gegen eine solche Auslegung.

Von der typischen ornata, wie sie HAECKEL beschreibt, ist die V'arietät, abgesehen von der Färbung, vor allem durch die eigentümliche Armverzweigung unterschieden. Es ist der Hauptast, von dem die Fiedern ausgehen nicht so sehr als solcher ausgesprochen, sondern es erfolgt gleich eine Verästelung in 5 und mehr fast gleichwertige Aeste; der eine ist öfters kürzer und geht mehr proximalwärts $a b$, wie ein Daumen, die andern stellen die ubrigen Finger dar; daher die gewählte Name. Die Aeste bleiben dann auffallend lange ungeteilt und zeigen erst ganz am Ende wieder eine Polytomie mit spärlichem Krausenbesatz (Fig. 26). Trielleicht dass man späterhin eine wirkliche Art aus dieser Varietät machen kann. Auch die Radialstreifen der Subumbrella zeichnen sie aus; was sonst von Zeichungen der Unterseite erwähnt wird, sind nur Fortsetzungen der Exumbrellarflecke. Es sind nur halb so viel Streifen als Fiederarcaden vorhanden; sie bezeichnen den Verlauf eines Rhopalarcanals; sonst zeigt aber das periphere Canalsystem keinerlei Färbung. 
Was die geographische T'erbreitung der Cassiopeien betrifft, so sind sie erstens durchweg Bewohner der wärmeren Meere, zweitens ist auffällig, dass fast sämmtliche Arten dem indopacifischen Gebiet angehören; nur xamachana ist atlantlich (und Polyclonia frondosa). Jedoch muss man sich vor weitgehenden Schlüssen hüten; denn gerade die beiden am entlegensten aufgefundenen Species xamachana von Jamaica und polypoides aus dem roten Meer sind untereinander am ähnlichsten und haben allein die saugnapfartige Vertiefung der Exumbrella. Auch war aus dem Mittelmeer, dessen Medusenfauna doch am besten bekannt ist, bisher keine Cassiopeja verzeichnet (Cassiopeja borbonica ist nur Synonym für die bekannte Cotylor/iza tubcralata). Die Auffindung der andromeda bei Cypern ist darum von Bedeutung. Sonst rechnet ja das mediterrane Gebiet durchaus zum atlantischen; im vorliegenden Fall aber ist es fraglich, ob es sich nicht eher um eine Ueberführung aus dem roten Meer handelt. Im festsitzenden Scyphostomastadium wäre eine solche Verschleppung leicht zu denken. Auch die andern Arten sind meist an mehreren Küisten gefunden. Es existiert also neben der Möglichkeit der localen Ausprägung die Möglichkeit der Verschleppung, so dass dieser Befund kaum bei andern Acraspeden Analogien haben wird.

c) Klh. triptera.

Sens. em. = triptera + trigona Vanhöffen 1888.

Wenn ich auch vorläufig die TANmöfFEv'sche Einteilung der Rhizostomeen nach der Armform angenommen habe, so kann ich ihm doch in einer Hinsicht, nämlich bei der Spaltung des Begriffs dreifluglig in die Gruppe der triptera und trigona, wogegen sich auch L. S. Schultze I 898 erklärt, nicht folgen. Bei den ersten „berühren sich" laut seiner Definition, „die ventrale Krausenreihe und die der dorsalen Flügel erst am distalen Ende des Arms", bei den letzteren sind die "Arme dreikantig pyramidal, dicht mit Saugkrausen bedeckt". Ich kann diesen Unterschied nur für einen sehr relativen halten, der in verschiedenen Altersstadien und sogar in verschiedenen Armstrecken beim selben Individuum verwischt sein kann. Zu Beginn der Entwicklung stehen die Krausen nur auf den Kanten, kommen also erst auf der Spitze der Pyramide zusammen (triptere Form), später aber greifen sie mehr und mehr auf die Fläche uiber, so dass besonders am distalen Ende, wo sich doch die Kanten nähern und die Fläche verjüngt, die ganze Fläche mit Krausen besetzt erscheint (trigone Form). Der Name bezieht sich auf den Querschnitt; doch wechselt auch dieser entsprechend. Im oberen (proximalen) Teil sind die Fliggel tiefer eingeschnitten, nach unten zu wird der Einschnitt immer seichter und die Gallerte der abaxialen F'ügel mit der axialen zusammenhängender, so dass der Querschnitt schliesslich nur als eingebuchtetes Dreieck erscheint. Es geht also vom proximalen Ende zum distalen die dreiflüglige Form in die dreikantige über. Ferner kann ein allseitiger, auf die Flächen übergreifender Krausenbesatz dadurch hervorgerufen werden, dass die Kanten zahlreiche seitliche Aestchen bilden, deren Krausen dann sehr weit auf die seitlichen Flächen reichen (z.B. Lychnorhiza, Crossostoma). T'AnHöffen rechnet allerdings solche Formen zu den tripteren, aber die Aeste entspringen vielfach auf der Fläche selbst, sodass es kaum zu entscheiden ist, ob sie der axialen oder abaxialen Kante anzugeliedern sind. Auch hat die Gattung Mastigias, die VAnHöfres 
doch selbst als trigon, also zur andern Gruppe rechnet, einen genau gleichen Zerfall der Krausenkanten in einzelne Läppchen, wie die erwähnten "tripteren" Formen. Ausser Mastigias zählt noch Crambessa bei VAnHöFfEx zu den trigonen. Gerade bei dieser aber zeigt sich, dass der Krausenbesatz nicht so allseitig entwickelt ist, sondern seine Beziehung zu den drei Kanten stets noch deutlich offenbart, dass die Fligelform persistirt, und die Pyramidenform der Arme nur scheinbar durch Zusammenklappen der Flügel zustande kommt. Alle unten erwähnten Autoren über Crambessa zeigen das in Beschreibung und Abbildung; Lendenfeld sagt z.B. ausdruicklich für Cr. mosaica (I888, p. 236) ,der grössere Teil des Arms wird vom Unterarm gebildet, welcher eine regelmässige dreikantige Pyramide dargestellt, an deren Kanten die Krausen sitzen" und ferner (1.c. p. 237), „die freien Ränder dieser 3 Flügel werden von den Krausen gekrönt.... die Krausen der Dorsalflügel sowie jene des untern Teils des Ventralflügels sind so gross und so reich gefaltet, dass sie die Flügel auf denen sie sitzen, vollständig verhüllen. Der ganze Unterarm erscheint als eine solide wollige Nasse". Im normalen Zustand sind die beiden Flügel der 8 Arme zusammengeklappt wie auch bei Crambione (s. Fig. 47 und Fig. 73), so dass von der abaxialen Seite aus ein allseitiger Krausenbesatz vorgetäuscht wird, indem nur eine Rinne die Trennungslinie der Flügel angiebt und scheinbar eine abaxiale Aussenfläche einer Pyramide entsteht. Gerade diese Scheinfläche mit dichtem Krausenbesatz ist aber in normaler Haltung bei allen Armen nach aussen gekehrt, und so erklären sich die alten und modernen Habitusbilder und deren Deutung. Klappt man den Unterarm an der bezeichneten Rinne auseinander, so erscheinen Gallertflächen, die von Krausen noch freier sind, wie die beiden seitlichen. Eine wirklich trigone Armform entsteht eigentlich weder bei Mastigias noch Crambessa, sondern in einer anderen Gruppe, bei den lorifera, auf grössere Strecken des Arms; gerade hier aber lässt sich zeigen, wie am selben Arm trigone und triptere Eildung in einander übergeht (s. u.). So sprechen alle Gründe gegen eine solche künstliche Spaltung des Begriffs dreiflüglig in die Abteilungen tripter und trigon. Die dreiflügligen im erweiterten, resp. alten Sinn vor VANHöfFEN sind allerdings eine etwas verschiedenartige Gesellschaft und begreifen Formen, die bei HAECKEL in 3 Unterfamilien, und bei CLAUs in 2 Familien stehen; aber die VANHöFfEN'sche Einteilung entspricht hier weder der Gruppierung dieser Autoren, noch einer natürlichen Scheidung. Dies zeigt sich schon bei der Umräumung, die von VANHöFfEN mit Recht in mehreren der HAEckEL'schen Genera vorgenommen hat, und die danach teils zu tripteren teils zu trigonen Formen kamen. Auch betont er selbst die Aehnlichkeit und Verwandschaft der tripteren Lychnorhiza $(+$ Crambor-hiza) mit der trigonen Crambessa. Hierzu kann ich ein neues Genus Crambione fügen, das durchaus eine Mittelstellung zwischen beiden einnimmt, und ferner auf die Aehnlichkeit der "trigonen' Mastigias mit der tripteren Crossostoma hinweisen. Will man also Unterlassungen und Irrtümer vermeiden, so hat man bei der Bestimmung und Kennzeichnung hierher gehöriger Formen nicht blos die eine oder andere der VAxHöfFEx'schen zwei Gruppen vorzunehmen, sondern den Begriff dreiflüglig im alten, weiteren Sinne zu fassen. Nach Beschreibung der einzelnen Formen, die nach und nach Gelegenheit zur Discussion sämmtlicher Genera geben, empfiehlt es sich dann, die ganze Reihe mit den durchgreifend erkannten Merkmalen der Gattungen zusammen zu stellen und dann erst, je nach den Annäherungen, kleinere Untergruppen zu bilden. 
Crambione nov. gen.

Rhizostomee mit dreifligligen Mundarmen; Flügel mit secundären Seitenästen und dichtem, scheinbar allseitigen Krausenbesatz. Mit kleinen Saugkölbchen und starken Peitschenfilamenten, sowohl auf den Ober-, als auf den Unterarmen. Mit einheitlichem Genitalporticus, aber 4 engen, nierenförmig geschlitzten Ostien. Canalsystem mit 8 rhopalaren, bis zum Schirmrand gehenden, und 8 adradialen im Ringcanal endigenden Canälen; a usserhalb des Ringcanals ein engmaschiges Gefässnetz, innerhalb ein einfaches, weitmaschiges, blindes Netz, zwischen je 2 Radiärcanälen, das nicht mit dem Magen zusammenhängt. Muskulatur circulär, ohne radiale Unterbrechung. Rhopalien mit Augenfleck und äusserer Sinnesgrube.

Diese neue Gattung steht von den bekannten am nächsten bei Lychmor/iza und Crambessa; sie lässt sich aber mit keiner von beiden vereinigen, ohne die bisherigen Diagnosen völlig zu verändern, und auch bei andern näher und weiter stehenden Formen findet sich keine Beschreibung, die sich nur annähernd auf die vorliegende Art beziehen liesse. Mit Lychnor hiza, die ja auch HaEciels Crambor-hiza begreift (der event. Unterschied beider liegt nur im Subgenitalraum s. VANhöfFex, p. 29) hat sie in Gestalt und Anhängen der Arme grosse Aehnlichkeit; auch Muskulatur und A. stimmen überein. Jedoch hat Lychnor/hisa (und ganz übereinstimmend Cramborfiza) nach HAEckEL's wie VANHöffEx's Beschreibung ein so eigenartiges Canalnetz mit nur einfachen Blindcanälen ohne jedes intracirculäre Gefässnetz, und mit so auffallend breiten Anastomosen (Haeckel Taf. XXXIV, VAxhöffex Taf. III, Fig. 2 u. 3) dass an eine Vereinigung mit der hiervorliegenden Form nicht gedacht worden kann. Diese hat ein Canalnetz, das viel mehr mit der ebenfalls sehr eigentiumlichen Bildung ron Crambessa überein stimmt, wie sie Haeckel (1869) und Grenacher und Noll geschildert haben (1876, Fig. I3) aber doch auch wieder davon einige charakteristische Abweichungen zeigt (Fig. 48 und Fig. I 13). Es bedarf kaum einer Rechtfertigung, die systematische Bedeutung des Getässnetzes, die von HAECKEL und noch mehr von VANIÖFEEN vernachlässigt wird, hervorzuheben. Die Art der Verzweigung ist, wie ich auch bei Mastigias betont habe, so iberraschend constant, dass junge Exemplare bereits ganz den Typus der Erwachsenen aufweisen (s. u.). Auch hier zeigen junge Exemplare von nur $\mathrm{I}^{1} / 2 \mathrm{~cm}$. Schirmdurchmesser schon das typische Bild, und bei den riesigen mir vorliegenden Tieren von gegen 30 und $40 \mathrm{~cm}$. Durchmesser ist keinerlei Abweichung eingetreten, keine Vermehrung der Radiarcanäle, keine V'erbindung des blinden Maschennetzes mil denselben oder dem Magen, kaum eine Naschenvermehrung erfolgt, sondern nur eine Vergrösserung des Canaldurchmessers selbst, proportional dem Gesammtwachstum! Auch L. S. Schultze hat sich an verschiedenen Stellen sehr fuir eine bessere Verwertung des Canalsystems ausgesprochen; gerade bei seiner Crambessa palmipes (I898, p. 454), worauf noch zurück zukommen sein wird. Nähert sich also Crambione hierin der Crambessa (ohne jedoch ganz iibereinzustimmen), so ist sie völlig davon verschieden durch den Besitz der Armanhänge, nicht nur der kleinen Saugkölbchen, die zwischen den Krausen nur wenig hervorragen, sondern 
vor allem durch die langen Peitschenfilamente, die doch bei einer Crambessa gewiss niemand entgangen wären. In allen den zahlreichen und ausfuhrlichen Beschreibungen jedoch, die Crambessa-Arten gefunden haben (Huxley, Haeciel, Grenacher und Nold, Lendenfeld, Ciuux, Schultze, A. G. Mayer) findet sich von Filamenten kein Wort erwähnt, mitunter wird das Fehlen der Anhänge sogar ausdrücklich hervorgehoben. Weitere Unterschiede, (hier glatte Exumbrella, einheitliche Muskulatur) kommen dazu. Von den übrigen Gattungen der dreiflugligen Formen, wobei sowohl trigona wie triptera zu berücksichtigen sind, sind natürlich die Unterschiede in Armform und Anhängen, Canalsystem, Muskulatur, Porticus und Ostien noch viel grösser und aus der Diagnose (wie aus untenstehender Tabelle) leicht ersichtlich. Die Aufstellung einer neuen Gattung ist daher leider nicht zu umgehen.

I. Crambione mastigophora $12 . \mathrm{g}$. nov. spec. ${ }^{1}$ )

(Taf. VI, Fig. 47-53 incl. Taf. VIII, Fig. 7 I-74. Taf. XI, Fig. 100 u. 104. Taf. XII, Fig. I I3).

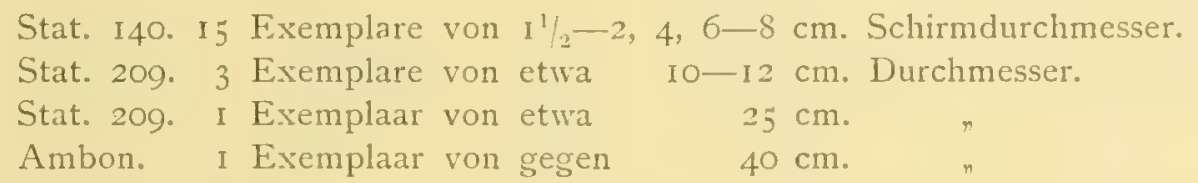

Der Schirm ist stark gewölbt, eine Strecke vom Schirmrand entfernt plötzlich und stark eingebogen; diese Zone bezeichnet den Uebergang der dicken Centralgallerte zur dünnen Randgallerte. Die Exumbrella ist glatt, gänzlich structur- und farblos; an jüngeren Exemplaren scheint das Gastrogenitalkreuz und das ganze Canalsystem durch die helle Gallerte hindurch; bei den grossen Exemplaren ist die Gallerte dicker und milchiger und lässt nur noch die Kreuzform der Gonaden schwach durchschimmern.

Die Armscheibe ist sehr breit, abgerundet, sodass die 8 Kanten (4 per-, 4 interradiale) fast zu einer Kreislinie sich vereinigen. Die Ostien und die Pfeiler wären nahezu gleich breit; doch sind die letzteren an der Basis etwas ausgerandet und vergrössert, die ersteren etwas eingeengt, sodass namentlich beim erwachsenen Exemplar die Pfeiler die Ostien merklich an Ausdehnung übertreffen. Durch wulstige lippenartige Gallerterhebung an der untern (distalen) und einen kleinen Gallertvorsprung an der oberen Seite sind die Genitalostien zu bogen- oder schlitzförmigen Oeffnungen verengert (Fig. 47 u. 50 ost gen.), die aber schon mit blossem Auge, noch besser mit der Sonde bis tief ins Innere verfolgt werden können. Ausser diesen 4 interradialen Spalten sind noch 4 perradiale, ganz ähnlich gestaltete, aber etwas höher sitzende (Fig. 47 und Fig. 50 fos per) von aussen auffällig. Diese haben jedoch keinerlei Beziehung zum Genitalsinus, sondern sind nur äusserliche, in die Gallerte eingegrabene Furchen, die nicht weiter in die Tiefe reichen. Solche Bildungen sind es jedenfalls, die in verschiedenen Fällen die Veranlassung gegeben haben, \& Atemhöhlen, resp. \& Genitalostien zu beschreiben; ein Irrtum, den HAEckel mehrfach berichtigt hat. Mit Ostien, die durch zungenförmige Ǩlappen

1) ANm. Eine Artdiagnose kann bei dem einzigen Vertreter einer neuen Gattung wohl füglich unterbleiben; ich stimme hierin völlig L. S. Schultze bei (1SgS, p. 447).

SIBOGA-EXPEDITIE XI. 
zweigeteilt werden, wodurch ebenfalls eine $\$$-Zahl vorgetäuscht werden kann, sind die vorliegenden Bildungen nicht zu verwechseln.

Die Arme gehen als 8 massige, walzige Gebilde von dieser Scheibe ab, bei jüngeren Tieren deutlich paarweise genähert; bei ältern gleicht sich dies jedoch aus. Zwischen den Oberarmen entstehen so 8 bogenfenstrige Ausschnitte (Fig. 47); durch diese treten an ältern Tieren die sehr entwickelten Axialkrausen mit ihren Seitenläppchen und langen Filamenten nach aussen heraus, was der ganzen Meduse einen sehr eigentümlichen Habitus verleiht. Die Basis des Fensters bezeichnet den Anfang der Unterarme, die von etwa gleicher Länge wie der Oberarm und pyramidenförmig sind. Sie zeigen zwei tief eingeschnittene Flügel, die aber so dicht, beinahe federnd, an einanderliegen, dass es einiger Gewalt bei älteren Tieren bedarf, sie zum Auseinanderklappen zu bringen. In normalem zugeklappten Zustand erscheinen sie so als die Aussenfläche der Unterarmpyramide, und nur eine Rinne (Fig. 47 und 73 x) zeigt den Einschnitt zwischen den Flügeln an. Die Seitenflïchen der Unterarmpyramide sind von Krausen grösstenteils frei, da diese besonders bei jüngeren Tieren auf die Kanten beschränkt sind und erst am distalen Ende auf die Fläche heribergreifen. Die Krausen sitzen nicht direkt auf, sondern auf kleinen Stielen, die im Lauf der Entwicklung zu ansehnlichen Seitenästen werden, sodass man auch hier von einer secundären Fiederung der Arme, oder vielmehr der Flügel reden könnte. Durch reiche Entfaltung der Krausen treten aber diese A'este ganz zuriick, und man erkennt, wie bei Crambessa erwähnt (5. O. p. 47) namentlich von der abaxialen Seite aus, nur einen dichten wolligen Besatz. Wir sehen also auch hier, wie bei Crambessa, dass eine typisch-dreifliglige Form der Arme mit zunehmendem Alter und bei zusammengeklappten Unterarmfligeln sich als dreikantige Form mit scheinbar allseitigem Krausenbesatz präsentirt, dass also die Unterscheidung von tripter und trigon nur eine künstliche ist. Die Canalversorgung der Arme folgt der äusseren Verzweigung; ein axialer Hauptcanal giebt, den ventralen Seitenästchen entsprechend, schon am Oberarm Seitencanälchen rechtwinklig ab; an der Flügelstelle teilt er sich in zwei Schenkel für die Abaxialfligel, die sich sofort weiter spalten, und in einen zurïckbleibenden diinneren Stammast für die ventralen Unterarmkrausen, der sich aber ebenfalls fast sofort auflöst, so dass seine Aeste, wie für Himantostoma beschrieben, noch etwas parallel laufen, ehe sie zu ihren Krausen einbiegen.

Von Armanhängen sind erstens die unscheinbareren Kölbchen zu erwähnen, die uiber die Saugkrausen nicht hervorragen und sich (an den grösseren Exemplaren) nur durch ihre rötliche Farbe aus der weisslichen Krausenwolle herausheben. Sie sind uberall zerstrent und wie bei Vetrostoma kurz gestielte Gebilde mit dichtem Nesselbesatz am Ende. Teilweise sind die Nesselwärzchen wieder selbstständige, abgesetzte Fäden. Der Canal in ihnen ist zuerst sehr verengert, zeigt dann eine dem dicken Ende entsprechende Erweiterung und endigt blind. Zweitens sind die langen und starken Peitschenfilamente für die Form charakteristisch. Bei jungen Exemplaren zeigen sie eine gewisse Regelmässigkeit der Verteilung; besonders starke Peitschen stehen je eine an den 4 Hauptgabelstellen der Arme im Centrum und andre ansehnliche in Abständen auf der axialen Krausenreihe; ebenso zeigt jeder der abaxialen Flügel ein Filament. Mit zunehmender Grösse der Meduse vermehren sie sich jedoch sehr stark und bilden manchmal ganz unregelmässig da und dort angehäufte Bündel, die zumal aus den Armfenstern charakteristisch herausragen. Im Inneren besitzen sie wie die Kolben einen entodermalen Kanal, der jedoch 
gleichmässig dünn bis ans blinde Ende verläuft; auf ihrer Aussenseite sind sie mit länglichen Nesselwarzen dicht besetzt.

Der Centralmagen zeigt die typische, ausgeschweifte Ḱreuzform; die pcradialen Enden der Kreuzschenkel sind etwas breiter wie die Mittelstelle und herzförmig ausgeschnitten. Eigentumlich dagegen erweist sich das $\mathrm{C}$ a nalsystem, sowohl durch die Art der Verzweigung als durch Schärfe der Begrenzung. Es gehen vom Magen 8 rhopalare Radiärcanäle aus und ausserdem noch 8 adradiale, den perradialen mehr genäherte (Fig. 47 und 50); alle 16 treffen jedoch den Ringcanal in gleichen Abständen; die adradialen endigen hier, die perradialen und interradialen lassen sich noch bis zum Beginn der Rhopalarlappen verfolgen (Fig. 4S). Zwischen den 16 Radiärcanảlen liegt nach innen vom Ringcanal ein einfaches weitmaschiges Netz, das nicht mit den Radiärcanälen anastomosiert, wie bei Crambessa, sondern nur durch den Ringcanal mit diesen an der Basis zusammenhängt; dieses Netz reicht nur eine kurze Strecke, $3-4$ Maschen weit centripetalwärts, sodass eine grosser Raum der Subumbrella in diesen 16 Vierecken von Canalsystem völlig frei bleibt (Fig. $48,49,50$ ). Dadurch ist auch ein wesentlicher Unterschied von dem Arcadennetz der scapulata (z.B. Rhizostoma) gegeben, das sehr weit centralwärts reicht. Auch bei den erwachsenen 25 und $38 \mathrm{~cm}$. grossen Stücken, hat dieses Netz i. V. keine grössere Ausdehnung und die Maschenzahl ist ungefähr die gleiche; ebenso wenig haben sich die radiären Canäle vermehrt. Der Ringcanal zeichnet sich durch scharfe Begrenzung vor andren tripteren Formen aus. Ausserhalb des Ringcanals findet sich ein viel engeres Canalnetz, in welchem sich die $\&$ Rhopalarcanäle durch Caliber etwas auszeichnen, mit dem sie sonst aber durch zahlreiche Anastomosen verbunden sind. In die beiden Rhopalarlappen gehen zwei verhältnismässig breite, unverzweigte Canäle, was gegenüber dem übrigen Netz sehr auffällt. Am Schirmrand folgen die Netzmaschen nicht der Lappenform wie z.B. bei Mastigias (s. Fig. 58) sondern das ganze Netz schneidet mit einer ziemlich ganzrandigen Bogenlinie ab, wenn man will, einem äussern kleineren Ringcanal. Den Zwischenräumen zwischen den einzelnen Lappen entsprechen radiär verlaufende Canalstückchen, die sich durch ihren gestreckten Weg etwas aus dem übrigen Netzwerk herausheben (Fig. 48 can $r$ !); durch Caliber sind sie aber, ebensowenig wie der den Randabschluss bildende Canal vor den ibrigen Naschen ausgezeichnet. Der Randabschluss zeigt nur ganz seichte Einbuchtungen, entsprechend den Lappen.

Die Muskulatur (s. Fig. 48) zeichnet sich durch grosse Einfachheit aus. Eigentlich kann man nur von einem einzigen starken Ringmuskel sprechen. Innerhalb des Ringcanals sind die Züge so dünn und zerstreut, dass sie übersehen werden können, ausserhalb desselben schliessen sie sich jedoch fest zusammen und haben ansehnliche Stützleisten, so dass an den grossen Exemplaren ein beinahe fleischiger Ring, sogar von der. Exumbrella aus, zu erkennen ist. In den 8 Radien sieht man zwar die Rhopalarcanäle durchscheinen, aber es findet nicht, wie bei Mastigias und A., eine Unterbrechung der Muskulatur statt, sondern die circulären Muskelfasern verlaufen auch hier weiter. Am Schirmrand gehen die Fasern etwa so weit wie die Canalverzweigungen und lassen die peripheren Enden der Lappen frei.

Die Randlappen sind nach Form und Zahl bei jüngeren und älteren lixemplaren etwas verschieden. An den jüngsten Tieren sind es nur kleinere halbmondförmige Hervoragungen, die am Schirmrand wenig nach aufwärts reichen. Ihre Zahl beträgt meistens $\&$ pro Octant, und 
es gehören je 2 zu einem grösseren Lappen zusammen, aus dem sie durch Teilung entstanden sind. Wenn eine unregelmässige Zahl und ungleiche Grösse zu bemerken ist, so kann das in vielen Fällen auf noch nicht ausgefuhrte Lappenverdoppelung zurïckgefiihrt werden. Auch an den grössten wird die S-Zahl nur wenig überschritten; alle \& Lappen reichen dann mit ihrer Einkerbung hoch am Schirmrand hinauf und bilden so rechteckige, nach unten abgerundete Platten; einzelne von ihnen sind weiter geteilt, so dass die ro Zahl pro Octant sehr häufig ist. Gegeniber diesen Velarlappen zeichnen sich die Rhopalarlappen, wie schon erwähnt, durch ihre ungeteilten Canäle (s. Fig. 48 rh) aus, ferner durch die spitze Form und das Convergieren nach oben, das sich auch noch den benachbarten Velarlappen mitteilt (Fig. 53). An Grösse stehen die Rhopalarlappen hinter den Velarlappen beträchtlich zurück und zwar um so mehr je älter die Tiere sind. An ausgewachsenen Exemplaren beträgt ihre Länge kaum $1 / 3$ von der der Velarlappen.

Die R hopalien selbst sind gestreckte Kölbchen mit bulböser Anschwellung und paarigen P'igmentflecken. Sehr auffallend wie bei Crambessa, ist die äussere Sinnesgrube (Fig. 53 fos. ol.) von assförmiger Gestalt mit radiären, am Kolbengrund zusammenlaufenden Rinnen und Falten. Ectodermale Differenzierung dorsal auf dem Otolithensack und ventral auf dem Bulbus, treten ebenfalls schon im Aufsichtsbild hervor (Fig. IOO). Merkwurdig sind die paarigen Augenbildungen, die ausser dem schmalen Pigmentfleck mit diesem und der ectodermalen Differenzierung zusammenhängend an den Seiten sitzen. Noch besser treten sie an genau radial, etwas seitlich von der Medianen geführten Längsschnitten hervor (Fig. IO4 oc). Die grubenförmige, stark pigmentierte Einsenkung trennt dann scheinbar den entodermalen Canal des Kolbens vom Endsack; genau median hängen Entoderm und Concrementzellen zusammen. Die ectodermalen Differenzierungen sind sehr stark entwickelt, und ein sehr hohes Nervenepithel (ect!) ist an den bewussten Zellen des Kolbens zu erkenıen. In der äusseren Sinnesgrube kommen auch noch Drüsenzellen dazu. Gerade diese letzteren sind es, die durch ihre Anordnung die Zeichnung des Aufsichtsbildes bedingen. Die Verhältnisse sind ähnlich von Crambessa mosaica durch LENDENFELd beschrieben worden.

Die Gonaden zeigen die typische Gestalt und Lagebeziehung. Sie bilden ein engeres Kreuz wie die Magenhöhlung selbst; dessen 4 interradiale Schenkel kommen im Perradius so nahe zusammen, dass die Figur fast einheitlich wird. Den verhältnismässig engen Subgenitalostien nach wirde man nicht denken, dass sie in einen einheitlichen Hohlraum führen; doch kann man schon mit einer Borste zum einen Ostium herein- und zum benachbarten herausgelangen, und Medianschnitte durch die ganze Meduse zeigen, dass die perradialen Pfeiler nur einen geringen Raum einnehmen, und der Subgenitalsaal nach innen, auch im Centrum, gänzlich frei und ungeteilt ist. Eine Brutpflege, wie bei Rhizostomen die Regel, wurde auch hier bei einigen Individuen beobachtet. Zwei Exemplare von Stat. 209 zeigen sich dicht erfüllt mit Planulae, die teilweise auf den Armen zwischen den Saugkrausen, und teilweise auf der Subumbrella, besonders in deren peripherem Teil in allen Grössen zu finden sind; an letzterer sitzen sie jedoch nicht frei, sondern in besondere Falten der Gallerte eingebettet, wie dies ebenfalls von mehreren Acraspeden beschrieben ist. Die betreffenden Exemplare sind beträchtlich kleiner wie die Riesenexemplare; man könnte also sich die Frage vorlegen, ob es sich um dieselbe Art handelt, zumal auch der Habitus ein wenig anders ist. Doch sind sie nach meiner speziellen Untersuchung in allen Verhältnissen der Organisation, den Armen, Armanhängen, der Canal- 
verzweigung etc. bis in die kleinsten Einzelheiten dem typischen Formen so genau gleich, dass an einer Identität kein Zweifel sein kann. Der veränderte Habitus rührt nur daher, dass sie durch die Geschlechtsreife, Brutpflege etwas angegriffen, struppirt sind; die Armkrausen teilweise defect werden, und sich, wie an solchen Tieren gewöhnlich, Zeichen des Eingehens bemerkbar machen. Man darf also annehmen - und hierfuir sprechen auch Erfahrungen an andern Medusen - dass das Eintreten der Geschlechtsreife wohl von einem gewissen Stadium ab, aber nicht an ein ganz bestimmtes Grössenstadium gebunden stattfindet, und eventuell durch äussere Umstände verfrüht, resp. verzögert werden kann.

Bei der mit Crambione nächstverwandten Gattung Crambessa, die sich durch das Fehlen jeglicher Armanhänge unterscheidet, aber im Canalsystem ihr nähert und sich dadurch von den Gattungen Loborhiza und Toxoclytus abgrenzt, die gleichfalls keine Armanhänge tragen, ist auch diese Configuration des Canalsystems in die Diagnose aufzunehmen, und die Arten sind darauf hin zu revidiren. Cr. palmipes, bei der L. S. Schultze die Verschiedenheit der Canalverzweigung zuerst betont hat, muss darum ausscheiden; diese Form ist auch noch durch andere Eigentumlichkeiten, verwachsene Oberarme, Mundfilamente (I \&98, p. 454) ausgezeichnet, und dürfte ein eignes Genus erfordern. Für die typischen Crambessa-formen sind 8 Rhopalarcanäle und 8 nur bis zum Ringcanal reichende Adradialcanäle, ein engmaschiges extracirculäres Netz und ein weitmaschiges, kurzes und blindes intracirculäres Netz charakteristisch; wie hier bei Crambione, nur dass dieses blinde Netz bei Crambessa auch mit den adradialen Canälen in Verbundung steht (s. Schultze I 897, p. I6I) und Grenacher i 876 (Fig. I3), hier dagegen nur mit dem Ringcanal. An dieser Stelle möchte ich darauf hinweisen, dass auch Mastigias orsini ein ganz ähnliches Canalnetz hat und sich dadurch von allen andern Angehörigen der Gattung Mastigias unterscheidet; zudem sind bei letzterer Art auch die Endknöpfe von den typischen Mastigias-Kolben verschieden und eigentlich nur kurze, krausenfreie Teile des Unterarms. Da auch solche freien gallertigen Endteile bei Crambessa vorkommen, z.B. Cr. Stuhlmanni Chun ( 896, p. I 2), so läge die Vermutung nahe, $M$. orsini hier in die Nähe einzuordnen. Bei Cr. Stuhlmanni tritt der krausenfreie Teil allerdings nur an der Abaxialseite hervor (1.c. Fig. I), während ,die axiale Saugkrause sich bis zur Spitze fortsetzt"; doch stellt dies nur einen Uebergang zum Verhalten von orsini dar. Ein positiver Entscheid ist einstweilen nicht zu fällen, nur der negative, dass wie $C r$. palmipes aus Crambessa, so $\boldsymbol{M}$. orsini aus Mastigias auszuscheiden ist. Auch die Pscudorhiza-Arten sind darum, wenn auch Mastigias sehr nahe stehend, doch nicht generisch damit zu vereinen, da sie wie übereinstimmend beschrieben wird, 8 rhopalare, 8 adradiale Canäle besitzen und anstatt des inneren weitmaschigen Netzes eine Anzahl gestreckter blindendender Centripetalcanäle aufweisen, nur für Ps. Thocambayi von Agassiz und Maver (I 899) ist die Canalverzweigung nach der Beschreibung und Figur fraglich.

Für die noch übrigen Gattungen Toxoclytus und Loborliza, deren Arme ebenfalls ohne Anhänge sind, kämen ebenfalls noch Unterschiede im Canalsystem in Betracht. Gerade $T$. rosens, für welche Agassiz die Gattung aufgestellt hat, besitzt nach der Originalabbildung von Lesson Gallertknöpfe und wird darum von VAnHöffen zu Mastigias gestellt; die Art 
tripterus zu Crambessa, und die Gattung gestrichen. L. S. Schultze hat aber unter Aufstellung ciner neuen Art $T$. turgescens die Gattung ungefähr mit der HaEckeL'schen Diagnose aufrecht erhalten; VAxmörfex hält es jedoch neuerdings für „nicht unwahrscheinlich”, dass sowohl turgescens wie tripterus dreiflighlige Mundarme besitzen und zu seiner Gattung Lobor/hiza gehören. Die Aehnlichkeit der Armform scheint auch mir sehr gross; auch erwähnt Schultze, dass die Saugkrausenästchen fiederspaltig sind, was auf die Verhältnisse von Loborhiza zuträfe. Das Canalsystem ist indessen sehr deutlich unterschieden; bei Loborhiza Vanhöffen (I8S8, p. 28) ein allgemeines Anastomosennetz, das mit dem Magen zusammenhängt, ohne deutlichen Ringcanal. Bei Toxoclytus laut Schurtze (bei turgescens, bei den andern spec. unbekannt) ein Ringcanal, ein äusseres enges Maschennetz, und ein inneres weites, den Magen nanscheinend nicht erreichendes weitmaschiges Gefässnetz" (I \$98, p. 456), dazwischen weitere unverzweigte Radiär- und Centripetalcanäle. Einzelne centrale Filamente auf den Armscheibe werden erwähnt, indess sie auf den Armen fehlen. Es dürfte daher einstweilen die Gattung erhalten bleiben; grade im Canalsystem mit den blinden Enden zeigen sich Hinneigungen zu Lychnorhiza und Pscudorhiza, während sich Loborhisa hierin mehr Mastigias und den Himatostomiden nähert.

Crossostoma L. Ag. IS62.

Stomaster L. Ag. I 862.

Crossostoma Haeckel is79.

Crossostoma Sens. emend. Vanhöfen iss8.

Rhizostome mit dreifligligen Mundamen, die secundäre, tief eingeschnittene Lappen zeiger, an denen die eigentlichen Krausen sitzen. Mit keulenförmigen Blasen besonders an den beiden abaxialen Flügeln (und peitschenartigen Anhängen an dem axialen Flügel und im Centrum). Mit einer aus isolierten Saugkrausenstücken bestehenden (und mit Filamenten untermischten) Mundrosette. Canalsystem mit 8 Radiär-und zahlreichen Zwischencanälen, so dass ein schon vom Magen ausgehendes dichtes Anastomosennetz entsteht. Mit circulärer in den $S$ Radien unterbrochener Muskulatur. Rhopalien mit einfacher Sinnesgrube, ohne Ocellus.

Es ist fraglich, ob mit der obigen in Anlehnung an VAxnöfFEN gegebenen Diagnose die Gattung nicht einen andern Sinn bekommen hat, als die Vorgänger bis HAECKEL ihr zuschreiben wollten, besonders da sie eigentlich nur auf Grund der sehr unvollkommenen Beschreibung und Abbildung von Tilesius ( I 83 I) von Agassiz ( I 862) aufgestellt und von HaeckeL schon sehr modifiziert worden ist. Tilesius hatte die betreffende Arten in ihrer Armverzweigung als Cassiopeien aufgefasst, derselben Meinung, in der Deutung der Arme scheint AGassiz gewesen zu sein; nur hat er auf Grund der centralen Rosette besondere Gattungen, Crossostoma und Stomaster geschaffen, die HAEckel zu e in er zusammenzieht. HaEckel bringt die Gattung in nächster Nähe seiner Versura unter; diese zeigt aber, laut seiner eigenen Abbildung, deutlich dreifliglige Arme, die nur secundär tief gelappt sind und deshalb eine Fiederung vortäuschen. VAnHöfFex hat deshalb mit Recht Versura zu den tripteren Formen, bei denen (s. z.B. HaEckel's 
Lychnorhiza) auch eine secundäre Lappung vorkommt (also nicht als unicrispe) gerechnet, und ebenso Crossostoma, die sich laut HAECKEL selbst nur durch die centrale Zottenrosette von Versura unterscheidet. Es ist hier allerdings ein Fall gegeben, wo sich wie bei Mastigias laut L. S. Schultze (i 898, p. $45_{4}$ ) schwer unterscheiden lässt, ob man eine unicrispe oder multicrispe Armform vor sich hat. Einerseits lassen sich drei Flügel unterscheiden und die Aestchen als secundärer Zerfall auffassen, andrerseits ordnen sich die Gallertäste serial und gewinnen eine solche Selbstständigkeit, dass man von Fiederung, Trichotomie reden kann. Es ist aber immerhin die dreifluglige Form bei Versura so ausgeprägt, dass ich kein Bedenken trage, hierin VANHÖFfEN zu folgen, um so mehr als auch andere dreiflüglige Formen einen solchen Zerfall der Flügel in einzelne Aeste zeigen und insofern als dieser Zerfall oft nur einzelne Aestchen betrifft, der übrige Flügel ganz bleiben kann. Ich fasse also die Dreiflügligkeit hier als das primäre Verhalten auf. Einen Unterschied zwischen tripteren und trigonen Formen, wie VAxhöffes, braucht man auch hier nicht zu machen, sondern hat, wie a. a. Stelle (s. o. p. 46) erörtert, den Begriff dreiflüglig im gewöhnlichen weiteren Sinn aufzufassen. Ueber weitere Ableitungsmöglichkeiten, zu denen die Armform hier Anlass giebt, wird noch bei der näheren Schilderung der Arme, über andere noch hier eventuell einzubeziehenden Gattungen noch am Schluss der ganzen Beschreibung zu reden sein.

Von drei bei HAEckel aufgefuhrten Arten ist es gerade bei der Stammart der Cassiopin frondosa Tilesius, die Agassiz zur Grindung der Gattung Crossostoma veranlasst hat, mir sehr fraglich, ob damit eine Crossostoma nach HaECKEL und nach VANHöfFen und nicht eine Cassiopcia auch im heutigen Sinn, gremeint ist. Mir scheint das Tilesius'sche Bild der Arme eher auf eine Verzweigung wie bei Cassiopcia zu deuten, und die Rosette keine besondere Bildung, sondern nur durch die zahlreichen, der Brutpflege dienenden, centralen Gallertblättchen veranlasst zu sein, die auch ron HAECKEL bei Cassiopeia, wenn auch mit falscher Deutung, abgebildet wird ( 1879 , Taf. XXXVII, Fig. 5), und die ich oben bei Cassiopeia andromeda des näheren beschrieben und erläutert habe (p. 42 und Fig. 29 ko c). Bei der zweiten Art, Crossostoma corolliflor $\alpha$ (canariensis) ist es eher möglich, dass sie dem HAECKEL'schen und auch dem modifizierten VANiöFfEn'schen Crossostoma-Begriff entspricht; hier ist der centrale Stern auch in der Tilesius'schen Darstellung eine eigenartige Bildung, und die Armverzweigung eher auf die oben erörterten Verhältnisse deutbar; auch hat HAECKEL hiervon ein wirkliches Exemplar vor sich gehabt, allerdings "teilweise zerstört und nicht gut conserviert", an dem sich einerseits die Uebereinstimmung mit Vorsura im Magen und Subgenitalporticus, anárerseits die Trennung wegen der Zottenrosette erkennen liess. Da aber gerade diese Art, von der die Gattungscharactere abgeleitet werden können, bei Agassiz nicht Crossostoma, sondern Stomaster benannt ist, so ist es schwer zu entscheiden, welcher Namen den Vorrang haben soll. HAEckel hat die Genera zusammengezogen, weil Agassiz keinen generischen Unterschied angegeben habe; dieser Vorwurf ist unberechtigt, denn Agassiz schreibt (1862, p. I54), dass bei St. die Rosette doppelt ist und die Genitaltaschen sich nicht am Rand teilen (wie bei Crossostoma). Dennoch glaube ich, dass man die Bezeichnung Crossostoma von HAECkEL wählen soll, weil dieser zum ersten Mal eine wirklich kenntlich machende und die Möglichkeit der Wiederauffindung bietende Diagnose damit 
verbunden hat, einerlei ob die TiLesius'sche unvollkommene Darstellung damit gedeckt ist oder nicht. Sonst ist die Grefahr vorhanden, dass immer wieder neue Gattungen aufgestellt werden und andere alte sich als blosse Phantome in der Litteratur fortschleppen, ohne jemals in Fleisch und Blut - resp. in Gallerte und Zellen — gesehen zu worden.

Es wäre aus denselben Grinden auch möglichst zu vermeiden, eine neue Spezies hier zu machen. Goette (1886) hat eine Crossostoma des Sanders'schen Materials nicht neu benannt, weil die „Exemplare möglicherweise zu einer der drei bisher aufgestellten Arten ... gehören, was aber bei der unvollkommenen Beschreibung derselben noch nicht zu entscheiden ist". Mit dem GoETtE'schen Stick $a$, dieser unbenannten Species das ich im Original vergleichen konnte, ist die vorliegende Form identisch, ohne dass ich sie auf eine der HAFckel'schen beziehen könnte. Die dritte der Haeckel'schen Arten Cr. Dubreuillii, die Agassiz zu Toxoclytus und Haeckel selbst nur mit doppeltem Fragezeichen hierhergestellt hat, ist von VANHöffEN auf Grund der Originalabbildung bei Lesson ( I $8_{30}$ ) zu Crambessa gezogen werden; die zweite Art Cr. frondifora (frondosa) ist aus den oben angefihhrten Grinden sehr problematisch. Von der ersten, $C r$. corolliflora (canariensis) sind, wie aus der untenstehenden Diagnose und Beschreibung hervorgeht, merkliche Unterschiede vorhanden, wenn man die ungenügende Beschreibung bei TiLEsius und HAECKEL iberhaupt anerkennt. Die nachfolgende Beschreibung soll daher nicht nur der Artkennzeichnung, sondern auch der von HAECKEL sebst wiederholt gewuinschten (I879, p. 608) Festlegung der Gattung dienen.

I. Crossostoma Anadyomene n. sp. (Taf. VII, Fig. 65, 66, 67, 68).

Crossostoma species nova? Goette is86.

Stat. 3I4. I Exemplar von etwa $20 \mathrm{~cm}$. Schirmdurchmesser.

Der Schirm ist wenig gewölbt; die Exumbrella zeigt eine auffallende Structurirung und Zeichnung, dadurch dass ihre ganze Oberfäche von anastomisierenden Rinnen durchzogen wird, zwischen denen unregelmässige, erhabene Polygone übrig bleiben. Das ganze Netz ist wie das Canalsystem auf der Subumbrella im Centrum weitmaschiger, an der Peripherie enger und hat eine radiäre Verlaufstendenz. Bei der Dünne der Schirmgallerte könnte man daran denken, dass es durch das Durchscheinen des Canalsystems zu Stande käme, doch zeigt sich deutlich, wenn man bestimmte engmaschige Teile des letzteren im Auge behält und dann an der Oberfläche wiederfinden will, dass dies nicht zutrifft. Auch ist die exumbrellare Structur im Centrum über der Magengegend ebenfalls ausgeprägt, und erweist sich schon dadurch wie durch die Vertiefung als eine selbstständige Exumbrellarstructur. Auch durch intensivere Färbung scheinen die Rinnen, soviel an dem Exemplar noch kenntlich ist, gegenüber der ubbrigen Exumbrella sich auszuzeichnen. Die Gallerte ist auffallend diinn, sodass der Schirm selbst in der Mitte nur wenige Millimeter stark wird, und das ganze Tier dadurch einen schlaffen Habitus erhält, anch in den blattartig dünnen Unterarmen mit ihren Seitenästen. Die einzigen Stellen stärkerer Gallertentwicklung sind die Unter- resp. Aussenseite der Armscheibe und die sich daran ansetzenden Teile der Oberarme in ihrem abaxialen Teil. 
Die Arme selbst sind sehr stark seitlich comprimirt, der Oberarm hat dadurch die Form eines Messerstücks; die Schneide entspricht der axialen Seite; der Rücken dem etwas rundlicheren und massiveren Abaxialteil (Fig. 66). Die Seitenflächen bilden einen Rhombus, dessen obere Kante sich von der Armscheibe scharf absetzt (Fig. 66 br) und von dessen unterer, etwas längerer Kante man den Beginn des Unterarms resp. der Dreiflügligkeit rechnen kann. Der ganze untere Teil des Arms zeigt, ähnlich wie für Lychnorhiza von HAECKEL beschrieben wird, seitlich abgehende Aeste, die wie der Arm selbst durch die Düne der Gallerte nur in die Fläche, beinahe membranös, entwickelt sind. Diese Aeste verteilen sich auf die 3 Flügel; von manchen ist es jedoch zweifelhaft, ob man sie zu den Seitenflügeln oder zum axialen Flügel rechnen soll, und insofern ist die Reinheit der tripteren Verzweigung getrubt. Alle diese abgeplatteten membranösen Seitenäste sitzen mit breiter Basis an, verschmälern sich darauf etwas und breiten sich dann wieder fächerförmig aus (Fig. 66 z!). Der Kreisbogen des Fächers bezeichnet die Ansatzstelle der Saugkrausen. Diese sitzen also an den eigentlichen Aestchen nur ventral, und man könnte mit einem gewissen Recht, von der tripteren Hauptverzweigung absehend, von einer unicrispen Armform reden. Auch hierin haben wir somit eine vermittelnde Form der Krausenverteilung vor uns, und in der That ist eine gewisse Aehnlichkeit mit dem Bild von Cotylorhiza (Fig. 69) nicht zu verkennen. Wenn man sich deren gleichmässige Unterarmcurve gerade an der durch den grossen Kolben markirten Stelle scharf geknickt denkt und gleichzeitig in der stüzenden Gallertmasse der Arme entsprechende Wachstumsverschiebungen annimmt, so entstelien aus den herumgebogenen Sticken des dichotomen Unterarms die beiden abaxialen Flügel der tripteren Form. Noch grössere Aehnlichkeit haben Armbildungen, wie bei Netrostoma, wo die Umkrümmung sehr weit geht und deutliche Seitenisiste, allerdings nicht platt wie hier, zu erkennen sind (Fig. $3 \xi$ ).

Sehr eigentümlich und nicht ohne Beziehung zur morphologischen Ableitung ist hier die Canalversorgung der Arme. Ein grosser Hauptstamm verläuft der abaxialen Kante genähert und parallel (Fig. 66 und 67). An der Fligelstelle teilt er sich entsprechend den axialen Flügeln und giebt, allmählich dünner werdend, die Aeste für die Seitenblättchen ab. (An der Figur konnte natürlich nur die Verästelung für den einen, dem Beschauer zunächst liegenden Flügel eingezeichnet werden). Die gesammte axiale Kante mit ihren Blättchen wird jedoch nicht, wie man sonst von tripteren Formen erwarten sollte von ventralen Aesten dieses Hauptcanals versorgt, sondern ders̉elbe bleibt bis zur erwähnten Teilungsstelle gänzlich unverzweigt, und für die ventralen Blättchen verläuft ein eigener schwächerer ventraler Canal (Fig. 66) dem ersten parallel im Oberarm. Derselbe erschöpft sich jedoch damit nicht, sondern geht merkwürdigerweise noch weiter und beteiligt sich auch noch an der Versorgung der abaxialen Krrausen (Fig. 66 und 67), so dass jeder Flügel mit seinen seitlichen Aesten ein zierliches paralleles Doppelgeflecht von Gefässen erhält, das durch die membranöse Gallerte deutlich durchschimmert. Die Gefässe gehen ohne mit einander zu anastomosieren bis in die letzten Verzweigungen und teilen sich so in die Versorgung der Saugmündchen. Auch hierdurch ist also eine Mischung von uni- und multicrisper Saugkrausenverteilung angedeutet.

Die kolbenförmigen Anhänge vermehren noch die Aehnlichkeit mit der dichotomen Cotylortiza. Sie entsprechen in Gestalt und Verteilung genau der Abbildung die HaEckel SHBOGA-EXPEDITIF, XY. 
für die nahe verwandte Vorsura giebt (1879, Taf. 40, Fig. 9). Die meisten sind gedrungen und kurzstielig, andere wenige länger und gleichmässig walzig. An der axialen Kante scheinen sie fast zu fehlen; an den abaxialen Flügeln sind sie dagegen sehr reichlich und unregelmässig verteilt. Eine besondere Bevorzugung der Armspitze ist nicht wahrzunehmen. Zahlreiche kleinere Kölbchen sitzen noch ausserdem zwischen den abaxialen Saugkrausen, ohne über dieselben hervorzuragen, und machen sich nur durch die intensivere Orangefärbung bemerkbar, die ihnen, ebenso wie den grossen Kolben zukommt. An der axialen Seite werden die Kolben durch die walzigen Anhänge vertreten; nach oben werden diese zahlreicher aber immer dünner, so dass man schliesslich von richtigen Peitschenfilamenten reden kann. Auch das GoEtTE'sche Exemplar trägt solche Fäden.

Die Mundrosette kommt dadurch zu Stande, dass die $2 \times 4$ Krausenkanten der Oberarme nicht in den bekannten Kreuzlinien im Centrum zusammenlaufen, sondern dass sich hier eine Anzahl isolirter Saugkrausenstücke, ebenfalls auf besondern Blättchen stehend, wie an den Armen, finden. Dies ist um so bemerkenswerter, als die Blättchen an dem Oberarm selbst in seinem oberen Teil aufhören und die Saugkrausen direkt seiner axialen Kante ansitzen; (Fig. 66). Würde sich dies Verhalten bis ins Centrum fortsetzen, so käme die normale Kreuzfigur zu stande, wie sie laut HaEckel bei $V_{\text {crsura }}$ besteht. Anstatt dessen sitzen aber hier wieder kleinere fächerförmige Gallertläppchen (Fig. $66 \mathrm{kr}$ c) mit Krausen, die mit Peitschenfilamenten untermischt, von der Fläche gesehen, die bewusste Rosette darstellen (Fig. 68). Mit dem aus platten Kolben grebildeten und der Brutpflege dienenden Mittelfeld der Cassiopeien (Taf. IV, Fig. 29), ist diese Bildung nicht zu verwechseln; sie steht vielmehr hier in Zusammenhang mit der Verästelungstendenz der Mundarme. Auffällig bleibt darum um so mehr, dass sie bei Versura, wo doch die gleiche Verästelungsart der Arme besteht, fehit.

Die Armscheibe mit den weit offenen Subgenitalostien und den schmäleren abgerundeten Pfeilern, gleicht der der Crambessiden und Himantostomiden, mehr noch als es HAECKEL von Versura abbildet. Die weiten schlitzförmigen Ostien sind durch keinerlei Klappenbildung verengert. Der Subgenitalsinus selbst ist ein niedriger, weiter Raum, an dessen Dach die Subumbrella einen wesentlichen Anteil in den Interradien hat. Die Gonaden waren an dem vorliegenden Exemplar entleert und nur ihre Ansatznähte noch wahrzunehmen (Fig. 65 gon.). Das Gastrogenitalkreuz zeichnet sich durch ausserordentlich verschmälerte Gestalt aus; die 4 perradialen Schenkel des Magens sind sehr gestreckt, jeder etwa 6 mal länger als die Stelle ihres Zusammentreffens. Dadurch dringt in den 4 Interradien die Subumbrella mit den Anastomosen des Canalnetzes ausserordentlich weit centralwärts ein, und das Magenkreuz zeigt eine scharfe, fast eckige Gestalt (Fig. 65), wie sie in diesem Grad von keiner anderen Rhizostomee, auch unter den Himantostomiden nicht, erreicht wird.

Von den 8 rhopalaren Canälen, die deutlich bis zum Rhopalium selbst durch radiären Verlauf und Caliber erkennbar sind (Fig. 65 can rh), gehen die 4 perradialen direkt vom Magen, vom Ende der Kreuzesschenkel aus; die 4 interradialen dagegen heben sich erst eine beträchtliche Strecke vom Magengrund entfernt aus dem Anastomosennetz heraus, durch das sie mit dem Magen und den übrigen zahlreichen Zwischencanälen verbunden sind. Dieser innere interradiale Teil des Anastomennetzes ist offenbar secundärer Entstehung; anders ist der scharf radiale und 
doch erst in der Mitte beginnende Verlauf des Interradialcanals nicht zu erklären. Der Magen war zuerst nicht so eingeengt, hat auch interradial weiter gereicht; seine Kreuzform war nicht so extrem gestreckt; erst nachträglich haben dann weitere Verlötungen im Entoderm stattgefunden. Es zeigt sich das auch darin, dass dies interradiale Netz zwischen Magenecke und Canal viel unregelmässiger und weitmaschiger ist, als die adradialen Netzteile, die von nebeneinander aus dem Magen entspringenden Canälen ausgehen. Nach der Peripherie zu werden die Maschen des gesammten Netzes enger, auch treten sie in Verbindung mit den 8 Hauptcanälen. Ein besonderer Ringcanal ist nicht zu erkennen. Am Schirmrand bilden sich den Lappen entsprechend besondere Geflechte, die bis fast zum Rand der Lappen hin verfolgbar sind.

Die Muskulatur der Subumbrella zeigt keine Radialzüge, sondern nur circulären Verlauf. Man kann, wenn man will, einen äusseren Ring (Fig. 65 mu cir), der bis in die Basen der Randlappen reicht, unterscheiden von einer inneren, unterbrochenen Zone. Dass beide besondere Krummungen gegeneinander zeigen, wie es HaEckel für Versura zeichnet (I879, Taf. 40, Fig.9), konnte ich nicht wahrnehmen. In den 8 Radien findet eine merkliche Verduinnung und teilweise Unterbrechung der Muskelzigge statt; ausserdem reichen im Interradius die Züge viel weiter centralwärts wie im Perradius, wo sie noch weit vom Pfeiler entfernt, abschneiden. Es kommt dadurch die charakteristische Figur von 4 interradialen, halbirten Sectoren zu Stande, oder besser gesagt, von $\&$ adradialen, fast rechtwinkligen Dreiecken, die mit kleineren Katheten aneinanderliegen, und deren Spitze, nach dem Centrum zuliegend, abgestumpft ist.

Der Schirmrand zeigt zwischen je zwei Rhopalien 8 grosse, halbkreisförmig abgerundete Lappen. Dazwischen sind kleinere und schmälere, mehr zungenförmige Läppchen eingeschoben, nicht so regelmässig wie die 8 grösseren. Der äusserste Rand der Lappen besteht nur aus zwei ectodermalen, durch eine dünne Stützlamelle getrennten Epithellagen; ein ebenso zusammengesetzter schwimmhautartiger Saum verbindet die Basen der Lappen.

Die Rhopalien konnten nicht näher untersucht werden, scheinen sich durch Kleinheit und Einfachheit der Sinnesgrube und Fehlen des Pigments denen der einfacheren triptera zu nähern.

Bei der bisher unsicheren Stellung von Crossostoma erscheint es geraten, wie bei einer neuen Gattung, die verwandten Gattungen mit ihren verbindenden und trennenden Merkmalen zum Vergleich heranzuziehen. Es kommen zunächst die dreiflügligen Formen mit kolbigen Armanhängen in Betracht. Die Gattung Mastigias besitzt Gallertkolben und ist auch ähnlich in Bezug auf den Zerfall der Flügel in einzelne Läppchen; doch sind die Kolben anderer Art, wie bei Mastigias erörtert ist: ein sehr starker Endkolben, fur den hier das Homologon fehlt, und zahlreiche kleine Kölbchen, die höchstens mit den hier vorkommenden, zwischen den Krausen sitzenden, nicht mit den grösseren, verglichen werden können und bei Mastigias meist uibersehen wurden (vgl. p. 62). Ferner ist bei Mastigias der Zerfall in Läppchen vorwiegend an den Dorsalflügeln zu erkennen; ausserdem fehlen die Peitschenfilamente, ganz abgesehen von den beträchtlichen Unterschieden im Canalsystem und dem Gastrogenitalkreuz. Für die Gattung Eucrambessa, die VANHöFfEN mit Mastigias vereinigen will, gilt auch, wenn sie bestehen bleibt, dasselbe bezuiglich der Unterschiede von Crossostoma wie für Mastigias. Die in nächste 
Nähe gehörige neue VANHöffev'sche Gattung Desmostoma besitzt zwar Peitschenfilamente, jedoch nur in der Centralscheibe, nicht auf den Armen; bezüglich der Kolben gilt das Gleiche wie von Mastigias; auch wird fiir die Armflugel kein Zerfall in Lappen beschrieben oder abgebildet, der, wenn vorhanden, einem so sorgfältigen Darsteller wie VANHöFFEN gewiss nicht entgangen wäre. Es könnte dies durch die Jugendlichkeit der Exemplare bedingt sein, doch zeigt schon das GoETte’sche kaum grössere, den Zerfall sehr deutlich.

Immerhin ist diese Gattung noch die nächstehende und beide könnten, wenn man die Diagnosen nicht etwas schärfer fasst, wie VANHöfFEN, zusammenfallen. Ob dann der AgAssiz'sche oder VAnhöffen'sche Name für das Genus erhalten bleiben solle, hängt von der Auslegung der betreffenden Regeln ab. Einstweilen halte ich die Formen nicht nur für spezifisch, sondern auch für generisch verschieden.

Von dreifligligen Formen kommen noch ferner Lychnorhiza und Phyllorhiza in Betracht, in deren nächste Nähe VAxhöffex Versura und somit auch Crossostoma gestellt hat, wegen der "unzweifelhaft dreiflugligen Arme, den sehr breiten Subgenitalostien und der Ringmuskulatur" (I888, p. 42). Die Aehnlichkeit mit Lychnorhiza wird noch erhöht durch die von HaÉcKel dort beschriebene Lappenbildung der Fligel und die Peitschenfilamente. Dagegen hat Lychnor/iza keine Kolben und ein ganz anderes und sehr charakteristisches Canalsystem ( 1879, Taf. 34, Fig. 2). Das Fehlen der Kolben unterscheidet auch Phyllorhisa, ebenso wie die "Kleeblattform der Arme"; doch zeigt eine von Lendenfeld als Phyllorhiza punctata beschriebene Form (I888), mit der obenbeschriebenen Crossostoma einige Aehnlichkeit, und ich muss deshalb etwas auf diese Gattung eingehen, umsomehr dieselbe nur ein sehr problematisches Litteraturdasein seit L. AGAssiz fristet, und die betr. Lendenfeld'sche Art von VAniröffen nicht in sein System aufgenommen wurde, weil es ihm wie bei Lendenfeld's und HaAcke's Pseudorhiza "nicht möglich war, nach den vorhandenen Beschreibungen und Abbildungen ein sicheres Urteil über die Gestalt ihrer IIundarme zu gewinnen". In Anbetracht der viele Seiten langen Beschreibung und der zahlreichen Figuren LENDENFELD'S ist dies Urteil streng, aber nicht ungerechtfertigt; doch ist mildernd $2 u$ bedenken, dass die Gattung Phyllorhiza, auf die Lendenfeld seine Art zu beziehen suchte, von L. Agassiz nach Skizze und Notiz eines Andern, ohne Abbildung mit einer sehr kurzen Diagnose aufgestellt war und bei HAEClEL ebenso fragmentarisch wegkommt, trotzdem er sie um eine „neue, sehr ähnliche Art” nach einem schlecht erhaltenen, verstümmelten Spiritusexemplar vermehrt hat. Die Lexdexfeld'sche Beschreibung der Arme „mit Schulterplatte und Terminalplatte, mit ihrer membranösen Gestalt und ihren Falten" (I888, p. 225) möchte ich auf drei Flügel mit platten Seitenästen wie hier und bei Lychnor/iza etc. deuten. Kolben fehlen auch der Lendexfeld'schen Form; dagegen sind die unteren Filamente sehr stark; die oberen nehmen an der Mundscheibe Teil an einer rosettenartigen Bildung (I888, p. 226); auch ist das Canalsystem, die Muskulatur und die Randlappen den oben und den von Versura beschriebenen Verhältnissen nicht unähnlich. Einen sicheren Anschluss ebensowenig wie eine sichere Trennung möchte ich mir aber auf Grund der LeNDENFELD'schen Beschreibung nicht erlauben; es ist mir mehr um den Hinweis zu thun, wie problematisch die ganze, sonst, so viel mir bekannt, nirgends wieder beschriebene Gattung Phyllor/iiza ist. Die eigentiumliche und mit allen anderen Verhältnissen schwer zu vereinbarende "Kleeblatt"-Form des Arms, die die Diagnose bildet, 
diurfte wohl durch solche plattigen, weiter gelappten Unterarmteile, wie hier vorgetäuscht sein, zumal wenn sie im Wasser flottieren, und keine generisch eigene Bildung darstellen. Auch die vermittelnde Stellung zwischen Cotylorhiza und Multicrispaten, die man nach CLaus dem Genus Phyllortiza zuschreibt, stimmt mit dem oben von der Armform bei Crossostoma Gesagten überein.

LENDENEELD hat noch bei seiner Art angegeben, dass man nach dem HAECKEL'schen Schliussel seine Phyllorhiza nicht unterbringen könne (I888, p. 230) oder zu Stylorhiza komme "nderen Arme aber anders gebaut sind", und die laut HAEckel bei Cotylorhiza und bei den Versuriden steht. Auch dies scheint mir einen Hinweis auf meine obige Deutung der LEvDExFELD'schen Art zu geben, wie auf die erörterten Verwandtschaftsverhältnisse ïberhaupt.

Die Gattung Stylorhiza selbst ist übrigens wie oben bei den dichotoma bereits bemerkt, eine sehr problematische und passt durch die breiten Subgenitalostien und u. a. durchaus nicht zu den übrigen Vertretern dieser Gruppe. Da auch sie nur nach einer alten Abbildung aufoestellt und die Stammart nur durch eine „sehr ähnliche Art” von HaEckel „nach einem mässig convervierten und teilweise verletzten Spiritusexemplar" vermehrt wurde, so dürfte sie ein ähnliches Schicksal erfahren wie Phyllorhiza und mit dieser zusammenfallen, resp. zusammen "fallen".

Die bisher angeführten Unterschiede und Aehnlichkeiten anderer Gattungen gelten für Crossostoma wie für Versura zusammen, abgesehen von dem iber die Peitschenfilamente Gesagten, da ja solche bei Versura fehlen. Dazu käme noch, allen erwähnten Gattungen, wie auch Versura gegenüber, die betreffende Zottenrosette, die der Gattung Crossostoma auch den Namen verliehen hat. Die Peitschenfilamente können hier kaum als Sexualcharakter aufgefasst werden, da sie ja nicht nur an der Armscheibe, sondern auch noch weit unten am Arm selbst vorkommen; die Mundrosette im berichtigtem Sinn (gegen Tilesius etc.) darf wohl mit HaÉCKEL als ein nicht blos spezifisch, sondern generisch trennendes Merkmal gelten. Es kommen aber auch noch andere Unterschiede zwischen Crossostoma und Versura dazu, so bei ersterer die extreme Streckung des Gastrogenitalkreuzes und die damit zusammenhängende Abweichung im interradialen Canalsystem, ferner die Teilung der Muskulatur bei letzterer und die verschiedene Exumbrellarstructur. So kennzeichnet sich die Gattung Vorsura als mit dreiflügligen, secundär tiefgelappten Mundarmen, mit zahlreichen, gleichartigen Kolbenanhängen, ohne Peitschenfilamente und ohne centrale Rosette, mit besonderer circulärer Muskulaturanordnung.

Es eribrigen beim Vergleich von den tripteren noch diejenigen Formen, die sich durch den Nangel jeglicher Armanhänge leicht hiervon unterscheiden. Unter diesen Gattungen kommt Loborhiza Vanh. durch die secundäre Lappenbildung der Armfluigel und durch das anastomosirende Canalnetz noch der, Versura-Gruppe am nächsten zu stehen. Die Gattung Toxoclytus mit einfachen Armen ohne Anhänge, die von VANHöffex aufgelöst wurde, ist von L. S. Schultze wieder aufgestellt worden. Deren Unterschiede von der noch uibrig bleibenden wichtigen Gattung Crambessa, zu der VanhöfFen die eine Toxoclytus-Art gestellt hat, beruhen soviel ich L. S. Schultze's Beschreibung entnehme eigentlich nur in der Trennung der Subgenitalhöhlen gegenüber dem einheitlichen Porticus von Crambessa. Das Canalsystem, wonach laut L. S. Schultze selbst, eine zukünftige Systematik sich mehr zu richten haben wird, ist bei beiden sehr ähnlich (s. o. p. 54). 
Mastigias L. Ag. 1860. - Hacckel 1879.

Sensu emend. Vanhöffen is88.

Sensu restrictu Maas.

Rhizostomee mit dreiflügligen und meist noch seitlich gefiederten Mundarmen, bei denen der Saugkrausenbesatz nicht bloss a uf die 3 Kanten beschränkt bleibt, sondern a uch auf die seitlichen Flächen übergreift. Der Unterarm selbst endigt in einen ansehnlichen (dreikantigen bis drehrunden) Gallertkolben. Zwischen den Armkrausen finden sich zahlreiche, kleinere gestielte Kolbenanhänge. Canalsystem mit zahlreichen (6-io) anastomisierenden Radiärcanälen zwischen den $S$ Rhopalarcänalen und mit schwachem Ringcanal und extracirculärem Netz. Muskulatur ringförmig mit 8 radialen Unterbrechungen.

Die Definition und Abgrenzung des Genus Mastigias ist bei den bisherigen Autoren eine sehr verschiedene. Die Endung ias bezeichnet etwas unschönes, tadelnswertes; der Name bedeutet also nicht, wie HAECKEL sonderbarerweise übersetzt: „fauler, peitschenswerter Knecht”, sondern "unförmliche Geissel”. Das Hauptkennzeichen der Gattung ist meiner Ansicht nach in der That der grosse Gallert- oder Geisselkolben, der morphologisch wohl den distalen, krausenlosen Teil des Unterarms darstellt. Laut HAECKEL's ausdrücklicher Bemerkung (I 879, p. 622) sind die "Saugkrausen ohne besondere Anhänge", und er hat aus einer Form, die noch ausserdem zwischen den Armkrausen Gallertkolben trägt, eine besondere Gattung Eucrambessa gemacht. VANHöfFEN macht darauf aufmerksam, dass auch Mastigiasformen solche kleinen Kolben tragen können (so z.B., laut ausdrücklicher Beschreibung von L. Agassiz: Hidroticus rufus aus der HaEckeL selbst seine $M$. occellata gemacht hat), und zieht deshalb die Gattung Eucrambessa ein. Auch giebt er neuerdings eine noch mehr erweiterte Diagnose der Gattung, weil er unter dem ValdiviaMaterial eine typische Mastigias papua ohne Endkolben neben einem kleineren Exemplare mit langen Endkolben fand. Seine neue Diagnose lautet: „Rhizostome Meduse ohne Schulterkrausen, mit dreikantig pyramidalen Mundarmen, die Gallertknöpfe tragen wobei gleichgültig ist, ob diese als Endknöpfe der Arme oder zwischen den Armen entwikelt sind" (Ig02, p. 47). Ich kann ihm darin nicht folgen, sondern halte den gallertigen Endkolben für eine morphologisch andere Bildung wie die kleinen, gesetzlos zerstreuten Kolbenblasen zwischen den Armkrausen. Dass der Kolben vielleicht einmal abnormerweise fehlen kann, bestreite ich nicht, um so weniger als ich ihn gerade bei jüngeren Stücken in wechselnder Länge ausgebildet fand. Wenn man aber, wie VANıöfFex soweit geht, das Fehlen und die Stellung der Kolben zu den Variationsmöglichheiten zu rechnen, darf man gewiss nicht, wie er es gethan hat, die Länge des Kolbens unter die Art Charactere aufnehmen (1. c. p. 49). Der Kolben ivächst als Unterarmende hervor, oft verschieden schnell, wie ich an vielen Jugendexemplaren sehen konnte (s. u. p. jo), und könnte daher auch noch nachwachsen. Auch die "dreikantig pyramidale" Form in Vanhörfen's Diagnose ist für die Mundarme nicht ganz kennzeichnend; der Unterschied von dreifüglig und dreikantig ist nicht durchgreifend (s. o.); gerade für Mastigias ist ein Zerfall der Flügel in einzelne Läppchen charakteristisch, und ein Uebergreifen der Krausen auf die Flächen wird 
durch solche seitliche Läppchen bewirkt. Dass ich auch die Verzweigung des Canalsystems und die Muskulaturverteilung unter die Gattungsmerkmale aufnehme, rechtfertigt sich durch den Vergleich zahlreicher dreifluigliger Formen und der Rhizostomen uiberhaupt. Ueber die Gattung Eucrambessa ist einstweilen nichts Sicheres zu sagen so lange nicht ersichtlich ist, ob mit den kleineren Kolben bei letzterer nicht doch etwas anderes als die auch bei Mastigias vorkommenden Kolbenblasen gemeint ist.

Die Nichtbeachtung der kleinen Kolben bei Mastigias seitens HAECKEL hat noch weitere Verwirrungen im Gefolge gehabt. Kishinouve beschreibt ( $1895 \beta$ ) eine neue Mastigias physophora, und L. S. Schultze hält dieselbe für wahrscheinlich mit $M$. papua identisch, da sie nur Abweichungen in den Armmaassen zeige, welche ja variabel seien. Schultze beklagt mit Recht (1898, p. 445), „dass Kishinouye weder eine Diagnose der neuen Art giebt, noch bei der Beschreibung auf die ubrigen Mastigias-arten Bezug nimmt". Soviel ich aus der Beschreibung des japanischen Forschers entnehme, hält er das Vorkommen von kleinen Kolbenblasen bei seiner Art für etwas Neues und hat ihr auch danach den Namen "physophora" gegeben; denn er musste nach HAECrEL's Monographie, wenn er nicht andere Beschreibungen dazunahm, annehmen, dass dies bei keiner Mastigias vorkäme. Bei M. orsini beschreibt aber VAnHöffex solche Blasen, Chun erwähnt bei seiner neuen $M$. siderea "die kurzen gestielten Nesselkolben" an den Saugkrausen; ich habe die Kolben an dem mir vorliegenden Material an allen Exemplaren, ohne Rücksicht auf Unterschiede in Geschlecht, Färbung, Armmaassen, stets gefunden. Sie gehören daher ebenso wie der grosse kolbenförmige Endteil des Arms zur Charakteristik der Gattung.

Die bekannten Mastigias-Arten werden von VANHöFFEN unter Einrechnung von Eucrambessa (M.) Mïlleri zusammengestellt und nach Zahl der Velarlappen, nach dem Verhältnis von Arm, Kolben, und Schirmradius, sowie endlich nach Zeichnung unterschieden. Früher wurde noch die Gestalt der Lappen hinzugefügt. Es ergeben sich:

M. papua Lesson, (L. Ag.). $8 \times 8$ halbkreisförmige Velarlappen. Arme $=$ r. Kolben $=2 \mathrm{r}$ (wern vorhanden). Schirm bläulich (grün) mit grossen weissen Flecken.

MI. occellata Modeer. $8 \times 6$ oder (Haeckel) $8 \times$ I 2 stumpfabgerundete Velarlappen. Arme kürzer als $r$. Kolben $=$ r. Rotbraune Exumbrella mit helleren, braungerandeten Flecken.

M. pantherina Haeckel. $8 \times$ i 6 schmale, rechteckig abgestutzte Velarlappen. Arme fast 21 . Kolben $=4-6 r$. Exumbrella braun mit weissen, schwarzgeränderten Flecken.

11. siderea Chun. $8 \times 8$ abgerundete Velarlappen. Arme $=2 \mathrm{r}$. Kolben $=\mathrm{r}$. Exumbrella gelb mit weissen Flecken. Ausserdem Tüpfelung, auch auf der Subumbrella.

M. orsini Vanhöffen. $8 \times 16$ spitze, schmale Velarlappen. Arme $=r$. Gallertknopf $={ }^{1} /{ }_{4} r$. mit breiter Basis aufsitzend. Farbe?

11. physophora Kishinouye. $8 \times 8$ runde Velarlappen. Arme $=r$. Kolben $=$ r. Schirm hellbraun mit dunklen Flecken.

Mir scheinen nicht alle Arten als solche haltbar zu sein, sondern eventuell nur locale Varietäten darzustellen, vielleicht auch ganz zusammenzufallen. Eine Betrachtung der einzelnen Merkmale auf Grund des von der Siboga vorhandenen reichen Mastigias-materials wird das wohl erweisen. 
Die Zahl der Randlappen wird immer wieder als Merkmal angefuhrt, trotzdem die einzelnen Autoren (ausser HAECKEL) darin übereinstimmen, dass deren Verhältniss im Lauf der Entwicklung sich sehr ändert. CHUN erwähnt (1896, p. I 4), dass Jugendformen von siderea 4 Velarlappen im Antimer zeigen, ältere 5 oder 6, die sich bereits zu teilen beginnen; giebt aber doch für die Art So Randlappen, also 8 pro Antimer, ausser dem Rhopalar-Doppellappen, an. In zutreffender Weise hat L. S. Schultze darauf aufmerksam gemacht, dass ganz verschiedene Dinge von verschiedenen Autoren als Randlappen in Rechnung gebracht werden (1898, P. 445); , ist es immer der durch eine tiefere Exumbrellarfurche von seinem Nachbar getrennte, oder jeder aus der Peripherie vorspringende Abschnitt des Schirmrands?" Von den tieferen eingeschnittenen können einige distal gespalten sein, andere nicht; eventuell werden sich die einfach endenden auch noch spalten, oder wie ich nach meinen Beobachtungen annehmen muss, kann eine distale Spaltung zuerst auftreten und sich dann auf die Exumbrella als Furche fortsetzen. Nan hat also zweierlei Arten von Randlappen, periphere und exumbrellare vor sich, und je nach Zählmodus und Altersstadium können sich recht verschiedene Gesammtzahlen ergeben.

Von der hier vorliegenden Art, die als eine besondere Varietät zu papua zu stellen sein wird, sind alle Altersstadien von kleinen $7 \mathrm{~mm}$. im Durchmesser haltenden Exemplaren mit noch offenen Mund bis zu solchen von I $20 \mathrm{~mm}$. Durchmesser mit reifen Gonaden vorhanden. Die juingsten zeigen zwei Lappen zwischen je 2 Rhopalien, von denen der eine sich bereits zu teilen beginnt. (Diesem Stadium vorangehend ist das auf die Ephyra folgende mit nur einem Zwischenlappen, das CuUn abbildet (I896) zu deuten). An Exemplaren von I $2 \mathrm{~mm}$. sind bereits beide Lappen zweigeteilt und die Einschnitte aller 4 reichen fast gleich hoch hinauf, wenn auch die frïhere Zweiteilung durch Grössen Unterschiede noch zu erkennen ist (Fig. 54). Man kann also von 4 Hauptlappen reden. Deren weitere Teilung in Nebenlappen geht etwas unregelmässiger, langsamer vor sich, sodass die Vierteiligkeit noch auf Stadien von $40 \mathrm{~mm}$. Durchmesser erkennbar ist. Mitunter teilen sich alle 4 Lappen gleichzeitig von der Peripherie her (Fig. 55), mitunter gehen zwei darin voran und zeigen dann oft schon eine tiefe Exumbrellarfurche, während die anderes noch ungeteilt sind oder erst Spuren von peripheren Kerben erkennen lassen. Welche von den 4 Lappen es sind, die in der Teilung rorausgehen, ist nicht bestimmt, mitunter die beiden äusseren; dann entsteht ein symmetrisches Bild (Fig. 56); mitunter erscheinen aber auch weniger reguläre, ebenfalls 6 teilige (wie Fig. 57). Beide sind nur Vorbereitung fur ein $S$ teiliges Stadium, das nunmehr zu persistieren und höchstens durch unregelmässige Einschübe (9 teiliges Antimer) oder durch unvollkommene Zweiteilung einzelner Lappen etwas iberschritten wird. Nit dieser Lappenzahl kann man sich bei sämmtlichen Arten $(\delta \times(\delta+2)=80$ Lappen) abfinden, ausser bei ocellata, pantherina und Orsini. Bei occllata werden I 2 Lappen von HAEckEL, also I 2 pro Antimer + 2 Rhopalarlappen als charakteristisch aufgefuhtrt; es ist aber klar, dass diese Zahl dann erreicht wird, wenn von 8 Lappen sich 4 geteilt haben, 4 noch nicht, also in einem vorbereitenden Stadium zum r 6 lappigen wie es für panthcrina $[8 \times(16+2)=144$ Lappen] charakteristisch sein soll. Auch Vanhöffen beschreibt als ocellata eine Form, mit nur 6 Randlappen pro Antimer, von denen sich einige bereits teilen, eine Combination, die aber auch bei Arten mit nur so Lappen als jügeres Stadium rorkommt. Es ist also fraglich, ob iberhaupt die Lappenzahl verwandt werden darf; immerhin 
scheinen die Formen mit so sehr erhöhter Lappenzahl verschieden, aber unter ihnen besteht auch nach anderen Merkmalen, wie zwischen pantherina und occollata, nur ein gradueller Unterschied.

Die Form der Randlappen wird bei den Arten ebenfalls verschieden angegeben, einmal als kreisrund, einmal als rechteckig und abgestumpft, einmal als schmal und spitz. Doch hängt dies ebenfalls zum guten Teil von Wachstumsunterschieden ab. Im Jugendstadium, wenn nur wenige Lappen zwischen 2 Rhopalien vorhanden sind, sind sie halbkreisförmig (Fig. 54), später werden sie mehr und mehr rechteckig und stumpf abgerundet (Fig. 57) und entsprechend schmäler. Ganz schmale und spitze Lappen kommen nur bei den oben erwähnten Formen mit sehr hoher Lappenzahl vor. Die Rhopalarlappen sind überall übereinstimmend schmäler und herzförmig.

Die Längen-Verhältnisse von Ober-und Unterarm und Kolbenanhang zum Schirmdurchmesser wechseln etwas in der Ontogenese, je nach dem die Endanhänge mehr oder minder schnell vorwachsen; nachdem aber einmal die ersten Unregelmässigkeiten überwunden sind (s. auch u. p. 70 ), bleiben sie an meinem Material von einem gewissen Stadium an ziemlich constant, etwa von der 4-Teiligkeit des Lappenrandes an gerechnet. Nach einer Reihe von Maassen, die ich an Exemplaren von $25 \mathrm{~mm}$. bis etwa I $20 \mathrm{~mm}$. ausgefuhrt habe, ist das durchschnittliche Verhältnis Oberarm : Unterarm: Kolbenanhang: Schirmdurchmesser $=2: 5:$ IO : $\mathrm{x}_{4}$. Am meisten schwankt dabei noch der Kolbenanhang, dem man öfters 12 und mehr als Verhältniszahl zusprechen kann, aber nie mehr als dem Durchmesser. Diese Zahlen stimmen fast genau überein mit den aus Beschreibung und Abbildungen ersichtlichen für die meisten Arten, ebenfalls wieder mit Ausnahme derer mit auffällig erhöhter Lappenzahl. Bei pantherina wird ein extrem langer Endkolben angegeben, occellata mit kürzerem, könnte auch hierin ein vorbereitendes Stadium dazu darstellen; orsini hat uberhaupt andersartige ganz kurze und vierkantige Endkolben.

Die Färbung, die noch verbleibt, ist an und für sich für die Artabgrenzung ein sehr problematisches Merkmal, so gut sie mitunter bei Medusen für Localvarietäten zu benutzen ist. Doch wechseln auch die Arten am gleichen Ort vielfach in Intensität und Nuancen. Manchmal trägt auch der Gehalt an symbiotischen Algen wesentlich zum Farbenton bei. Die für pantherina und occellata untereinander angegebenen Unterschiede sind nur in der Tonstärke begründet; bei beiden ist eine helle Augenfleckung auf dunkler Exumbrella vorhanden, und je nach dem dieser Ton tiefer braun oder heller rot ist, erscheint auch die Umrandung der Flecke schwarz oder nur braun. Von den andern Arten ist die Zeichnung immerhin merklicher verschieden. Von orsini liegen keine Farbenangaben vor. Eine radiäre Zeichnung wird nur bei einigen Arten (papua selbst, sidcrea, und den eventuell hierhergehörigen Pseudor/hiza-formen) erwähnt; bei den letztgenannten ist auch der Endanhang gefärbt. In beidem ist die Sibogavarietät hier anzuschliessen; die Unterschiede, auch in der Färbung einzelner Organsysteme, sind recht relativ; auf den Hauptton sind die anderen Nuancen entsprechend gestimmt. Eine besondere Tüpfelung, die auch auf die Subumbrella übergreift, wird von Cirus für siderca als charakteristisch erwähnt, aber auch für papua beschreibt neuerdings VANIÖFrEN ähnliches (1902, p. 47). Hier ist ebenfalls eine sehr ausgesprochene Tüpfelung vorhanden; dieselbe rührt aber hier, ohne dass ich dasselbe von siderea behaupten wollte, von nesterweise verteilten gelben 
Zellen her, die bei keinem Exemplar fehlen. Bei siderea wird laut CHun die gesammte Braunfirbung von solchen Zellen hervorgerufen. Nach alle dem dürten sich folgende Arten als sicher herausstellen:

31. pafua und ausser der als solcher unterscheidbaren typischen Form:

var. Sibogae mihi, var. siderea Chun, var. physophora Kishin.

M. occellata Modeer (Haeckel); hierunter fällt M. pantherina.

Es verbliebe noch $M$. orsini Vanhöffen, die in jeder Beziehung gut charakterisiert ist, die aber ein so verschiedenartiges Canalsystem hat, dass sie meiner Ansicht nach aus der Gattung Mastigias iiberhaupt auszuscheiden ist und bei Crambessa, oder als besondere Gattung aufzustellen ist. Auch die Kolbenverhältnisse nehmen eine Mittelstellung zwischen Mastigias und manchen Crambessa-arten ein.

Für die geographische Verbreitung ist bemerkenswert, dass sämmtliche Arten und Varietïten dem indopazifischen Gebiet angehören und hierin eine grössere oder nur beschränktere Verbreitung zu besitzen scheinen. Orsini ist nur aus dem roten Meer bekannt, papua in der typischen Form, wie in den Varietäten am weitesten verbreitet.

I. Mastigias papua Lesson, L. Ag. nov. var. Sibogac.

Taf. VI, Fig. 54-57. Taf. VII, Fig. 58, 59, 6I, 64. Taf. VIII, Fig. 75, 76, 77. Taf. IX, Fig. 84, 85. Taf. XII, Fig. I IO.

\footnotetext{
Makassar. I Exemplar von etwa $4 \mathrm{~cm}$. Durchmesser.

Stat. I25. I erwachsenes Exemplar etwa $12 \mathrm{~cm}$. Durchmesser.

Stat. 299. 4 Exemplare von etwa 3, 4, 5 und $6 \mathrm{~cm}$. Durchmesser.

Stat. 3 I3. I erwachsenes Exemplar von fast i $2 \mathrm{~cm}$. Durchmesser.

Saleyer. 4 Exemplare von ungefähr $2^{1 / 2}-5 \mathrm{~cm}$. Durchmesser.

Saleyer. 6 Jugendexemplare von $0,7-2^{1 / 2} \mathrm{~cm}$. Durchmesser.
}

Schirm mäsig gewölbt, Velarlappen rechteckig mit abgerundeten Ecken. Ostien des Subgenitalporticus dreimal so breit als die Pfeiler; $z w i s c h e n$ je 2 Rhopalargefässen $7(-I 0)$ Radiärgefässe von zuerst gestrecktem Verlauf. An den Armen zahlreichekleine runde Kolbenblasen; Endanhang fast so lang als der Schirmdurchmesser, deutlich dreikantig.

Grundfärbung gelblich? mit orange? Tiipfelung; keine Augenflecke auf der Exumbrella. Subumbrella mit $S$ violetten Radiärbändern auf den Rhopalarcanälen. Anhangskolben violett gesprenkelt. Canalsystem rosa; Gonaden orange.

Dic Mundarme der Mastigias-arten sind fur die Ableitung der verschiedenen Armtypen unter den Rhizostomen von Bedeutung, und zeigen je nach den Altersstadien sowohl Beziehungen zu den einfachen dreiflugeligen Formen (tripteren VAniöffen's) wie Ljchnorhiza, als auch zu den divergentesten mit Schulterkrausen (scapulaten) wie Rhopilema. Es sind diese Verhältnisse bisher wenig gewürdigt und sollen darum auf Grund des hier vorliegenden Jugendmaterials Darstellung finden. Die jüngeren Stadien zeigen deutlich, dass der Krausenbesatz nur auf 
die Kanten der drei Flügel beschränkt bleibt; da wo der Gallertkolben abgeht ist sogar eventuell eine direkte Unterbrechung des Krausenbesatzes zu sehen, so dass die 2 abaxialen und die axiale Krausenreihe gar nicht mit einander zusammenhängen. Die Form des Arms ist namentlich im oberen Teil deutlich flügelig, nicht pyramidenförmig dreikantig; man kann die Flügel von der abaxialen Seite auseinander klappen (Fig. 76) und in die tiefe Rinne zwischen beiden hineinsehen. In normalem Zustand liegen die Fliugel aber dicht aneinander so wie es aus der Profilansicht (Fig. 75) und aus der Ansicht von der axialen Kante (Fig. 77) hervorgeht. Nach dem Endkolben zu wird die Rinne zwischen den Fligeln immer seichter, und die dreiflugelige Form geht so in die dreikantige ïber; der Kolben selbst zeigt, je nach dem Erhaltungszustand mehr oder minder deutlich, dreikantige Form (Fig. 75 und 77). Diese Weiterbildung von dreiflugeligem zu dreikantigem Armzustand schreitet auch in der Ontogenie fort. Hand in Hand damit geht auch das Uebergreifen der Krausen auf die Flächen, sodass sich die Krausenreihen nicht erst an der Spitze der Pyramide treffen. Fernerhin sondern sich dann mehr und mehr einzelne Krausenästchen ab, wie es schon HAEcres vermerkt (I879, p. 623) und L. S. Schultze ( 1897 , p. I62) nach welchem, die drei Flügel der Arme in eine Anzahl selbstständiger, nur an der Basis zusammenhängender Krausenbündel zerfallen". Diese Basis wird hier von dem langen dreikantigen Stamm des Unterarms gebildet, der in den Endkolben ohne deutliche Grenze übergeht. An dieser Spaltung scheinen mir aber bei der vorlïgenden Art nicht alle Teile des Arms gleichmässig teilzunehmen, sondern vorzugsweise die oberen. Hier trennen sich zwei besonders starke Aestchen, je einer fur jede abaxiale Krausenkante, ab (Fig. 84 und $85 z$ !! ) und gewinnen eine gewisse Selbstständigkeit gegenüber dem gesammten unteren Teil, an dem die einzelnen Aestchen viel kleiner und weniger gesondert bleiben. Man kann geradezu zu sagen, dass sich zwei Schulterflügel von einem distalen trigonen Teil abzutrennen beginnen. Dies Verhältnis ist wichtig, weil es zeigt, wie man sich die Ableitung der merkwürdigen Schulterkrausen der scapulata denken kann; denn es ist nur ein gradueller Unterschied von diesem Stadium bis zu dem der jungen Pilema-formen (Fig. 82). Ich glaube daher auch nicht wie CLAUS, dass die Schulterkrausen eine Neubildung darstellen, weil sich ihre Krausenreihen in der Entwicklung ganz unabhängig von denen der Arme gleich in loco bilden ( $188_{3}$, p. 49), sondern leite sie auf Grund der vergleichenden Anatomie von abgesprengten Unterarmkrausen-teilen, wie HAECKEL, nur in etwas anderer Weise, ab.

Nicht ohne Bedeutung für die Auffassung der Arme ist, wie auch DéLAge und Hérouard hervorheben (Coelenterés, p. 356), die Verzweigung ihrer entodermalen Canäle. Man kann nicht sagen, dass der Haupstamm des Arms den Krausenreihen entsprechend in 3 Aeste zerfiele, auch nicht, dass sich ein Längstamm finde, der nach Abgabe der Aeste für die abaxialen Krausen zurück bleibe und sich als solcher in den Gallertkolben fortsetze, sondern an einer Stelle, dem kritischen Y löst sich das ganze Canalsystem auf. Wenn die beiden Aeste fü die rechte und linke abaxiale Krausenkante abgegangen sind, so löst sich gleichzeitig der Rest in zahlreiche ventrale Aestchen auf (Fig. 84 und 85 ent). Diese gehen anstatt, wie vor der Teilung rechtwinklig, in immer spitzerem Winkel von dieser Teilungsstelle ab; der Canal des Endkolbens ist das letzte dieser Aestchen, aber ebenfalls hoch hinauf bis zur $\mathrm{Y}$ Teilungsstelle zu verfolgen. Die Canäle für die beiden Krausenkanten gabeln sich jeder in den Ast für den selbstständigen 
Dorsalfligel und in einen Restast, der sich dann in die Aestchen für die einzelnen Krausen des trigonen Teils auflöst (Fig. 84 ent).

Der Centralmagen zeigt eine ausgesprochene Kreuzform, die perradialen Kreuzschenkel sind jedoch nicht so extrem lang, wie bei den loriferen (s. HAECkEL Taf. XXXVIII, Fig. I), sondern ungefähr von der Grösse des quadratischen Centralteils, in dem sie zusammen kommen (Fig. 58). Die 4 perradialen Gefässe sind infolge der Kreuzform des Magens viel kürzer als die 4 interradialen, alle 8 aber sind sie durch intensivere, fast dunkelviolette Färbung vor den iibrigen mehr rosa-violetten Teilen des Canalsystems ausgezeichnet; dadurch entstehen 8 violette, zu den Rhopalien ziehende Radiärbänder auf der Subumbrella, die am längsten sichtbar bleiben, wenn durch die Formoleinwirkung die iibrige Färbung bereits ausgezogen ist. Zwischen je 2 Rhopalarcanälen entspringen schon bei jungen Exemplaren (von 7 mm. Durchmesser) typisch 7 Radiärcanäle; diese unterscheiden sich im Jugendstadium nur sehr wenig im Caliber von den Rhopalarcanälen, auch sind sie wie diese kaum verästelt, sondern verlaufen in auffallend gestreckter Richtung (Fig. 59), bis sie durch Anastomosen einen Ringcanal bilden, jenseits dessen das Netz etwas dichter wird. Später wird eine Anastomosen- und Blindsack-bildung auch mehr central wahrgenommen (Fig. 6I); immer noch zeigen aber die einzelnen Gefässe einen gerade gestreckten Lauf in der ersten Strecke. Das Stadium mit 7 Zwischencanälen dauert sehr lange an und entspricht so dem Zustand mit 4 je zweigeteilten Zwischenlappen am Schirmrand; noch Exemplare von mehreren Centimeter Durchmesser besitzen nicht mehr Zwischencanäle wie solche von mehreren Nillimeter, nur sind die Canäle etwas mehr verästelt, ohne jedoch im ganzen Netz die deutliche Radiärtendenz des Verlaufs aufzugeben. In späten Stadien crst vermehren sich die Radiärcanäle noch etwas an Zahl, lange jedoch nicht im Verhältnis zum Wachstum; es sind anstatt 7 alsdann 8 oder 9 , selten 10 zwischen je 2 Rhopalarcanälen $z$ u erkennen; die Gabelstellen, liegen dann viel näher der Austrittsstelle als im Jugrendstadium; immer aber zeigt das Anastomosennetz noch Radiärtendenz (Fig. 58). In den Ringcanal treten ungefähr doppelt so viel Canäle ein, als aus dem Magen hervorgegangen sind (also etwa 20 pro Antimer), und die wiederum verdoppelte Zahl tritt aus ihm aus. Derselbe stellt also eigentlich nur eine Zone des Anastomennetzes dar, die eine plötzliche starke Verïstelung vermittelt. (Auf der Fig. 58 ist er zu breit geraten). Im peripheren Teil ist eine Radiärtendenz der Canäle nicht mehr wahrnehmbar. In die Lappen gehen Teile des Netzwerks hinein mit entsprechend tieferen und seichteren Einschnitten für Haupt- und Nebenlappen. Die 8 rhopalaren Canäle haben sich auch noch in den vorgericktesten Stadien von der Gabelung und dadurch von dem Aufgehen ins Netzwerk frei gehalten und treten deshalb, auch abgesehen von ihrer Färbung, durch Caliber und gestreckten Verlauf deutlich hervor (can rh).

Die Exumbrella ist gleichmässig gewölbt, bei jüngeren Tieren etwas stärker als bei erwachsenen. Sie zeigt ausser der erwähnten Tüpfelung keine besondere Zeichnung in Form von Augenflecken (s. 11.).

Die Armscheibe hat eine sehr charakteristische Gestalt; ihre perradiale Begrenzung fällt mit der Begrenzung der Magenlinie zusammen und bildet den gleichen ausgeschweiften Bogen (Fig. 58); interradial bildet sie dagegen eine fast gerade Linie. Die ganze Armscheibe erhält dadurch fast eine quadratische Gestalt, wie sie laut HAECKEL (1879, p. 615) für die 
Unterfamilie der Crambessiden charakteristisch ist. „Die vier abgestutzten Ecken des Quadrats werden von den Armpfeilern eingenommen; die vier Seiten begrenzen die Subgenitalostien". Die Oeffungen sind dadurch sehr weit; in der That ist der ganze Subgenitalsaal hier fast ein Teil der Subumbrella und von dieser nur in den Perradien durch verhältnismässig dünne Pfeilerspangen abgetrennt. Schon bei jungen Stadien ist er ein durchaus einheitlicher Hohlraum.

Die Gonaden selbst bilden 4 interradiale Hufeisen, die sich aber im Perradius nochmals zurückbiegen und einander sehr nähern, so dass im erwachsenen Zustand (von oben oder unten gesehen) fast eine einheitliche Kreuzfigur entsteht; jedoch ist die perradiale Trennung stets noch nachzuweisen. Secundäre Sexualcharaktere in dem Kolbenbesatz oder den Anhängen der Armscheibe habe ich hier nicht nachweisen können.

Die Muskulatur der Subumbrella bildet den typischen Ring, jedoch von den violetten Radialbändern breit unterbrochen, nach dem Rand zu stärker.

Die Rhopalien sind ohne erkennbare Bulbusanschwellung und darum von gestreckter Form; nur die ectodermale Differenzierung tritt ventral und seitlich hervor. Die sog. äussere Sinnesgrube ist schwach entwickelt, etwas mehr als bei Cassiopica aber weniger als bei Crambione und Crambessa, etwa so wie es Lendenfeld für Phyllorhiza angiebt (1888, Fig. 6i). Das unpaare distale Auge ist hier besonders kräftig entwickelt. An Schnitten zeigt es sich als tief eingestiilpter Becher (Fig. IO5 oc). Während die Pigmentzellen bei Crambione etc. dem Ectoderm angehören, scheint es hier als seien sie entodermaler Natur, und die Stützlamelle iber ihnen; so dass in dem dadurch gebildeten Becher besondere Ectodermzellen eingebettet liegen, also eine ähnliche Bildung, wie nach Schewiakoff (I889) das ventrale Becherauge von Aurelia. Die sonstige Analogie mit Crambione etc. macht dies nicht wahrscheinlich, und die Frage ist an meinem Material nicht sicher zu entscheiden. Die ectodermalen Nervenepithelien am Kolben selbst sind sehr gut entwickelt; die äussere Sinnesgrube zeigt sich aber hierin auch in Schnitten weniger differenziert als bei Crambione u. a.

2. Mastigias papua (Lesson), L. Ag.

Bima. 4 Exemplare von $\mathrm{I}-4 \mathrm{~cm}$. Durchmesser.

Die Exemplare zeigen deutlich die exumbrellaren Augenflecken der papua, die der var. Sibogae fehlen. Es kann dies nicht im Erhaltungszustand begründet sein (hier Alkohol, dort meist Formol), da andere Alkoholexemplare (Stat. Makassar etc.) der Exumbrellarzeichnung entbehren, und ferner ja noch weitere kleinere Unterschiede damit Hand in Hand gehen. Dieselben sind aus der obigen Diagnose von var. Sibogae ersichtlich, letztere unterscheidet sich auch entsprechend von var. siderea, mit der sie gleichen Grad beanspruchen darf. Würde var. sibogae ganz zu papua zu ziehen sein, so müsste auch siderea als Art fallen, und ist letztere eine vollkommen getrennte Art; so gilt dies auch für sibogae. Am richtigsten halte ich es darum, sie, schon weil sie untereinander und mit papua näher verwandt sind, als Varietäten einzuordnen.

ANM. Hierin werde ich auch noch durch weiteres Material von Mastigias bestärkt, das von Sansibar, dem malayischen Archipel und Japan stammt. Es sind das die von GoETTE als Versura palmata Hckl. bestimmten Rhizostomeen, die aber im Berliner Museum schon von anderer Seite (VANHÖFFEN?) eine Umbestimmung in Mastigias erfahren haben. Nachdem mir die Stucke durch die Freundlichkeit 
der Berliner Museumsverwaltung zugänglich gemacht waren, kann ich ihre Zugehörigkeit zu Mnastigias nur bestätigen und hinzufuigen, wie schwer es ist, sie ohne Kentnis der Färbung anders als allgemein auf „I. papua zu beziehen. Es sind wohl viele individuelle Unterschiede in Kolbenlänge, Lappenzahl etc. vorhanden, die sich aber durchaus auf das oben ausgeführte beziehen lassen; die Varianten erscheinen, wie schon GOETTE als für Vorsura bemerkt, am selben Ort. Auch die Tüpfelung, durch Nester gelber Zellen hervorgebracht, ist nachzuweisen, ebenso das gelegentliche Fehlen von Endkolben; kleine Blasenkolben sind dagegen stets vorhanden.

3. Mastigias spec. (Taf. VII, Fig. 60, 62, 63. Taf. XII, Fig. I I I).

Saleyer. 5 Jugendliche Exemplare von $0,8-3 \mathrm{~cm}$. Durchmesser.

Von den an gleicher Stelle gefundenen, eben beschriebenen Mastigias-formen, auch den Jugendstadien, unterscheiden sich diese Exemplare in mehrfacher Hinsicht. Zunächst fällt die geringe Grösse oder das fast gänzliche Fehlen der gallertigen Endkolben auf, noch in einem Stadium von $3 \mathrm{~cm}$. Durchmesser, während bei der typischen Form Exemplare von wenig uiber I cm. schon ebenso lange Endanhänge zeigen. Auch sind die Kolben nicht dreikantig, sondern entweder abgestumpfte Kegel (Fig. 62) oder im ausgebildeteren Fall runde Keulen mit Halsteil (Fig. 63). Man könnte dies ja zuerst für einen Entwicklungszustand der langen Kolben halten, um so mehr als VANIÖFFEN für papua beschreibt ( I 888, p. 34), „dass beim kleineren Tier nur ganz kurz gestielte Nesselknöpfe" vorhanden sind, beim grösseren diese jedoch nebst Stiel die Länge des Schirmradius erreichen". Aber hier geht dieser Zustand nicht parallel mit der Grössenentwickelung, da andere typische Exemplare schon in viel jingerem Stadium ausgebildete Kolben tragen, und ferner finden sich zusammen mit den Unterschieden in den Kolben auch solche in der Gestalt der Arme und der Ausprägung des Canalsystems. Die Arme sind hier viel gedrungener, die trigone Pyramide viel breitbasiger, und der Krausenbesatz greift in einem solchem Stadium mit "noch" kurzem Kolben schon auf die Seiten iiber (Fig. 62) im Gegensatz zum typischen Verhalten (Fig. 64). Das Canalsystem zeigt keine scharf radiär verlaufenden Zwischencanäle, sondern zwischen den Rhopalarcanälen gleich, auch bei den jüngsten Stadien, ein nahe dem Magen schon eintretende Gabelung und enge Anastomosenbildung der interrhopalaren Canäle (Fig. 60). Da die Exemplare sonst, in der Gestalt der Armscheibe, der Lappenconfiguration, dem Habitus, den typischen gleichen, so könnte man sich fragen, ob man es nicht doch nur mit individuellen Wachstumsdifferenzen zu thun habe, indem hier ein Organsystem vorzeitig entwickelt, andere Organe etwas in der Entwicklung gehemmt seien, um so mehr als ja auch im gelegentlichen Offenbleiben des centralen Mundes (s. HAEcket, p. 623) eine solche Hemmung bei Mastigias nicht selten vorkommt. Es ist ferner zu bedenken, dass VANIÖFFEN neuerdings ein typisches papza-Exemplar ohne Mundarme erwähnt.

ANn. Da mir von seiner Arbeit cinstweilen nur der Text vorliegt (dank der Freundlichheit des Verlegers), so kann ich nicht urteilen, wie es in betr. der erwähnten andern Verschiedenheiten, spez. des Canalsystems, bei dem VANHöFFEn'schen Exemplar steht.

Von Mastigias nahe stehenden Gattungen ist zunächst Desmostoma zu erwähnen. Man könnte sich fragen, ob der Unterschied dieser VAnHöffes'schen Gattung: ein Bündel Peitschen- 
filamente im Centrum der Subumbrella, nicht ein etwas unsicherer und nach Analogie mit Cassiopcia etc. nur ein sexueller wäre. VAxßöfFEx selbst erwähnt, dass er bei einem Exemplar nur S Filamente fand, und bildet auch bei Mastigias (I 888, Taf. V, Fig. 3) solche Filamente, wenn auch in geringerer Zahl, ab. Es ist hiervon, wie fur Eucrambessa die Auffindung neuen Materials zu wünschen. Noch mehr gilt dies für eine Gattung oder ganze Gruppe, die ich ebenfalls für hierzu gehörig erachte, nämlich für die Monorhiza hacckeli Haackes, die Psendorhiza aurosa Lendenfeld's, aus der Letzterer eine besondere Familie: Chaunostomidae, (Rhizost. mit offenem Mund) gemacht hat und zu der MAyer eine neue Art Ps. thocambani hinzugefugt hat. Alle 3 beschriebenen Formen haben die grossen Gallertendkolben an den Unterarmen wie Mastigias, und umgekehrt zeichnen sich die Mastigias-formen, wie verschiedene Autoren angeben, dadurch aus, dass der Mund auffällig lange offen bleibt. Schon bei HaEckel wird ausdriicklich hervorgehoben (1897, p. 623) "dass hier die centrale Mundöffnung sich erst spät zu schliessen beginnt"; die eigenen Abbildungen HaAckes für Monorhiza und Lendenfeld's für Pseudortiza (I888, Taf. XIX, Fig. 5), [Gattungen, die iibrigens nach L. selbst zu vereinigen sind] zeigen deutlich, dass es sich nur um eine ganz kleine, offengebliebene Stelle im Centrum handelt, während sich sonst iiberall den Armkanten entlang die typischen Saugmiindchen ausgehildet haben. VANHöFFEN hat sich schon gegen die Aufstellung einer besonderen Gruppe nur auf Grund eines solchen Merkmals erklärt, zumal auch bei Stomolopluzs ein teilweise offener Mund mitunter vorkomme. Auch bei der mediterranen Rhizostoma pulmo habe ich eine centrale Oeffnung mehrmals beobachtet. Ich kann VAxHöFfEx noch weiter darin Recht geben, „dass es nicht möglich ist, aus den vorhandenen Abbildungen und Beschreibungen ein sicheres Urteil uiber die Gestalt ihrer Mundarme zu gewinnen" (I888, p. 39) aber, was heraus gelesen werden kann, lässt doch auf dreifliggelige Unterarmbildung schliessen, und andere Einzelheiten: Magenausbildung, Subgenitalporticus, Muskulatur auch die kleinen Blasenkolben, etc. stimmen zu Mastigias. Nur das periphere Canalsystem zeigt eine andere und eigenartige Bildung; es findet sich innerhalb des Ringcanals kein anastomosierendes Netz vom Magen ausgehender Canäle wie hier, sondern ausser den 8 Rhopalarcanälen noch weitere 8 adradiale, und sonst blind endigende, vom Ringcanal ausgehende Centripetalcanäle (10), von auffallend gestrecktem Verlauf (HAacke I887, Taf. 37, Lendenfeld i888, Fig. i4). [Bei Mayer's thocambani wird ebenfalls ein solches Canalsystem mit blinden Centripetalcanälen im Text erwähnt, die Abbildung zeigt dagegen (1899, Fig. 40) richtige Radiärcanäle wie bei Mastigias, und da sogar Fürbung und Fleckung mit papua übereinstimmen, so liegt es nahe seine Ps. thocambaui fuir eine Mastigias papua mit offengebliebenem. Mund zu halten. Bei HAגCkE und Lendenfeld zeigen dagegen besondere Bilder von der Subumbrella aus dieses eigenartige Canalsystem ausser Zweifel]. Wenn man also vom Canalsystem absehen wollte, so würde Pscudor/iza ganz unter Mastigias fallen, und auch M. orsini könnte bei der Gattung bleiben; so aber glaube ich, dass grerade in dem eigenartigen, in mancher Beziehung sich dem von Ly'hnor/iza nähernden Canalsystem von Pseudorhiza ein Beweis für die systematische Wichtigkeit desselben liegt. Und wenn Pseudortiza als besondere, allerdings hierher gehörige Gattung bestehen kann, so muss auch für $M$. orsini eine eigene Gattung angeschlossen werden, wenn sie nicht zu Crambessa zählt, und ferner muss Crambessa palmipes aus $C_{r}$. ausscheiden (s. p. 53). 
d) Rh. scapulata (Vanhöffen) (mit Schulterkrausen).

Die Rhizostomen mit Schulterkrausen, wie sie VArnöffex zusammengefasst hat, enthalten nur wenige Gattungen, vereinigen jedoch zwei Gruppen: Stomolophiden und Rhizostomiden (Eupilemiden Hckl.), die bei C LAUS und HAECrel zwei verschiedenen Familien resp. Unterfamilien angehören. Da bei den Merkmalen der ersteren neben den verwachsenen Armen eine dichotomische Verzweigung angeführt wird, während die letzteren typisch dreiflugglige Unterarme aufweisen, so könnte die Frage entstehen, ob die Gruppe der scapulata eine einheitliche ist, oder ob die Schulterkrausen eine von zwei Seiten erworbene Bildung darstellen. Das ist bei solch eigentümlichen Anhängen von vornherein unwahrscheinlich; zudem sind dieselben in beiden Fällen gleich gestaltet, an gleicher Stelle angebracht und in 2 mal \& Paaren verteilt, sodass sie nur durch heraufgerückte Teile von dorsalen resp. abaxialen Krausen erklärt werden können (s. HAECKEL, p. 582 und hier p. 67). Es wird also wohl die Armform von Stomolophus der Verzweigung nach mit der tripteren von Rhizostomen übereinstimmen und nur durch die gedrungene Gestalt, wie bei Rhizostomen mehrfach, eine andere Verzweigungsart vorgetäuscht werden. Die Abbildungen, die VANHöffen für Stomolophus giebt, weisen ebenfalls darauf hin. Auch das Canalsystem und andere noch bei der allgemeinen Einteilung der Rhizostomen (s. u.) zu erörternden Merkmale stimmen in beiden Fällen überein, so dass die. Gruppe wohl als eine natirliche anzusehen ist.

Dass sie den tripteren und trigonen Formen VAxhöffEx's viel näher steht, wie den dichotomen oder pinnaten, ist kein Grund gegen ihre separate Aufstellung. Die Verhältnisse der einzelnen Gruppen untereinander sollen nach Beschreibung sämmtlicher Formen ihre Erledigung finden.

\section{Rhopilema Haeckel is79.}

Rhizostome mit Schulterkrausen, freien, dreiflügeligen Unterarmen, die sowohl am Ende wie auf den Flügeln Gallertkolben tragen.

Die Gattung wurde von HAECKEL mit den obigen Merkmalen aufgestellt, jedoch von VANHÖFFEN wieder eingezogen und zu Rhizostoma (Pilema HAECKEL) gestellt, weil nach seiner Ansicht des Auftreten seitlicher Kolben neben den Terminalkolben, ebenso wie bei Eucrambessa gegeniiber Mastigias, kein Gattungs- sondern nur ein Altersunterschied ist (I 888, p. 33). Dass bei Crambessiden doch Unterschiede zwischen den seitlichen, dort allgemein vorkommenden Kolben und den Endkolben bestehen und darum Eucrambessa wohl beibehalten werden kann, habe ich oben bereits erörtert (p. 62). Hier macht Kishinouye darauf aufmerksam, dass man unterscheiden muss ,between the medusae with distinct terminal appendages and those which lack them. In the latter there is no marked difference between the terminal and lateral appendages ( 1899, p. 208) neither in shape nor in form".

Ich folge ihm daher in der Beibehaltung der Gattung Rhopilema, nicht aber in der Aufstellung einer neuen Art: wermucos ; diese ist wohl mit der neuen hispidum VANHöfFen identisch, die letzterer Autor bei seiner Vereinigung beider Genera zu Rhizostoma stellen musste. Die 
Unterschiede dieser Art von den in Betracht kommenden Arten Rhizostona clavigerum und Rhopilema rhopalophora, die besonders im Fehlen der Peitschenfilamente bei letzterer liegen, hat VANHÖFFEN bereits betont. Eine weitere neue Art KishinouxE's, R/. csculenta, die essbare Meduse Japans, unterscheidet sich ausser den Kolben durch zweierlei Armanhänge: Filamente und lange, nicht contractile Kolben, sowie durch die dicke Schirmgallerte u.a. (s. Kishinouye I 899); von hispidum dagegen ist die vorliegende Art nicht zu trennen, und was sie allenfalls von der VANiöFfEn'schen Beschreibung noch' verschieden erscheinen liesse, erklärt sich durch Altersunterschiede (s. Messungen). Alle 3 Arten. rhopalophora, hispidum, csculenta gehören dem indopazifischen Gebiet in verschiedener Ausdehnung an.

I. Rhopilema hispidum Vanh. (Taf. IX, Fig. 78, 79, 80, 8I).

Rhizostoma hispidum Vanhöffen ISSS.

Rhopilena verrucosa Kishinouye I899.

Artdiagnose bei Vanhöffen rSSS.

Makassar. I Exemplar von etwa $25 \mathrm{~cm}$. Schirmdurchmesser.

Die Exumbrella ist, so viel an dem schlaffen Exemplar zu erkennen ist, nur mässig gewölbt; sie ist mit körnigen, spitz endenden Warzen dicht besetzt und fühlt sich am Formolexemplar wie Selachierhaut an.

Die Gallertschicht ist überall sehr dünn; ihre Dicke beträgt sogar in der Mitte des Schirms nur wenige Millimeter; an den Seiten, in den Krausen und Unterarmen wird sie sogar zu einer membranösen Platte verringert.

Die Armscheibe ist von typischer Gestalt; die Eingänge in die Subgenitalhöhlung sind ungefähr so breit wie die trennenden Pfeiler. Die 4 Höhlen selbst bilden keinen ganz einheitlichen Genitalraum, wie bei Mastigias und Crambessa, sind jedoch auch nicht vollkommen getrennt, wie bei Cassiopicia, sondern communicieren in etwas unregelmässiger IVeise untereinander, indem die 4 trennenden perradialen Leisten nach dem Centrum zu schwinden, (nicht gleichmässig in jedem Quadranten) und so zu coulissenartigen Septen werden. Dies Verhalten scheint aber erst im vorgerückten Entwicklungsstadium platzzugreifen, im Gegensatz zu den Formen mit grossem, durchgängigem Subgenitalsaal, wo schon auf dem jugendlichen Stadium (von I-2 cm.) keine Spur einer Scheidewand mehr vorhanden ist.

Die Arme sind wegen der Dünne der stützenden Gallerte äusserst schlaffe Gebilde. Die Länge vom Ansatz der Armscheibe bis zur Spitze des Unterarms beträgt hier IS cm.; vom Schulter-Krausenansatz bis zum Armende kommen davon $12 \mathrm{~cm}$. Die Oberarme sind verwachsen, die Unterarme hängen frei herab. Sie zeigen die typisch-dreifligelige Form, indem sie vom Rücken (abaxial) aus in ihrer ganzen Ausdehnung auseinander geklappt werden können (Fig. 79), sodass die in natürlicher Haltung aneinanderliegenden inneren Gallertfächen zum Vorschein kommen. Dieselben sind frei ron Krausenbesatz, weil hier die Krausenreihen sich erst am Distalende einander nähern; auf den äusseren Flächen jedoch greifen diese abaxialen Krausenreihen nach der axialen über, wodurch dann hier ein allseitiger Krausenbesatz erscheint (Fig. 78). Die Form ist gedrungen, sodass das Profil der Unterarme, abgesehen von verlängerten 
Zipfeln, in der dorsalen Ecke die Gestalt eines gleichseitigen Dreiecks hat. Diese Zipfel entstehen dadurch, dass sich jede der beiden abaxialen Kanten an ihrem oberen Ende noch einmal zweigabelt, und dadurch im kleinen wiederum zweì krausentragrende Kanten und zwei innere aneinanderliegrende krausenfreie Gallertfl̈chen gebildet werden (Fig. 79, z!). Auch kann sich die Gablelspaltung mehrfach wiederholen. Für die Auffassung der Armform und den Vergleich mit dichotomen Formen ist dieser Process nicht ohne Bedeutung. Am Ende der Unterarme sitzt ein massiver Gallertknopf mit kurzem Stiel; ähnliche Knöpfe, die ebenfalls im Verhältnis zur blattartig dünnen Armgallerte sehr stark und fest erscheinen, kommen an den Seiten del Arme hervor. Kleinere Saugkölbchen oder Peitschenfilamente fehlen dagegen den Unterarmen vollkommen.

Die Schulterkrausen stehen in der bekannten Weise in 8 Paaren, ihre concave gallertige Seite nach unten, ihre convexe krausentragende nach oben gerichtet (Fig. 78 scap). Ein entodermaler Hauptcanal mit entsprechenden Seitenästen ist bis fast an ihr Ende zu verfolgen; an Grösse stehen sie den Unterarmen nicht nach, und die langen, gruppenweise stehenden Peitschenfilamente, die sie im Gegensatze zu diesen besitzen, hängen noch über das Unterarmende herab. Distal ist jede der I6 Krausen noch einmal in 2 Läppchen gespalten, ganz ähnlich wie das proximale Ende der Abaxialkrausen der Unterarme.

Das Canalsystem zeigt 4 perradiale und 4 interradiale, und ausserdem 8 adradiale, den Perradien etwas genäherte Canäle. Alle i6, nicht nur die 8 rhopalaren, lassen sich bis zum Schirmrand verfolgen. In einiger Entfernung vom Magen gehen von den Canälen, auffallend senkrecht zu ihrem Verlauf, Seitenäste ab (fig. 8I), die zu einem Anastomosennetz sich vereinigen. Proximal geht dies etwas weitmaschiger in Form der bekannten Arcaden hinauf, distal wird es allmählich enger bis zum Schirmrand und dessen Lappen. Ein Ringcanal ist nicht vorhanden; laut HAECKEL soll derselbe secundär verschwinden; doch ist hier in der Verlaufstendenz der Canäle keine Spur mehr davon wahrzunehmen.

Die Muskulatur der Subumbrella zeigt i. G. ringförmige Anordnung und ist sehr weit proximal ausgedehnt; doch ist sie durch alle i 6 Canäle auf ein breites Stiick unterbrochen, so dass 16 einzelne radiäre Felder entstehen. Diese bilden Dreiecke mit der abgestumpften Spitze nach dem Magen zu, und dadurch bleiben dazwischen Dreiecke mit der Spitze nach dem Schirmrand iibrig (Fig. 8I). Diese letzteren bilden den I6-strahligen charakteristischen Stern auf der Subumbrella; dessen Zeichnung und Durchschimmern kommt also nicht durch die geringere Verzweigung des Canalsystems, sondern durch das Freibleiben von Muskulaturlage zu Stande.

Der Schirmrand zeigt auffallend kleine Rhopalarlappen, zwischen je zweien stehen 8 fast gleich grosse abgerundete Velarlappen, dazwischen kommen noch einige wenige kleinere, nachträglich eingreschobene vor. Im typischen Fall wird die Gesammtzahl $\delta \times(2+8)=8$ o betragen.

Die Rhopalien sind wie bei der Gattung Rhizostoma selbst gestaltet, zeigen eine sehr gut entwickelte äussere Grube mit radiären Falten, am Kolben die üblichen Differenzierungen, jedoch keinen P'igmentfleck, (wie Hesse und Schewianoff richtig gegen Emaer angeben). 
c) Rh. lorifera Vanhöffen.

(Fam. Leptobrachidae Claus).

Rhizostomen mit sehr verlängerten, riemenförmigen Unterarmen und teilweise verwachsenen Oberarmen.

Genus Thysanostoma. L. Ag.

Unterarmflügel bis zum distalen Ende mit Saugkausen besetzt.

I. Thysanostoma thysanura Hckl. (Taf. X, Fig. 93, 94).

Stat. 53. I Exemplar von ungefähr $12 \mathrm{~cm}$. Durchmesser.

Die betr. Art ist nach HaEckel noch ausserdem von L. S. Schultze sorgfältig beschrieben worden ( $\mathrm{r} 898$, p. 448). Hier sollen nur einige Bemerkungen über die Mundarme Platz finden, um deren Ableitung von den tripteren Formen und damit später die Stellung der loriferen im System zu begründen.

Die Mundarme sind fast gleichlang, etwa $16-18 \mathrm{~cm}$., der Oberarmteil beträgt nur etwa $\mathrm{I}^{1} / \mathrm{cm}$; d der Unterarm ist sehr schlaff und platt zusammen gelegt; dennoch erscheint er bei genauerem Zusehen deutlich dreiflügelig, in der ganzen Länge aufklappbar, nur sind die Flügel messerartig dün und der axiale Gallertteil sehr zuruickgebildet (s. Querschnitt Fig. $94 a$ und b), so dass im unteren Teil eigentlich nur zwei Flügel vorhanden sind, die sich an der axialen Krausenkante vereinigen. Im oberen Teil ist aber die triptere Form ganz ähnlich wie bei Mastigias und bei Crambione in typischer Weise zu erkennen. Ferner sitzen hier seitliche secundäre Flügel auf (Fig. 93z!), ähnlich wie es HAECrEL auch fir Himantostoma abbildet. Dadurch ist der Krausenbesatz hier allseitig, ebenso wie im ganzen ersten Drittel des Unterarms, wo die Krausen vollständig von den Kanten auf die Flächen übergreifen, so dass man von trigoner Form im Sinne VAxHöffex's sprechen könnte. Im mittleren Drittel werden die Krausen sichtlich spärlicher, nur noch auf die Kanten beschränkt, so dass hier die triptere Ausprägung im Sinne VAxhöfrex's besteht; im letzten Drittel greifen die Krausen wieder allseitig über wie bei Crambessiden. Die Enden meines Exemplares sind wohl erhalten, nicht abgerissen und zeigen keine Spur eines gallertigen Terminalknopfes. Besondere Anhänge sind zwischen den Armkrausen nicht erkennbar; auf der centralen Armscheibe nur vereinzelte schwache Fädén (das Tier ist Männchen).

Die Exumbrella zeigt eine deutliche Täfelung; deren Polygone sind im Centrum etwas grösser, an der Peripherie kleiner, stets hinter der Breite der Randlappen merklich zuriickbleibend.

Die Randlappen sind in Zahl und auch Gestalt verschieden. Von HaEcker wird die Zahl zur Abtrennung der sehr problematischen Art Th. melitaed benutzt; von Kishiroure wird eine typische Randlappenzahl bei Th, bestritten, von L. S. Schultze dagregen wieder als Merkmal hervorgeholt. Gerade nach dessen eigener sorgfältiger Beschreibung ( 898, p. 448) kann 
ich mich davon nicht überzeugen. Die Lappen sind auch hier pro Octant durch Alter, Teilung und Einschub in verschiedener Zahl und Grösse vorhanden. Es stehen ihrer 6-io zwischen je zwei Rhopalien; manche davon sind doppelt und dreifach so breit wie andere (schon geteilte). Der Form nach sind sie rhombisch abgerundet; die Rhopalarlappen etwas länger und spitz.

Der Subgenitalsaal ist ein grosser, einheitlicher, niedriger Raum mit kolossal weiten Oeffnungen. Die perradialen Armpfeiler sind nur etwa I $\mathrm{cm}$. dick, die interradialen Oeffnungen uiber $4 \mathrm{~cm}$. weit.

Die Subumbrellar-muskulatur besteht nur aus Ringfasern, die nach der Peripherie zu stärker und kontinuirlicher werden und dort durch besonders starke Entwicklung der Stützleisten ausgezeichnet sind, wie auch L. S. Schultze angiebt.

Der Beschreibung der Armscheibe ist nichts hinzuzufuigen; die Oberarme erscheinen hier nur wenig verwachsen.

Das Canalsystem ist ausgezeichnet durch etwas grösseres Kaliber der Rhopalarcanäle und einen ziemlich weit vom Schirmrand entfernten Ringcanal; im übrigen bildet es ein unregelmässiges Netzwerk, teilweise mit Blindsäcken, das jenseits des Ringcanals plötzlich selır viel enger wird. (s. Haeckel's und Kishinouye's Figuren i 895 \%).

Die Gonaden sind von L. S. Schultze genau, auch auf Schnitten, untersucht worden (1898, p. 45I) und zeigen die bekannten Verhältnisse.

Ausser Th. thysamura gehören zur Gattung noch Th. melitaca (eigentlich lessoni, aber von HAECKEL wegen der ungenügenden Beschreibung LESSON's umgetauft) und eine neue Th. denscrispum Kishinouye. Ob molitaca verschieden ist von thy'samura, erscheint nach den obigen Bemerkungen iber die Randlappen und nach der übrigen mangelhaften Kennzeichnung mehr wie zweifelhaft. Sie ist zwar die älteste und Stammart der Gattung; aber doch ist ihre Streichung zu Gunsten von thysanostoma, vorzunehmen (nicht umgekehrt nach dem Prioritätsgesetz, zu verfahren, weil in diesem Fall, die Beschreibung von melitaca resp. lessoni durchaus unzureichend für die Kennzeichnung war). Die Art Kishinouve's scheint mir nur ein Jugendstadium zu sein; ihre einzigen Unterschiede sind die gewölbtere Umbrella und die viel kiirzeren MIundarme, wodurch sie, wie er sagt, sich dem Genus Crambessa nähert. L. S. Schultze helot bereits hervor, dass die Arme der Himantostomiden erst nach und nach wachsen und die jungen Thysanostoma darum eine Annäherung an die Crambessiden erkennen lassen (ISg8, p. 452). Da nun Kisłnnouye nur ein einziges Exemplar und zwar von $4 \mathrm{~cm}$. Durchmesser gehabt hat, die erwachsenen aber das $4-5$ fache dieses Durchmessers erreichen, da ferner die gewölbtere Umbrella auch ein Anzeichen von nicht vollendetem Wachstum ist, so ist denscripsum ebenfalls zu thy'sanostoma zu rechnen.

Zur Gruppe lorifera gehören noch die Genera Himantostoma (L. Ag), durch einen terminalen gallertigen Anhang an den Armen ausgezeichnet mit 3 (??) Arten, und ferner Leptobrachic (Brandt) und Lcomera (Hckl), ebenfalls mit Endknopf, aber mit Saugkrausen nur am Ende, dagegen im mittleren Drittel kahlen Nundarmen. [Wenn dies Verhalten normal ist, so erscheint es durch Formen wie Thy'sanostoma schon vorbereitet, wo, wie oben erwähnt, die Krausen im mittleren Drittel viel schwächer entwickelt sind und nur an den Kanten sitzen; 
vielleicht liegt es jedoch nur am Erhaltungszustand der hierher gerechneten Arten]. Leptobrachia und Lconura sollen durch eine centrale Zottenrosette unterschieden sein; den Mangel der letzteren bei Lcptobrachia, der zudem nur aus einer Abbildung von is 82 geschlossen wird, kann ich aber nur als einem individuellen, (oder höchstens sexuellen) Unterschied gelten lassen (s. Cassiopeia). Leomura ist daher als Gattung nicht aufrecht zu erhalten.

Himantostoma L. Ag. I 862.

Sens. emend. Hckl.

Unterarme mit einem saugkrausenfreien Endabschnitt.

1. Himantostoma flagellatum Hckl 1879. (Taf. X, Fig. 87-92. Taf. XI, Fig. IOI).

Stat. 317. 2 Exemplare von 12 und $8 \mathrm{~cm}$. Schirmdurchmesser.

Endabschnitt der Unterarme nicht kolben-, sondern peitschenförmig. Aehnliche Filamente auch meist auf den Unterarmen verteilt. Arme im erwachsenen Zustand kaum 1 änger als der Schirmdurchmesser. Ostien etwa doppelt so breit als die Pfeiler. 8 Velarlappen $z w i s c h e n 2$ kleinen spitzen Ocularlappen.

Die vorliegenden Exemplare sind kleiner als bei HAECKEL für die Species angegeben ist und erweisen sich auch dadurch, dass ihre Arme noch nicht die extreme Streckung der loriferen erreicht haben, ebenso wie das eben erwähnte Thysanostoma-exemplar Kishinouve's als nicht erwachsene Stadien. Die Armform, der Krausenbesatz, die Verwachsung der Oberarme und andere Merkmale zeugen aber unzweifelhaft für ihre Zugehörigkeit. Immerhin erreichen auch schon bei diesen jüngeren Exemplaren die Arme gegenuiber den Verhältnissen anderer Rhizostomiden eine sehr beträchtliche Länge (s. Fig. 87), und sie scheinen ja laut HaEckeL's Diagnose bei der vorliegenden Art überhaupt nicht so extrem lang zu werden wie bei H. lorifer $\alpha$ und andern Arten der Gruppe. Dadurch, sowie durch die Endanhänge (oder je nach Auffassung: Endabschnitte) der Arme unterscheidet sich H. flagellatum sehr merklich von H. lorifera und lesucuri Es kommen weitere Unterschiede dazu, so dass es fraglich erscheint, ob nicht eine besondere Gattung zu schaffen wäre. Deshalb und weil die betreffende Art bei HaEcres selbst, der sie aufgestellt hat, gegenüber lorifera nur sehr kurz wegkommt, erscheint eine besondere Beschreibung hier gerechtfertigt, besonders auch der Arme, die eine Ableitung der loriferen Form von der typischen tripteren auf diesem jüngeren Stadium deutlich gestatten. H. lorifora ist zudem mehrfach wiedergefunden worden, $H$. flagellatum jedoch bis jetzt noch nicht.

Der Schirm ist flach gewölbt; die Gallerte ist dick und von zäher Consistenz. Sie erscheint an beiden Exemplaren nicht durchsichtig, sondern porzellanartig weiss. Die Exumbrella ist äusserst fein gekörnelt und resp. punctirt und zeigt am Rand auf der weissen Fläche hellbraune Tupfen, die auf den Randlappen deutlicher und zahlreicher werden (Fig. S9).

Die Armscheibe ist 8 eckig; die Subgenitalostien sind grösser als die trennenden Pfeiler, doch ist der Grössenunterschied lange nicht so beträchtlich wie bei andern Himantostomiden. Schon bei ihrem Ansatz an der Subumbrella zeigen sich die Pfeiler zweigeteilt; zwei 
Gallertbänder, den Oberarmbasen entsprechend, ziehen bogenförmig herauf (Fig. 88) und sind von innen durch eine stärkere quergewulstete Gallertmembran verbunden, die auch interradial bis heruber zum andern Pfeiler zieht und das Subgenitalostium überbrückt. Diese Membran ist der von HAECKEL beschriebene, die Oberarme verbindende Halskragen, das Patagium (Fig. SS pat). Da auch hier durch Conservierung alle diese Teile lederartig zusammengezogen waren, so kann ich nur die genauere Darstellung von L. S. Schultze (1898, p. 447) für lorifera bestätigen. Unterhalb dieses schwimmhautartigen, die Oberarmbasen nur zum Teil verbindenden Saums sind die Oberarme dann wieder auf ein sehr kurzes Stiick frei (Fig. 9I br.); dann beginnen sofort die Flügel der Unterarme (Fig. 9 I z). Auffallig ist, dass die Oberarme, die viel weiter peripher beginnen, sich centralwärts so sehr einander nähern, gerade wie wenn dieses Patagium eine einschnürende Wirkung hätte. Unterhalb dieser taillenartigen Einschnürung neigen dann die freien Armteile wieder etwas mehr nach aussen.

Der Unterarm zeigt noch deutlich die Zurückfuihrung auf die dreiflüglige Gestalt; er ist an der abaxialen Seite aufklappbar und lässt deutlich zwei Dorsalflügel erkennen, die durch einen tiefen Einschnitt getrennt sind und nur an den Kanten die Krausen tragen. Der Querschnitt des Armes entspricht also hier noch der für Thysanostoma gezeichneten und fur alle tripteren geltenden Form (Fig. 92a, 94a). Eine Strecke unterhalb jedoch treten die Fliggel gegenüber dem verbindenden Gallertteil zurück, der Querschnitt wird einfach dreieckig, so dass die Krausen den Arm jetzt allseitig umhïllen (Fig. 92b). Auf solche Weise ist der weitaus grösste Teil des Unterarms zusammengesetzt, der sich allmählich verschmälert, bis er in einen krausenlosen Endabschnitt übergeht. Es frägt sich, ob man diesen Endteil nicht als einen einfachen Peitschenanhang auffassen soll, da ja ähnliche, kaum kürzere und dünnere Peitschenfilamente auch sonst auf den Armen sitzen. Doch ist der Unterarm hier bereits derart verschmälert, dass sein Endabschnitt kaum dicker sein wird als eine gewöhnliche Peitsche. Der Krausenbesatz erscheint äusserst dicht, wie eine wollige Umhüllung des Unterarms, ohne dass sich die abgehenden Aestchen von aussen erkennen liessen; andere Anhänge als die erwähnten Peitschen, sind nicht zu erkennen. Am grösseren der beiden Exemplare betrug die Länge des Unterarms etwa 8, clie Länge des freien Oberarmteils etwa $I^{1} / 2 \mathrm{~cm}$.

Der Magen ist von breiter Form. Die Schenkel des Kreuzes sind ungefähr ebenso lang wie die Stelle in der sie zusammenkommen; auch hierdurch ist ein bemerkenswerter Unterschied von II. Lorifer $x$ und anderen Himantostomiden gegeben, bei denen eine extreme Streckung der Kreuzform die Regel ist (s. HAEckel, Taf. XXXVIII und Taf. XXXIX).

Das Canalsystem zeigt \& Rhopalarcanäle deutlich durch Caliber und Verlauf ausgezeichnet. Ausserdem gehen noch andere Zwischencanäle vom. Magen ab und bilden ein Netzwerk in der Subumbrella. Der vollständige Zusammenhang dieses Netzwerks mit dem Magen, sowie die genaue Zahl der Zwischencanäle konnte nicht ermittelt werden. Ohne deutlich erkennbaren Ringcanal wird das zuerst weite Maschenwerk an der Peripherie enger und geht am Schirmrand mit entsprechenden Maschen in die Lappen hinein, Ein besonderer Bogencanal in den einzelnen Lappen ist nicht vorhanden; nur in den Rhopalarlappen erscheinen hier noch die einfachen V'erhältnisse.

Die Muskulatur bilclet eine starke Ringfaserschicht, die nach der Peripherie zu 
allmählich abnimmt und auch centralwärts in den Perradien und Interradien ziemlich gleichmässig abschneidet. Durch die Radialcanäle erscheint ihr Verlauf nicht unterbrochen; höchstens ist noch, perradial mehr als interradial, eine Verdünnung der Muskelsubstanz wahrzunehmen.

Die Velarlappen sind (durch Contraction?) auffallend gewölbt und viel länger als die Rhopalarlappen. Letztere bilden nur zwei kleine ausgespreizte Dreiecke zur Seite des Sinnesorgans, die kaum $2 / 3$ der Länge der Velarlappen erreichen. Deren Zahl beträgt typisch 8, ihre Gestalt ist an den vorliegenden Exemplaren kielförmig. Hie und da sind eingeschobene, noch schmälere und kleinere Läppchen zwischen den 8 typischen zu erkennen.

Die Rhopalien haben eine gut entwickelte äussere Sinnesgrube, wie Crambessa und Rhizostoma selbst, mit radiären Falten. Der Kolben selbst ist von auffallend gedrungener Form mit mächtigem Bulbus und einer Endschuppe die breiter wie lang ist (Fig. IOI). Von den Bildungen bei Rhizostoma und Crambessa ist er auch durch den unpaaren, im Aufsichtsbild hufeisenförmigen Ocellus verschieden; in seinen sonstigen Differenzierungen aber gleich.

Die Gonaden sind an den betreffenden Exemplaren, trotzdem sie nicht ausgewachsen sind, bereits in der typischen Form stark gefalteter interradialer Hufeisen entwickelt. Dass sie zum Ostium bruchsackartig heraus ragen, erklärt sich durch die erwähnte Contraction der ganzen Stuicke. Es ist dies auch wohl bei anderen Formen z.B. Lychnorhiza, wo es von HAECKEL angefuhrt, von den Nachfolgern nicht gesehen wurde, nur durch die Fixierung bedingt. 
Uebersicht der wichtigsten Rhizostomeengattungen.

\begin{tabular}{|c|c|c|c|}
\hline & ARMFORM UND ANIIALNGF. & SUbGentTalostien. & CANalsystem. \\
\hline Cepher. & $\begin{array}{l}\text { zweilliiglig (dichotom). } \\
\text { (seitl. gefiedert), mit Peit- } \\
\text { achenfilamenten. }\end{array}$ & eng, trichterförmig. & $\begin{array}{l}\text { S gesonderte Rhopalar- } \\
\text { canäle, dazw. zahlreiche ad- } \\
\text { radiale, die alle zusammen } \\
\text { ein peripher allmähl.engeres } \\
\text { Netz bilden, ohne Ringcanal. }\end{array}$ \\
\hline Cotylorhiza. & $\begin{array}{l}\text { dichotom, mit langen ge- } \\
\text { stielten Saugnäpfen. }\end{array}$ & eng, trichterförmig. & $\begin{array}{l}\text { Zablreiche gleichmässige } \\
\text { Radiärcanäle, die ein Netz } \\
\text { wie oben bilden, ohne Ring- } \\
\text { canal. }\end{array}$ \\
\hline Netrostomet. & $\begin{array}{l}\text { dichotom, mit wiederholter } \\
\text { Gabelung, ohne Filamente, } \\
\text { mit kleinen Kolbenblasen. }\end{array}$ & eng, trichterformig. & $\begin{array}{l}\text { S Rhopalar-, } 3 \times S \text { ad- } \\
\text { radiale Canäle, die ein Netz } \\
\text { wie oben bilden, ohne Ring- } \\
\text { canal. }\end{array}$ \\
\hline Cassiopeia. & $\begin{array}{l}\text { gefiedert, mit kleinen } \\
\text { Blasenkolben. }\end{array}$ & eng, kreisrund. & $\begin{array}{l}\text { I } 6 \text { rhopalare, } 16 \text { und mehr } \\
\text { adradiale Canäle, die ein } \\
\text { intmeres und äusseres Anas- } \\
\text { tomosennetz bilden. /wei } \\
\text { Ringcanale (innerer und } \\
\text { äusserer). }\end{array}$ \\
\hline Rhizostoma. & $\begin{array}{l}\text { dreifliglig, mit beson- } \\
\text { dereu Schulterkrausen, mit } \\
\text { Endkolben. }\end{array}$ & $\begin{array}{l}\text { Ostien querge- } \\
\text { schlitzt, durch Kilap- } \\
\text { pen verengert. }\end{array}$ & $\begin{array}{l}\text { S rhopalare, } 8 \text { adradiale } \\
\text { Canäle, die alle } 16 \text { zum } \\
\text { Schirmrand gehen. Enges } \\
\text { ausseres Anastomosennetz, } \\
\text { zwischen } 2 \text { Radiärcanälen } \\
\text { centralwärts blind aufstei- } \\
\text { gend. }\end{array}$ \\
\hline Stomolophus. & $\begin{array}{l}\text { Verzweigung? Arme } \\
\text { verwachsen, mit Schulter- } \\
\text { krausen. }\end{array}$ & & wie oben. \\
\hline Toxoclytus. & $\begin{array}{l}\text { dreilliglig, ohne Kolben, } \\
\text { Filamente und sonstige An- } \\
\text { hïnge. }\end{array}$ & $\begin{array}{l}\text { Ostien quer, so } \\
\text { gross wie Pfeiler; } \\
\text { durch Kilappenver- } \\
\text { engert und geteilt. }\end{array}$ & $\begin{array}{l}\text { S rhopalare, } S \text { adradiale } \\
\text { Canäle; ausserdem blinde } \\
\text { Centripetalcanäle, Ringcanal } \\
\text { u. intracirculäres Gefissnetz. }\end{array}$ \\
\hline Psendorkiza. & dreifliglig, mit Endkolben. & $\begin{array}{l}\text { Ostien weit; grösser } \\
\text { als Pfeiler. }\end{array}$ & $\begin{array}{l}\text { S rhopalare, } 8 \text { adradiale } \\
\text { Canäle, die ersteren zum } \\
\text { Schirmrand, die anderen nur } \\
\text { zum Ringcanal reichend, } \\
\text { ausserhalb des Rings enges } \\
\text { Netz, innerhalb ro blinde } \\
\text { gerade Centripetalcanile. }\end{array}$ \\
\hline Lycknorhizn. & $\begin{array}{l}\text { dreituiglig und seitlich } \\
\text { gelappt, ohne Kolben, aber } \\
\text { mit lilamenten. }\end{array}$ & $\begin{array}{l}\text { Ostien weit: } \\
\text { grosser als Pfeiler. }\end{array}$ & $\begin{array}{l}\text { S rhopalare, } S \text { adradiale } \\
\text { Canäle, die ersteren zun } \\
\text { Schirmrand, die letzteren } \\
\text { nur zum Ringcanal gehend. } \\
\text { Ausserhalb dieses engeres } \\
\text { Netz, innerhalb einzelne } \\
\text { breite, teilweise verbundene } \\
\text { Centripetalcanäle. }\end{array}$ \\
\hline
\end{tabular}

MUskulatur. Riromalien.

starke Radialzüge, schwache Circularzone am Rand.

restreclit, ohne Ocellus, ohne äussere Sinnesgrube.

starke Radialzïge dicht nebeneinander.

starke Radialzüge in Abständen, schwache Circulärzone am Kand.

16 Fiederarcaden; ausserdem centrale und periphere circuläre Zone und radiale Stränge in einzelnen Radien.

circuläre Züge weit centrälwarts reichend, durch 16 Kadiürcanäle unterbrochen, so dass 16 dreieckige Felder entstehen.

wie oben.

circuläre Lace, ohne radiäre Unterbrechung.

circuläre Lage kaum unterbrochen.

circuläre Lage ohne radiäre Unterbrechung. mit Ocellus mit schwach ausgebildeter äusserer sinnesgrube ohne Radiarfalten.

gestreckt, ohne Ocellus und ohne

sehr gestreckt, ohne Ocellus und ohne äussere Grube.

kurz, mit Ocellus und kleiner äusserer sinnesgrube.

ohne Ocellus, aber mit ausserer gefaltetel Sinnesgrube.

mit Ocellus?, und mit äusserer, gut entwickelter radiär gefalteter Sinnesgrube. äussere Grube. 


\begin{tabular}{|c|c|c|c|c|c|}
\hline & ARMFORM UND ANHAENGE. & SUBGENITALOSTIEN. & CANALSYSTFM. & Muskulatur. & RHOPALIEN. \\
\hline Crambione. & $\begin{array}{l}\text { dreiflïglig, mit seitlicher } \\
\text { Lappung. Mit kleinen Kiol- } \\
\text { ben und grossen Peitschen- } \\
\text { flamenten. }\end{array}$ & $\begin{array}{l}\text { Ostien quer ge } \\
\text { schlitzt, kleiner als } \\
\text { Pfeiler durch Klap- } \\
\text { pen verengert. }\end{array}$ & $\begin{array}{l}\text { S rhopalare, } S \text { adradiale } \\
\text { Canäle; erstere zum Schirm- } \\
\text { rand, letztere nur zum Ring- } \\
\text { canal gehend. Ausserhalb } \\
\text { dessen enges, innerhalb } \\
\text { blind endigendes, nicht nit } \\
\text { den Radiärcanälen verbun- } \\
\text { denes Netz. }\end{array}$ & $\begin{array}{l}\text { circuläre Lage } \\
\text { ohne radiäre Lnter- } \\
\text { brechung. }\end{array}$ & $\begin{array}{l}\text { mit paarigen Augen- } \\
\text { Hecken und mit } \\
\text { äusserer, grosser, } \\
\text { radiär gefalteter } \\
\text { Sinnesgrube. }\end{array}$ \\
\hline Crambessa. & $\begin{array}{l}\text { dreifliiglig; ohne Anhänge; } \\
\text { keine Peitschenfilamente. }\end{array}$ & $\begin{array}{l}\text { Ostien weite quere } \\
\text { Schlitze. }\end{array}$ & $\begin{array}{l}\text { wie oben, nur dass das } \\
\text { intracirculäre Netz ausser } \\
\text { mit dem Ringcanal auch } \\
\text { noch mit den Radiärcanälen } \\
\text { anastomosiert. }\end{array}$ & $\begin{array}{l}\text { circuläre Lage, an } \\
\text { den } 8 \text { Radien kaum } \\
\text { unterbrochen. }\end{array}$ & $\begin{array}{l}\text { ohne Pigmentfleck } \\
\text { aber mit grosser, } \\
\text { radiär gefalteter } \\
\text { äusserer Sinnes- } \\
\text { grube. }\end{array}$ \\
\hline Iotor his.t. & $\begin{array}{l}\text { dreifiglig, mit seitlicher } \\
\text { Lappung, ohne Anhänge, } \\
\text { weder Kolben noch Fila- } \\
\text { mente. }\end{array}$ & $\begin{array}{l}\text { Ostien quer, so } \\
\text { gross wie Pfeiler; } \\
\text { durch Klappenbil- } \\
\text { dung eingeengt. }\end{array}$ & $\begin{array}{l}\text { S rhopalare, (zahli. andere?) } \\
\text { Radiärcanäle, die ein allge- } \\
\text { meines Anastomosennetz } \\
\text { mit schwachen Ringcanal } \\
\text { bilden. }\end{array}$ & $\begin{array}{l}\text { circuläre Lage ohne } \\
\text { Unterbrechung. }\end{array}$ & $\begin{array}{l}\text { äussere Grube mit } \\
\text { Radiärfalten. }\end{array}$ \\
\hline Crossostoma. & $\begin{array}{l}\text { dreifliglig, mit seitlicher } \\
\text { Lappung. Mit Kolbenblasen } \\
\text { und centraler Mundrosette. }\end{array}$ & $\begin{array}{l}\text { weite Oefinungen, } \\
\text { grösser als Pfeiler. }\end{array}$ & $\begin{array}{l}\text { Srhopalare und zahlreiche } \\
\text { adradiale Canäle, die ein äus- } \\
\text { seres engeres Netz jenseits } \\
\text { vom Ringcanal, - ein weit- } \\
\text { saschigeres diesseits des- } \\
\text { selben bilden, das auch mit } \\
\text { dem Magen anastomusisiert. }\end{array}$ & $\begin{array}{l}\text { Zwei circuläre } \\
\text { Lagen, in den } 8 \\
\text { Radien unterbro- } \\
\text { chen; interradial } \\
\text { hoch centralwärts } \\
\text { reichend. }\end{array}$ & $\begin{array}{l}\text { Ocellus? äussere } \\
\text { Sinnesgrube ohne } \\
\text { Radiärfalten. }\end{array}$ \\
\hline Mastigias. & $\begin{array}{l}\text { dreiflüglig, mit seitlicher } \\
\text { Lappung. Mit grossen End- } \\
\text { kolben und kleinen seitl. } \\
\text { Kolbenblasen. }\end{array}$ & $\begin{array}{l}\text { Ostien quer, breiter } \\
\text { als Pfeiler, aber } \\
\text { durch Pfeilerwöl- } \\
\text { bung etwas einge- } \\
\text { bogen. }\end{array}$ & $\begin{array}{l}\text { wie oben. } \\
\text { (7-9 und mehr adradiale, } \\
\text { anastomis. Canäle zwischen } \\
\text { je zwei rhopalaren). }\end{array}$ & $\begin{array}{l}\text { circuläre Lage } \\
\text { mit breiter Unter- } \\
\text { brechung in den } \mathrm{S} \\
\text { Radien, so dass } \mathrm{S} \\
\text { Bander entstehen. }\end{array}$ & $\begin{array}{l}\text { starker Ocellus, } \\
\text { schwache ïussere } \\
\text { Sinnesgrube, ohne } \\
\text { Radiärfaltung. }\end{array}$ \\
\hline Desmostoma. & $\begin{array}{l}\text { dreiflüglig, mit Endkolben } \\
\text { und kleineren Kalben und } \\
\text { ausserdem centralen Peit- } \\
\text { schenfilamenten. }\end{array}$ & $\begin{array}{l}\text { niedrige quere } \\
\text { Spalten; grösser als } \\
\text { Pfeiler; durch Gal- } \\
\text { lertklappe schein- } \\
\text { bar zweigeteilt. }\end{array}$ & wie oben. & $\begin{array}{l}\text { circuläre Lage, } \\
\text { Unterbrechung ? }\end{array}$ & -- \\
\hline Thysanostoma. & $\begin{array}{l}\text { dreifliglig, sehr stark ver- } \\
\text { längert. }\end{array}$ & $\begin{array}{l}\text { weite klaffende } \\
\text { Spalten grösser als } \\
\text { Hfeiler; nicht durch } \\
\text { Klappen verengert. }\end{array}$ & $\begin{array}{l}\text { S Rhopalarcanäle, zahlr. } \\
\text { andere, die ein unregel- } \\
\text { mässiges Netzwerk bilden, } \\
\text { das mit diesen und dem } \\
\text { Magen zusammenhängt. } \\
\text { Ringcanal vorhanden. }\end{array}$ & $\begin{array}{l}\text { circuläre Lage, } \\
\text { interradial höher } \\
\text { reichend, mit klei- } \\
\text { ner Unterbrechung } \\
\text { in den } 8 \text { Radien. }\end{array}$ & . \\
\hline Himantostoma. & $\begin{array}{l}\text { dreiflüglig, etwas verlängert, } \\
\text { mit krausenfreiem Endteil. } \\
\text { Oberarme etwasverwachsen. }\end{array}$ & $\begin{array}{l}\text { weite niedrige } \\
\text { Spalten. }\end{array}$ & -- & $\begin{array}{l}\text { circulïr mit kleiner } 1 \\
\text { Unterbrechung. }\end{array}$ & $\begin{array}{l}\text { mit Pigmentlleck, } \\
\text { und stark entwic- } \\
\text { kelter äusserer } \\
\text { Sinnesgrube mit } \\
\text { Radiärfalten. }\end{array}$ \\
\hline
\end{tabular}

dreifiuglig sehr verlängert, Lnterarme auf grössere Aus- etwas grösser als dehnung krausenfrei. Pfeiler und ausgebogen.

8 rhopalare, 8 adradiale Canäle, beide uiber den Ringcanal hinaus erkennbar. Engeres ausseres, blind en. dendes inneres Canalnetz. 


\section{Bemerkungen zum System der Rhizostomeen.}

Die von der Siboga erbeuteten Rhizostomeen weisen Vertreter aller Einzelgruppen auf, welchem der verschiedenen Systeme man auch folgen mag, und geben Anlass zum Vergleich mit zahlreichen früher und neverdings beschriebenen anderen Gattungen und Arten. Es ist darum angezeigt, auf Grund dieses Materials einiges zur so viel discutierten Systematik der formenreichen Rhizostomen beizutragen.

HAECKEL's Einteilung ( $18 \% 9$ ), die in erster Linie die Ausbildung des Subgenitalraums, in zweiter die Verteilung der Saugkrausen auf den Armen bericksichtigt und darnach in schematischer Weise $2 \times 2$ Gruppen bildet, ist bereits von Claus (I883) scharf kritisirt worden. Derselbe kommt wieder auf die Gruppierung von L. AGAssiz zurück, bringt den neueren Befunden entsprechend zahlreiche Modificationen und schärfere Umschreibungen, ohne jedoch fiir alle Gruppen durchgreifende und fü die praktische Bestimmung verwertbare Merkmale zu geben. In dieser Hinsicht ist des System VANiöffex's (I 888 ) ein wesentlicher Fortschritt. Alle Rhizostomeen werden nach der Gestalt und Verzweigungsart der Mundarme in 7 Gruppen nebeneinander gestellt. Diese entsprechen von einzelnen Umgruppierungen abgesehen i. G. den CLaus'schen Familien, aber die Bestimmung selbst ist gegenüber dem System des letzteren wesentlich erleichtert. Neuerdings hat demgegeniber L. S. Scrultze Haeckel's System zu halten versucht (1898). Dessen erstes Einteilungsprinzip, die Ausbildung des Subgenitalraums, das die Gegner als ontogenetisch wechselnd und bei sonst nahe verwandten Formen verschieden hinstellen, diurfe "nicht unterschätzt" werden, auch hätten es die Gegner selbst angewandt; das zweite Prinzip, die Saugkrausenverteilung, scheide mindestens ebenso scharf wie die Armgestalt, und von VANIIöfrex's 7 Gruppen kämen darnach 3 auf die Unicrispen, 4 auf die Multicrispen. Dagegen hat neuerdings ( I 902) VAniöffen erwidert, dass dieses Prinzip bei HaEcrel ja erst als zweites bei der Einteilung komme, nachdem der natïrliche Zusammenhang bereits rorher durch die Scheidung von Tetrademniern und Monodemniern nach dem Subgenitalraum auseinander gerissen sei, und ferner, dass er den Subgenitalranm nur aus Nangel an besseren Unterscheidungsmerkmalen bei einigen bisher nur von HAEckel gesehenen Gattungen in seiner Uebersicht benutzt habe.

Auf beide Merkmale sei auf Grund der oben beschriebenen und discutierten Formen ctwas eingegangen. Ich muss ebenso wie Claus und VAniöffen einwenden, dass der Bau des Subgenitalraums bei sonst sehr ähnlichen und offenbar nahe verwandten Formen, wie z.B. Cephaca und Cotylorhiza, Lychorhiza und Toxoclytus verschieden sein kann, und dass dies auch 
im Lauf der Ontogenese wechselt, indem bei Formen, die zuerst vier getrennte Höhlen haben, nach und nach eine Vereinigung durch Aufösung der Scheidewände eintreten kann. Dies kann bei derselben Art, sogar bei demselben Individuum in verschiedenen Radien, wie oben bei Netrostoma gezeigt wurde, verschieden sein. Dass schliesst natülich nicht aus, dass in anderen Fällen scharfe Unterscheidungen von vornherein vorhanden sind und zeitlebens erhalten bleiben, dass z.B. bei Cassiopcia stets nur vier getrennte Höhlen zu erkennen sind, bei Mastigias u. A. schon bei ganz jungen kaum $1 \mathrm{~cm}$. grossen Stadien mit noch offenem MIund ein einheitlicher Subgenitalraum besteht. Es kann also die Ausbildung des Subgenitalraums bei manchen Gruppen mit zu den Merkmalen gerechnet werden, wie bei den am einen Ende der Reihe stehenden dichotoma, oder den am anderen stehenden lorifera, oder innerhalb einer Gruppe manchmal mit zu den Gattungsmerkmalen; aber als prinzipielles Merkmal ist es der angefuhrten Bedenken wegen unmöglich zu gebrauchen, und gar als erstes angewandt, reisst es eine Reihe von nahe verwandten Formen auseinander.

Der Schultze'schen Begründung, dass, die verschiedene Ausbildung der Subgenitalregion.... erhebliche Verschiedenheiten der Armscheibe und des Gastrocanalsystems im Gefolge hat" (1898, p. 463) kann ich nicht zustimmen. Allerdings wenn an der Armscheibe äusserlich sehr weite Ostien vorhanden sind, die die Pfeiler um ein mehrfaches übertreffen, wie z. B. bei Mrastigias, oder Himantostoma dann ist es gar nicht anders möglich, als dass ein einheitlicher Saal, beinahe als Teil der Subumbrella vorhanden ist. Umgekehrt aber, können bei 4 engen runden Ostien und gleicher Configuration der Armscheibe ganz verschiedene Verhältnisse des Subgenitalraums platzgreifen; es können 4 getrennte Höhlen sein, wie bei Cassiopeia, ein teilweise Communication wie bei Netrostoma, oder eine vollständige Vereinigung wie bei Cotylorhiza. Gerade dass man sich enge Oeffnungen nur mit getrennten Subgenitalhöhlen zusammen vorkommend denken konnte, hat zu mancher falschen Bestimmung Anlass gegeben. Dass das Gastrocanalsystem von der Ausbildung der Subgenitalraums beeinflusst würde, kann ich ebensowenig finden; nur einige untergeordnete Verzweigungen in der Armscheibe selbst kann dies betreffen, aber die Versorgung der Arme selbst und namentlich das wichtige Canalsystem der Subumbrella, das auch Schultze selbst mehr für die Systematik verwandt sehen möchte, bleiben von der Configuration des Subgenitalraums ganz unberührt.

Was nun der Bau der Mundarme betrifft und das Verhältnis der Saugkrausen zu denselben, so lassen sich allerdings, wie Schultze hervorhebt, zwe i Haupttypen, nicht sieben, unterscheiden, die zudem nach HAECKEL's vergleichend-anatomischen, nach $C_{\text {LAUS' }}$ embryologischen Untersuchungen in einander überführbar sind; aus der dichotomischen resp. unicrispen Form, als der jüngern, lässt sich die dreiflüglige resp. multicrispe ableiten. Noch mehr existieren, laut Schultze, zwischen den einzelnen Gruppen VANHörfen's Uebergänge. Es soll z.B. bei Mastigias schwer zu entscheiden sein, ob wir eine fiedrige oder dreifluglige Form vor uns haben, oder bei Cassiopcia cyclobalia ein Uebergang von Fiederung zu Dichotomie bestehen. Dass solche Uebergänge „als Mängel des Systems hervortreten müssen", hat VAxiröfFEN selbst gewusst (ISS\&, p. 3\$). Die Armform bleibt trotzdem immer noch das leicht verwertbarste Merkmal, und die eben erwähnten Fälle bedingen, wie noch zu erörtern sein wird, kein Schwanken in der Gruppierung. In einem l'unkt, kann ich VANHÖFFEN nicht folgen, nämlich in der Scheidung von triptera und trigona, wie oben 
bereits ausführlich erörtert wurden. Auch ist die einfache Nebeneinanderstellung von 7 Gruppen, obschon praktisch, doch nicht den natürlichen Verhältnissen entsprechend; einzelne der Gruppen sind untereinander viel näher verwandt als mit andern; das zeigt sich schon in der Armform selbst. Es sind darnach aber auch eine Reihe weiterer (in der Tabelle aufgefuhrter) Merkmale zu prifen, die sich eventuell mit den Unterscheidungen der Armform combinieren, zum Teil untereinder parallel gehen, und nach alle dem ein natürliches System zu versuchen.

A) Armform. Nachdem die HaEckel'schen Gattungen Archi-, Canno- und Haplorliza, die früher von VANröfFEN als simplicia zusammengefasst wurden, jetzt als „verstuimmelte Rhizostomeen", gewiss mit Recht von ihm gestrichen sind (1902), zeigen die dichotoma, mit einfach zweifligligen Armen, die ursprünglichste Verzweigungsform. Von ihr lassen sich die triptera, wie erwähnt, leicht ableiten, und davon auch alle Modificationen, die scapulata und lorifera, wie noch zu zeigen sein wird; nicht so leicht jedoch die Fiederform der pinnata. Mit dieser ist nicht die seitliche Fiederung zu verwechseln, wie sie bei dichotoma und auch triptera eintreten kann, nämlich dass ein Hauptast durch Selbstständigwerden der Krausenreihen, secundär seitliche Fiederäste entsendet - dabei bleibt die dichotome oder triptere Form immer noch erkennbar -, sondern der Unterarm selbst löst sich in mehrere unregelmässige Aeste auf (s. Cassiopcia), die sich weiter gabeln. Im Gegensatz zu den seitlich comprimirten Aesten der dichotoma, sind diese Aeste meist drehrund, tragen aber die Krausen doch nur auf der Unterseite. Ein Uebergang lässt sich nicht von Formen wie Cotylorhiza aus, sondern von Netrostoma aus construiren. Hier sind die Arme ebenfalls mehr drehrund, die ganze Armform gredrungener; dadurch werden die zwei Hauptäste im Verhältniss zu den seitlichen Fiederästchen kleiner und zuricktretend (Fig. 37); so dass von der Dichotomie von Cotylorhiza hier ein Uebergang zur Fiederung von Cassiopeia bewerkstelligt ist. Umgekehrt zeigt auch die Fiederung von Cassiopcia (z.B. bei C. cyclobalia laut Scuultze) eine Hinneigung zur Dichotomie, indem unter den unregelmässigen Seitenästen zwei Hauptäste stärker hervortreten.

Die Ableitung der dreiflugligen aus der zweiflugligen Form ist in der Litteratur so oft erörtert, dass ich hier nur die letzte Fassung von L. S. Scnultze angeben möchte: „aus den beiden Gabellappen am Distalende jedes der 8 Mundarme gehen unter Verschiebung ihrer Insertionsbasis die beiden Dorsalfligel mit den abaxialen Krausenreihen hervor". Hierin stimmen von einzelnen Modificationen abgesehen die Autoren bis zu DELAGE (Traité de zoologie) überein. Auf die für die Ableitung bedeutsamen Verhältnisse des Canalsystems in den tripteren Armen habe ich ebenfalls oben an mehreren Stellen (z.B., p. 57) hingewiesen. Die Tendenz, einen krausenfreien Endteil zu bilden, bedingt durch Aenderung im Krausenansatz, ist allen tripteren Formen und den davon ableitbaren gemeinsam. Der Endkolben von Rhizostoma ist dieselbe Bildung wie der von Mastigias und wohl auch wie der einiger lorifera; und alle diese lassen trotz Modificationen die dreifliiglige Grundform deutlich erkennen (s. Fig. 75, 90 und 93). Fast allen gemeinsam und schon von den dichotoma überkommen, ist auch die Tendenz an den Hauptiisten, resp. Hauptkrausenreihen, secundäre Fiederäste auszubilden (z.B. Crossostoma, Ly'chorhiza). Diese Seitenäste können grössere Selbstständigkeit gewinnen und auseinanderriicken, z. 13. bei Mastigias; besonders thuen dies die obersten Aestchen, und hieraus leiten sich die Schulter- 
krausen der scapulata ab. Die Verwachsung der Oberarme ist ebenfalls kein vereinzeltes Vor. kommen, sondern erscheint ausser bei den scapulata (Stomolophus) auch bei den lorifera (Himantostoma u. a.). Eine andere Weiterbildung kann die ursprünglich dreifluglige Form einschlagen, indem sich der Stamm mächtiger entwickelt, die Flügel und ihre seitlichen Krausen etwas zurückbleiben. Dann wird, namentlich am distalen Ende, der Einschnitt zwischen den Flügeln immer seichter; die Krausen sind hier sitzend und greifen auf die Flächen über, so dass allmählich am Distalende eine dreikantige Form entsteht mit scheinbar allseitigem Krausenbesatz, wie bei Crambessa. Wenn sich diese Form durch einseitiges Wachstum sehr verlängert, so entsteht die "Riemenform" der Arme; doch ist dieser Uebergang nur ganz allmählich; junge Exemplare von loriferen zeigen noch verhältnismässig kurze Arme. Auch im Alter haben nicht alle loriferen so extrem verlängerte Arme wie z.B. Thysanostoma, und selbst die extremsten zeigen an vielen Strecken der Arme noch deutlich die Flügelform und am Proximalende seitliche Fiederästchen. Auch krausenfreie Endkolben sind möglich, so dass also die Gruppe der loriferen keine derart scharf abgrenzbare ist, dass nicht ihre Züge auch bei andern dreiflügligen i. w. S. resp. Multicrispen wiederkehrten. Die Weitereinteilung der Multicrispen wird noch nach andern Gesichtspunkten als der Armform zu erfolgen haben; sie als ganzes den "Unicrispen", also den pinnata + dichotoma, gegenüber zu stellen, ist auch darum nicht angängig, weil sie zu der einer Gruppe, den dichotoma, viel nähere Beziehungen haben und Uebergänge zeigen, während die pinnata mehr abseits stehen.

B) Genitalostien resp. Atemhöhlen. Die Gestalt und Grösse der Ostien, die in den Subgenitalraum füren unc die auch als interradiale Atem- oder Trichterhöhlen bezeichnet werden, ist im Gegensatz zum Subgenitalraum selbst, als Merkmal wohl verwendbar und wird von dessen ontogenetischen Veränderungen nicht betroffen. Die Gruppe der pinnata zeigt 4 sehr enge kreisrunde Oeffnungen, die zu 4 getrennten Höhlungen führen; die dichotoma (Cephea, Cotylorhiza, Netrostoma) haben kaum grössere, ungefähr trichterförmige Oeffnungen; der innere Querschnitt und die Verhältnisse der Höhlen, zu denen sie sich erweitern, sind verschieden. Bei allen stellen sie aber nur verschwindend kleine Löcher an der massiven Armscheibe dar. Bei den tripteren resp. multicrispen sind sie, auch im mindest ausgebildeten Fall, viel weiter, so dass man von Ostien und zwischenliegenden Pfeilern reden kann, deren gegenseitiges Breiteverhältniss varïrt. Bei einer Anzahl von Formen sind sie schmäler wie die Pfeiler, schlitzförmig; werden öfters noch durch Klappen eingeengt. Hierher gehören Toxoclytus, Loborhiza, Crambione, Rhizostoma u. A. Die Verhältnisse des Porticus selbst variiren hier zwischen vollkommener Trennung, unvollständiger und vollständiger Vereinigung. Bei anderer Formen sind die Ostien vielmals breiter wie die Pfeiler; der einheitliche Subgenitalsaal erscheint dann als ein Teil der Subumbrella, die Pfeiler als blosse Unterbrechungen. Hierher gehören z.B. Crossostoma, Thy'sanostoma u. A.

C) Das periphere Canalsystem. Die Verzweigung der vom Magen aus in die Subumbrella gehenden Canäle ist mannigfacher als man denken sollte und zeigt schon innerhalb der triptera resp. multicrispa grosse Verschiedenheiten, noch grössere wenn man auch die dichotoma und pinnata dazu nimmt. Die einfachsten Verhältnisse zeigen auch hierin die gewöhnlichen zweiflügligen Formen. Vom Magen aus gehen, (s. Fig. 43 und $43 a$ von Netrostoma) den Sinnes- 
organen entsprechend, 8 rhopalare Canïle aus, dazwischen mehr oder weniger (mindestens 3) weitere Radialcanäle, deren und Zahl Caliber gegenüber den 8 rhopalaren bei den einzelnen Gattungen verschieden ist. Alle zeigen einen ziemlich gestreckten Verlauf und anastomosieren durch Quercanäle. Es entsteht so ein nach der Peripherie zu immer enger werdendes Netz, das keinen Ringcanal erkennen lässt.

Anders die pinnata. Hier ist zwar ebenfalls ein Netzwerk mit radiärer Tendenz vorhanden, in welchem die rhopalaren Canäle ( 16 und mehr) und einige Zwischencanäle durch Verlauf und Caliber hervortreten (s. Haeckel I879, Taf. 37, Fig. 2). Dann aber folgt nach allmählicher Verengerung des Netzes ein innerer Ringcanal; jenseits desselben ein zuerst weiteres, dann sehr verengertes Maschenwerk und hierauf ein zweiter, abschliessender Ringcanal. Hierin stehen ebenfalls die pinnata unter allen Rhizostomen vereinzelt da, während sich die verschiedenartigen Verhältnisse innerhalb der triptera und übrigen multicrispen von einander ableiten lassen. Ein äusserer Ringcanal ist bei letzteren niemals zu erkennen, ein innerer meist vorhanden, kann jedoch (secundär verwischt:) ebenfalls fehlen. Man kann ferner unterscheiden, ob von der Magenperipherie ausser den 8 rhopalaren Canälen noch zahlreiche Radialcanäle abgehen, oder nur einer zwischen je zwei Rhopalarcanälen. Im ersteren Fall ist schon diesseits des Ringcanals ein Anastomennetz vorhanden, im letzteren Fall bleiben zwischen den $8+8$ Radiärcanälen freie Felder übrig, die durch blindendende Canalverzweigungen vom Ringcanal aus nur unvollkommen ausgefullt werden. Bei Rhizostoma, Rhopilema, Stomolophus gehen die $8+8$ Canäle sämmtlich bis zum Schirmrand, der Ringcanal ist verwischt; von seiner ungefähren Stelle ist peripher ein engmaschiges, centralwärts ein blindendendes, hoch hinaufsteigendes Anastomosennetz zu erkennen (s. Taf. V, Fig. 2 VAnhöffex 1888, Claus is78, Fig. 42). Die scapulata verhalten sich also hierin untereinander einheitlich und von den übrigen multicrispen abweichend. Die anderen Formen mit $8+8$ Radiärcanälen unter den tripteren, lassen nur die 8 rhopalaren Canäle bis zur Peripherie gehen, die übrigen 8 enden schon im inneren Ringcanal (s. hier Fig. 48). Hierher gehören u. A. Pseudorhiza, Lychnorhiza, Crambione, Crambessa und vielleicht auch einige Himantostomiden (lorifera). Bei allen ist ein äusseres engmaschiges Gefäss peripher vom Ringcanal in gleicher Weise entwickelt, die inneren blinden Canäle sind jedoch bei den einzelnen Gattungen in sehr verschiedener Weise ausgeprägt. Bei Pseudorhiza sind io blinde, gerade und parallel verlaufende Canäle zwischen den radialen und adradialen vorhanden (s. Fig. I 4 bei LENDENFELD 1888); bei Lychnorhiza wenige auffallend breite und schräg gestellte Canäle die zuweilen zu einem Gefässbogen verschmelzen, (HAECKel, I879, Taf. 34, Fig. 2, VANhöffen, I888, Taf. III, Fig. 2); bei Crambione sind zahlreiche engere Canäle vorhanden, die unter cinander ein Netzwerk bilden (hier Fig. 48 ent), und bei Crambessa endlich ist dieses Netzwerk noch weiter ausgebildet und nicht nur mit dem Ringcanal, sondern auch mit den Radiärcanälen in Zusammenhang (Grexacher und Nolr, Fig. I3), ähnlich wie bei Rhizostoma.

Bei den übrigen tripteren Formen sind zwischen je zwei Rhopalarcanälen sehr zahlreiche (7 und mehr) Radiärcanäle schon vom Magen aus zu erkennen, so dass das innere Anastomosennetz nicht blind endigt, sondern mit dem Magen selbst zusammenhängt (s. hier Fig. $5^{8}$ und VANHÖFFEx I 888, Taf. IV, Fig. 7). Das periphere Netz ist wie überall sehr engmaschig. Bei einigen Formen ist der Ringcanal sehr deutlich, z.B. Mastigias, Desmostoma, Phyllor/iza; 
bei anderen verwischt z.B. Loborhiza, Crossostoma, und einigen Himantostomiden (lorifera). Diese letztere Gruppe ist also in Bezug auf des Canalsystem nicht ganz einheitlich, sondern wie es auch bei der Armform schien, von verschiedenen Gruppen der tripteren ableitbar; die scapulata dagegen sind hierin eine geschlossene Gruppe. Die triptera selbst sind noch weiter einzuteilen; die betr. Gruppen entsprächen aber nicht den VANHöfrex'schen triptera und trigona, eher noch den CLaus'schen Familien der Lychnorhizidae und Catostylidae (partim). Die ersterwähnten Gruppen der pinnata (Cassiopeidae Claus) und dichotoma (Cepheidae Claus) haben auch hier im Canalsystem entsprechende Unterschiede.

C) Die Subumbrellarmuskulatur. Die Verhälnisse der Muskulatur sind weniger mannigfach, aber in den Hauptgruppen um so schärfer verschieden. Auszugehen hat man auch hier von den dichotoma. Sie zeigen die in einer Subumbrella möglichen Systeme der Zugrichtung, das radiäre und das circuläre, beide entwickelt, die circuläre Lage jedoch nur sehr schwach, die radiäre sehr stark, so dass die Subumbrella von mehr oder minder dicht liegenden radiären Muskelstreifen durchzogen wird. Die circulären Fasern sind nur am Rand und in der Umgebung der Armscheibe deutlich.

Ganz anders die pinnata. Hier sind die Muskeln in den auffälligen, sonst nirgends vorkommenden Fiederarcaden angeordnet, entsprechend der Rhopalienzahl (Fig. 30). Abzuleiten ist diese Anordnung jedenfalls aus der circulären durch entsprechende Einziehungen infolge radiärer Ziige. In der That biegen sowohl nach der Armscheibe wie nach der Peripherie zu die Arcadenzüge in gewöhnliche Circulärmuskulatur um, Schultze 1898, Fig. 4) wie ich auch bei einer fossilen Form nachweisen konnte (I902, Taf. XXII, Fig. I und Taf. XXIII, Fig. 3). Ferner sind beim recenten wie beim fossilen noch radiäre Züge, aber nicht überall, sondern nur in bevorzugten Radien zu erkennen.

Bei allen übrigen Formen der Rhizostomen treten die Radiärzüge bis zu völligem Verschwinden zurück; die triptera, wie die lorifera und die scapulata zeigen nur eine Circulärmuskulatur; es bilden also die multicrispa auch hierin eine sehr einheitliche Gruppe. Die möglichen Unterschiede beziehen sich nur darauf, dass bei den einen Formen, entsprechend den weit offenen Ostien, die Muskulatur in den Interradien weiter centralwärts reicht und durch stärkere Ausbildung einen besonderen peripheren Ring erkennen lässt, ferner darauf, ob dieser Ring in den Hauptradien unterbrochen wird oder einheitlich ist. Bei Rhizostoma etc., also den scapulata, sind I 6 Unterbrechungen vorhanden, es entstehen dadurch 16 radiäre Muskelfelder mit der Spitze weit nach innen reichend, bei llastigias u. A. sind 8 Unterbrechungen u. dem zufolge 8 getrennte Muskelbogen vorhanden; bei anderen z.B. Crossostoma, ist der äussere Muskelring einheitlich, die innere Circulärmuskulatur zerfällt durch die Armpfeiler etc. in Felder. Hieran schliefsen sich auch die lorifera.

D) Die Rhopalien, Ein letztes und bisher noch wenig gewuirdigtes Merlimal bilden die Sinneskolben. Sie sind zwar alle nach einem und demselben Grundtypus gebaut, der auch für die Semaeostomen zutrifft und von dem der Ephyropsiden etc. abweicht; zeigren aber im einzelnen doch eine Reihe von Verschiedenheiten, die zur Kennzeichnung verschiedener Rhizostomeengruppen mithelfen können.

Am vollkommenen Rhopalium ist zu unterscheiden: a) der Otolithensack selbst mit 
entodermaler Axe, bulböser Auftreibung proximal, den Concrementen distal. b) ein dorsales, iiber dem Concrementteil gelegenes, und ein ventral, am Bulbus gelegenes Nerven-Sinnesepithel. c) ein eventuelles Auge am dorsalen Epithel des Kolbens. d) eine äussere, in die Schirmgallerte eingesenkte Sinnesgrube, die durch radiäre Falten noch weiter differenziert sein kann.

Die einfachsten Verhältnisse zeigen auch hierin die dichotoma. Der Kolben selbst ist gestreckt und tentakelähnlich; am dorsalen Sinnesepithel zeigt sich keinerlei Augendifferenzierung. Die äussere Sinnesgrube fehlt ganz. Dies ist der Fall bei Cotylorhiza (Hesse), Cephca, Netrostoma (s. 0. p. 37, Fig. 97 und I03). Bei den pinnata kommt noch ein kleiner Pigmentfleck am distalen Ende des Concrementsacks hinzu; ferner ist eine äussere Sinnesgrube, klein und ohne Radiärstreifung erkennbar (Keller i $88_{3}$, Bigelow i goo, MAAs s. o. p. 45 und Fig. 98).

Bei den tripteren und davon abzuleitenden Formen ist diese äussere Sinnesgrube sehr verschieden entwickelt, manchmal nur eine kleine und seichte sichelförmige Grube (Pscudor/iza, Mastigias) (Fig. 99), manchmal gross und muschelförmig, mit tiefen Rinnen und Falten, die eine drüsenartige Differenzierung des Epithels ausser den Sinneszellen enthalten; hierzu zählen Crambione (Fig. IOO), Crambessa, die lorifera (Fig. 10I) und scapulata. Letztere zeigen keinen Pigmentfleck am Epithel des Concrementsacks; die triptera sind in letzterer Hinsicht sehr verschieden; manche Gattungen haben einen unpaaren (Fig. 99), manche einen paarigen (Fig. I00), manche gar keinen Ocellus (s. Tabelle). Gewisse Unterschiede treffen also für die grösseren Gruppen zu, andere sind nur als Gattungsmerkmale zu brauchen; das letztere gilt auch für die Armanhänge, Kolbenblasen sowohl wie Peitschenfilamente, die in der Tabelle noch berücksichtigt sind.

Aus alledem geht hervor, dass wir unter den Rhizostomeen drei natürliche grössere Gruppen machen können. Die beiden ersten lassen sich auch nach der Armform bezeichnen, als dichotoma und pinnata, die dritte enthält verschiedene Armformen, triptera, scapulata und lorifera, die aber alle auf die dreiflighlige Form zu beziehen sind. Die beiden ersten Gruppen zusammen als Unicrispa den andern, den Multicrispa, gegenüber zustellen, ist nicht richtig, weil bei allen Merkmalen ersichtlich ist, dass die dichotoma allein den multicrispa näher verwandt sind, und die pinnata viel weiter abseits stehen, auch von den dichotoma. Die verschiedenen Gruppen der Multicrispa den dichotoma und pinnata zu coordinieren ist ebenfalls nicht ganz natuirlich, da sie untereinander viel näher verwandt sind und bald in diesem, bald in jenem Merkmal gruppenweise ibereinstimmen. Am besten gesondert und untereinander übereinstimmend erscheinen noch die scapulata, unter den triptera sind zwei, vielleicht sogar drei Gruppen zu machen (nicht trigona abzugrenzen), und die lorifera sind von verschiedenen tripteren Gruppen polyphyletisch abzuleiten.

Der Stand unserer Kenntnis lässt sich etwa in folgendem Schema ausdrücken:

\section{Rhizostomata.}

I. Arcadomyaria. Muskulatur in Fiederarcaden angeordnet. Mundarme unregelmässig gefiedert („pinnata" Vanhöffen). Canalsystem mit doppeltem Ringcanal, i 6 Radiärcanälen und weiteren Zwischencanälen, die vom Magen ausgehend ein inneres und äusseres Anastomosennetz bilden. Subgenitalostien kreisrund, eng, in 4 getrennte Höhlen führend. Rhopalien ohne Auge und ohne äussere Sinnesgrube.

Fam. Cassiopeidae. Gen. Cassiopeia, Torcuma. 
II. Radiomyaria. Muskulatur in starken Radiärzügen angeordnet; circuläre Züge sehr schwach. Arme zweigabelig ( dichotoma” Vanh.). \& Radiärcanäle und (3-x) \& Zwischencanäle vom Magen ausgehend, die ein peripher allmählich enger werdendes Maschenwerk bilden, ohne Ringcanal. Ostien eng, trichterförmig; Subgenitalporticus verschieden, bald getrennte Höhlen, bald Vereinigung bildend. Rhopalien mit Pigmentfleck und kleiner äusserer Sinnesgrube.

Fam. Ce pheidae. Gen. Cephea, Cotylorhiza, Netrostoma.

III. Cycloniyaria. Muskulatur ringförmig angeordnet. Armform dreifluglig oder davon ableitbar ( triptera + trigona, scapulata, lorifera” Vanh). Ostien schlitzförmig oder sehr weit.

A. I6 zum Schirmrand gehende Radiärcanäle, dazwischen blind endigendes Anastomosennetz. Arme mit Schulterkrausen („scapulata” Vanh.). Genitalostien schlitzförmig, durch Klappen geteilt. Rhopalien ohne Ocellus, mit äusserer radiärgefalteter Grube.

Fam. Rhizostomidae. Gen. Rhizostoma, Rhopilema, Stomolophus.

(Rhizostomidae + Stomolophidae) Claus.

B. 8 zum Schirmrand und $\delta$ zum Ringcanal gehende Canäle, aussen enges Netz, innen wenige blinde Maschen.

I. Arme dreiflüglig, meist mit seitlichen Fiederästen. Ostien schlitzförmig. Rhopalien mit Pigmentfleck und äusserer Grube mit Radiärfalten.

Fam. L y chnorhizidae. Gen. Lychnorhiza, Crambione, Crambessa.

2. Arme stark verlängert, noch dreiflüglig. Ostien weit klaffend. Rhopalien s.o.

Fam. Le ptobrachidae (part.)

C. 8 zum Schirmrand und dazwischen zahlreiche nur zum Ringcanal gehende Canäle; aussen enges, innen weiteres Maschennetz bildend.

I. Arme stark verlängert, riemenförmig. Ostien weite Querschlitte.

Fam. Leptobrachidae. Gen. Thysanostoma, Leptobrachic, Himantostoma.

2. Arme dreiflüglig, nicht verlängert. Ostien weit. Rhopalien mit Pigmentfleck und kleinerer äusserer Grube ohne Radiärfalten.

Fam. Catostylidae. Gen. Loborhiza, Crossostoma, Mastigias, Desmostoma.

Da durch die verschiedenen Conservierungsweisen bald die einen, bald die anderen Organisationsverhältnisse mehr hervortreten, z.B. bei Chromsäuregemischen die Muskulatur, während das Canalsystem oft schwer sichtbar ist, umgekehrt bei Formol das Canalsystem sehr deutlich durchschimmert und, die Muskulatur zurücktritt, so empfiehlt es sich schon aus praktischen Gründen, nicht nur im Interesse eines natürlichen Systems, mehrere Merkmale bei der Bestimmung zu berücksichtigen.

In der oben stehenden analytischen Uebersicht sind nicht alle bekannten und in der Tabelle p. So angeführten Gattungen in die Familien eingereiht, z. T. weil die Zugehörigkeit bei einigen noch fraglich ist, z. T. weil sich die Aufstellung einer weiteren Familie unter den tripteren als notwendig erweisen wird. Weiteres Material wird wohl jetzt den endgiltigen Ausbau des Rhizostomeen-Systems ermöglichen. 


\section{Verzeichnis der citierten Litteratur.}

I865 AgAssiz, A. North-American Acalephae. Illustrated Catalogue of the Museum of Comp. Zool. Cambridge Mass.

ISgS — - and MAyer, A. G. On some Medusae from Australia. Bull. Mus. Comp. Zool. vol. XXXII, No 2. I899 - - Acalephs from the Fiji-Islands. ibid. vol. XXXII, $N^{n} 9$.

1902 - Reports on the scient. Results of the Exp. to the Tropical Pacific.... I899/1900, III. Medusae. Mem. Mus. Comp. Zool. vol. XXVI.

I 860 Agassiz, L. Contributions to the Natural History of the United States. vol. III.

I 862 — ibid. vol. IV.

I 892 Bigelow, R. P. On a new species of Cassiopeia from Jamaica. Zool. Anz. I5. Jahrg.

1900 - - The Anatomy und Development of Cassiopeia xamachana. Mem. Bost. Soc. Nat. Hist. vol. 5.

I 38 BRANDT, J. Fr. Ausfuhrliche Bescheibung der von C. H. Mertens auf seiner Weltumsegelung beobachteten Schirmquallen. Mém. Acad. St. Pétersb. 6 sér. T. 4. sc. nat. T. 2.

I\$23 DELLE ChIAje. Memorie sulla storia degli animali senza vertebre. Napoli.

I896 Chun, C. Beiträge zur Kenntnis Ostafrikanischer Medusen und Siphonophoren. Mitteil. Naturh. Museum. Hamburg. XIII. Bd.

I.7. Claus, C. Studien über Polypen und Quallen der Adria. I. Acalephen. Denkschr. Naturw. Math. Classe Kais. Ak. IViss. Bd. 38. WVien.

I $88_{3}$ - Untersuchungen über Organisation und Entwicklung der Medusen. Prag und Leipzig.

I8S4 - Die Ephyren von Cotylorhiza und Rhizostoma etc. Arb. Zool. Inst. Wien. V. Bd.

IS88 —- Die Classification der Medusen mit Rücksicht auf die sog. Peromedusen. ibid. VII. Bd.

I891 — Über die Entwicklung der Scyphostoma von Cotylorhiza, Aurelia I. Ibid. IX. Bd.

1892 - Über die Entwicklung der Scyphostoma von Cotylorhiza etc. II. sowie uber die systemat. Stellung der Scyphomedusen. Ibid. X Bd.

IS 3 ; Ehrenberg, C. G. Über die Acalephen des roten Meeres und den Organismus der Medusen der Ostsee. Abh. Berl. Akad. WVissensch.

1,-y EMER, TH. Die Medusen, physiologisch und morphologisch auf ihr Nervensystem untersucht. Tübingen.

I'n, Eschisciroltz, FR. System der Akalephen. Berlin.

I: 2 Fewkes, J. IV. Studies of the Jelly fishes of Narragansett Bay. Bull. Mus. Comp. Zool. vol. S.

I8S2 - Reports on the Acalephs of the Blake. ibid. vol.9.

ISi - Report on the Medusae collected in the Gulf Stream by the Albatross $83 / 84$. U. S. Fish. Comm. XiI. Rep.

IS. - Report... 85/86. Ibid. XIV. Rep.

IS: - Are there deep sea Medusae? Ann. und Mag. Nat. Hist. ser. 6. vol. I.

isi GoetTe, A. Verzeichnis der Medusen, welche von Dr. Sander, Stabsarzt auf S. M. S. Prinz Adalbert gesammelt wurden. Sitzungsber. Berl. Akad. XXXIX bd.

I,$\cdots$, Grenacher, H. und Noll, F. C. Beiträge zur Anatomie und Systematik der Rhizostomeen. Abh. Senckenb. Naturf. Gesell. Frkft. Bd. X. 
1857 Gegenbaur, C. Versuch eines Systems der Medusen; mit Beschreibung neuer und wenig gekannter Formen. Zeitschr. fo wiss. Zool. Bd. VIII.

I887 HaACKE, W. Die Scyphomedusen des St. Vincent Golfs. Jena, Zeitschr. f. Naturw. Bd. XX.

I879 Haeckel, E. Das System der Medusen; mit Atlas. Jena.

I88 I _- The deep sea Medusae. Chall. Rep. Zool. IV.

188 I Hamann, O. Die Mundarme der Rhizostomen und ihre Anhangsorgane. Jenasch. Zeitschr. f. Naturw. Bd. I 5 .

1878 Hertivig, O. und R. Das Nervensystem und die Sinnesorgane der Medusen. Leipzig.

I895 Hesse, R. Untersuchungen über das Nervensystem und die Sinnesorgane der Medusen. Zeitschr. f. wissensch. Zool. Bd. 60 .

I849 Huxley, T. H. On the Anatomy and Affinities of the Family of the Medusae. Phil. Trans. Roy. Soc. London.

1901 Kassianow, N. Studien über das Nervensystem der Lucernariden etc. Zeitschr. f. wiss. Zool. 69. Bd.

I86r Keferstein, W. und Ehlers, E. Zoologische Beiträge Leipzig.

I883 Keller, K. Untersuchungen über neue Medusen aus dem roten Meer. Zeitschr. fo wissensch. Zool. Bd. 38 .

I $895 \%$. Kishinouye, K. On Thysanostoma denscripsum nov. spec. Zool. Mag. Tokyo. vol. VII.

I 895 3. - - Description of a new Rhizostoma, Mastigias physophora. ibid.

I 899 - Edible Medusae (Rhopilema esculenta und verrucosa n. sp.) Zool. Jahrb. Abt. für System. etc. XII. Bd.

1853 KöLLIKER, G. Bericht über einige im Herbst I852 in Messina angest. Unters. II. Über Quallen. Zeitschr. f. wiss. Zool. Bd. IV.

I882 Lendenfeld, R. von. Über Coelenteraten der Südsee. II. Mitt. Cyanea Annaskala. Zeitschr. f. wiss. Zool. Bd. 37 .

IS8S —- VII. Die australischen rhizostomen Medusen. ibid. Bd. 47

I830 Lesson, R. P. Centurie Zoologique. Paris.

I897 MAAS, O. Die Medusen, in: Reports on an Exploration..... by the U. S. Steamer Albatross etc. Mem. Mus. Comp. Zool. vol. XXIII.

I899 - - Sur la distribution geographique et bathymetrique des Méduses provenant des campagnes scientifiques du Prince de Monaco. Bull. Soc. Zool. de France. Paris.

1902 - Über Medusen aus dem Solenhofer Schiefer und der unteren Kreide der Karpathen. Palaeontographica. Bd. 48. Stuttgart.

I8 io PÉron, F. und Lesueur, C. A. Tableau des caractères génériques et specifiques de toutes les espèces de Méduses connues jusqu’à ce jour. Ann. Mus. Hist. nat. Paris. vol. 14.

I833 Quoy et Gaimard. Voyage de l'Astrolabe. Zool. IV. Zooph. Paris.

I889 Scheiviakoff, W. Beiträge zur Kenntnis des Akalephenauges. Norph. Jahrb. Bd. X.

I 897 Schultze, L. S. Rhizostomen von Ternate. Abh. Senckenb. Naturf. Gesellsch. Frankfurt. Bd. XXIV.

1898 - - Rhizostomen von Ambon. Denkschr. der Math. Nat. Gesellsch. Jena (in: Semon, Forschungsreisen).

183I Tilesius, W. G. Beiträge zur Naturgeschichte der Medusen. I. Cassiopeiac. Nova Acta Acad. Nat. Cur. XV. 2 suppl. I 829. Breslau und Bonn.

1889 VANhöfFen, E. Untersuchungen über semaeostome und rhizostome Medusen. Bibl. Zoolog. Cassel. Bil. I, lieft 3 .

1892 — Die Akalephen der Plankton Expedition. Ergeb. Plankt. Exp. II. k. Kiel und Leipzig.

1900 - Über Tiefseemedusen und ihre Sinnesorgane. Zoolog. Anz. 23 Jahrg.

1902 - Die acraspeden Medusen der deutschen Tiefseeexpedition 98/99. Ergeb. Deutsche Tiefsee Exp. Bd. III. Jena. 



\section{TAFEL I.}

Iijg. I. Linerges draco Hckl. von der Subumbrellarseite aus, junges Exemplar, etwa 8 mal vergrössert; in natürlichen Farben. Man sieht die Anlage der 8, paarweise in den Perradien genäherten Gonaden, sowie die 16 , in einer circulären Reihe liegenden Subumbrellarbläschen (sb). Der Schirmrand ist etwas nach innen gebogen, so dass die Verlängerungen der Pedalfurchen in der Mitte eines jeden Lappens sichtbar sind.

Fị. 2. Linerges draco Hckl. Ein Stuck Schirmrand von innen, stärker vergrössert. Die Lappenspange (lo s) geht nicht bis zum Distalende; dadurch kommt eine Art Ringcanal (ri) zu Stande. Dieser zeigt zahlreiche periphere, verzweigte Ausläufer (ri!). Am Rand des Lappen erscheinen die exumbrellaren Nesselwarzen (nw).

Iïg. 3. Atolla Valdiviae. Vanh. Magengrund eines Exemplars mit abgerissenem Rohr, um die weit vorspringenden interradialen Cathammen mit den Gastralfilamenten zu zeigen: perradial 4 enge Oeffnungen.

I.ig. +. Atolla Valdiviae Vanh. Kleines perradiales Stiick aus der Subumbrella, mit dem Magenansatz, dem Gaumenknoten, einem Paar Gonadenanlagen und den sog. Excretionsorganen (exc) zu beiden Seiten des perradialen Magenansatzes.

l'is. 5. Nausithoe picta A. Ag. und Mayer. Stuick der Subumbrella in starker Vergrösserung; mit Randlappen (lo), Lappenspangen (lo s) und Ringcanal (ri). Mit Circulärmuskulatur und ausserdem radiären Ziigen (mu ra) an der Tentakelbasis und den Subumbrellarplatten (su pl) uiber dem Muskelring. Mit ausgebogenen Gonadenschildern (gon), zu je zweien im Interradius, nahe den Gastralfilamenten (fil).

I'ig. 6-8. Nausithoe picta A. Ag. und Mayer. Gonadenlage bei verschiedenen, gleich grossen und geschlechtsreifen Exemplaren, und zwar Fig. 6 die 8 länglichen Schilder in gleichen Abständen, Fig. 7, einige genähert und ausgebogen, Fig. S. Alle S Schilder zu je zweien im Interradius einander zugebogen und genähert. (Perund Interradius sind an der Magenöffnung bezw. den Filamenten kenntlich).

Fig. 9. Aurclia aurita L. var. colpota Brdt. Stück der Subumbrella, um die regelmässige Verzweigung und den Abgang der Radiärcanäle zu zeigen, die ohne mit einander zu anastomisieren den Ringcanal erreichen. Naturl. Grösse und Farben. 

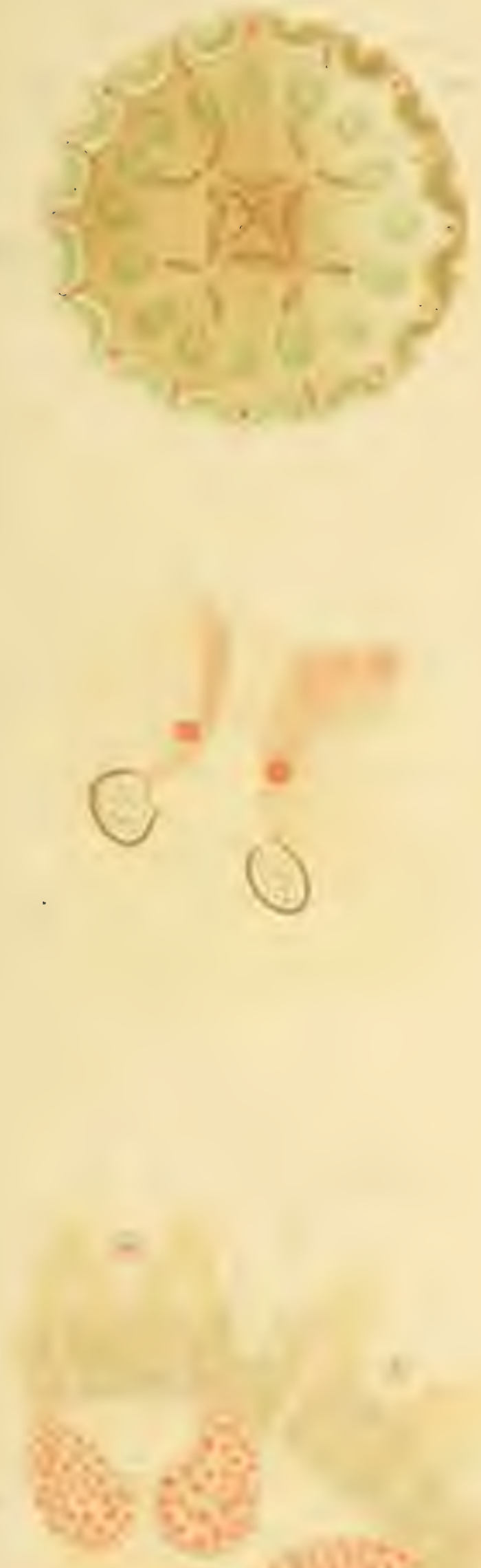

(). Mar, icl.

1
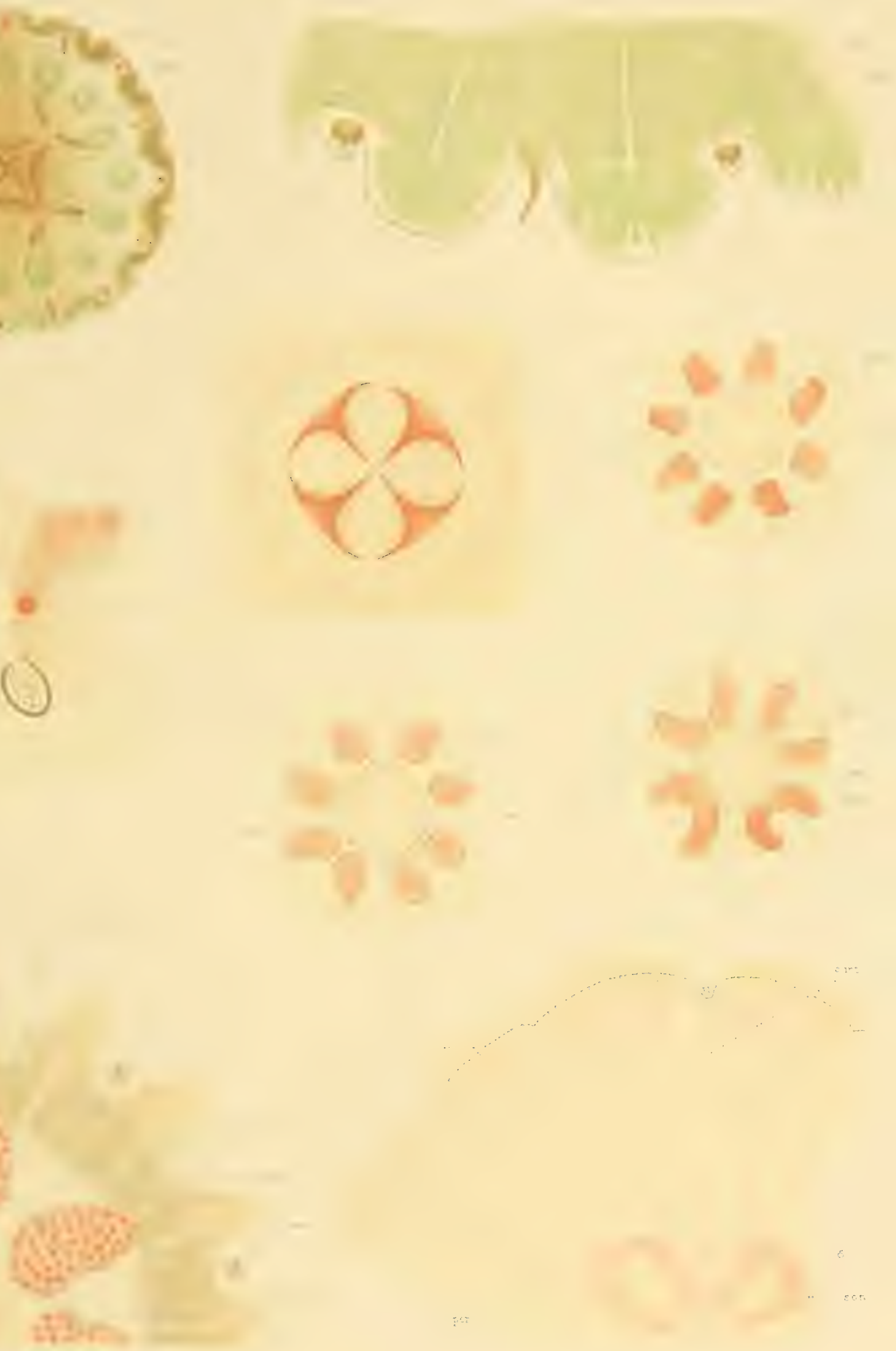




\section{TAFEL II.}

IFis. Io. Paraphyllina intermedia nov. gen. n. sp. von der Subumbrellarseite aus etwa, 8 mal vergrössert. Die 4 Rhopalien liegen genau in den Radien der Magenecken (Perradien), die 12 Tentakel in den Radien der Magenflächen (Interradien), also umgekehrt wie bei Periphylla. Die Gonaden zu je zwei an den Magenflächen. Muskulatur und Subumbrellarplatten wie bei Nausithoe. Ungefähr natürl. Farben. Magenecken weisslich, IIagenflächen mit rotem Streifen.

Fig. 1r. Paraplyyllina intermedia nov. gen. nov. sp. Ansicht eines andern Exemplars von der Seite. Radienverhältnisse wie oben. Magengrund mit den perradialen Oeffnungen und interradialen Filamentbiischeln durch die Exumbrella stark durchscheinend, schwächer das in die Subumbrella herabhängende Magenrohr. Circulärfurche (fos) und Pedalien (ped) der Exumbrella. Rhopalarpedalien schmäler als die tentakularen, Lappen (lo) gleich. Subumbrellarmuskulatur und Platten (su pl), letztere mit radiärer Streifung durchschimmerend; ferner die bräunlichen Gonaden.

Fig. I 2. Parapliyllina intermedia n. $g$; n. sp. Stück einer Subumbrellarplatte mit dem Tentakelansatz und radiärer sowie circulärer Muskulatur.

Fig. 1 ; : . . Parapluyllina intermedia n. g.; n. sp. Sinneskolben Fig. I 3 von aussen, schräg gesehen und Fig. I 4 genau im optischen Schnitt nach einem mikroskop. gefärbten Präparat. Otocyste (ot) unter der Deckschuppe (sq) geborgen, Auge mit Pigment (pi) und Linse (li) Sinnesepithelien (ect!) am Otocysten und am Bulbus. (Vgl. Fig. Io6, auf Taf. XI).

Iin. i 5. Pcriphylla dodecabostrycha Brdt. Rhopalium von innen, stark vergrössert. An die Deckschuppe (sq) angeschmiegt der Endkolben (ot) mit den Concrementen; weiter proximal der Bulbus, rechts und links nach dorsal übergreifend mit ectodermalem Sinnesepitheil (ect!) und entodermalem Pigment (ent. pi). 

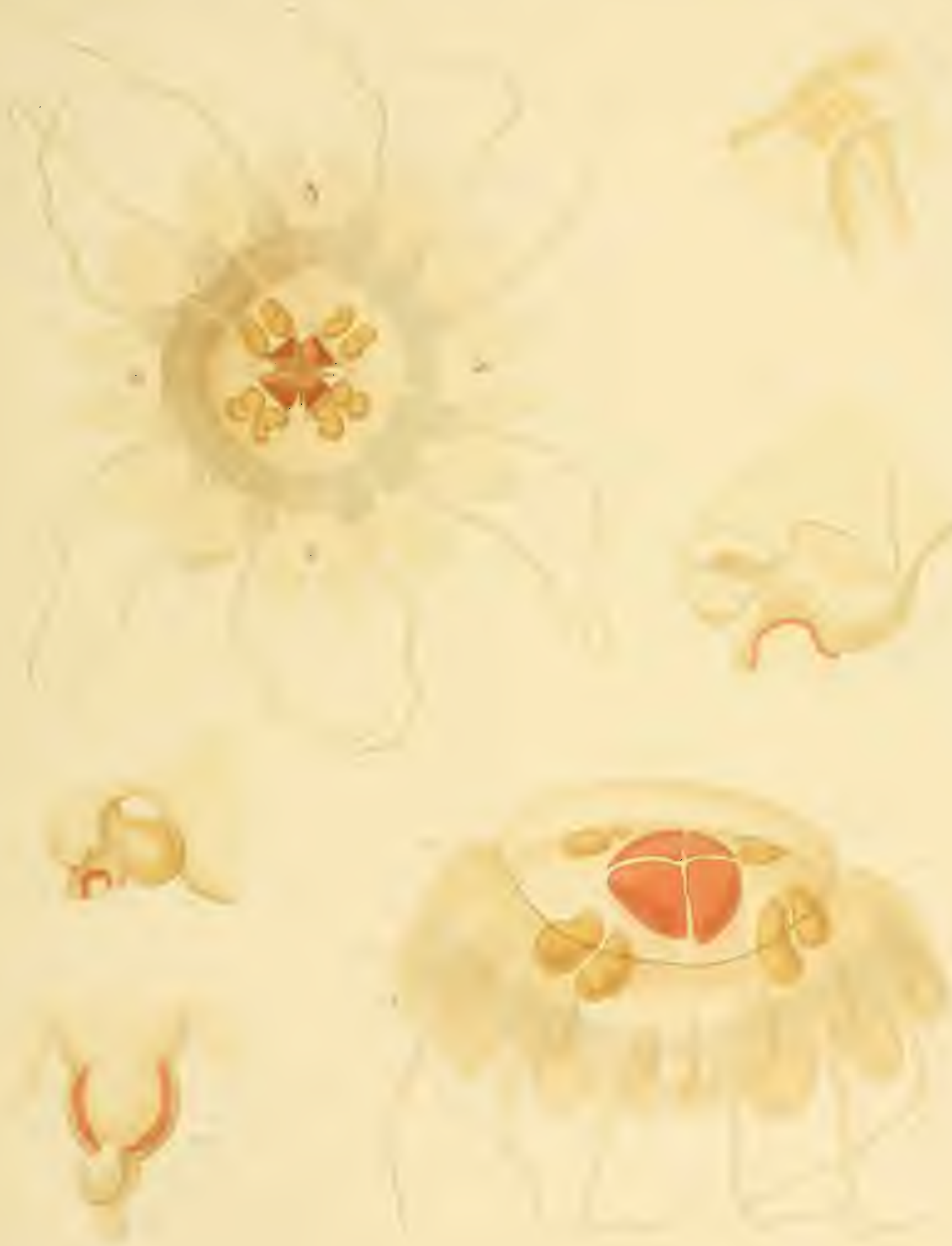

ก. Mras del.

Cirromolith. P. W. M. Trap. Leiden. 

. 


\section{TAFEL III.}

Iiv. I6. Atorella subglobosa Vanh. Von der Exumbrella aus etwa $10 \times$ vergrössert. 6 Tentakel mit 6 Rhopalien am Schirmrand alternierend. Deutliche Kranzfurche (fos) und Lappeneinschnitte. Nur 4 unpaare (einzelne) Gonaden von unregelmässiger Form, aber genauer Verteilung in den Interradien, die durch die Filamentbiischel gekennzeichnet werden.

I.in. 1. Atorella subglobosa Vanh. In der Subumbrella-Gallerte aufsteigende Tentakelwurzel, starke Vergr. Der Tentacularcanal bildet einen blinden, nicht hohlen Sporn, der auch die Abgangsstelle ungreift. Die Entodermzellen sind im Tentakel zunächst um einen Hohlraum (und mit regelmässig gestellten Kernen) geordnet; dann bilden sie eine solide Axe.

Iiig. 1. Atorella subglobosa Vanh. Das gedrungene Rhopalium von innen gesehen, Endkolben mit Concrementen in der Deckschuppe liegend; ventraler Bulbus mit hohem Ectoderm und dunklem Entoderm.

Fig. 19. Pericolpa campana Hckl. Seitliche Ansicht. Von den 4 Rhopalien 2 sichtbar. Kranzfurche (fos) trennt die gewölbte Schirmkuppel von dem etwas nach innen geschlagenen Lappenkranz. Die Pedalien erscheinen noch einmal zweigeteilt. Muskulatur (m cir), Gastralfilamente und die unregelmässig gestellten Gonaden durch die Schirmgallerte durchschimmernd.

Fir:. 2(1). Pericolpa campana Hckl. Ansicht von unten, mit eingeschlagenem Schirmrand. 4 Rhopalien und (anormal) 5 Tentakeln sichtbar, $(\mathrm{t} !)=$ überzählige. Pedalfurchen (pedf) markieren sich an der Peripherie.

Fig. 21, 22. Pcricolpa campana Hckl. Rhopalien von aussen und innen. Von aussen deckt die Schuppe vollständig den Endkolben; von innen ist er sammt den Concrementen deutlich sichtbar; ausserdem der Bulbus mit dem hohen Ectoderm und gefärbten Entoderm.

Fi:. 23. Atolla Valdiviac Vanh. Peripheres Canalsystem, durch Abschneiden des starken Ringmuskels und der Tentakelmuskeln sichtbar gemacht. Vom Ringsinus gehen zu jedem Rhopalium ein schmaler, zu jedem Tentakel ein breiter Radiärcanal ab. Letzterer ist schon an der Basis durch Cathammen in eine Schleife geteilt, deren mittlerer Ast an der soliden Tentakelaxe endigt, während die seitlichen sich je in die angrenzenden Lappen begeben und dort mit den entsprechenden, vom Rhopalarcanal, jedoch erst ganz distal entspringenden Canälen zu einem Festoncanal verbinden. 

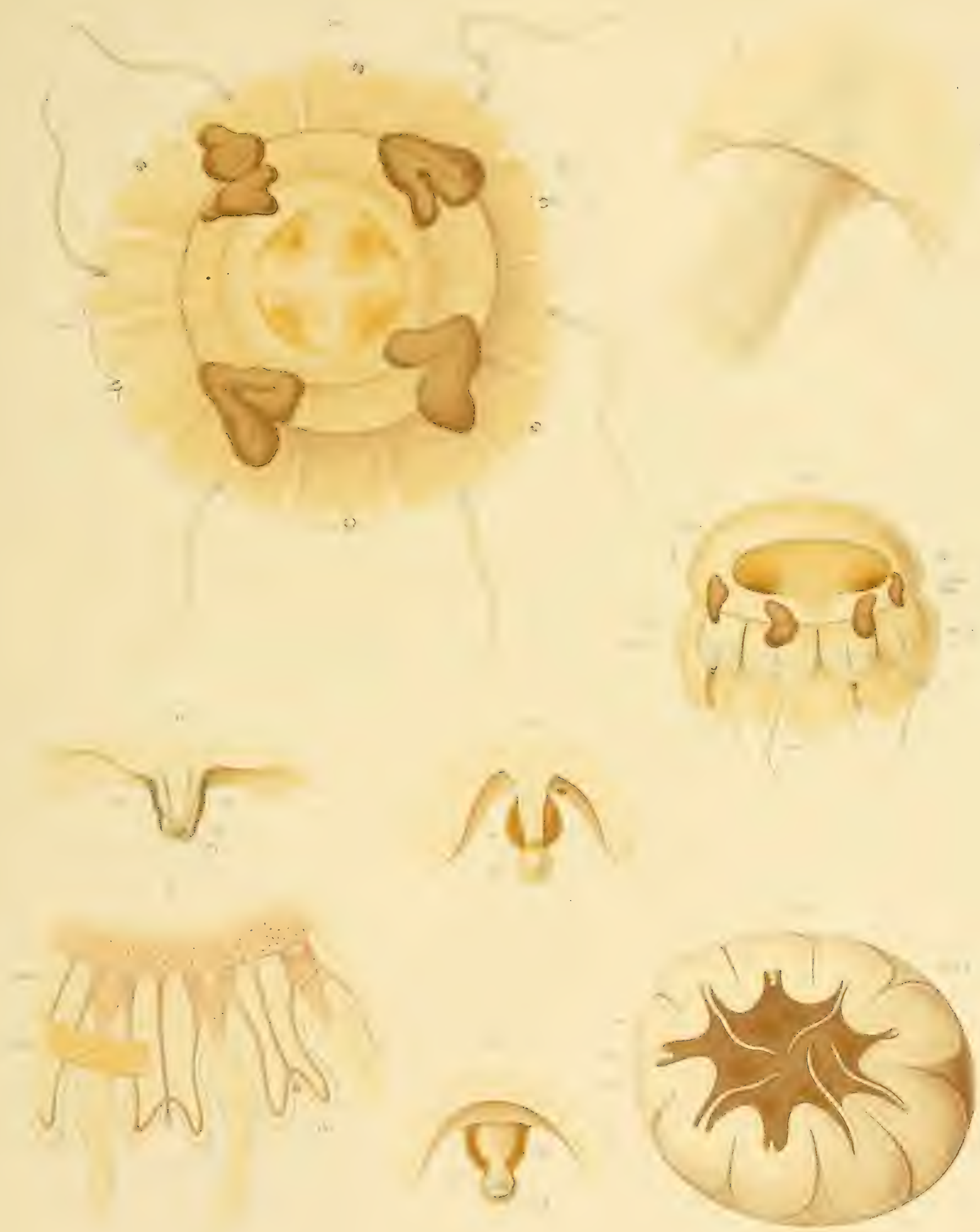



$$
\text { . }
$$




\section{TAFEL IV.}

Sämmtliche Figuren beziehen sich auf Cassiopicia, und zwar Fig. 26, 35, 36 auf C. ornata $n$. var. digitata, die übrigen auf $C$. andromeda Esch. $n$. var. malayensis.

I $\because$ g. 24. Wirklicher Schnitt im Perradius durch die ganze Meduse; zeigt den Zusammenhang der Arme mit der Armscheibe; dagegen ist von den Subgenitalhöhlen nichts zu sehen; nur die Gallerte zeigt (gal. per) besondere Structur. Die Filamente sind auch noch in diesem Radius angeschnitten (fil), ebenso eine Anzahl secundärer Fiederästchen.

Fig. 25. Halber Schnitt durch den Interradius. Die Verbindung von Arm und Armscheibe ist auf diesem Schnitt unterbrochen, dagegen fallen die Subgenitalhöhlen (cav gen) und die Ostien (ost gen) in die Schnittrichtung, ebenso wieder die peripheren Armzweige.

Fig. 26. Armverzweigung von C. ornata var. digitata von oben (natuirl. Farbe).

Fig. 27. Armverzweigung von C. C. andromeda var. malayensis von oben und

Fig. 28. von der Seite. Die Krausen schneiden in einer Ebene ab.

Fig. 29. Arm mit Krausen und Kolben von unten (Subumbrellarseite). In der Mitte die Kolbenrosette, (koc), die zum Anheften von Embryonen dient.

Fig. 30. Subumbrellarmuskulatur; Fiederarcaden mit Umbiegungen in äussere und innere Circulärzuige. Radiale Streifen, die rhopalaren durch Färbung ausgezeichnet.

Fig. 3I-34. Schirmrand mit Rhopalien und Lappen in verschiedenen Altersstadien, um die

Vermehrung der Lappen, die zuerst in Canalsystem (ent), dann an dem inneren Lappenteil (lo) hervortritt, zu zeigen; mit r, 2, 3, 5 mehr oder minder vollkommen eingeschnitten Lappen (s. Text p. 41).

Jis. $3 i .36$. Die Vermehrung bei C. ornata var. digitata. Die Entodermausbuchtungen vorauseilend. 


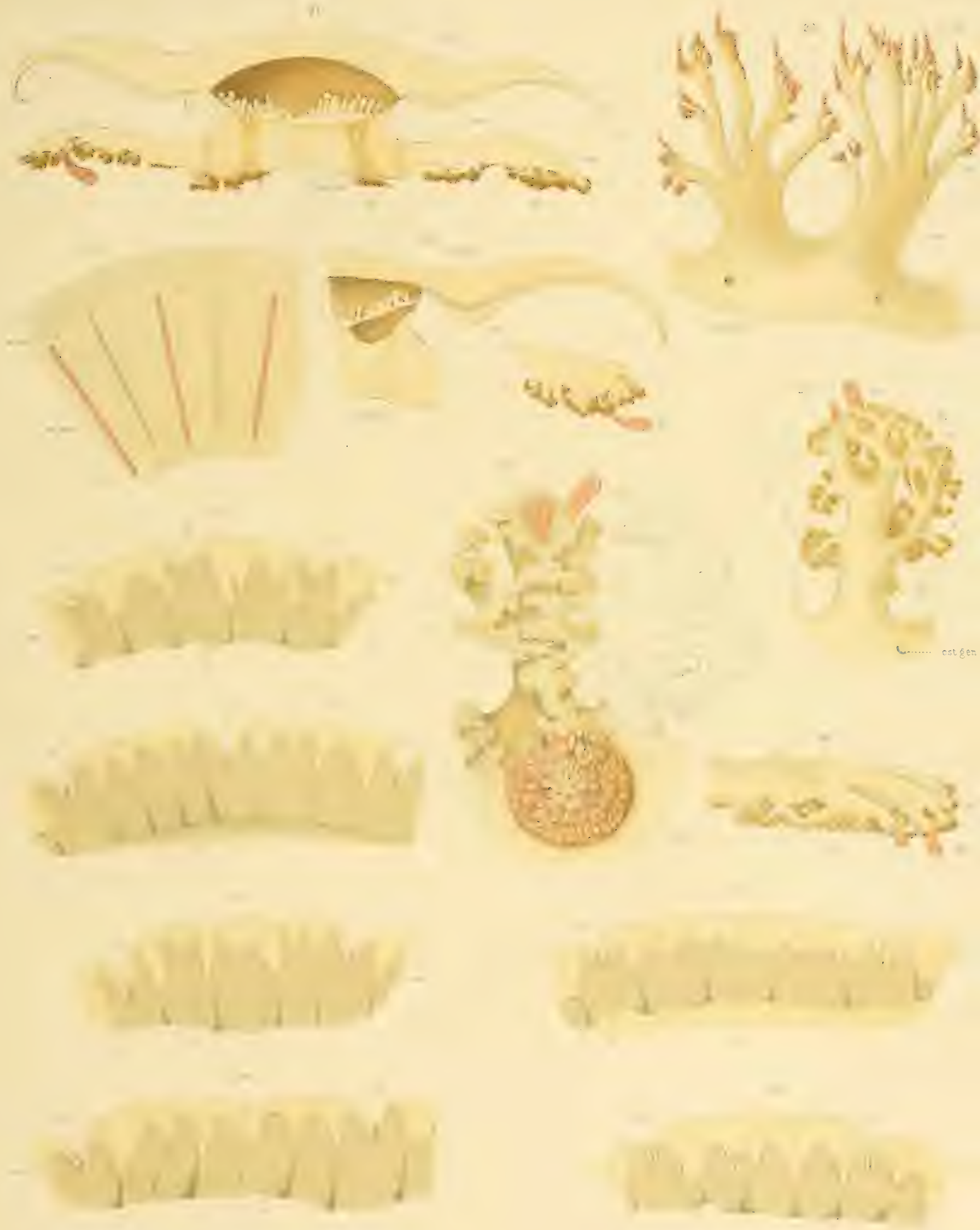






\section{TAFEL V.}

\section{Sämmtliche Figuren von Vetrostoma coerulescons n. sp.}

Iiig. 37. Ein Mundarm von der Seite; man sieht die beiden Hauptflügel $(z)$ und secundäre Fiedern ( $z !)$; ferner die enge Subgenitalöffnung (ost gen).

I.i. 3 . Mundarm von der Aussenseite mit auseinandergepreizten Fliigeln, die secundären Fiederästchen sind wegen ihrer Gedrungenheit nicht zu sehen; die Krausen ( $k r$ ) scheinen direkt den beiden Gabelästen (z) aufzusitzen.

Iin. 39. Nundarmende von oben, die secundären Fiederästchen (z!z!!) durch Pressen sichtbarer gemacht, so dass die fortgesetzte Dichotomie hervortritt.

Firg. fo. Mundarmende (dasselbe Stück) von unten; nur die Hauptgabelung und die Krausen sind sichtbar.

Iing. +1, +2. Exumbrellarkuppel eines grösseren Exemplars von der Seite und von oben gesehen, mit den lappigen Gallertwarzen.

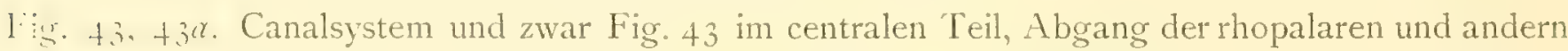
Radiärcanäle von der Nagenperipherie und Beginn der Anastomosen und 4za. Verzweigung in der Subumbrella (genau vom Object durch Glas ibertragen): allmähliche Verengerung des Netzes nach der Peripherie, kein Ringcanal (s. auch Fig. Io9 auf Taf. XII).

I. 4 . Die kleinen Saugkolben an den Armen, stark vergrössert; mit verengertem Halsteil und zahlreichen Nesselwarzen.

I: 4. 4. Die Anhänge der Centralscheibe, Saugkrausen und federartige Gebilde; ebenfalls distal offen, mit zerstreuten Nesselwarzen (nw) und starker Gallerte; einzelne Embryonenballen (ov) anhängend.

Fis. $f^{\prime}$. Habitusbild eines Teils der Subumbrella; die Unterarme sind der starken Umbiegung wegen (s. Fig. 37) nicht sichtbar. Schirmrand etwas eingeschlagen mit den unregelmässigen, wenig eingeschnittenen nur an den Rhopalien deutlicheren Randlappen (s, auch Fig. Iog auf Taf. XII). Radiärmuskulatur (mu. ra.) 

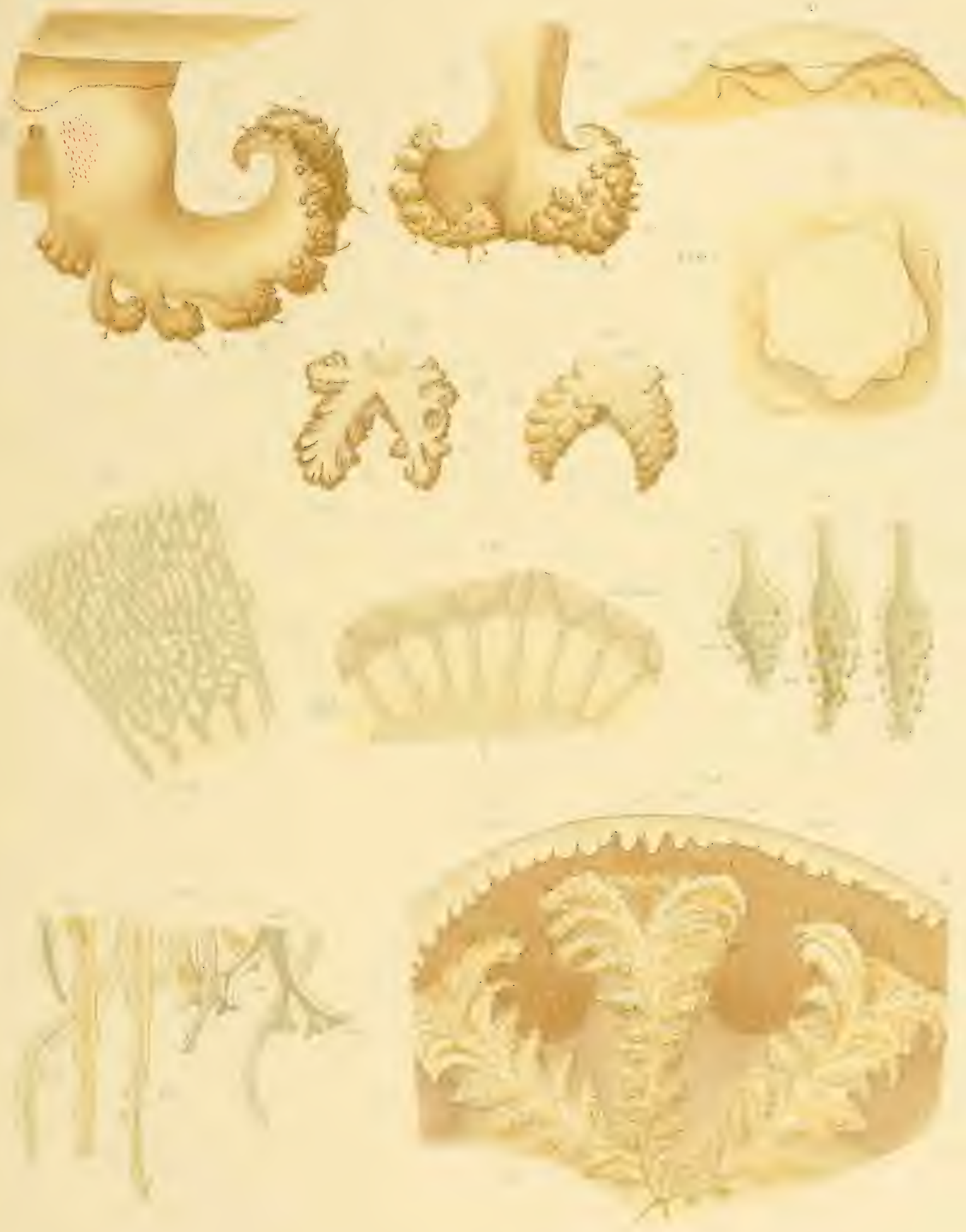




\section{'I'AFEL, VI.}

Fig. 47-53 incl. von Crambione mastigophora n. g. n. sp. Fig. 54-57. Schirmrandstadien von Mastigias papua var. sibogae.

Fig. 4\% Habitus des ganzen Tiers ohne Umbrella nach einem grossen Exemplar ungefähr 4 mal verkleinert. Oberhalb der Armbasen sind die schlitzförmigen interradialen Subgenitalöffnungen zu sehen (ost gen), dazwischen perradial eine Furche, (fos per), die äusserlich ähnlich, jedoch nur seicht ist und auch höher liegt (s. auch Fig. 5o). Zwischen den Armbasen treten die Fiederäste der axialen Krausenkante mit ihren Filamenten hervor. An den Unterarmen legen sich von aussen gesehen die beiden abaxialen Krausenkanten so dicht aneinander, dass nur ein Schlitz (x) sichtbar bleibt. (Vgl. auch Fig. 7r-74, Taf. VIII).

lic. … Canalsystem der Subumbrella von ringförmiger Muskulatur ( $m$ cir) teilweise überdeckt. Innerhalb des Ringcanals ein kleineres, nur mit diesern zusammenhängendes Anastomosennetz, ausserhalb ein enges Netz, in welchem nur die 8 rhopalaren Canäle hervortreten, die 8 adradialen hören am Ringcanal auf. Besondere unverzweigte Canäle in den Rhopalarlappen.

Fig, $f(;$. Abgang der Radialcanäle von der Magenbasis.

Fig. 51. Stick der Armscheibe; die Mundarme an der gezackten Linie abgeschnitten; zeigt die schlitzförmigen, etwas durch Klappen verengten Subgenitalostien (ost gen) und die seichten perradialen Gruben in der Armscheiben-Gallerte (fos per); das Canalsystem durchschimmerend.

Ii: Ki. 5l. Kleine gestielte Kolben der Arme, stark vergr. mit geschlossenem Entoderm und zahlireichen Nesselwarzen.

Iis. 52. Querschnitte durch einen Nundarm, a) durch den oberen Teil mit den seitlichen Fiederästchen, b) durch den unteren Teil mit den beiden abaxialen Flügeln (z), c) durch das untere Ende, wo scheinbar ein allseitiger Krausenbesatz vorhanden ist.

ï... 53. Schirmrand mit den Randlappen zwischen zwei Rhopalien; die einen Lappen tiefer eingeschnitten, höher heraufreichend als andere; an den Rhopalien die muschelförmige äussere Sinnesgrube (fos ol) mit Radiärfalten.

1..... - ; ;. Schirmand von Mastigias papua var. sibogae in verschiedenen Altersstadien. Fig. 5.4. 2 Lappen, jecler wieder zweigeteilt, Fig. 55. 4 Hauptlappen, jeder mit besinnender Zweiteilung, Fig. 56. 6 Hauptlappen, (in zweien des 4 teiligen Stadiums ist die Teilung durchgefiihrt, in 2 noch gar nicht angefangen. Fig. 57 . \& Lappen, aber noch nicht alle vollständig eingeschnitten. 

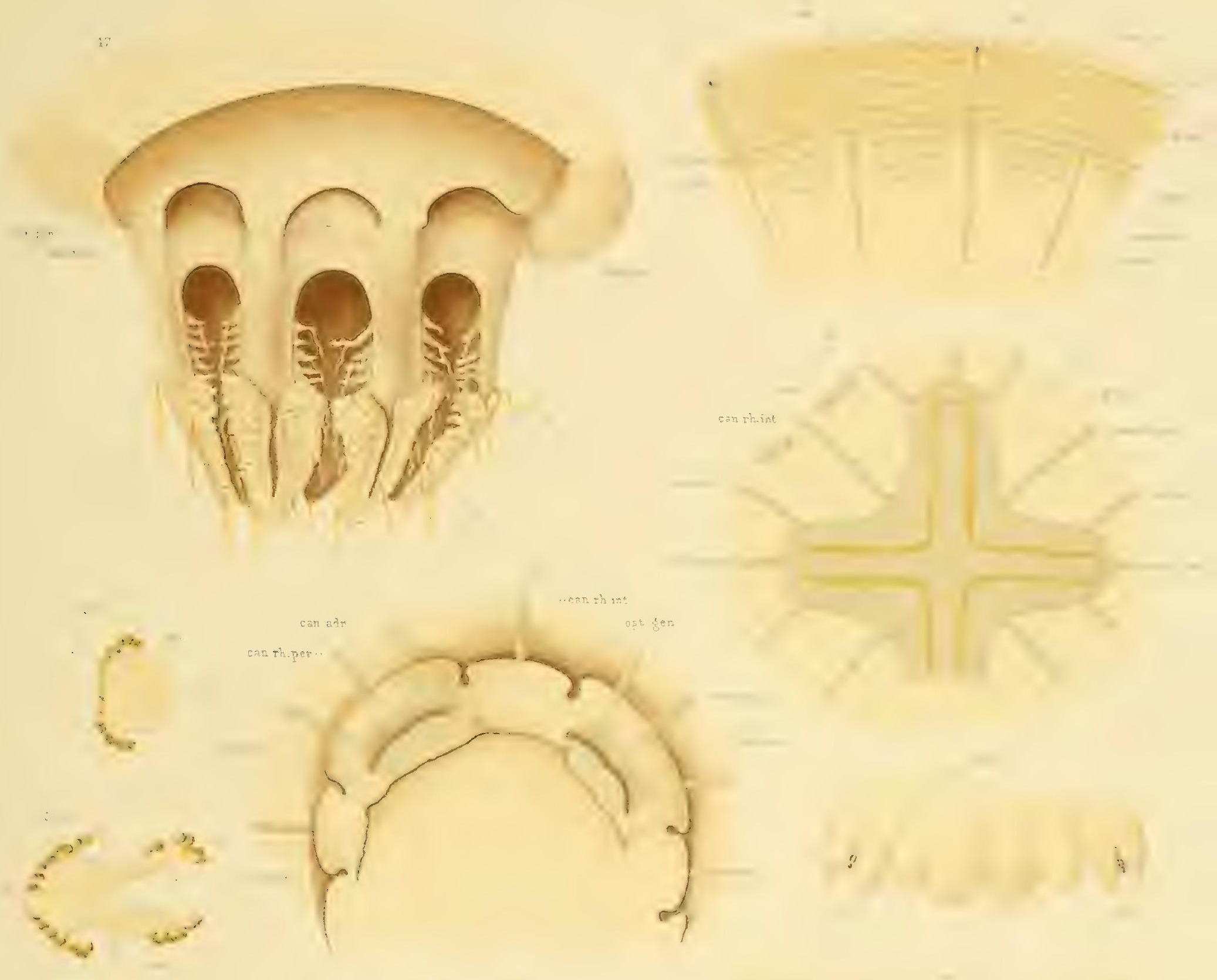

$$
\frac{1}{6+3}
$$

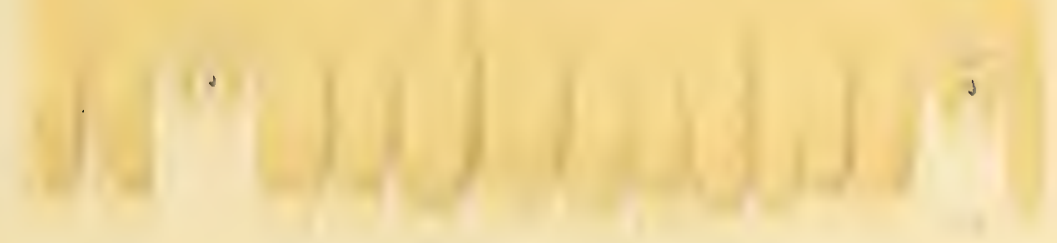






\section{TAFEL VII.}

Fig. 58-64 (incl.) von Mastigias, Fig. 65-68 incl. von Crossostoma.

Fig. $58 . \quad$ Armscheibe, Nagenperipherie und Canalsystem der Subumbrella ohne Muskulatur von Mastigias papua n. var. sibogae. Des Entoderm rosa, in den rhopalaren Radien tiefrot. Anastomosennetz am Magen beginnend; jenseits des Ringcanals plötzlich verengert, distal den Lappen folgend. Die geschweifte Form der perradialen Armbasen bedingt eine entsprechende Ausbuchtung der weiten interradialen Subgenitalostien, in denen die Gonaden heraustreten.

Fig. , (1). Radialcanäle einer sehr jungen Mastigias papua n. var. sibogae mit gestrecktem Verlauf, nicht anastomisirend.

Fig. (x). Radialcanäle einer verwandten Species, ebenfalls junges Exemplar; Anastomosennetz schon fast am Magen beginnend.

I:is. 61. Etwas älteres Exemplar von $M$. papua, var. sibogac; Radiärcanäle beim Abgang noch fast parallel und unverzweigt.

Iiig. 62, 63. Mundarmende und kurze Terminalknöpfe der anderen Mastigias-species.

liig. 64. Armende eines ebenso kleinen Exemplars von 11 . papua var. sibogae mit längeren Kolben.

IVig. 65. Stück der Subumbrella ron Crossostoma anadyomone n. sp. in naturl. Grösse. Die Mundarme an der gezackten Linie von der Armscheibe abgetrennt, um das Canalsystem sichtbar zu machen. Vom eingeengten Magenkreuz gehen perradial 4 Rhopalarcanäle ab; interradial ein weitmaschiges Anastomosennetz, in welchem erst in einiger Entfernung von der Magenperipherie der interradiale Rhopalarcanal hervortritt. Das Canalsystem ist im peripheren Teil durch Muskulatur verdeckt und tritt erst in den Randlappen wieder hervor.

Fig. 66. Ein Mundarm von Crossostoma anadyomene n. sp. mit den seitlichen Lappen (z!), abaxialen Kolben (ko) und den axialen Filamenten (pf) Das doppelte Canalsystem rötlich durchschimmerncl.

I.i. 67. Stick eines Endarms, um die fur jeden Zweig do p pelt te Canalversorgung zu zeigen.

I.is. 68. Centrum der Subumbrella, wo die 8, von den Armen kommenden Krausenkanten in einer Rosette, durch allseitig angesetzte Krausenästchen (kr. c.) gebildet, endigen, untermischt mit Filamenten pf c.); in der seitlichen Ansicht auch in Fig. 68 sichtbar (kr. c. und pf.c.). 

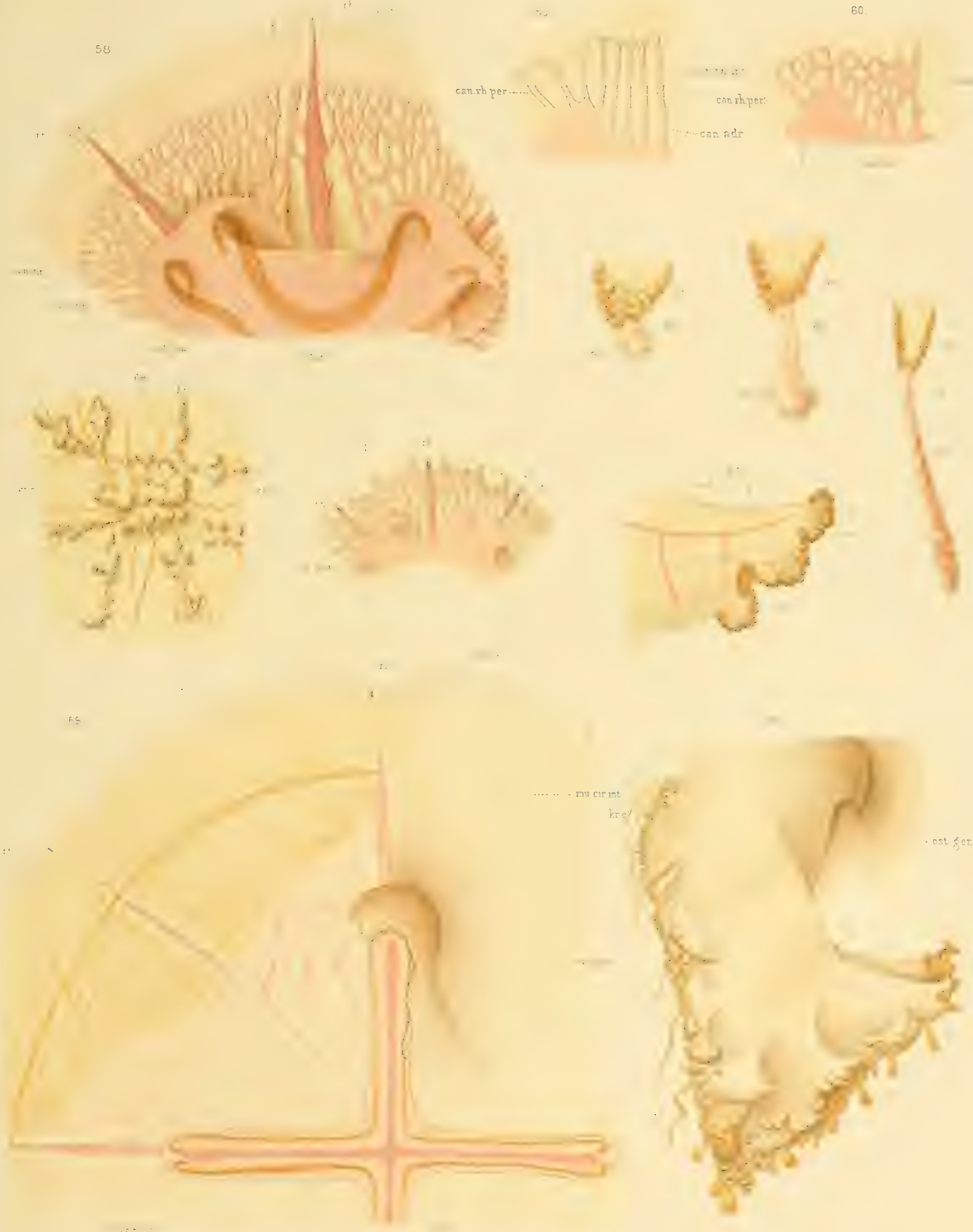




\title{
TAFEL TIII.
}

\begin{abstract}
Armformen verschiedener Rhizostomen zur Ableitung der tripteren (dreiflügligen) von der dichotomen (zweigabligen) Form.
\end{abstract}

Fig. 69, 70. Die dichotome Cotylorhiza tuberculata des Mittelmeers, zum Vergleich herangezogen, Fig. 69 von der Seite mit zusammengelegten, Fig. 70 von aussen mit auseinander gespreizten Flügeln [Fig. 95 auf Taf. X dasselbe mit in natuirlicher Lage zusammengelegten Flugeln, Fig. 86 auf Taf. IX von der axialen Seite aus]. Man vergl. mit Fig. 69 auch die einfache triptere Form der Crossostoma (Fig. 66 auf Taf. VII).

Fig. $7 \mathrm{I}-74$ incl. Mundarm von Crambione mastigophora n. g. n. sp. junges Exemplar. einfache triptere Form. Fig. 7 I von der Seite (vgl. Fig. 69, 66), Fig. 72 von aussen mit gespreizten, Fig. 73 mit naturlich zusammengelegten Flügeln, so dass nur eine Rinne $(\mathrm{x})$ zwischen den abaxialen Krausenreihen bleibt; Fig. 74 von der axialen Seite

Fig. 75-77. Dreifligliger Mundarm mit Endkolben eines jungen Exemplars von Mastigias papua var. sibogae von der Seite (Fig. 75), von aussen mit gespreizten Fligeln (Fig. $76 \mathrm{z}$ ), von innen, axial (Fig. 77). Der Endkolben ebenfalls dreikantig. Hieran schliesst sich zum Vergleich einerseits Fig. 84 (ältere Mastigias) und daran die scapulata (Rhizostoma) Fig. 82 und Rhopilema Fig. 78. Taf. IX, andererseits die lorifera, Himantostoma (Fig. 90) und Thysanostoma (Fig. 93, Taf. X). 

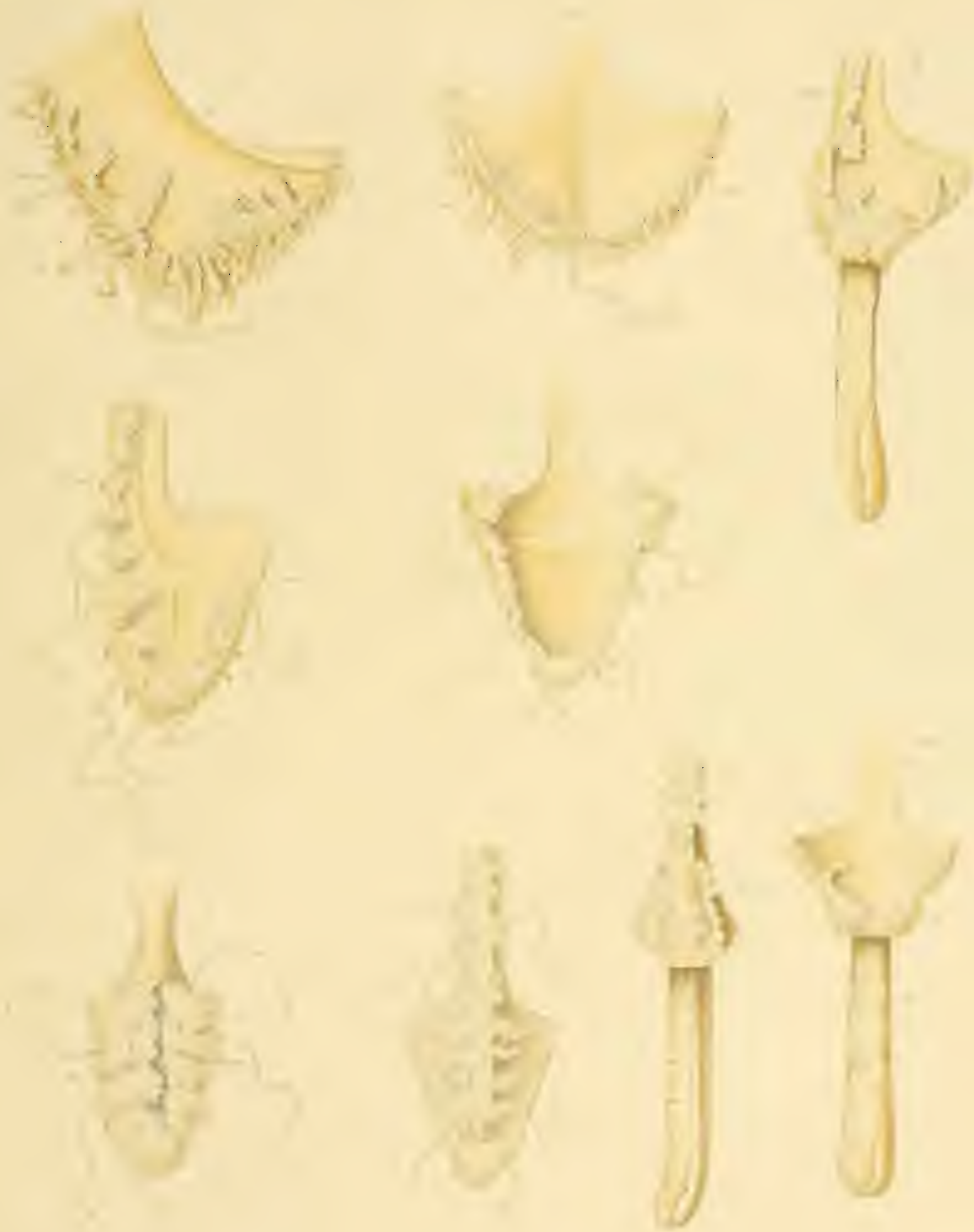

(1) Mara del 



\section{TAFEL IX.}

I.ig. 7\$. Rhopilema hispidum Vanh. Mundarm mit der Schulterkrause (scap.) von der Seite.

Irig. 79. Unterer Teil von aussen mit auseinandergeklappten Flügeln. An den beiden Abaxialflügeln secundäre Spaltung ( $z$ ! und $z ! !)$

lïg. So. Querschnitte durch diesen Unterarm, um das Verhältnis der Hauptflügel (z.), der secundären ( $\mathrm{z}$ !) und der Krausen (kr.) zu zeigen. 80 a im oberen Teil, oberhalb der Linie $\mathrm{x}-\mathrm{y}$ Fig. 78 , 8ob, entspr. $\mathrm{x}_{1}-\mathrm{y}_{1}$.

lïg. S1. Stück der Subumbrella von Rhopiloma hispidum Vanh. mit 2 Rhopalarcanälen (can rh) und einem Zwischencanal (can adr.), den senkrecht abgehenden Anastomosen und den dreieckigen Feldern der Circulärmuskulatur.

Ïig. \$2-\$3. Armbildung einer jungen Rhizostoma pulmo (zum Vergleich herangezogen) von der Seite und von aussen.

Iتig. St-\$5. Armbildung eines älteren Mastigias-exemplars; die Seitenäste der Abaxialflügel (z!) gewirnen grössere Selbstständigkeit, besonders der oberste (z!!) löst sich los. (Uebergang zu 78, 82, 83 Form der scapulata).

Iig. 86. Arm von Cotylorhiza tuberculata von der Axialseite. Die Aehnlichkeit mit dreiflügligen Formen noch durch den an entsprechender Stelle sitzenden grossen Kolben (ko!) verstärkt. 


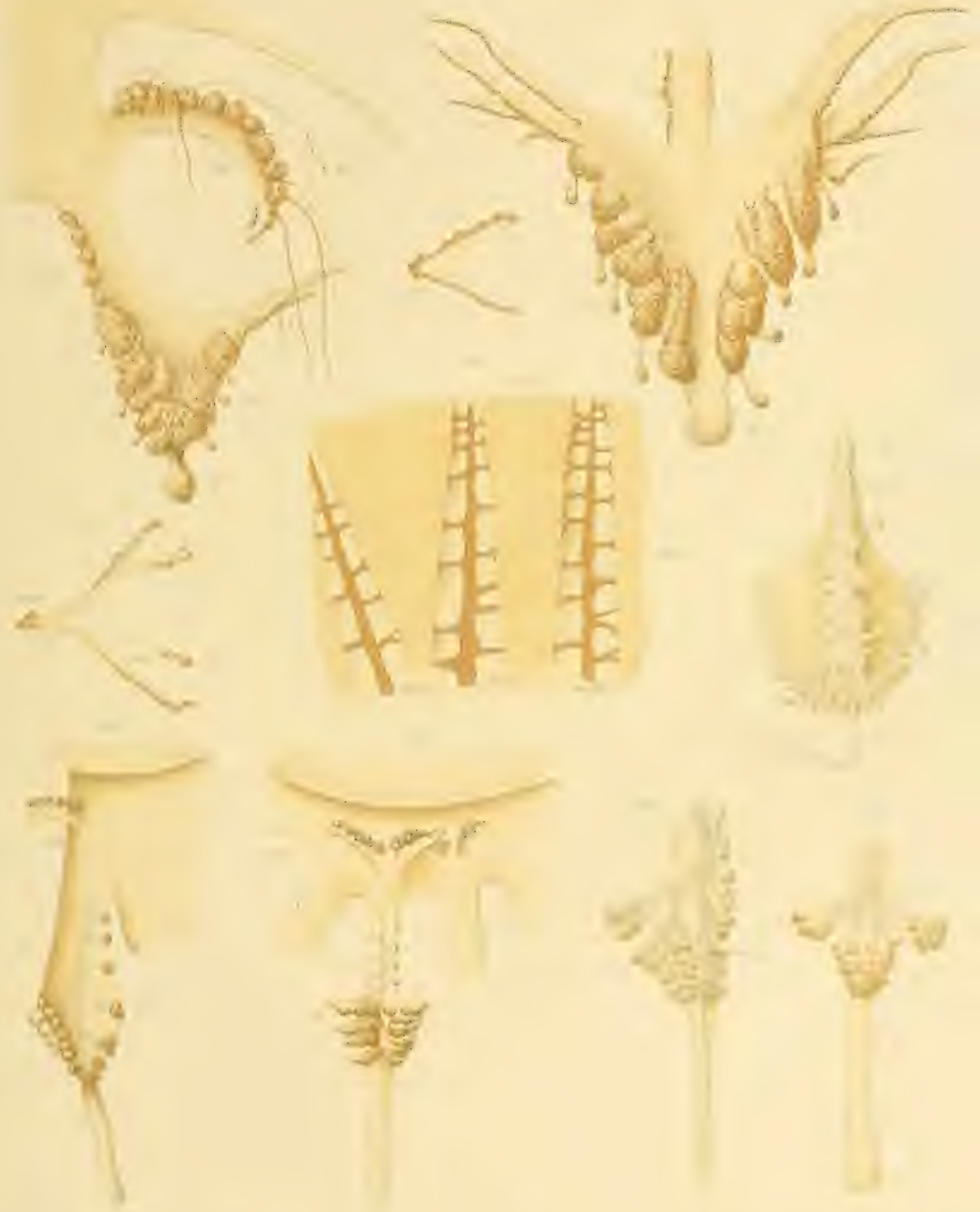






\section{TAFEL X.}

Fig. S6. Himantostoma flagellatum Hckl. Ganze Meduse, verkleinert mit den langen Peitschenfilamenten an den Armkrausen und besonders grossem Anhang am Armende. Durch die teilweise Verwachsung der Oberarme verbleiben zwischen den Oberarmen nur niedrige Fenster (vgl. dagegen Fig. 47, Taf. VI).

Fis. S. Stiuck der Armscheibe von Himantostoma flagellatum Hckl. zeigt das die Armbasen verbindende Patagium (pat).

Fin. Sici. Schirmrand von Himantostoma flagcllatum Hckl. mit den spitzen, rot getupften Randlappen (etwa 8) zwischen 2 Rhopalien.

IFis. (m. u1. Iundarm von Himantostoma flagellatum Hckl. von der Seite und von aussen; Abaxial-Fligel, soweit frei, aufgeklappt; im unteren Teil Querschnitt dreikantig, keine Fluigel erkennbar, Krausen allseitig (vgl. auch Fig. 75, 76 etc.).

1:... 2. Zeigt dies an Querschnitten, 92a durch oberen Teil, Linie $x-y$ Fig. $90 ; 92 b$ durch den unteren Teil, Linie $x_{1}-y_{1}$.

Iت3. 1,3. Mundarm von Thy'sanostoma thysanura Hckl. Im oberen Teil noch mit seitlichen Aestchen (z!) auf den Hauptfligeln; Flügelform noch in der ganzen Länge des Arms erhalten, wie die Querschnitte zeigen.

Fig. 94. $\quad a$ durch den oberen Teil, Linie $x-y$ '.

$b$ durch den unteren Teil, Linie $x_{1}-y_{1}^{\prime}$. 

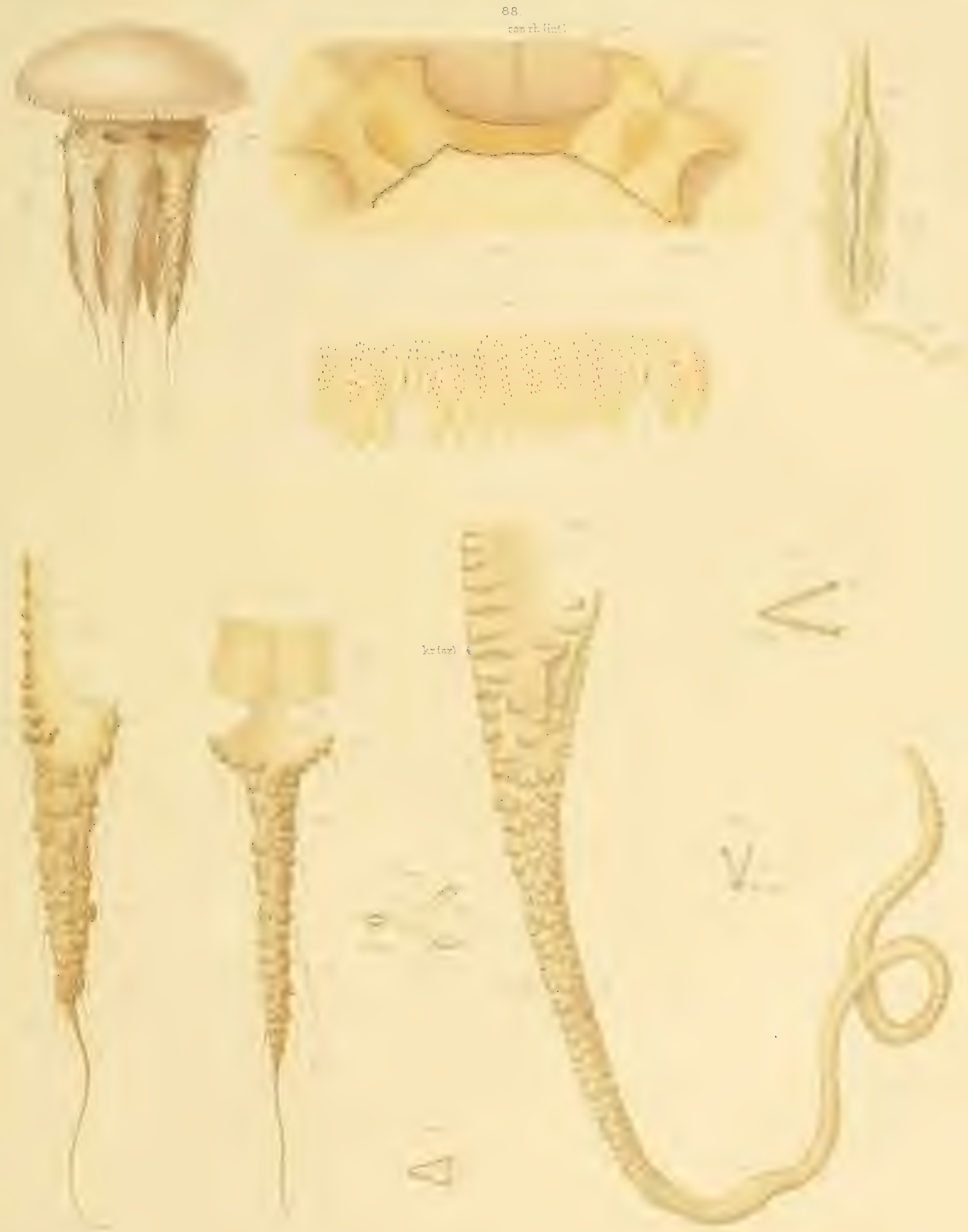

O. Maas, del. 




\section{TAFEL XI.}

Mit Ausnahme von Fig. 96 Rhopalien im Aufsichtsbild und im Schnitt.

I\%ig. 96. Ursprung der Genitalfalte bei Netrostoma cocmulescens n. sp. flach ausgebreitet. Die jüngsten erkennbarer Eier (ov!) liegen zunächst den Gastralfilamenten.

Fig. 97-IOI. Rhopalien von Rhizostomeen im Aufsichtsbild bei schwächerer Vergrösserung (Zeiss A. oc. I) und zwar.

Fig. 97. Von Netrostoma cocmulescens n. sp. ohne Pigmentfleck und ohneäussere Sinnesgrube; nur mit Sinnesepithel (ect!) an Bulbus und Otolithensack, das durch die Deckschuppe hindurch sichtbar ist.

Fig. 98. Cassiopcia andromeda Esch. n. var. malayensis. Kleine äussere Sinnesgrube von Sichelform an der Basis des Rhopaliums auf der Exumbrella. Kleiner Pigmentfleck (oc) am Distalende des Concrementsacks durch die Deckschuppe (sq) schimmernd.

Fig. 99. Mastigias papua var. sibogae mit grosserer äusserer Sinnesgrube und sehr starkem Pigmentfleck.

Fig. 100. Crambione mastigophora n. g. n. sp. Aeussere Sinnesgrube sehr gross muschelförmig (vgl. Fig. 53, Taf. VI) mit radiären Rinnen und Falten. Auf dem Kolben ausser den beiden Sinnesepithelien ein paariger Ocellus an der Basis des eigentlichen Concrementsacks sichtbar.

Fig. IоI. Himantostoma flagcllatım Hckl. Aeussere Sinnesgrube wie oben. Pigmentfleck unpaar Deckschuppe auffallend breit.

Fig. I02-I05 Schnitte von diesen Kolben. Vergr. C. oc. I.

Fig. IO2. Cassiopeia andromeda n. var. malayensis, frontaler Längsschnitt durch das Rhopalium, rechts und links das Sinnesepithel des Bulbus getroffen.

Fig. I03. Netrostoma cocmulescons n. sp. radialer Längsschnitt durch das Rhopalium, entodermaler Canal bis zum Concrementsack durchgängig, nur vor dem Bulbus, der hier sehr schwach ist, verengert. Ausser diesen Teilen nebst ihren Sinnesepithelien keinerlei Differenzierung, weder Auge noch äussere Sinnesgrube.

Fig. IO4. Crambione mastigophora n. g. n. sp. Radialer Längsschnitte neben der Kolbenmitte. Einer der paarigen Ocelli zwischen Bulbusteil und Endteil des Otolithensacks getroffen. Aeussere Sinnesgrube (fos ol) mit tiefen Rinnen und Falten.

Fig. 105. Mastigias papua n. var. sibogae. Der unpaare becherförmige Ocellus zwischen den Entodermzellen des Otolithensacks getroffen. Aeussere Sinnesgrube ohne Falten. Sinnesepithelien (ect!) wie oben.

Fig. 106. Rhopalium von Paraphyllina intermedia n. g. n. sp. (vgl. Fig. r4, Taf. II) im wirklichen radialen Längsschnitt. Zeiss E. oc. I. Endteil mit Concrementen vom bulbösen Teil abgeschnuirt, aber durch Zellen durch die Gallerte hindurch damit in Verbindung. Auf der centralen Seite ein Auge mit Pigmentzellenbecher und darin einem lichtbrechenden Organ (1i). Darunter wie am dorsalen Teil des Otolithensacks ein stark entwickeltes Sinnesepithel (ect!), mit basal liegenden Fasern und Ganglienzellen (nz). Ebenso dorsal und am Bulbus. 


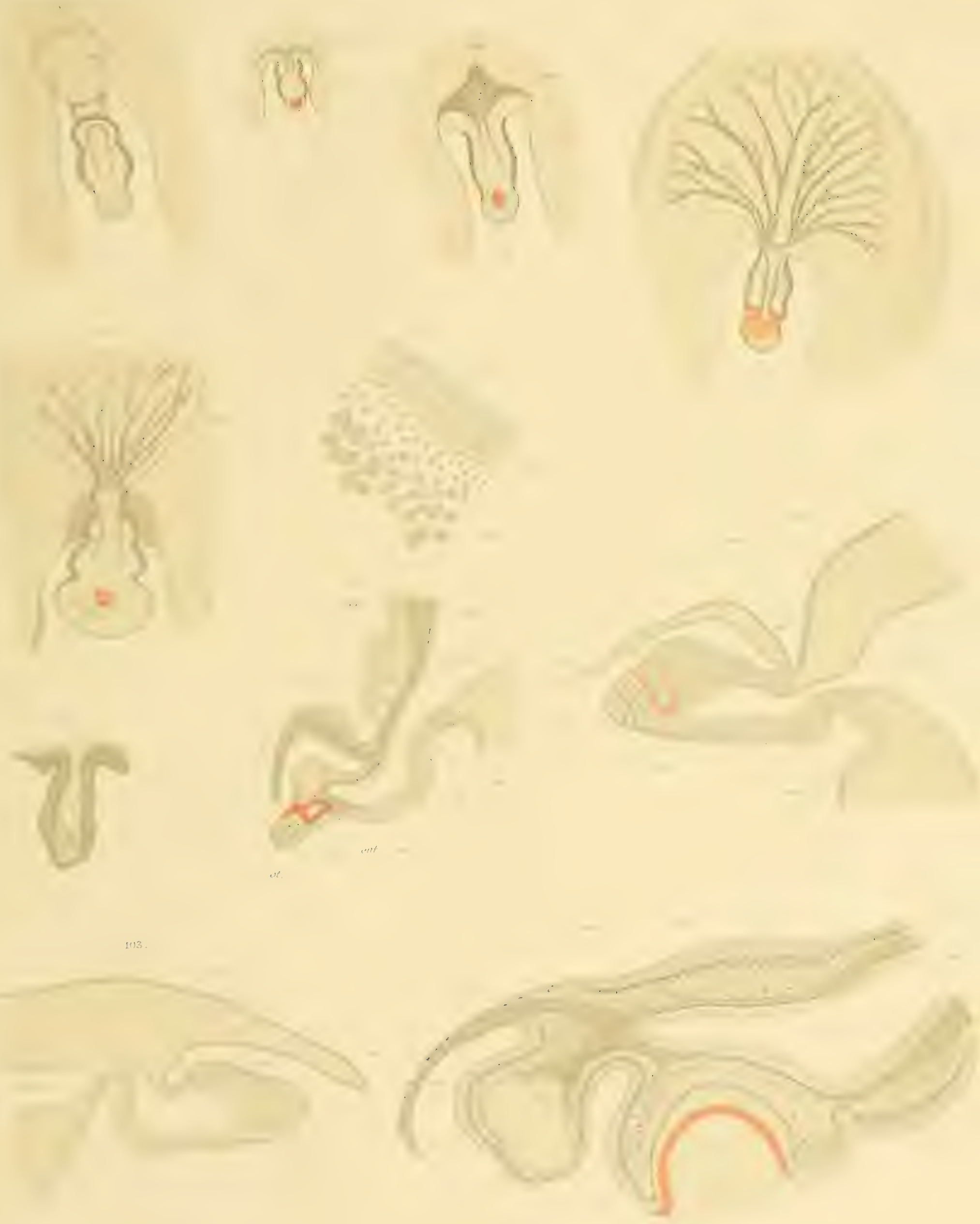






\section{TAFEL XII.}

Habitusbilder nach Photographien.

Fing. 107. Periphylla dodecabostryecha Brdt.

Fins. ios. Atolla Valdiviae Vanh.

Fig. I Iog. Netrostoma coerulescens n. sp.

Fir. 110. Mastigias papua L. Ag. n. var. sibogae.

Fing. I I I. Mastigias spec. B.

Iïg. I I 2. Cassiopeia andromeda Esch n. var. malayensis.

Iing. II . Crambione mastigophora n. g. n. sp. 
Siboga-Expeditie XI O. Maas Scyphomedusae.

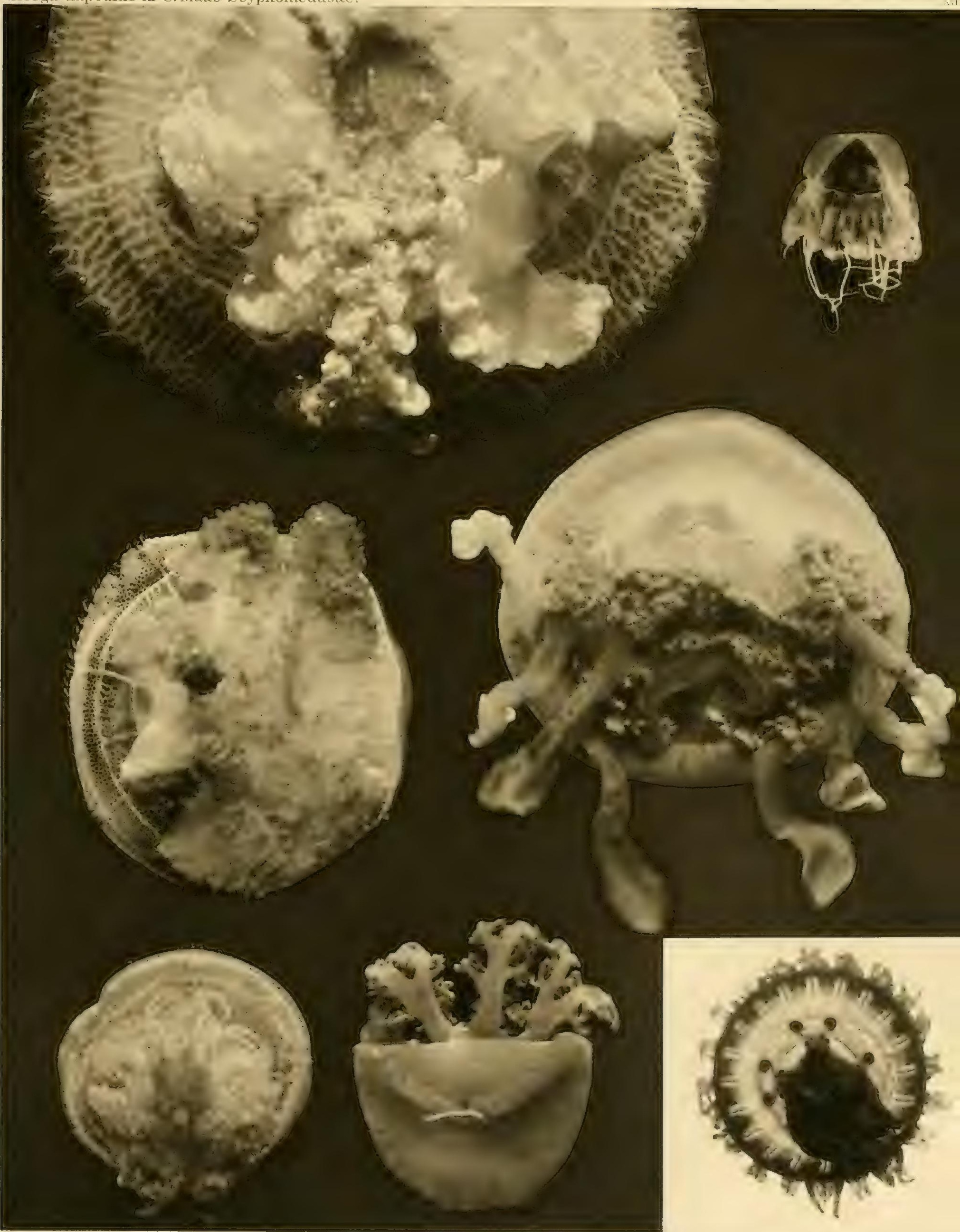






\section{CONDITIONS GÉNÉRALES DE VENTE.}

I․ L'ouvrage du "Siboga" se composera d'une série de monographies.

$2^{\circ}$. Ces monographies paraîtront au fur et à mesure qu'elles seront prêtes.

$3^{\circ}$. Le prix de chaque monographic sera différent, mais nous avons adopté comme base générale du prix de vente: pour une feuille d'impression sans fig. flor. 0.15 ; pour une feuille avec fig. flor. 0.20 à 0.25 ; pour une planche noire flor. 0.25 ; pour une planche coloriée flor. 0.40 .

$4^{\circ}$. Il y aura deux modes de souscription:

a. La souscription à l'ouvrage complet.

b. La souscription à des monographies séparées en nombre restreint.

Dans ce dernier cas, le prix des monographies sera majoré de $25 \%$.

$5^{\circ}$. L'ouvrage sera réuni en volumes avec titres et index. Les souscripteurs à l'ouvrage complet recevront ces titres et index, au fur et à mesure que chaque volume sera complet.

\section{Déjà paru:}

Ie Livraison. (Monographie XLIV) C. Ph. Sluiter. Die Holothurièn der Siboga-Expedition. Mit Io Taf. Pour les souscripteurs à l'ouvrage complet. $f 7.50$ $2^{e}$ Livraison. (Monographie LX) E. S. Barton. The genus Halimeda. With 4 plates. . . . $f$ f.40 Pour les souscripteurs à l'ouvrage complet.

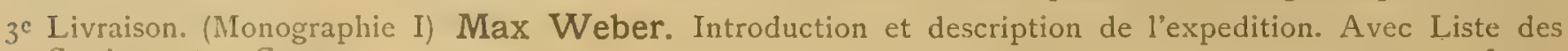
Stations et 2 Cartes. . . . . . . . . . . . f $f$. . Pour les souscripteurs à l'ouvrage complet. $\quad 6.75$

$4^{e}$ Livraison. (Monographie II) G. F. Tydeman. Description of the ship and appliances used for scientific exploration. With 3 plates and illustrations............... $f 2.50$

Pour les souscripteurs à l'ouvrage complet. on 2.

$5^{c}$ Lirraison. (Monographic XIVII) H. F. Nierstrasz. The Solenogastres of the Siboga-Expedition. With six plates

Pour les souscripteurs à l'ourvage complet $f 4.90$

6e Livraison. (Monographic XIII) J. Versluys. Die Gorgoniden der Siboga-Expedition.

I. Dic Chrysogorgiidae. . . . . . . . . f 3.75

Pour les souscriptenirs à l'ouvrage complet. $\quad 3.75$

$7^{*}$ Livraison. (MIonographie XVIa) A. Alcock. Report on the Deep-Sea Madreporaria of the SibogaExpedition. With five plates. . . . . . . . . . . . . . $f 5.75$

Pour les souscripteurs à l'ouvrage complet. 4.60

Se Livraison. (Monographie XXV) C. Ph. Sluiter. Die Sipunculiden und Echiuriden der Siboga-Expedition. Nit vier Tafeln und drei Figuren im Text............. . . f 3.75

Pour les souscriptenrs à l'ouvrage complet. in $3 .--$

ge Livaison. (Mfomographic xi) G. C. J. Vosmaer and J. H. Vernhout. The Porifera of the SibogaExpedition. With five plates. . . . . . . . . . . . f $3^{-}$

Pour les souscripteurs ic louvrage complet. $\quad 2.40$ 




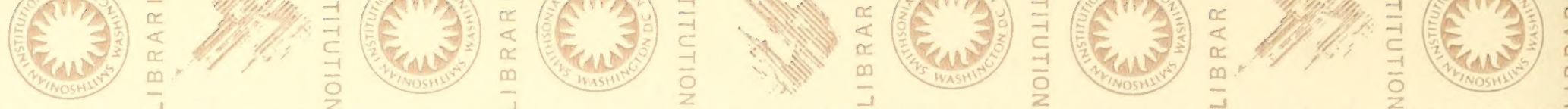

(1)

of

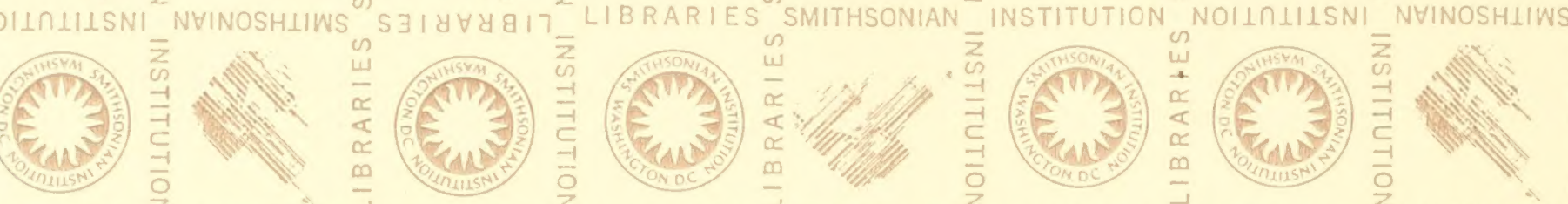

E)

Wive

(iving

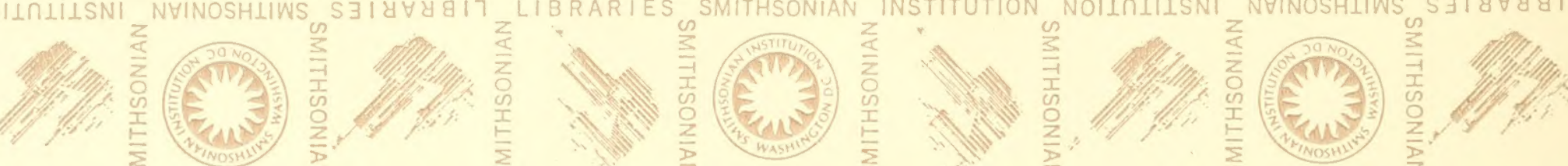

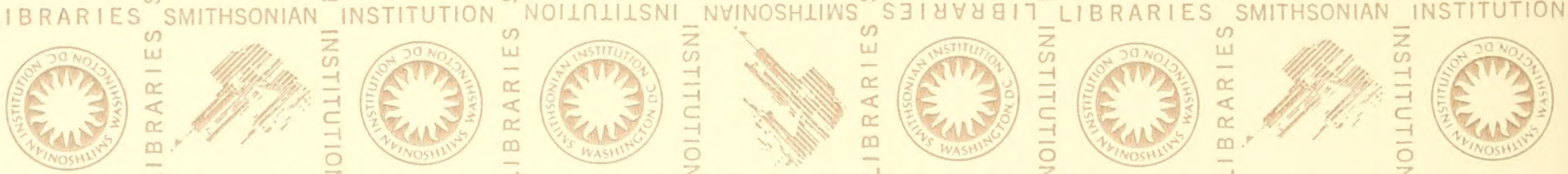

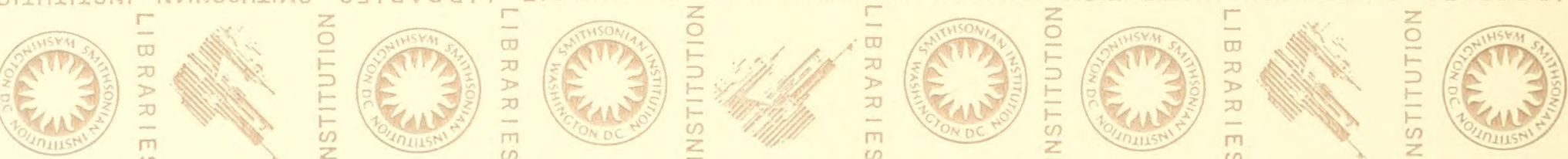

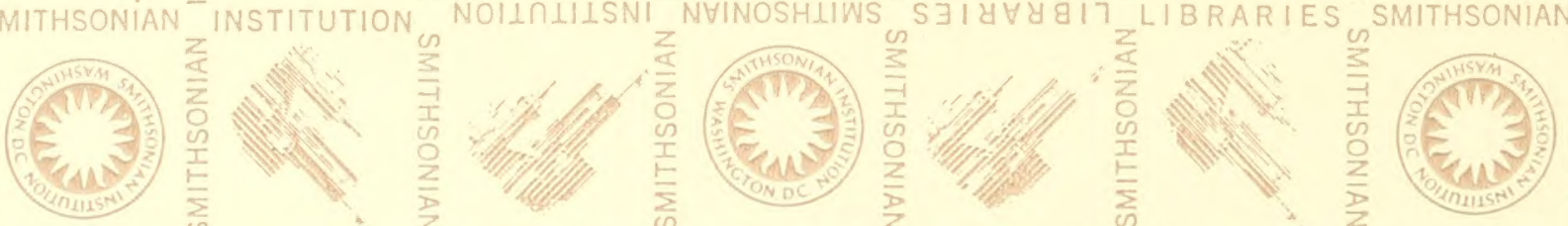

(S)

(N)

VIIII)
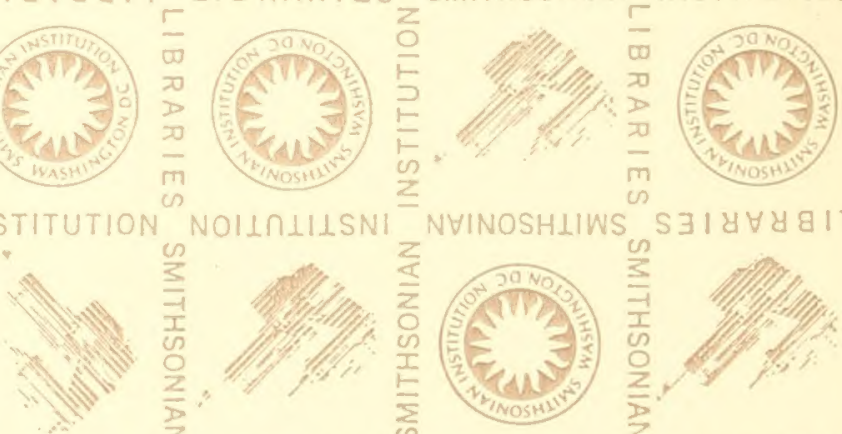

5 S

(S)

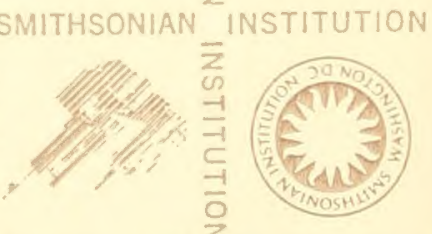

N INSTITUTION NOILIILSNI NHINOSHLINS ${ }^{2}$ S 31 I 
